\title{
THE FIRST HUNDRED BROWN DWARFS DISCOVERED BY THE WIDE-FIELD INFRARED SURVEY EXPLORER (WISE)
}

\author{
J. Davy Kirkpatrick ${ }^{1}$, Michael C. Cushing ${ }^{2}$, Christopher R. Gelino ${ }^{1}$, Roger L. Griffith ${ }^{1}$, Michael F. Skrutskie ${ }^{3}$, \\ Kenneth A. Marsh ${ }^{1}$, Edward L. Wright ${ }^{4}$, A. Mainzer ${ }^{2}$, Peter R. Eisenhardt ${ }^{2}$, Ian S. McLean ${ }^{4}$, Maggie A. Thompson $^{5}$, \\ James M. Bauer ${ }^{2}$, Dominic J. Benford ${ }^{6}$, Carrie R. Bridge ${ }^{7}$, Sean E. Lake ${ }^{4}$, Sara M. Petty ${ }^{4}$, S. A. Stanford ${ }^{8}$, \\ Chao-Wei Tsai ${ }^{1}$, Vanessa Bailey ${ }^{9}$, Charles A. Beichman ${ }^{1}$, Joshua S. Bloom ${ }^{10}$, John J. Bochanski ${ }^{11,12}$, \\ Adam J. Burgasser ${ }^{13}$, Peter L. Capak ${ }^{14}$, Kelle L. Cruz ${ }^{15}$, Philip M. Hinz ${ }^{9}$, Jeyhan S. Kartaltepe ${ }^{16}$, Russell P. Knox ${ }^{9}$, \\ Swarnima Manohar ${ }^{17}$, Daniel Masters ${ }^{18}$, Maria Morales-Calderón ${ }^{14}$, Lisa A. Prato ${ }^{19}$, Timothy J. Rodigas ${ }^{9}$, \\ Mara Salvato $^{20}$, Steven D. Schurr ${ }^{21}$, Nicholas Z. Scoville ${ }^{17}$, Robert A. Simcoe ${ }^{11}$, Karl R. Stapelfeldt ${ }^{2}$, \\ Daniel Stern ${ }^{2}$, Nathan D. Stock ${ }^{9}$, and William D. VaCCa ${ }^{22}$ \\ ${ }^{1}$ Infrared Processing and Analysis Center, MS 100-22, California Institute of Technology, Pasadena, CA 91125, USA; davy@ipac.caltech.edu \\ 2 NASA Jet Propulsion Laboratory, California Institute of Technology, Pasadena, CA 91109, USA \\ ${ }^{3}$ Department of Astronomy, University of Virginia, Charlottesville, VA 22904, USA \\ ${ }^{4}$ Department of Physics and Astronomy, UCLA, Los Angeles, CA 90095-1547, USA \\ ${ }^{5}$ The Potomac School, 1301 Potomac School Road, McLean, VA 22101, USA \\ ${ }^{6}$ Infrared Astrophysics Branch, NASA-Goddard Space Flight Center, 8800 Greenbelt Road, Greenbelt, MD 20771, USA \\ ${ }^{7}$ Division of Physics, Mathematics, and Astronomy, MS 220-6, California Institute of Technology, Pasadena, CA 91125, USA \\ ${ }^{8}$ Department of Physics, University of California, Davis, CA 95616, USA \\ ${ }^{9}$ Steward Observatory, The University of Arizona, 933 North Cherry Avenue, Tucson, AZ 85721, USA \\ ${ }^{10}$ Department of Astronomy, University of California, Berkeley, CA 94720-3411, USA \\ ${ }^{11}$ Massachusetts Institute of Technology, 77 Massachusetts Avenue, Building 37, Cambridge, MA 02139, USA \\ 12 Astronomy and Astrophysics Department, Pennsylvania State University, 525 Davey Laboratory, University Park, PA 16802, USA \\ ${ }_{13}$ Department of Physics, University of California, San Diego, CA 92093, USA \\ ${ }^{14}$ Spitzer Science Center, California Institute of Technology, Pasadena, CA 91125, USA \\ 15 Department of Physics and Astronomy, Hunter College, New York, NY 10065, USA \\ ${ }^{16}$ National Optical Astronomy Observatory, 950 North Cherry Avenue, Tucson, AZ 85719, USA \\ ${ }^{17}$ California Institute of Technology, MC 104-24, Pasadena, CA 91125, USA \\ ${ }^{18}$ Department of Physics and Astronomy, University of California, Riverside, CA, 92521, USA \\ ${ }^{19}$ Lowell Observatory, 1400 West Mars Hill Road, Flagstaff, AZ 86001, USA \\ ${ }^{20}$ Max-Planck-Institut für Plasmaphysik, Boltzmanstrasse 2, D-85741 Garching, Germany \\ ${ }^{21}$ Planck Science Center, MS 220-6, California Institute of Technology, Pasadena, CA 91125, USA \\ ${ }^{22}$ SOFIA-USRA, NASA Ames Research Center, Moffett Field, CA 94035, USA \\ Received 2011 May 31; accepted 2011 August 22; published 2011 November 22
}

\begin{abstract}
We present ground-based spectroscopic verification of $6 \mathrm{Y}$ dwarfs (see also Cushing et al.), $89 \mathrm{~T}$ dwarfs, $8 \mathrm{~L}$ dwarfs, and $1 \mathrm{M}$ dwarf identified by the Wide-field Infrared Survey Explorer (WISE). Eighty of these are cold brown dwarfs with spectral types $\geqslant \mathrm{T} 6$, six of which have been announced earlier by Mainzer et al. and Burgasser et al. We present color-color and color-type diagrams showing the locus of M, L, T, and Y dwarfs in WISE color space. Near-infrared and, in a few cases, optical spectra are presented for these discoveries. Near-infrared classifications as late as early Y are presented and objects with peculiar spectra are discussed. Using these new discoveries, we are also able to extend the optical T dwarf classification scheme from T8 to T9. After deriving an absolute WISE $4.6 \mu \mathrm{m}$ (W2) magnitude versus spectral type relation, we estimate spectrophotometric distances to our discoveries. We also use available astrometric measurements to provide preliminary trigonometric parallaxes to four of our discoveries, which have types of L9 pec (red), T8, T9, and Y0; all of these lie within 10 pc of the Sun. The Y0 dwarf, WISE $1541-2250$, is the closest at $2.8_{-0.6}^{+1.3} \mathrm{pc}$; if this $2.8 \mathrm{pc}$ value persists after continued monitoring, WISE 1541-2250 will become the seventh closest stellar system to the Sun. Another 10 objects, with types between T6 and $>$ Y0, have spectrophotometric distance estimates also placing them within $10 \mathrm{pc}$. The closest of these, the T6 dwarf WISE $1506+7027$, is believed to fall at a distance of $\sim 4.9 \mathrm{pc}$. WISE multi-epoch positions supplemented with positional info primarily from the Spitzer/Infrared Array Camera allow us to calculate proper motions and tangential velocities for roughly one-half of the new discoveries. This work represents the first step by WISE to complete a full-sky, volume-limited census of late-T and Y dwarfs. Using early results from this census, we present preliminary, lower limits to the space density of these objects and discuss constraints on both the functional form of the mass function and the low-mass limit of star formation.
\end{abstract}

Key words: brown dwarfs - infrared: stars - parallaxes - proper motions - solar neighborhood - stars: luminosity function, mass function

Online-only material: color figures, extended figure, machine-readable tables

\section{INTRODUCTION}

Brown dwarfs, objects whose central temperatures never reach the critical threshold for stable thermonuclear burning
(Kumar 1963; Hayashi \& Nakano 1963), are the lowest mass products of star formation. Hundreds of examples are now known, ${ }^{23}$ enabling the study of brown dwarfs as a population in

\footnotetext{
${ }^{23}$ See http://DwarfArchives.org.
} 
their own right (Kirkpatrick 2005). The study of brown dwarfs helps to constrain mechanisms for small-object formation, which include turbulent fragmentation (Padoan et al. 2005; Boyd \& Whitworth 2005), magnetic field confinement (Boss 2004), stellar embryo ejection through dynamical interactions (Reipurth \& Clarke 2001; Bate \& Bonnell 2005), and photoevaporation of embryos by nearby hot stars (Whitworth \& Zinnecker 2004).

Brown dwarfs also represent a "fossilized" record of star formation throughout the Galaxy's history because their mass is never ejected back into the interstellar medium. They therefore preserve information on metallicity enrichment over the lifetime of the Milky Way (Burgasser 2008). Solitary brown dwarfs have also proven to be excellent calibrators of the atmospheric models on which our inference of the properties of giant exoplanets depends (Fortney et al. 2005; Barman et al. 2005; Marois et al. 2008). Their effective temperatures are similar to those of the exoplanets discovered thus far but their spectra lack the complication of irradiation from a host star.

Despite uncovering hundreds of brown dwarfs, previous surveys have allowed us to identify only the warmest examples. The latest object currently having a measured spectrum is UGPS J072227.51-054031.2, whose effective temperature is estimated to be $520 \pm 40 \mathrm{~K}$ (Lucas et al. 2010; Bochanski et al. 2011, find $T_{\text {eff }}=500-600 \mathrm{~K}$ ) and whose spectrum is used as the near-infrared T9 spectral standard (Cushing et al. 2011). ${ }^{24}$ Two other objects-WD 0806-661B (also known as GJ 3483B; Luhman et al. 2011) and CFBDSIR J145829+101343B (Liu et al. 2011) —are probably even colder and later in type than UGPS J072227.51-054031.2, although both currently lack spectroscopic confirmation. Both of these objects underscore the fact that the coldest brown dwarfs are extremely faint even at near-infrared wavelengths where groundbased spectroscopy has its best chance of characterizing the spectra. WD 0806-661B, a common proper-motion companion to a white dwarf with a measured distance of $19.2 \pm 0.6 \mathrm{pc}$, has yet to be detected in ground-based imaging observations down to $J=23.9$ mag (Luhman et al. 2011; see also Rodriguez et al. 2011). CFBDSIR J145829+101343B is the secondary in a system with a composite spectral type of $\mathrm{T} 9$ and a measured distance of $23.1 \pm 2.4 \mathrm{pc}$ (Liu et al. 2011). A combination of its close proximity $(0.11 \mathrm{arcsec})$ to the primary along with a faint magnitude $(J=21.66 \pm 0.34)$ make the acquisition of a spectrum challenging. Of the two, WD 0806-661B is less luminous at the $J$ band and presumably intrinsically fainter bolometrically (see also Wright et al. 2011). Finding even closer and brighter examples of cold brown dwarfs will be necessary to maximize our chances of best characterizing them.

Canvassing the immediate solar neighborhood for such cold objects is one of the goals of the all-sky mission performed by the Wide-field Infrared Survey Explorer (WISE; Wright et al. 2010). Discoveries will directly measure the low-mass cutoff of star formation (Figure 12 of Burgasser 2004) and provide even colder fiducial atmospheres for modeling cold exoplanets and understanding the gas giants of our own solar system. The discovery of cold objects raises the question of whether a new spectral class, dubbed "Y" (Kirkpatrick 2000, 2008; see also Kirkpatrick et al. 1999), will be needed beyond the T class. In this paper, we present an overview of the our first $\sim 100$ WISE brown dwarf discoveries and show that objects colder

\footnotetext{
24 Previously published objects with spectral types $\geqslant \mathrm{T} 8.5$ have been reclassified now that the end of the T dwarf sequence and beginning of the $\mathrm{Y}$ dwarf sequence has been defined (Cushing et al. 2011).
}

than those previously known, including Y-class brown dwarfs, are being uncovered.

\section{BROWN DWARF SELECTION}

WISE is an Earth-orbiting NASA mission that surveyed the entire sky simultaneously at wavelengths of 3.4, 4.6, 12, and $22 \mu \mathrm{m}$, hereafter referred to as bands $W 1, W 2, W 3$, and $W 4$, respectively. As shown in Figures 6, 7, and 13 of Wright et al. (2010) as well as Figure 2 of Mainzer et al. (2011), the $W 1$ and $W 2$ bands were specifically designed to probe the deep, $3.3 \mu \mathrm{m}$ $\mathrm{CH}_{4}$ absorption band in brown dwarfs and the region relatively free of opacity at $\sim 4.6 \mu \mathrm{m}$. Since the peak of the Planck function at low temperatures is in the mid-infrared, a large amount of flux emerges in the $4.6 \mu \mathrm{m}$ window, and this makes the $W 1-W 2$ colors of cool brown dwarfs extremely red (see Section 2.1). Such red colors, which are almost unique among astronomical sources, make the identification of cool brown dwarfs much easier.

WISE launched on 2009 December 14 and, after an in-orbit checkout, began surveying the sky on 2010 January 14. Its Sun-synchronous polar orbit around the Earth meant that each location along the ecliptic was observed a minimum of eight times, with larger numbers of re-visits occurring at locations nearer the ecliptic poles. WISE completed its first full pass of the sky on 2010 July 17 and its second pass on 2011 January 9. During this second pass, the outer, secondary tank depleted its cryogen on 2010 August 5, rendering the $W 4$ band unusable, and the inner, primary tank depleted its cryogen on 2010 September 30 , rendering the $W 3$ band unusable. Thus, this second full sky pass is partly missing bands $W 3$ and $W 4$. Fortunately, the bands most crucial for brown dwarf selection-W1 and $W 2$-were little affected by this cryogen exhaustion. WISE continued to collect data on a third, incomplete sky pass in bands $W 1$ and W2 until data acquisition was halted on 2011 January 31.

Preliminary processing of the data, including single-frame and co-added images and photometrically and astrometrically characterized detections, has been used to search for cold brown dwarf candidates, as described in detail below. This is the same version of the pipeline software that produced the WISE Preliminary Data Release, details of which can be found in the Explanatory Supplement. ${ }^{25}$ For a more detailed description of the WISE mission and data products, see Wright et al. (2010) and the NASA/IPAC Infrared Science Archive (IRSA; http://irsa.ipac.caltech.edu). Because processing of the data continues as of this writing, our candidate selection is ongoing, and only a fraction of our candidates has been followed up, it is not possible to estimate the sky coverage or volume surveyed for discoveries presented herein. However, objects discussed here can be added to the growing census of brown dwarfs in the Solar Neighborhood and can be used to place lower limits, as we do in Section 5.3 below, to the brown dwarf space density as a function of type or temperature. As such, this paper should be regarded as a progress report on the continuing WISE search for previously missed brown dwarfs in the Sun's immediate vicinity.

\subsection{Comparison to Known M, L, and T Dwarfs}

Before beginning the hunt for brown dwarfs, it is necessary to establish empirically the locus of known brown dwarfs in WISE color space and to understand what other kinds of astrophysical

\footnotetext{
25 See http://wise2.ipac.caltech.edu/docs/release/prelim/expsup/.
} 


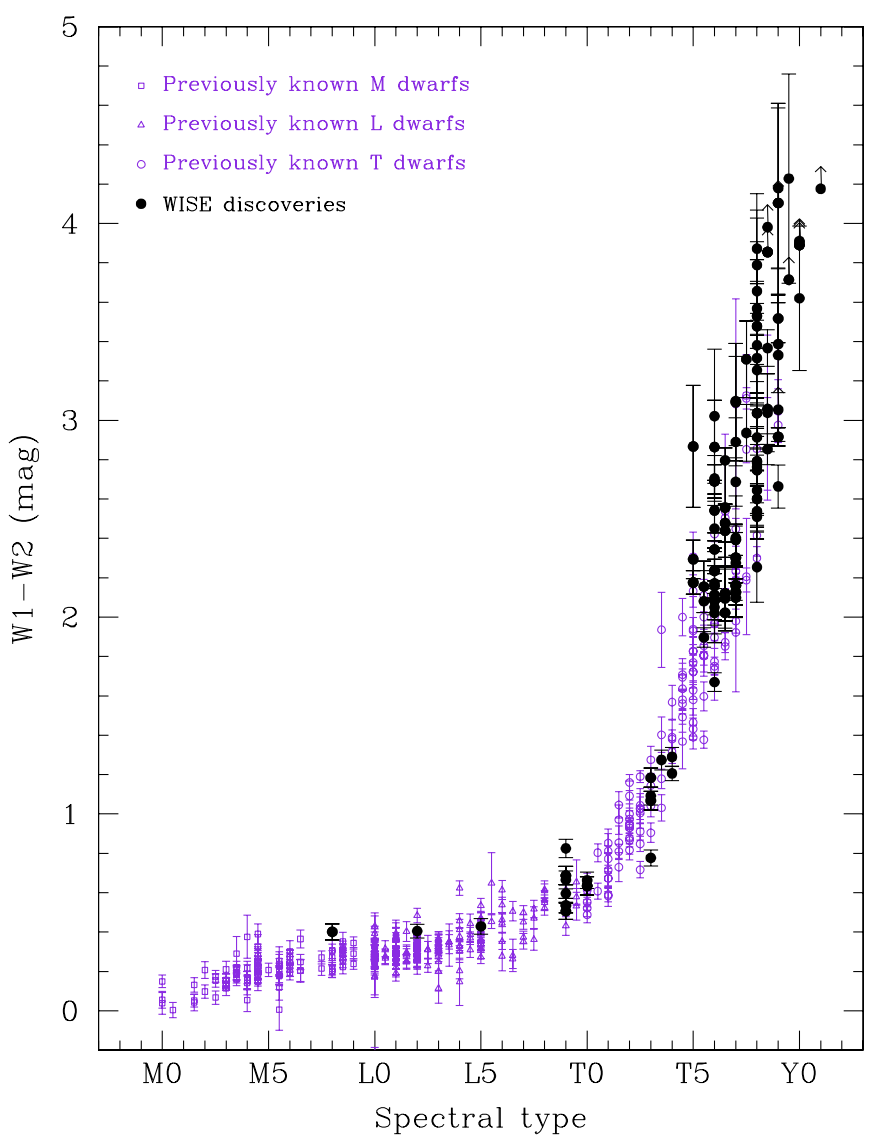

Figure 1. WISE $W 1-W 2$ color vs. spectral type. The $W 1-W 2$ colors for a sample of previously known M (open squares), L (open triangles), and $\mathrm{T}$ (open circles) dwarfs from Table 1 are shown in blue violet. The colors of new WISE discoveries from Table 2 are shown by the solid, black circles. $W 1-W 2$ color limits are indicated by arrows.

(A color version of this figure is available in the online journal.)

objects might fall in the same area. This will not only inform the search of WISE color space itself but also dictate the kinds of photometric follow-up that need to take place before timeintensive spectroscopic characterization begins.

We have performed a positional cross-correlation of nearby stars from Dwarf Archives ${ }^{26}$ against source lists derived from the WISE co-added data. Many of these stars are known to have substantial proper motion, so it was necessary to verify each cross-match by visually inspecting the WISE and Two Micron All-Sky Survey (2MASS; Skrutskie et al. 2006) images. The final cross-identifications are given in Table 1, which lists photometry $^{27}$ from 2MASS (when detected) and WISE for 118 previously cataloged T dwarfs, $142 \mathrm{~L}$ dwarfs, and $92 \mathrm{M}$ dwarfs. Figure 1 shows the resulting trend of WISE $W 1-W 2$ color as a function of spectral type for these objects, ranging from early-M through late-T. Note that there is a slow increase in the $W 1-W 2$ color between early-M and early-L, with the color stagnating near 0.3 mag between early- and mid-L. The $W 1-W 2$ color then rapidly increases at types later than mid-L, corresponding to the appearance of the methane fundamental band at $3.3 \mu \mathrm{m}$ (Noll et al. 2000). The average $W 1-W 2$ color is $\sim 0.6 \mathrm{mag}$ at T0 and $\sim 1.5 \mathrm{mag}$ at T5, with the color increasing to above 3.0 mag at late-T.

\footnotetext{
${ }^{26}$ See http://DwarfArchives.org.

27 In this and all subsequent tables, the errors listed are $1 \sigma$ values.
}

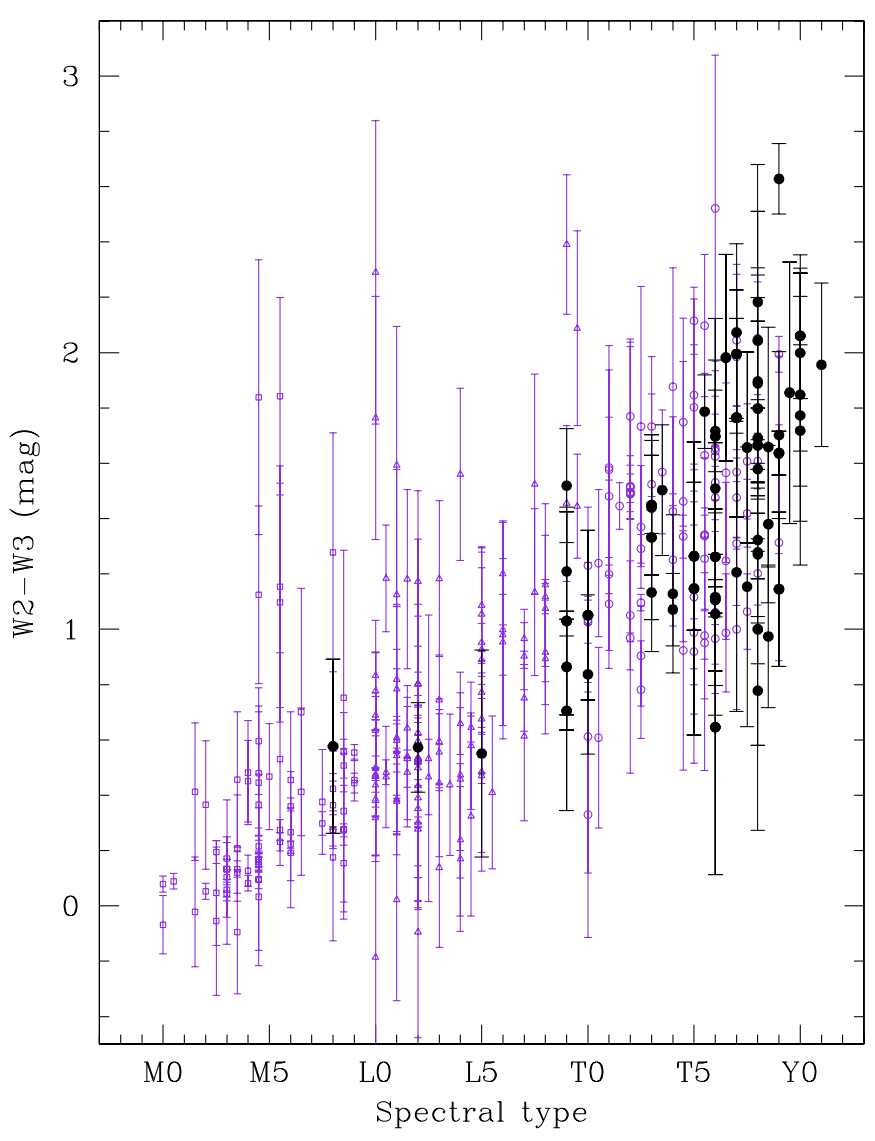

Figure 2. WISE $W 2-W 3$ color vs. spectral type. The color scheme is identical to that of Figure 1. For clarity, only those objects with detections in both $W 2$ and $W 3$ are shown.

(A color version of this figure is available in the online journal.)

The red $W 1-W 2$ colors $(>1.7 \mathrm{mag}$ ) of dwarfs of type mid-T and later are almost, but not entirely, unique among astrophysical sources. Dust-obscured galaxies (DOGs) and asymptotic giant branch stars (AGBs) are the major sources of contamination at these red $W 1-W 2$ colors, as analysis of the Spitzer Deep Wide-Field Survey (SDWFS) results of Eisenhardt et al. (2010) has shown. The three short-wavelength bands of WISE — which are close in wavelength to the 3.6, 4.5, and $8.0 \mu \mathrm{m}$ bands (hereafter denoted as $\operatorname{ch} 1, \operatorname{ch} 2$, and $\operatorname{ch} 4$, respectively) of the Infrared Array Camera (IRAC) on board the Spitzer Space Telescope - can help to distinguish between these populations. As Figure 1 of Eisenhardt et al. (2010) shows, most AGBs with very red $W 1-W 2$ (or $\operatorname{ch} 1-\operatorname{ch} 2$ ) colors can be distinguished by their very red $W 2-W 3$ (or ch $2-\operatorname{ch} 4$ ) colors. Brown dwarfs with similar $W 1-W 2$ colors are much bluer in $W 2-W 3$ color than these contaminants. Similarly, DOGs should be easily separable from brown dwarfs because, like AGBs, their $W 2-W 3$ (or ch $2-\operatorname{ch} 4$ ) colors tend to the red for objects with very red $W 1-W 2$ colors. This is further demonstrated in Figure 12 of Wright et al. (2010), where the bulk of the extragalactic menagerie, including red active galactic nuclei (AGNs), can be distinguished from cold brown dwarfs using a color of $W 2-W 3 \approx 2.5$ as the dividing line.

As with any set of generic color cuts, however, one should be ever vigilant for exceptions. As Figures 1 and 2 show, very late T dwarfs have colors approaching $W 2-W 3 \sim 2.0 \mathrm{mag}$, near the locus of extragalactic sources, but their $W 1-W 2$ colors are extreme $(>3.0 \mathrm{mag})$. Few extragalactic sources have 
WISE and Near-infrared Photometry for Known M, L, and T Dwarfs

\begin{tabular}{|c|c|c|c|c|c|c|c|c|c|c|}
\hline WISE Designation ${ }^{\mathrm{a}}$ & $\begin{array}{c}\text { Other Designation } \\
\text { (2) }\end{array}$ & $\begin{array}{l}\text { Disc. } \\
\text { Ref. } \\
\text { (3) }\end{array}$ & $\begin{array}{c}W 1 \\
(\mathrm{mag}) \\
(4)\end{array}$ & $\begin{array}{c}W 2 \\
(\mathrm{mag}) \\
(5)\end{array}$ & $\begin{array}{c}W 3 \\
(\mathrm{mag}) \\
(6)\end{array}$ & $\begin{array}{c}W 4 \\
(\mathrm{mag}) \\
(7)\end{array}$ & $\begin{array}{c}J \\
(\mathrm{mag}) \\
(8)\end{array}$ & $\begin{array}{c}H \\
(\mathrm{mag}) \\
(9)\end{array}$ & $\begin{array}{c}K_{s} \\
(\mathrm{mag}) \\
(10)\end{array}$ & $\begin{array}{c}\text { Spec. Ty. } \\
\text { (11) }\end{array}$ \\
\hline \multicolumn{11}{|c|}{ T Dwarfs: } \\
\hline WISEPC J003402.80-005207.4 & ULAS J003402.77-005206.7 & 1 & $17.320 \pm 0.249$ & $4.465 \pm$ & $>11.801$ & $>9.224$ & $18.150 \pm 0.030$ & $18.490 \pm 0.040$ & $18.480 \pm 0.050$ & $\mathrm{~T} 8.5$ \\
\hline WISEPC J005021.03-332229.2 & 2MASS J00501994-3322402 & 2 & $5.506 \pm$ & $3.526 \pm 0.036$ & $11.957 \pm 0.236$ & 89 & $928 \pm$ & 5.838 & 35 & $\mathrm{~T} 7$ \\
\hline WISEPC J005911.09-011400.6 & CFBDS J005910.90-011401.3 & 3 & $17.003 \pm 0.169$ & $13.668 \pm 0.044$ & $12.355 \pm 0.424$ & $>9.290$ & $8.060 \pm 0.030$ & $18.270 \pm 0.050$ & $18.630 \pm 0.050$ & $\mathrm{~T} 8.5$ \\
\hline WISEPC J013657.45+093347.0 & IPMS J013656.57+093347.3 & 4 & $11.967 \pm 0.025$ & $10.962 \pm 0.022$ & $9.671 \pm 0.047$ & $9.002 \pm 0.442$ & $13.455 \pm 0.030$ & $12.771 \pm 0.032$ & $12.562 \pm 0.024$ & $\mathrm{~T} 2.5$ \\
\hline WISEPA J015024.39+135924.3 & ULAS J015024.37+135924.0 & 5 & $17.392 \pm 0.265$ & $15.186 \pm 0.131$ & $>12.108$ & $>8.942$ & $17.730 \pm 0.020$ & $18.110 \pm 0.020$ & $17.840 \pm 0.160$ & $\mathrm{~T} 7.5$ \\
\hline WISEPA J015142.21+124429.8 & SDSS J015141.69+124429.6 & 6 & $14.595 \pm 0.039$ & $13.823 \pm 0.053$ & $12.246 \pm 0.445$ & $8.563 \pm 0.383$ & $16.566 \pm 0.129$ & $15.603 \pm 0.112$ & $15.183 \pm 0.189$ & $\mathrm{~T} 1$ \\
\hline WISEPC J020742.96+000056.9 & SDSS J020742.48+000056.2 & 6 & $16.403 \pm 0.097$ & $15.035 \pm 0.100$ & $>12.618$ & $>9.231$ & $16.799 \pm 0.156$ & $>16.396$ & $>15.412$ & T4.5 \\
\hline WISEPC J024313.48-245331.5 & 2MASSI J0243137-245329 & 7 & $14.680 \pm 0.035$ & $12.929 \pm 0.030$ & $11.285 \pm 0.131$ & $9.367 \pm 0.535$ & $15.381 \pm 0.050$ & $15.137 \pm 0.109$ & $15.216 \pm 0.168$ & T6 \\
\hline WISEPA J024749.98-163111.4 & SDSS J024749.90-163112.6 & 8 & $15.197 \pm 0.045$ & $14.197 \pm 0.054$ & $12.679 \pm 0.528$ & $>9.114$ & $17.186 \pm$ & $16.170 \pm$ & $15.616 \pm 0.193$ & T2: \\
\hline WISEPA J032553.11+042540.6 & SDSS J032553.17+042540.1 & 8 & $15.893 \pm 0.069$ & $13.783 \pm 0.045$ & $12.446 \pm 0.443$ & $>9.091$ & $16.254 \pm 0.137$ & $>16.080$ & $>16.525$ & $\mathrm{~T} 5.5$ \\
\hline
\end{tabular}

\section{Notes.}

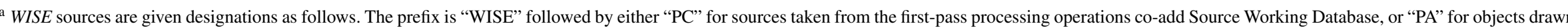

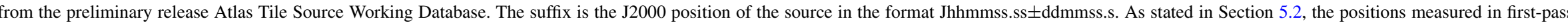
WISE processing and used to derive these designations should not be used for astrometric purposes.

b Special symbols on spectral types: “:” indicates an uncertain type; “::" indicates a highly uncertain type; “+” indicates that the spectrum is likely later than the type given.

${ }^{c}$ Previously identified, though unpublished, in 2MASS Prototype Camera Data as 2MASP J1007435+113432.

${ }^{\mathrm{d}}$ Previously identified, though unpublished, in 2MASS Prototype Camera Data as 2MASP J1520477+300210.

e Also known as PSS 1458+2839 (J. D. Kennefick 1995, private communication).

${ }^{\mathrm{f}}$ Object from J. D. Kennefick (1995, private communication).

g Luyten (1979a) quotes the discoverer as Hertzsprung.

h Object from W. E. Kunkel (1993, private communication).

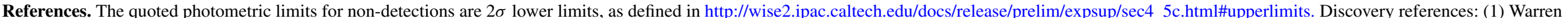

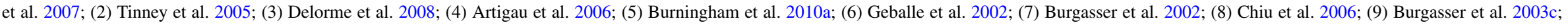

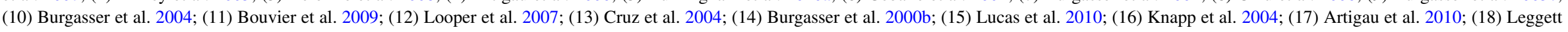

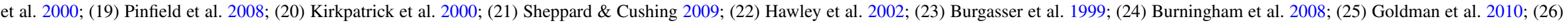

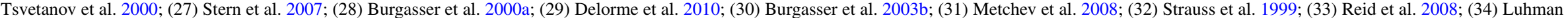

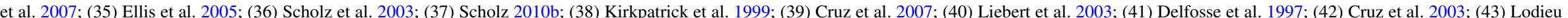

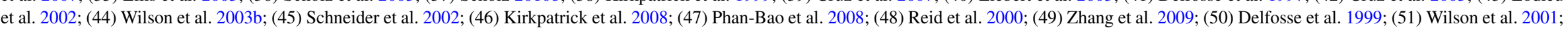

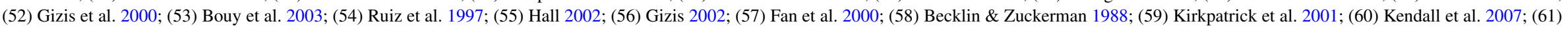

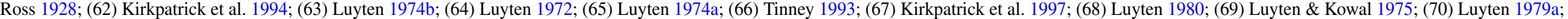

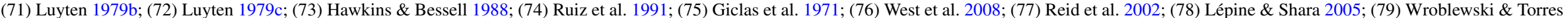
$1991 ;$ (80) this paper.

(This table is available in its entirety in a machine-readable form in the online journal. A portion is shown here for guidance regarding its form and content.) 


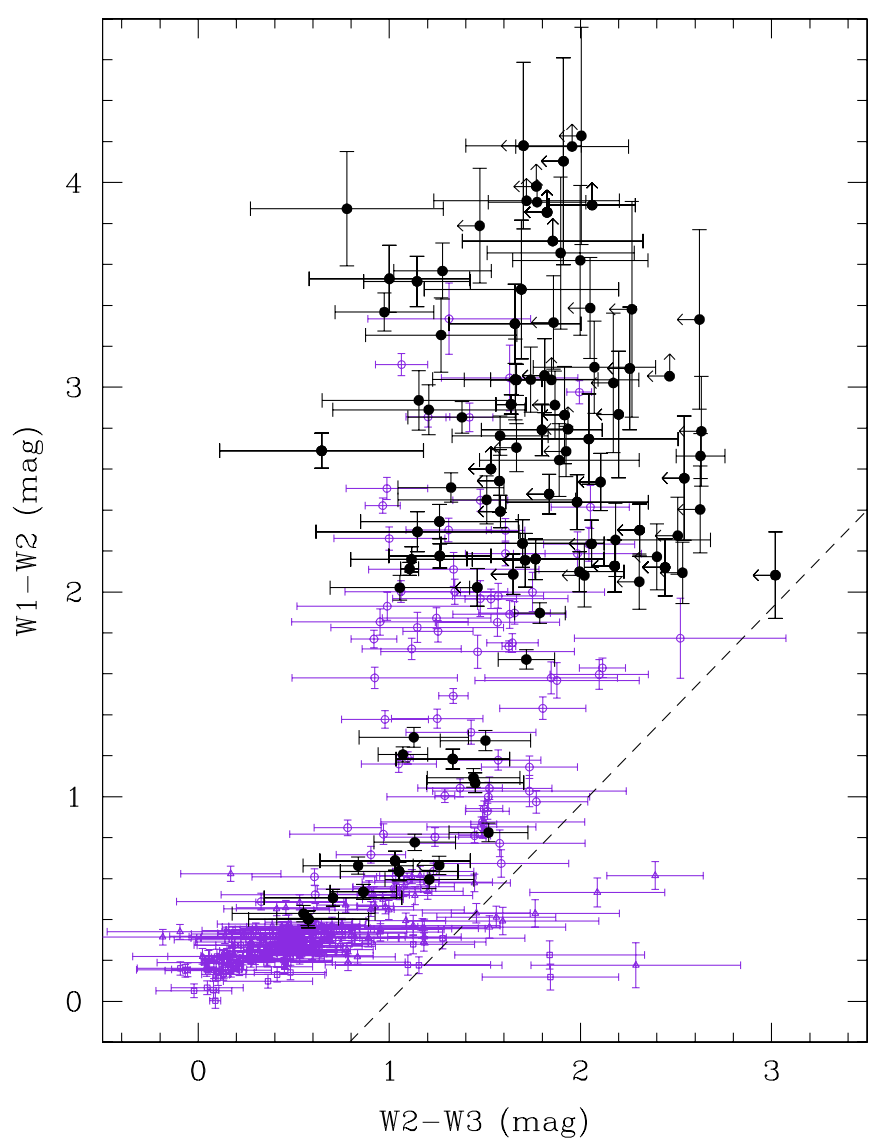

Figure 3. WISE color-color plot showing $W 1-W 2$ vs. $W 2-W 3$. Color coding is the same as in Figure 1. The dashed line indicates the criterion used, in search 2 (see Section 2.2), to eliminate extragalactic sources to the right of the line from the bulk of the M, L, and T dwarfs to the left.

(A color version of this figure is available in the online journal.)

$W 1-W 2$ colors this red, so the $W 2-W 3$ color criterion can be relaxed for the coldest objects (see Figure 3 ). Indeed, brown dwarfs with $T_{\text {eff }}<300 \mathrm{~K}$ are expected to turn to the red in $W 2-W 3$ color (Figure 14 of Wright et al. 2010). It should also be noted that the location of low-gravity or lowmetallicity brown dwarfs may not follow the general rule set by normal-gravity, solar-metallicity cases, so it is important to use other data (proper motion, parallax) when possible to identify brown dwarfs independent of photometric selections. Nonetheless, a number of possibly low-metallicity $\mathrm{T}$ dwarfs have been uncovered using these same photometric selections, as further discussed in the Appendix.

\subsection{Search for New Candidates}

We have used two different sets of criteria to search the WISE source lists for nearby brown dwarfs.

1. To find the coldest brown dwarfs, we selected high signalto-noise ( $\mathrm{S} / \mathrm{N}>3$ at $W 2$ ) detections having $W 1-W 2$ colors (or limits) greater than $1.5 \mathrm{mag}$, corresponding roughly to types $\geqslant \mathrm{T} 5$. (Because of the relative depths of the $W 1$ and $W 2$ bands, the $W 1-W 2$ requirement imposes a more severe $W 2 \mathrm{~S} / \mathrm{N}$ limit of its own, generally $W 2 \mathrm{~S} / \mathrm{N}>7$.) In order to assure that an object is real, we required it to have been detected at least eight times in the individual $W 2$ exposures; this eliminated spurious sources like cosmic rays and satellite trails that would otherwise not be eliminated during the outlier rejection step in co-add image creation (see Section IV.5.a.v of the Explanatory Supplement to the WISE Preliminary Data Release ${ }^{28}$ ). For our initial candidate selection, we also required $W 2-W 3<3.0 \mathrm{mag}$ if the object has a detection in $W 3$.

2. To find bright, nearby (i.e., high proper motion) L and $\mathrm{T}$ dwarfs that other surveys have missed, we searched for objects with $W 1-W 2$ colors greater than 0.4 mag (roughly types $\geqslant \mathrm{L} 5$ ), $W 2 \mathrm{~S} / \mathrm{N}$ values greater than 30 , and no association with a $2 \mathrm{MASS}$ source (implying that the $J-W 2$ color is either very red or the object has moved). It should be noted that the WISE source lists report 2MASS associations falling within 3 arcsec of each WISE source. ${ }^{29}$ As with the first search, we required the object to have been detected at least eight times in $W 2$ to assure its reliability. To eliminate extragalactic contaminants, we imposed one additional criterion that $W 1-W 2>0.96(W 2-W 3)-$ 0.96 (see dashed line in Figure 3). ${ }^{30}$

For both searches, no constraint on galactic latitude was imposed, although additional constraints were placed on object detections in order to assure that they were real, particularly for our earliest searches of the WISE source lists. First, the reduced $\chi^{2}$ value from the Point Spread Function photometric fitting ("rchi2" in the WISE Preliminary Release Source Catalog) was required to fall between 0.5 and 3.0 to assure that the source was pointlike. Second, the early version of the WISE data processing pipeline automatically flagged artifacts-bright star halos, diffraction spikes, latent images, ghost reflections from bright stars, etc. - only on individual frames and not on the co-added images. For search 2 above, because those objects all have high-S/N W2 detections, we are able to use these individual frame flags to remove objects marked as spurious. For fainter objects found in search 1, we created three-color images from the $W 1$ (blue), W2 (green), and W3 (red) co-added images, which were then inspected by eye to eliminate artifacts. Third, for all objects passing the above tests, we created finder charts showing the Digitized Sky Survey (DSS; $B, R, I$ ), the Sloan Digital Sky Survey (SDSS; York et al. 2000- $u, g, r, i$, and $z$, if available), 2MASS $\left(J, H, K_{s}\right)$, and WISE (W1,W2,W3,W4, + three-color image made from $W 1+W 2+W 3$ ) images. A visual inspection of these finder charts allowed us to remove other spurious sources and objects clearly visible in the short-wavelength optical bands, while also allowing us to check for proper motion between surveys for objects bright enough to have been detected at shorter wavelengths, such as $J$ and $H$ for brighter $\mathrm{T}$ dwarf candidates.

Example images are shown in Figure 4 for our spectroscopically confirmed candidates. WISE photometry for these same sources is given in Table 2 .

\footnotetext{
${ }_{28}$ Available at http://wise2.ipac.caltech.edu/docs/release/prelim/expsup/.

${ }^{29}$ See Section IV.7.a.i of http://wise2.ipac.caltech.edu/docs/release/ prelim/expsup/.

${ }^{30}$ It should be noted that five objects from Table 1 fall more than $1 \sigma$ to the right of this line. These objects are the M dwarfs CTI 064951.4+280442 and CTI 065950.5+280228 and the L dwarfs SDSS J082030.12+103737.0, SDSS J102947.68+483412.2, and SDSS J204317.69-155103.4. Visual inspection of the WISE images for each of these shows that the W3 detections for all five sources are almost certainly spurious, the photometry code having found a low-level $W 3$ "detection" at the sky location of the object. The signal-to-noise $(\mathrm{S} / \mathrm{N})$ values for each of these $W 3$ detections are 3.1, 2.2, 3.1, 2.0 , and 4.4 , respectively. Because the $W 3$ band is sensitive to extended structure within the Milky Way as well as background variations by bright stars and the Moon, low-level $W 3$ detections should be regarded with caution.
} 


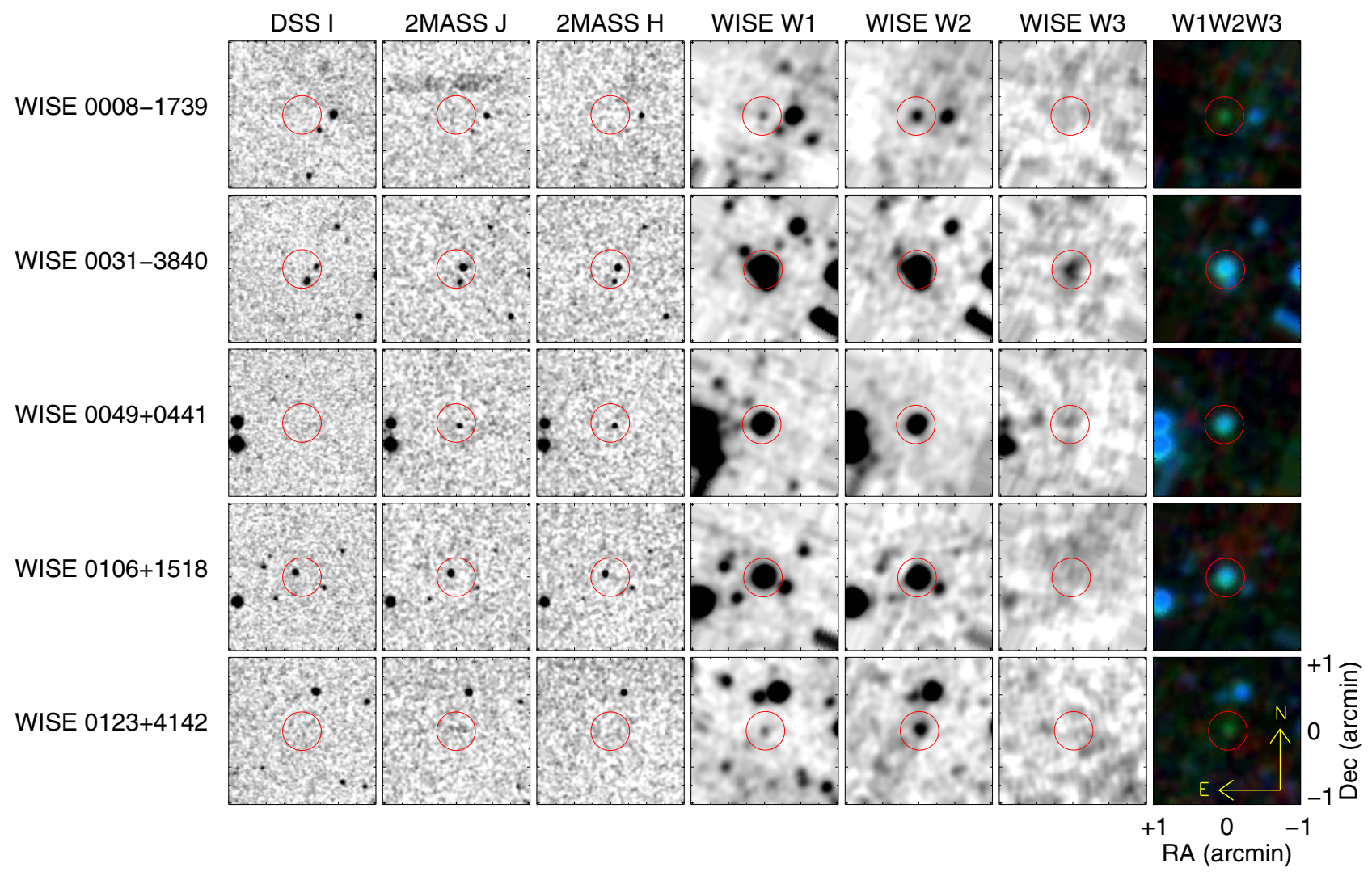

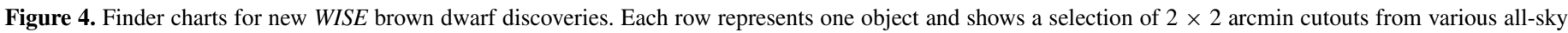

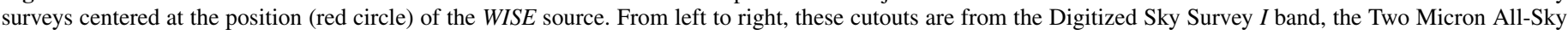

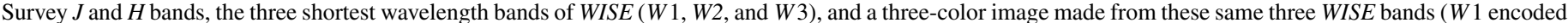

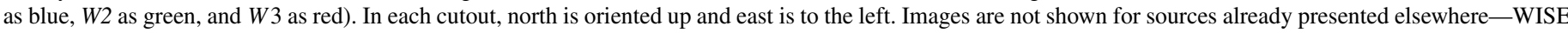

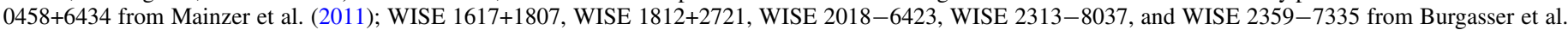
(2011a); and WISE 0148-7202, WISE 0410+1502, WISE 1405+5534, WISE 1541-2250, and WISE 2056+1459 from Cushing et al. (2011).

(An extended, color version of this figure is available in the online journal.)

\section{FOLLOW-UP IMAGING OBSERVATIONS}

\subsection{Ground-based Near-infrared Follow-up}

Follow-up imaging observations of WISE candidates are important not only for verifying that the source has the characteristics of a brown dwarf at shorter wavelengths but also for determining how bright the object is at wavelengths observable from the ground. This latter knowledge is necessary for determining which facility to use for spectroscopic confirmation.

Ground-based near-infrared observations at $J$ and $H$ bands are technically the easiest to acquire. Before discussing specifics, it should be noted that the two main near-infrared filter systems being used today-the 2MASS system and the Mauna Kea Observatories Near-infrared (MKO-NIR, or just MKO) system - will yield somewhat different results for the same objects. The 2MASS bandpasses are illustrated in Figure 2 of Skrutskie et al. (2006), and the MKO filter profiles are shown in Figure 1 of Tokunaga et al. (2002). A comparison of the two filter sets is illustrated in Figure 4 of Bessell (2005). For $J$ and $H$ bands, the main difference between the two systems lies in the width of the $J$-band filter; the $H$-band filters are very similar. The 2MASS $J$-band profile extends to bluer wavelengths than does the MKO $J$-band profile, and the overall shapes of the two $J$ filters are quite different, the 2MASS $J$ filter lacking the top-hat shape that is characteristic of most filter profiles. As a result, the measured $J$-band magnitude of a brown dwarf, whose spectral signature is also quite complex at these same wavelengths, can be considerably different between the two systems. This is dramatically illustrated in Figure 3 of Stephens \& Leggett (2004), which shows that although the $H$-band magnitudes are (as expected) very similar between the two systems for a wide range of brown dwarf types, the $J$-band magnitudes (and hence, $J-H$ colors) can differ by as much as 0.5 mag for late-type $\mathrm{T}$ dwarfs. In the discussion that follows, we note the systems on which our photometry was obtained and we mark photometry from the two systems with different colors and symbols on the plots.

As Figures 5 and 6 show, mid- to late-T dwarfs $(W 1-W 2>$ $1.5 \mathrm{mag}$ ) have relatively blue colors, $J-H \lesssim 0.4 \mathrm{mag}$, on the 2MASS filter system. The upper right-hand panel of Figure 5 of Leggett et al. (2010) shows the trend of $J-H$ color versus spectral type using the MKO filter system, and there we find that $J-H \lesssim-0.1$ mag for dwarfs $\geqslant \mathrm{T} 5$. These blue colors in both systems are a consequence of stronger methane bands and collision-induced $\mathrm{H}_{2}$ absorption at $H$ band compared to $J$ band. This color stands in contrast to the majority of other astrophysical sources, whose $J-H$ colors are much redder than this. For example, Figure 19 of Skrutskie et al. (2006) shows the 2MASS $J-H$ color distribution of detected sources at high Galactic latitude and confirms that most 2MASS objects have colors redder than those of mid- to late-T dwarfs. Nevertheless, there are true astrophysical sources-e.g., main-sequence stars earlier than type K0 (Table 2 of Bessell \& Brett 1988); certain AGNs, particularly those with $0.7<z<1.1$ (Figure 2 of 
Table 2

WISE Photometry for WISE Brown Dwarf Discoveries

\begin{tabular}{|c|c|c|c|c|c|c|c|c|c|}
\hline Object Name $^{\mathrm{a}}$ & $\begin{array}{l}\text { Disc. } \\
\text { Ref. } \\
(2)\end{array}$ & $\begin{array}{c}b \\
(\mathrm{deg}) \\
(3)\end{array}$ & $\begin{array}{c}W 1 \\
(\mathrm{mag}) \\
(4)\end{array}$ & $\begin{array}{c}W 2 \\
(\mathrm{mag}) \\
(5)\end{array}$ & $\begin{array}{c}\text { W3 } \\
\text { (mag) } \\
(6)\end{array}$ & $\begin{array}{c}W 4 \\
(\mathrm{mag}) \\
(7)\end{array}$ & $\begin{array}{c}W 1-W 2 \\
(\mathrm{mag}) \\
(8)\end{array}$ & $\begin{array}{c}W 2-W 3 \\
(\mathrm{mag}) \\
(9)\end{array}$ & $\begin{array}{c}\text { No. of WISE } \\
\text { Coverages } \\
\text { (10) }\end{array}$ \\
\hline WISEPC J000849.76-173922.6 & 1 & -76.3 & $16.593 \pm 0.114$ & $14.543 \pm 0.072$ & $>12.235$ & $8.928 \pm 0.381$ & $2.050 \pm 0.135$ & $<2.308$ & 13 \\
\hline WISEPC J003119.76-384036.4 & 1 & -77.7 & $12.433 \pm 0.026$ & $12.028 \pm 0.024$ & $11.455 \pm 0.160$ & $>8.763$ & $0.405 \pm 0.035$ & $0.574 \pm 0.162$ & 12 \\
\hline WISEPC J004928.48+044100.1 & 1 & -58.1 & $13.448 \pm 0.028$ & $12.942 \pm 0.030$ & $12.237 \pm 0.359$ & $8.677 \pm 0.286$ & $0.506 \pm 0.041$ & $0.705 \pm 0.360$ & 14 \\
\hline WISEPC J010637.07+151852.8 & 1 & -47.3 & $13.088 \pm 0.030$ & $12.687 \pm 0.028$ & $12.110 \pm 0.313$ & $9.105 \pm 0.465$ & $0.401 \pm 0.041$ & $0.577 \pm 0.314$ & 13 \\
\hline WISEPA J012333.21+414203.9 & 1 & -20.7 & $17.123 \pm 0.168$ & $14.848 \pm 0.086$ & $>12.339$ & $>8.839$ & $2.275 \pm 0.189$ & $<2.509$ & 13 \\
\hline WISEPC J013836.59-032221.2 & 1 & -63.7 & $14.427 \pm 0.034$ & $13.359 \pm 0.034$ & $11.910 \pm 0.252$ & $>8.957$ & $1.068 \pm 0.048$ & $1.449 \pm 0.254$ & 14 \\
\hline WISEPC J014807.25-720258.7 & 1,5 & -44.4 & $18.812 \pm 0.529$ & $14.584 \pm 0.051$ & $>12.579$ & $>9.521$ & $4.228 \pm 0.531$ & $<2.005$ & 24 \\
\hline WISEPA J015010.86+382724.3 & 1 & -22.9 & $13.619 \pm 0.028$ & $12.984 \pm 0.036$ & $11.933 \pm 0.305$ & $>8.779$ & $0.635 \pm 0.046$ & $1.051 \pm 0.307$ & 10 \\
\hline WISEPA J020625.26+264023.6 & 1 & -33.2 & $13.401 \pm 0.028$ & $12.805 \pm 0.035$ & $11.596 \pm 0.230$ & $>9.144$ & $0.596 \pm 0.045$ & $1.209 \pm 0.233$ & 11 \\
\hline WISEPA J022105.94+384202.9 & 1 & -20.9 & $16.715 \pm 0.130$ & $14.621 \pm 0.075$ & $>12.087$ & $>8.685$ & $2.094 \pm 0.150$ & $<2.534$ & 12 \\
\hline WISEPC J022322.39-293258.1 & 1 & -69.6 & $16.928 \pm 0.138$ & $13.992 \pm 0.044$ & $12.838 \pm 0.504$ & $>9.544$ & $2.936 \pm 0.145$ & $1.154 \pm 0.506$ & 15 \\
\hline WISEPA J022623.98-021142.8 & 1 & -56.2 & $17.635 \pm 0.291$ & $14.543 \pm 0.069$ & $>12.285$ & $>8.998$ & $3.092 \pm 0.299$ & $<2.258$ & 13 \\
\hline WISEPA J025409.45+022359.1 & 1 & -48.2 & $15.743 \pm 0.070$ & $12.707 \pm 0.031$ & $11.042 \pm 0.131$ & $>9.067$ & $3.036 \pm 0.077$ & $1.665 \pm 0.135$ & 11 \\
\hline WISEPA J030533.54+395434.4 & 1 & -16.0 & $16.815 \pm 0.143$ & $14.643 \pm 0.073$ & $>12.243$ & $>9.169$ & $2.172 \pm 0.161$ & $<2.400$ & 13 \\
\hline WISEPA J030724.57+290447.6 & 1 & -25.0 & $17.438 \pm 0.285$ & $14.882 \pm 0.103$ & $>12.339$ & $>8.977$ & $2.556 \pm 0.303$ & $<2.543$ & 11 \\
\hline WISEPA J031325.96+780744.2 & 1 & 17.2 & $16.087 \pm 0.070$ & $13.234 \pm 0.034$ & $11.854 \pm 0.282$ & $>8.760$ & $2.853 \pm 0.078$ & $1.380 \pm 0.284$ & 16 \\
\hline WISEPC J032337.53-602554.9 & 1 & -47.9 & $17.504 \pm 0.190$ & $14.466 \pm 0.054$ & $12.807 \pm 0.430$ & $>9.287$ & $3.038 \pm 0.198$ & $1.659 \pm 0.433$ & 17 \\
\hline WISEPC J033349.34-585618.7 & 1 & -47.6 & $14.024 \pm 0.028$ & $13.247 \pm 0.029$ & $12.114 \pm 0.212$ & $>9.746$ & $0.777 \pm 0.040$ & $1.133 \pm 0.214$ & 20 \\
\hline WISEPA J041022.71+150248.5 & 1 & -25.9 & $>18.101$ & $14.190 \pm 0.059$ & $12.472 \pm 0.482$ & $>8.923$ & $>3.911$ & $1.718 \pm 0.486$ & 12 \\
\hline WISEPA J041054.48+141131.6 & 1 & -26.3 & $17.103 \pm 0.186$ & $15.021 \pm 0.102$ & $>12.001$ & $>9.069$ & $2.082 \pm 0.212$ & $<3.020$ & 12 \\
\hline WISEPA J044853.29-193548.5 & 1 & -35.4 & $16.483 \pm 0.086$ & $14.189 \pm 0.046$ & $13.042 \pm 0.528$ & $>8.950$ & $2.294 \pm 0.098$ & $1.147 \pm 0.530$ & 19 \\
\hline WISEPA J045853.89+643452.9 & 2 & 13.3 & $16.370 \pm 0.088$ & $13.003 \pm 0.030$ & $12.029 \pm 0.256$ & $>9.260$ & $3.367 \pm 0.093$ & $0.974 \pm 0.258$ & 15 \\
\hline WISEPA J050003.05-122343.2 & 1 & -30.2 & $17.762 \pm 0.276$ & $13.973 \pm 0.046$ & $>12.500$ & $>8.845$ & $3.789 \pm 0.280$ & $<1.473$ & 16 \\
\hline WISEPA J051317.28+060814.7 & 1 & -18.5 & $15.856 \pm 0.079$ & $13.834 \pm 0.047$ & $>12.374$ & $>9.118$ & $2.022 \pm 0.092$ & $<1.460$ & 11 \\
\hline WISEPA J052536.33+673952.3 & 1 & 17.3 & $17.920 \pm 0.330$ & $14.899 \pm 0.083$ & $>12.727$ & $>9.128$ & $3.021 \pm 0.340$ & $<2.172$ & 16 \\
\hline WISEPA J052844.51-330823.9 & 1 & -30.8 & $17.632 \pm 0.219$ & $14.534 \pm 0.055$ & $12.461 \pm 0.316$ & $>9.312$ & $3.098 \pm 0.226$ & $2.073 \pm 0.321$ & 18 \\
\hline WISEPA J053957.02-103436.5 & 1 & -20.6 & $16.845 \pm 0.134$ & $14.764 \pm 0.077$ & $>12.743$ & $>9.202$ & $2.081 \pm 0.155$ & $<2.021$ & 14 \\
\hline WISEPA J054231.26-162829.1 & 1 & -22.4 & $16.385 \pm 0.087$ & $13.907 \pm 0.043$ & $>12.071$ & $>9.068$ & $2.478 \pm 0.097$ & $<1.836$ & 15 \\
\hline WISEPA J061135.13-041024.0 & 1 & -10.7 & $13.554 \pm 0.029$ & $12.891 \pm 0.030$ & $12.054 \pm 0.286$ & $>8.818$ & $0.663 \pm 0.042$ & $0.837 \pm 0.288$ & 13 \\
\hline WISEPA J061213.93-303612.7 & 1 & -21.2 & $16.586 \pm 0.147$ & $14.044 \pm 0.045$ & $>12.468$ & $>8.954$ & $2.542 \pm 0.154$ & $<1.576$ & 18 \\
\hline WISEPA J061208.69-492023.8 & 1 & -26.4 & $15.349 \pm 0.036$ & $14.075 \pm 0.034$ & $12.572 \pm 0.234$ & $>9.720$ & $1.274 \pm 0.050$ & $1.503 \pm 0.236$ & 44 \\
\hline WISEPA J061407.49+391236.4 & 1 & 10.1 & $16.338 \pm 0.110$ & $13.633 \pm 0.039$ & $>11.968$ & $>8.960$ & $2.705 \pm 0.117$ & $<1.665$ & 13 \\
\hline WISEPA J062309.94-045624.6 & 1 & -8.5 & $17.036 \pm 0.177$ & $13.781 \pm 0.043$ & $12.510 \pm 0.394$ & $>8.661$ & $3.255 \pm 0.182$ & $1.271 \pm 0.396$ & 16 \\
\hline WISEPA J062542.21+564625.5 & 1 & 19.0 & $16.558 \pm 0.102$ & $14.321 \pm 0.056$ & $12.624 \pm 0.423$ & $>8.886$ & $2.237 \pm 0.116$ & $1.697 \pm 0.427$ & 15 \\
\hline WISEPA J062720.07-111428.8 & 1 & -10.4 & $14.897 \pm 0.038$ & $13.227 \pm 0.029$ & $11.510 \pm 0.145$ & $>9.206$ & $1.670 \pm 0.048$ & $1.717 \pm 0.148$ & 26 \\
\hline WISEPA J065609.60+420531.0 & 1 & 18.6 & $14.318 \pm 0.032$ & $13.226 \pm 0.033$ & $11.786 \pm 0.240$ & $>8.846$ & $1.092 \pm 0.046$ & $1.440 \pm 0.242$ & 14 \\
\hline WISEPA J074457.15+562821.8 & 1 & 29.5 & $17.136 \pm 0.168$ & $14.492 \pm 0.059$ & $12.603 \pm 0.414$ & $>9.091$ & $2.644 \pm 0.178$ & $1.889 \pm 0.418$ & 15 \\
\hline WISEPA J075003.84+272544.8 & 1 & 24.2 & $>18.338$ & $14.483 \pm 0.070$ & $>12.658$ & $>8.765$ & $>3.855$ & $<1.825$ & 12 \\
\hline WISEPA J075108.79-763449.6 & 1 & -22.9 & $17.129 \pm 0.102$ & $14.465 \pm 0.040$ & $11.837 \pm 0.121$ & $>9.306$ & $2.664 \pm 0.110$ & $2.628 \pm 0.127$ & 45 \\
\hline WISEPC J075946.98-490454.0 & 1 & -9.9 & $17.680 \pm 0.276$ & $13.808 \pm 0.038$ & $13.030 \pm 0.503$ & $>9.120$ & $3.872 \pm 0.279$ & $0.778 \pm 0.504$ & 19 \\
\hline WISEPA J081958.05-033529.0 & 1 & 17.8 & $14.356 \pm 0.034$ & $13.066 \pm 0.034$ & $11.938 \pm 0.284$ & $>8.926$ & $1.290 \pm 0.048$ & $1.128 \pm 0.286$ & 13 \\
\hline
\end{tabular}


Table 2

Continued)

\begin{tabular}{|c|c|c|c|c|c|c|c|c|c|}
\hline Object Name $^{a}$ & $\begin{array}{l}\text { Disc. } \\
\text { Ref. } \\
(2) \\
\end{array}$ & $\begin{array}{c}b \\
(\mathrm{deg}) \\
(3)\end{array}$ & $\begin{array}{c}W 1 \\
(\mathrm{mag}) \\
(4)\end{array}$ & $\begin{array}{c}W 2 \\
(\mathrm{mag}) \\
(5)\end{array}$ & $\begin{array}{c}W 3 \\
(\mathrm{mag}) \\
(6)\end{array}$ & $\begin{array}{c}W 4 \\
(\mathrm{mag}) \\
(7)\end{array}$ & $\begin{array}{c}W 1-W 2 \\
(\mathrm{mag}) \\
(8)\end{array}$ & $\begin{array}{c}W 2-W 3 \\
(\mathrm{mag}) \\
(9)\end{array}$ & $\begin{array}{c}\text { No. of WISE } \\
\text { Coverages } \\
\text { (10) }\end{array}$ \\
\hline WISEPA J082131.63+144319.3 & 1 & 26.4 & $16.438 \pm 0.114$ & $14.283 \pm 0.064$ & $>12.572$ & $>8.893$ & $2.155 \pm 0.131$ & $<1.711$ & 10 \\
\hline WISEPC J083641.12-185947.2 & 1 & 12.9 & $18.405 \pm 0.520$ & $15.024 \pm 0.085$ & $>12.755$ & $>9.156$ & $3.381 \pm 0.527$ & $<2.269$ & 15 \\
\hline WISEPA J085716.25+560407.6 & 1 & 39.6 & $17.068 \pm 0.153$ & $14.031 \pm 0.046$ & $>12.291$ & $>9.270$ & $3.037 \pm 0.160$ & $<1.740$ & 15 \\
\hline WISEPA J090649.36+473538.6 & 1 & 42.1 & $17.342 \pm 0.209$ & $14.595 \pm 0.071$ & $12.551 \pm 0.461$ & $>8.746$ & $2.747 \pm 0.221$ & $2.044 \pm 0.466$ & 12 \\
\hline WISEPC J092906.77+040957.9 & 1 & 36.6 & $16.550 \pm 0.122$ & $14.111 \pm 0.056$ & $12.129 \pm 0.369$ & $>9.140$ & $2.439 \pm 0.134$ & $1.982 \pm 0.373$ & 10 \\
\hline WISEPC J095259.29+195507.3 & 1 & 48.7 & $17.249 \pm 0.228$ & $14.385 \pm 0.067$ & $>12.468$ & $>9.183$ & $2.864 \pm 0.238$ & $<1.917$ & 11 \\
\hline WISEPC J101808.05-244557.7 & 1 & 26.2 & $17.001 \pm 0.159$ & $14.088 \pm 0.047$ & $>12.222$ & $>8.771$ & $2.913 \pm 0.166$ & $<1.866$ & 14 \\
\hline WISEPA J101905.63+652954.2 & 1 & 44.8 & $16.285 \pm 0.073$ & $13.941 \pm 0.040$ & $12.679 \pm 0.411$ & $>9.147$ & $2.344 \pm 0.083$ & $1.262 \pm 0.413$ & 18 \\
\hline WISEPC J104245.23-384238.3 & 1 & 17.6 & $>18.496$ & $14.515 \pm 0.060$ & $>12.746$ & $>9.099$ & $>3.981$ & $<1.769$ & 14 \\
\hline WISEPC J112254.73+255021.5 & 1 & 70.1 & $16.051 \pm 0.084$ & $13.965 \pm 0.052$ & $>12.317$ & $>9.429$ & $2.086 \pm 0.099$ & $<1.648$ & 9 \\
\hline WISEPC J115013.88+630240.7 & 1 & 52.7 & $16.993 \pm 0.133$ & $13.425 \pm 0.034$ & $12.147 \pm 0.253$ & $>8.831$ & $3.568 \pm 0.137$ & $1.278 \pm 0.255$ & 16 \\
\hline WISEPC J121756.91+162640.2 & 1 & 76.7 & $16.591 \pm 0.118$ & $13.074 \pm 0.034$ & $11.929 \pm 0.277$ & $>8.710$ & $3.517 \pm 0.123$ & $1.145 \pm 0.279$ & 11 \\
\hline WISEPC J131106.24+012252.4 & 1 & 63.8 & $18.064 \pm 0.431$ & $14.733 \pm 0.080$ & $>12.110$ & $>9.287$ & $3.331 \pm 0.438$ & $<2.623$ & 12 \\
\hline WISEPC J131141.91+362925.2 & 1 & 79.7 & $13.496 \pm 0.027$ & $13.067 \pm 0.030$ & $12.516 \pm 0.373$ & $>8.830$ & $0.429 \pm 0.040$ & $0.551 \pm 0.374$ & 15 \\
\hline WISEPC J132004.16+603426.2 & 1 & 56.2 & $16.609 \pm 0.121$ & $14.489 \pm 0.070$ & $>12.046$ & $>9.087$ & $2.120 \pm 0.140$ & $<2.443$ & 9 \\
\hline WISEPA J132233.66-234017.1 & 1,4 & 38.6 & $17.192 \pm 0.222$ & $13.876 \pm 0.052$ & $>12.017$ & $8.594 \pm 0.340$ & $3.316 \pm 0.228$ & $<1.859$ & 9 \\
\hline WISEPC J134806.99+660327.8 & 1 & 50.0 & $14.441 \pm 0.030$ & $13.754 \pm 0.036$ & $12.724 \pm 0.393$ & $>9.760$ & $0.687 \pm 0.047$ & $1.030 \pm 0.395$ & 18 \\
\hline WISEPC J140518.40+553421.4 & 1,5 & 58.5 & $>17.989$ & $14.085 \pm 0.041$ & $12.312 \pm 0.252$ & $>9.115$ & $>3.904$ & $1.773 \pm 0.255$ & 21 \\
\hline WISEPA J143602.19-181421.8 & 1 & 38.0 & $16.904 \pm 0.159$ & $14.650 \pm 0.082$ & $12.467 \pm 0.491$ & $>8.799$ & $2.254 \pm 0.179$ & $2.183 \pm 0.498$ & 12 \\
\hline WISEPC J145715.03+581510.2 & 1 & 51.9 & $16.541 \pm 0.086$ & $14.380 \pm 0.050$ & $12.615 \pm 0.355$ & $>9.558$ & $2.161 \pm 0.099$ & $1.765 \pm 0.359$ & 18 \\
\hline WISEPC J150649.97+702736.0 & 1 & 42.6 & $13.390 \pm 0.025$ & $11.277 \pm 0.020$ & $10.171 \pm 0.043$ & $9.889 \pm 0.498$ & $2.113 \pm 0.032$ & $1.106 \pm 0.047$ & 25 \\
\hline WISEPC J151906.64+700931.5 & 1 & 42.0 & $17.594 \pm 0.160$ & $14.064 \pm 0.035$ & $13.064 \pm 0.418$ & $>10.043$ & $3.530 \pm 0.164$ & $1.000 \pm 0.419$ & 29 \\
\hline WISEPA J154151.66-225025.2 & 1.5 & 25.2 & $>17.018$ & $13.982 \pm 0.112$ & $12.134 \pm 0.443$ & $>9.064$ & $>3.036$ & $1.848 \pm 0.457$ & 11 \\
\hline WISEPA J161215.94-342027.1 & 1 & 12.3 & $>16.881$ & $14.085 \pm 0.077$ & $>12.150$ & $>8.744$ & $>2.796$ & $<1.935$ & 13 \\
\hline WISEPA J161441.45+173936.7 & 1,4 & 42.3 & $18.333 \pm 0.503$ & $14.229 \pm 0.053$ & $>12.318$ & $>9.151$ & $4.104 \pm 0.506$ & $<1.911$ & 14 \\
\hline WISEPA J161705.75+180714.3 & 3 & 42.0 & $16.831 \pm 0.117$ & $14.039 \pm 0.046$ & $12.241 \pm 0.312$ & $>9.255$ & $2.792 \pm 0.126$ & $1.798 \pm 0.315$ & 16 \\
\hline WISEPA J162208.94-095934.6 & 1 & 26.8 & $16.251 \pm 0.102$ & $14.016 \pm 0.054$ & $>11.958$ & $>8.760$ & $2.235 \pm 0.115$ & $<2.058$ & 11 \\
\hline WISEPA J162725.64+325525.5 & 1,4 & 43.3 & $16.303 \pm 0.077$ & $13.613 \pm 0.036$ & $12.967 \pm 0.532$ & $>9.197$ & $2.690 \pm 0.085$ & $0.646 \pm 0.533$ & 18 \\
\hline WISEPA J164715.59+563208.2 & 1 & 39.3 & $13.603 \pm 0.026$ & $13.068 \pm 0.026$ & $12.204 \pm 0.171$ & $>9.208$ & $0.535 \pm 0.037$ & $0.864 \pm 0.173$ & 41 \\
\hline WISEPA J165311.05+444423.9 & 1,4 & 39.2 & $16.580 \pm 0.089$ & $13.817 \pm 0.036$ & $12.238 \pm 0.249$ & $>9.517$ & $2.763 \pm 0.096$ & $1.579 \pm 0.252$ & 23 \\
\hline WISEPA J171104.60+350036.8 & 1 & 34.8 & $18.267 \pm 0.367$ & $14.611 \pm 0.056$ & $12.715 \pm 0.381$ & $>9.454$ & $3.656 \pm 0.371$ & $1.896 \pm 0.385$ & 21 \\
\hline WISEPA J171717.02+612859.3 & 1 & 34.7 & $18.436 \pm 0.334$ & $14.958 \pm 0.056$ & $13.267 \pm 0.505$ & $>9.142$ & $3.478 \pm 0.339$ & $1.691 \pm 0.508$ & 36 \\
\hline WISEPA J172844.93+571643.6 & 1 & 33.6 & $17.368 \pm 0.106$ & $14.918 \pm 0.049$ & $13.409 \pm 0.462$ & $>9.899$ & $2.450 \pm 0.117$ & $1.509 \pm 0.465$ & 52 \\
\hline WISEPA J173835.53+273258.9 & 1,5 & 27.2 & $18.155 \pm 0.362$ & $14.535 \pm 0.057$ & $12.536 \pm 0.350$ & $>9.182$ & $3.620 \pm 0.366$ & $1.999 \pm 0.355$ & 18 \\
\hline WISEPA J174124.26+255319.5 & 1,4 & 26.1 & $15.228 \pm 0.040$ & $12.312 \pm 0.025$ & $10.675 \pm 0.075$ & $>8.580$ & $2.916 \pm 0.047$ & $1.637 \pm 0.079$ & 17 \\
\hline WISEPA J180435.40+311706.1 & 1 & 23.0 & $>18.423$ & $14.709 \pm 0.062$ & $12.854 \pm 0.468$ & $>9.391$ & $>3.714$ & $1.855 \pm 0.472$ & 19 \\
\hline WISEPA J181210.85+272144.3 & 3 & 20.1 & $17.238 \pm 0.173$ & $14.181 \pm 0.050$ & $>12.369$ & $>9.284$ & $3.057 \pm 0.180$ & $<1.812$ & 15 \\
\hline WISEPA J182831.08+265037.8 & 1,5 & 16.5 & $>18.452$ & $14.276 \pm 0.050$ & $12.320 \pm 0.291$ & $9.147 \pm 0.438$ & $>4.176$ & $1.956 \pm 0.295$ & 18 \\
\hline WISEPA J183058.57+454257.9 & 1 & 22.4 & $14.759 \pm 0.029$ & $14.094 \pm 0.034$ & $>12.833$ & $>9.286$ & $0.665 \pm 0.045$ & $<1.261$ & 42 \\
\hline WISEPA J184124.74+700038.0 & 1,4 & 26.2 & $16.485 \pm 0.050$ & $14.309 \pm 0.032$ & $13.045 \pm 0.265$ & $>9.424$ & $2.176 \pm 0.059$ & $1.264 \pm 0.267$ & 77 \\
\hline WISEPA J185215.78+353716.3 & 1 & 15.2 & $16.261 \pm 0.087$ & $14.162 \pm 0.044$ & $12.167 \pm 0.228$ & $9.405 \pm 0.489$ & $2.099 \pm 0.097$ & $1.995 \pm 0.232$ & 21 \\
\hline
\end{tabular}


Table 2
(Continued)

\begin{tabular}{|c|c|c|c|c|c|c|c|c|c|}
\hline Object Name $^{\mathrm{a}}$ & $\begin{array}{l}\text { Disc. } \\
\text { Ref. } \\
(2)\end{array}$ & $\begin{array}{c}b \\
(\mathrm{deg}) \\
(3)\end{array}$ & $\begin{array}{c}W 1 \\
(\mathrm{mag}) \\
(4)\end{array}$ & $\begin{array}{c}W 2 \\
(\mathrm{mag}) \\
(5)\end{array}$ & $\begin{array}{c}W 3 \\
\text { (mag) } \\
(6)\end{array}$ & $\begin{array}{c}W 4 \\
(\mathrm{mag}) \\
(7)\end{array}$ & $\begin{array}{c}W 1-W 2 \\
(\mathrm{mag}) \\
(8)\end{array}$ & $\begin{array}{c}W 2-W 3 \\
(\mathrm{mag}) \\
(9)\end{array}$ & $\begin{array}{c}\text { No. of WISE } \\
\text { Coverages } \\
\text { (10) }\end{array}$ \\
\hline WISEPA J190624.75+450808.2 & 1 & 16.3 & $15.978 \pm 0.052$ & $13.819 \pm 0.033$ & $12.703 \pm 0.317$ & $>9.480$ & $2.159 \pm 0.062$ & $1.116 \pm 0.319$ & 30 \\
\hline WISEPA J195246.66+724000.8 & 1 & 21.4 & $14.201 \pm 0.027$ & $12.995 \pm 0.025$ & $11.924 \pm 0.128$ & $>9.141$ & $1.206 \pm 0.037$ & $1.071 \pm 0.130$ & 47 \\
\hline WISEPA J195905.66-333833.7 & 1 & -27.9 & $16.419 \pm 0.135$ & $13.818 \pm 0.048$ & $>12.287$ & $>8.789$ & $2.601 \pm 0.143$ & $<1.531$ & 13 \\
\hline WISEPA J201824.96-742325.9 & 3 & -31.8 & $16.609 \pm 0.115$ & $13.719 \pm 0.041$ & $12.513 \pm 0.501$ & $>8.977$ & $2.890 \pm 0.122$ & $1.206 \pm 0.503$ & 12 \\
\hline WISEPC J205628.90+145953.3 & 1,5 & -19.1 & $>17.742$ & $13.852 \pm 0.043$ & $11.791 \pm 0.222$ & $>8.646$ & $>3.890$ & $2.061 \pm 0.226$ & 12 \\
\hline WISEPA J213456.73-713743.6 & 1 & -38.0 & $18.124 \pm 0.404$ & $13.944 \pm 0.045$ & $12.242 \pm 0.299$ & $>8.830$ & $4.180 \pm 0.406$ & $1.702 \pm 0.302$ & 13 \\
\hline WISEPC J215751.38+265931.4 & 1 & -21.6 & $16.956 \pm 0.193$ & $14.553 \pm 0.088$ & $>11.927$ & $>8.441$ & $2.403 \pm 0.212$ & $<2.626$ & 8 \\
\hline WISEPC J220922.10-273439.5 & 1 & -54.1 & $16.473 \pm 0.113$ & $13.786 \pm 0.048$ & $>11.861$ & $>9.124$ & $2.687 \pm 0.123$ & $<1.925$ & 10 \\
\hline WISEPC J221354.69+091139.4 & 1 & -37.3 & $16.635 \pm 0.111$ & $14.508 \pm 0.064$ & $>12.329$ & $>9.155$ & $2.127 \pm 0.128$ & $<2.179$ & 14 \\
\hline WISEPC J222623.05+044003.9 & 1 & -42.6 & $17.410 \pm 0.255$ & $14.625 \pm 0.082$ & $>11.992$ & $>9.075$ & $2.785 \pm 0.268$ & $<2.633$ & 11 \\
\hline WISEPC J223729.53-061434.2 & 1 & -51.9 & $17.527 \pm 0.297$ & $14.660 \pm 0.088$ & $>12.460$ & $>8.854$ & $2.867 \pm 0.310$ & $<2.200$ & 10 \\
\hline WISEPC J223937.55+161716.2 & 1 & -36.0 & $14.621 \pm 0.034$ & $13.437 \pm 0.035$ & $12.105 \pm 0.295$ & $>9.173$ & $1.184 \pm 0.049$ & $1.332 \pm 0.297$ & 12 \\
\hline WISEPC J225540.74-311841.8 & 1 & -64.4 & $16.617 \pm 0.129$ & $14.080 \pm 0.055$ & $>11.975$ & $>9.165$ & $2.537 \pm 0.140$ & $<2.105$ & 10 \\
\hline WISEPA J231336.40-803700.3 & 3 & -35.5 & $16.187 \pm 0.063$ & $13.677 \pm 0.034$ & $12.354 \pm 0.275$ & $>9.312$ & $2.510 \pm 0.072$ & $1.323 \pm 0.277$ & 22 \\
\hline WISEPC J231939.13-184404.3 & 1 & -67.3 & $17.043 \pm 0.187$ & $13.733 \pm 0.051$ & $12.076 \pm 0.341$ & $8.971 \pm 0.467$ & $3.310 \pm 0.194$ & $1.657 \pm 0.345$ & 11 \\
\hline WISEPC J232519.54-410534.9 & 1 & -67.4 & $17.499 \pm 0.242$ & $14.112 \pm 0.051$ & $>12.061$ & $>9.251$ & $3.387 \pm 0.247$ & $<2.051$ & 12 \\
\hline WISEPC J232728.75-273056.5 & 1 & -71.3 & $14.031 \pm 0.031$ & $13.206 \pm 0.034$ & $11.687 \pm 0.203$ & $>9.283$ & $0.825 \pm 0.046$ & $1.519 \pm 0.206$ & 12 \\
\hline WISEPC J234026.62-074507.2 & 1 & -64.3 & $15.951 \pm 0.070$ & $13.558 \pm 0.037$ & $>11.977$ & $>9.477$ & $2.393 \pm 0.079$ & $<1.581$ & 11 \\
\hline WISEPA J234351.20-741847.0 & 1 & -42.0 & $15.710 \pm 0.050$ & $13.689 \pm 0.035$ & $12.634 \pm 0.364$ & $>9.405$ & $2.021 \pm 0.061$ & $1.055 \pm 0.366$ & 19 \\
\hline WISEPC J234446.25+103415.8 & 1 & -48.9 & $>17.949$ & $14.895 \pm 0.105$ & $>12.429$ & $>8.543$ & $>3.054$ & $<2.466$ & 10 \\
\hline WISEPC J234841.10-102844.4 & 1 & -67.7 & $16.570 \pm 0.114$ & $14.268 \pm 0.057$ & $>11.958$ & $>9.525$ & $2.302 \pm 0.127$ & $<2.310$ & 13 \\
\hline WISEPA J235941.07-733504.8 & 3 & -43.0 & $15.166 \pm 0.039$ & $13.269 \pm 0.031$ & $11.482 \pm 0.129$ & $>9.446$ & $1.897 \pm 0.050$ & $1.787 \pm 0.133$ & 18 \\
\hline
\end{tabular}

Notes.

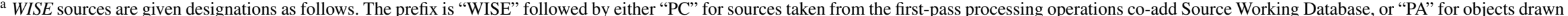

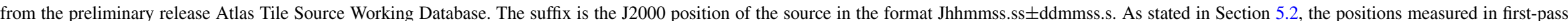
WISE processing and used to derive these designations should not be used for astrometric purposes. Instead, refer to the re-measured astrometry given in Table 6.

${ }^{\mathrm{b}}$ Identified as a high-motion object by Deacon et al. (2005) and classified in the optical as an early-L dwarf by Martín et al. (2010). Alternate name is SIPS J0031-3840.

${ }^{c}$ Identified as a high-motion object by Deacon et al. (2009). Alternate name is ULAS2MASS J0106+1518.

${ }^{\mathrm{d}}$ Identified as a brown dwarf candidate by Zhang et al. (2009). Alternate name is SDSS J131142.11+362923.9.

Discovery Reference. (1) This paper; (2) Mainzer et al. 2011; (3) Burgasser et al. 2011a; (4) Gelino et al. 2011; (5) Cushing et al. 2011. 


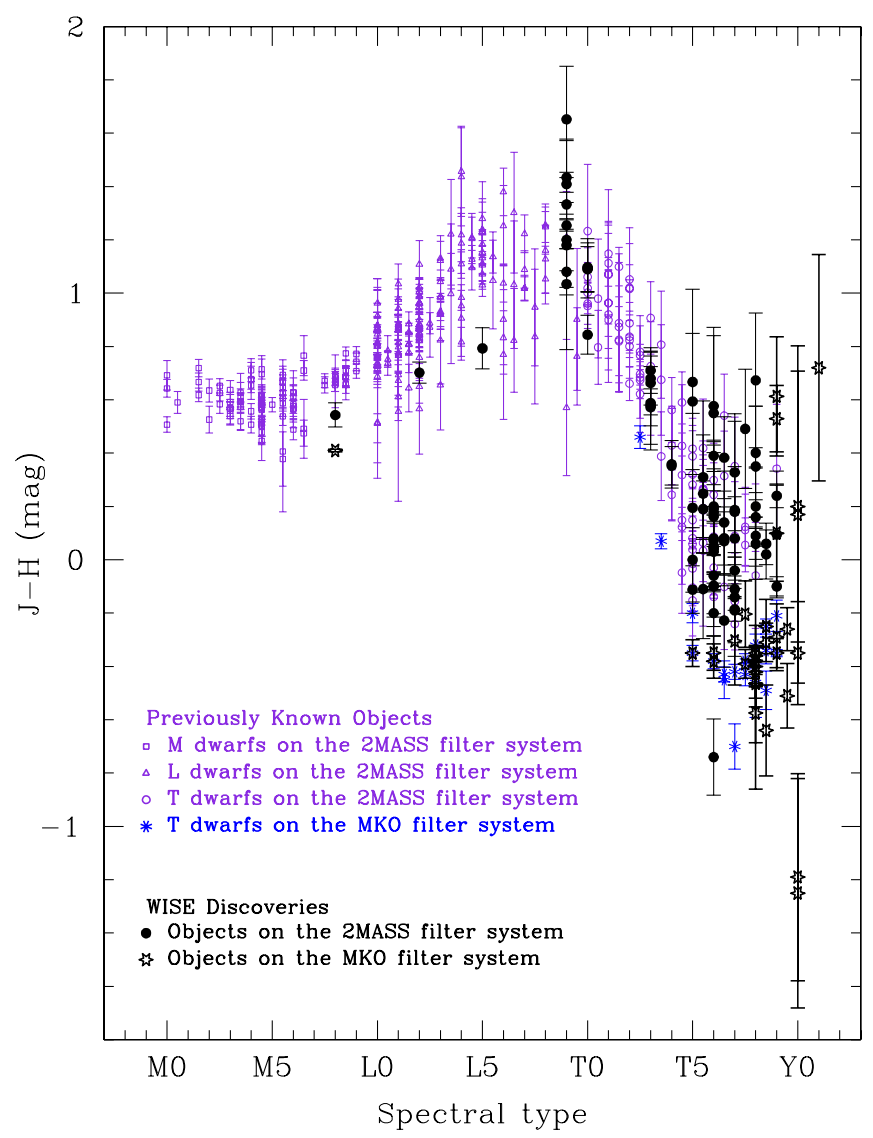

Figure 5. $J-H$ color vs. spectral type for objects with solid $J-H$ colors (not limits). Color coding and symbol selection are explained in the legend.

(A color version of this figure is available in the online journal.)

Kouzuma \& Yamaoka 2010)— that are not brown dwarfs and have $J-H$ colors below 0.4 mag.

Fortunately, other colors like $J-W 2$ and $H-W 2$ can also be used to distinguish populations. Figures 7 and 8 show these colors as a function of spectral type and demonstrate that the $J-W 2$ color of mid- to late-T dwarfs runs from $\sim 2.0$ mag at T5 to $>4.0$ mag at late-T; $H-W 2$ color runs from $\sim 1.5 \mathrm{mag}$ to $\sim 5.0 \mathrm{mag}$ for the same range of types. Figures 9 and 10 show the trend of $J-W 2$ and $H-W 2$ with $W 1-W 2$ color. The correlation is very tight for $\mathrm{M}$ and $\mathrm{L}$ dwarfs $(W 1-W 2<$ $0.6 \mathrm{mag})$, but at redder $W 1-W 2$ colors, corresponding to the $\mathrm{L} / \mathrm{T}$ transition and beyond, there is a much larger spread of $J-W 2$ and $H-W 2$ colors at a given $W 1-W 2$ color. Nonetheless, both $J-W 2$ and $H-W 2$ color increase dramatically beyond $W 1-W 2>1.5 \mathrm{mag}$ ( $>$ T5).

With our list of brown dwarf candidates in hand, we have obtained $J$ and $H$ observations - and in some cases, $Y$ and $K_{s}$ as well-using a variety of different facilities in both hemispheres. Details of those observations are given below, and a listing of the resultant photometry can be found in Table 3 .

\subsubsection{Fan Mountain/FanCam}

The Fan Mountain Near-infrared Camera (FanCam) at the University of Virginia's 31 inch telescope has a $1024 \times$ 1024 pixel HAWAII-1 array (pixel scale of $0.51 \operatorname{arcsec}$ pixel $^{-1}$ ) that images an 8.7 arcmin square field of view (Kanneganti et al. 2009). Observations of eight of our candidates were obtained in the 2MASS $J$ and $H$ bands, and for some objects $Y$-band

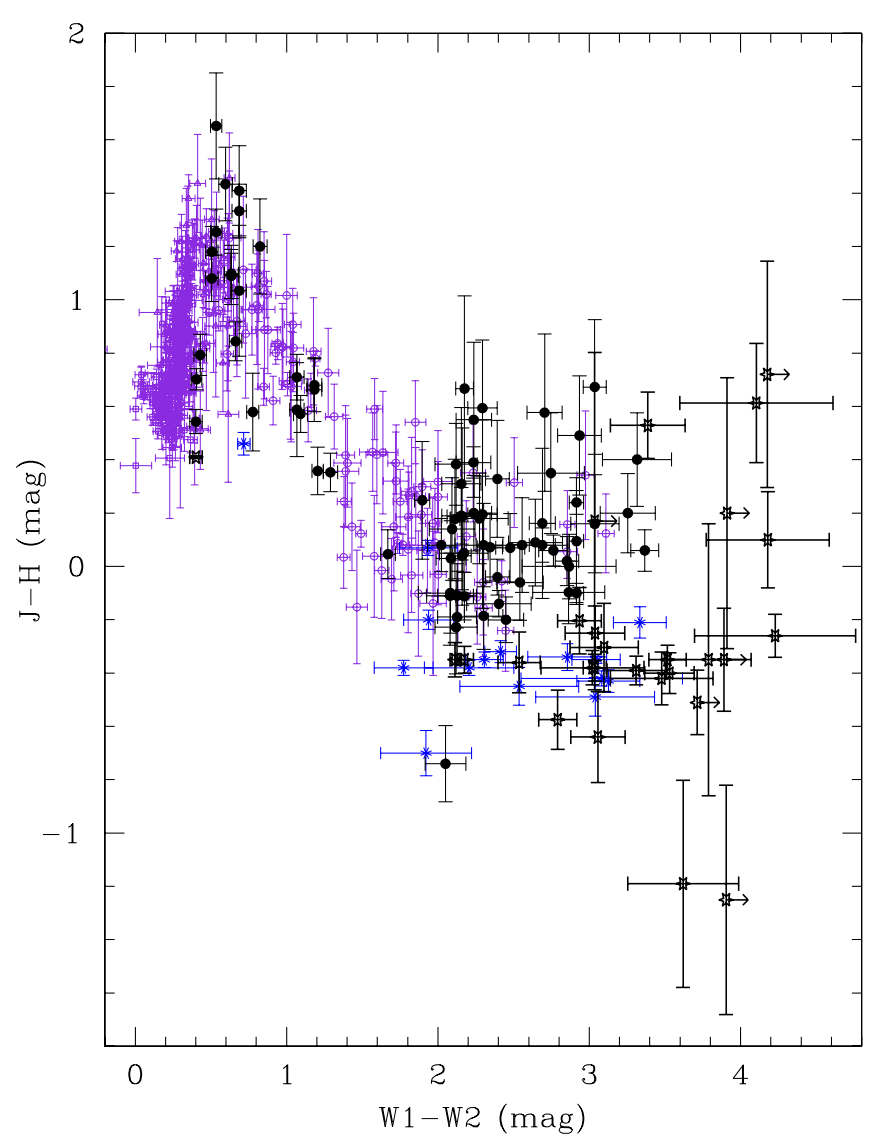

Figure 6. $J-H$ color vs. $W 1-W 2$ color. Symbols are the same as in Figure 5. (A color version of this figure is available in the online journal.)

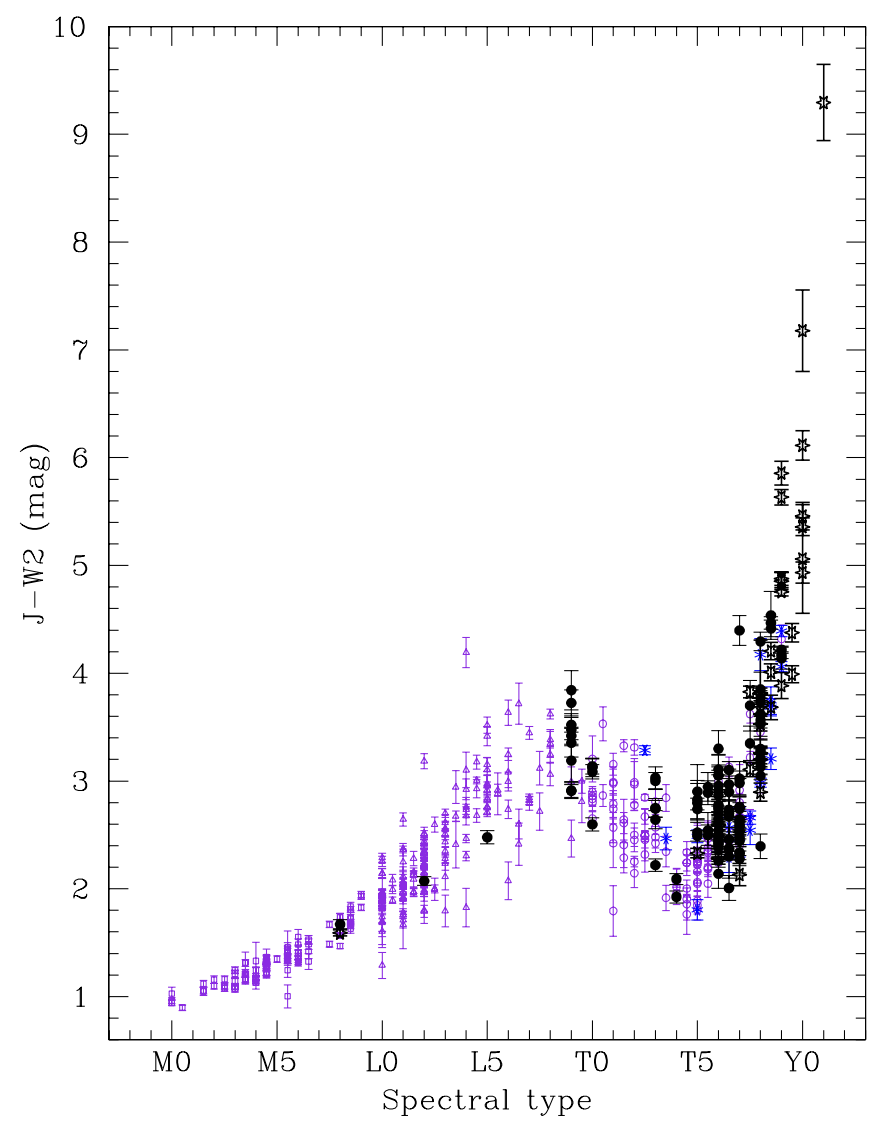

Figure 7. $J-W 2$ color vs. spectral type. Color coding is the same as in Figure 5. (A color version of this figure is available in the online journal.) 
Table 3

Follow-up Photometry of WISE Brown Dwarf Discoveries

\begin{tabular}{|c|c|c|c|c|c|c|c|c|c|c|c|}
\hline \multirow{2}{*}{$\begin{array}{l}\text { Object } \\
\text { Name }\end{array}$} & \multirow{2}{*}{$\begin{array}{l}\text { Obs. } \\
\text { Ref. }\end{array}$} & \multirow{2}{*}{$\begin{array}{c}Y \\
(\mathrm{mag})\end{array}$} & \multicolumn{3}{|c|}{ Two Micron All-Sky Survey Filter System } & \multicolumn{3}{|c|}{ Mauna Kea Observatories Filter System } & \multicolumn{3}{|c|}{ Spitzer/IRAC Observations } \\
\hline & & & $\begin{array}{c}J \\
(\mathrm{mag}) \\
(4)\end{array}$ & $\begin{array}{c}H \\
(\mathrm{mag}) \\
(5)\end{array}$ & $\begin{array}{c}K_{s} \\
(\mathrm{mag}) \\
(6)\end{array}$ & $\begin{array}{c}J \\
(\mathrm{mag}) \\
(7)\end{array}$ & $\begin{array}{c}H \\
(\mathrm{mag}) \\
(8)\end{array}$ & $\begin{array}{c}K_{S} \\
(\mathrm{mag}) \\
(9)\end{array}$ & $\begin{array}{c}c h 1 \\
(\mathrm{mag}) \\
(10)\end{array}$ & $\begin{array}{c}\text { ch2 } \\
\text { (mag) } \\
(11)\end{array}$ & $\begin{array}{c}\text { ch1 } 1-c h 2 \\
(\mathrm{mag}) \\
(12)\end{array}$ \\
\hline WISE J0008-1739 & 4 & $\cdots$ & $17.03 \pm 0.06$ & $17.77 \pm 0.13$ & $>17.0$ & $\cdots$ & $\cdots$ & $\cdots$ & $16.044 \pm 0.030$ & $14.867 \pm 0.021$ & $1.177 \pm 0.037$ \\
\hline WISE J0031-3840 & 1 & $\ldots$ & $14.101 \pm 0.032$ & $13.399 \pm 0.023$ & $12.924 \pm 0.034$ & $\ldots$ & $\ldots$ & $\ldots$ & extant & & \\
\hline \multirow[t]{2}{*}{ WISE J0049+0441 } & 1 & $\ldots$ & $15.854 \pm 0.067$ & $14.674 \pm 0.068$ & $14.170 \pm 0.066$ & $\ldots$ & $\ldots$ & $\ldots$ & $12.977 \pm 0.017$ & $12.950 \pm 0.017$ & $0.027 \pm 0.024$ \\
\hline & 3 & $\ldots$ & $15.85 \pm 0.05$ & $14.77 \pm 0.07$ & $14.17 \pm 0.04$ & $\ldots$ & $\ldots$ & $\ldots$ & & & \\
\hline \multirow[t]{2}{*}{ WISE J0106+1518 } & 1 & $\ldots$ & $14.358 \pm 0.031$ & $13.815 \pm 0.033$ & $13.434 \pm 0.053$ & $\ldots$ & . & $\ldots$ & $12.862 \pm 0.017$ & $12.716 \pm 0.017$ & $0.146 \pm 0.024$ \\
\hline & 10 & $15.095 \pm 0.004$ & $\ldots$ & $\ldots$ & $\ldots$ & $14.277 \pm 0.003$ & $13.867 \pm 0.004$ & $13.363 \pm 0.004$ & . & & \\
\hline WISE J0123+4142 & 3 & $\ldots$ & $17.38 \pm 0.11$ & $17.20 \pm 0.13$ & $18.37 \pm 0.37$ & $\ldots$ & $\ldots$ & $\ldots$ & $16.125 \pm 0.032$ & $14.845 \pm 0.021$ & $1.280 \pm 0.038$ \\
\hline \multirow[t]{2}{*}{ WISE J0138-0322 } & 1 & $\ldots$ & $16.389 \pm 0.096$ & $15.801 \pm 0.147$ & $15.198 \pm 0.129$ & $\ldots$ & $\ldots$ & $\ldots$ & $13.888 \pm 0.018$ & $13.426 \pm 0.018$ & $0.461 \pm 0.026$ \\
\hline & 3 & $\ldots$ & $16.36 \pm 0.07$ & $15.65 \pm 0.05$ & $15.30 \pm 0.08$ & $\ldots$ & & $\ldots$ & & & \\
\hline WISE J0148-7202 & 8 & $\ldots$ & & & & $18.96 \pm 0.07$ & $19.22 \pm 0.04$ & $\ldots$ & $16.844 \pm 0.045$ & $14.650 \pm 0.020$ & $2.194 \pm 0.050$ \\
\hline \multirow[t]{3}{*}{ WISE J0150+3827 } & 1 & $\ldots$ & $16.111 \pm 0.077$ & $15.018 \pm 0.080$ & $14.477 \pm 0.070$ & $\ldots$ & $\ldots$ & $\ldots$ & $13.236 \pm 0.017$ & $13.117 \pm 0.017$ & $0.119 \pm 0.025$ \\
\hline & 5 & $\ldots$ & $16.12 \pm 0.06$ & $15.03 \pm 0.06$ & & $\ldots$ & $\ldots$ & $\ldots$ & $\ldots$ & .. & $\ldots$ \\
\hline & 4 & $\ldots$ & $16.070 \pm 0.054$ & $14.973 \pm 0.074$ & $14.376 \pm 0.067$ & $\ldots$ & $\ldots$ & $\ldots$ & $\ldots$ & . & $\ldots$ \\
\hline WISE J0206+2640 & 1 & $\ldots$ & $16.530 \pm 0.114$ & $15.096 \pm 0.077$ & $14.523 \pm 0.076$ & $\ldots$ & $\ldots$ & $\ldots$ & $13.075 \pm 0.017$ & $12.867 \pm 0.017$ & $0.208 \pm 0.024$ \\
\hline WISE J0221+3842 & 3 & $\ldots$ & $17.59 \pm 0.12$ & $17.45 \pm 0.15$ & $16.95 \pm 0.17$ & & & $\ldots$ & $15.931 \pm 0.029$ & $14.863 \pm 0.021$ & $1.068 \pm 0.036$ \\
\hline WISE J0223-2932 & 4 & $\ldots$ & $17.341 \pm 0.153$ & $16.850 \pm 0.163$ & $>16.94$ & $17.100 \pm 0.050$ & $17.304 \pm 0.114$ & $\ldots$ & $15.810 \pm 0.028$ & $14.015 \pm 0.019$ & $1.795 \pm 0.033$ \\
\hline WISE J0226-0211 & 5 & $\ldots$ & $18.94 \pm 0.12$ & $>19.10$ & & $\ldots$ & $\ldots$ & $\ldots$ & $16.599 \pm 0.040$ & $14.675 \pm 0.021$ & $1.924 \pm 0.045$ \\
\hline \multirow[t]{2}{*}{ WISE J0254+0223 } & 1 & $\ldots$ & $16.557 \pm 0.156$ & $15.884 \pm 0.199$ & $>16.006$ & $\ldots$ & $\ldots$ & $\ldots$ & $14.692 \pm 0.021$ & $12.710 \pm 0.017$ & $1.982 \pm 0.027$ \\
\hline & 7 & $\ldots$ & & & $\ldots$ & $15.91 \pm 0.03$ & $16.29 \pm 0.04$ & $\ldots$ & $\cdots$ & & \\
\hline WISE J0305+3954 & 3 & $\ldots$ & $17.13 \pm 0.09$ & $17.08 \pm 0.12$ & $16.93 \pm 0.17$ & $\ldots$ & $\ldots$ & $\ldots$ & $15.981 \pm 0.030$ & $14.542 \pm 0.020$ & $1.440 \pm 0.036$ \\
\hline \multirow[t]{2}{*}{ WISE J0307+2904 } & 3 & $\ldots$ & $17.78 \pm 0.11$ & $17.70 \pm 0.14$ & $17.78 \pm 0.21$ & $\ldots$ & $\ldots$ & $\ldots$ & $16.385 \pm 0.036$ & $14.967 \pm 0.022$ & $1.419 \pm 0.043$ \\
\hline & 10 & $\ldots$ & & & $\ldots$ & $\ldots$ & $\ldots$ & $18.084 \pm 0.122$ & & & \\
\hline WISE J0313+7807 & 6 & $18.27 \pm 0.05$ & $17.65 \pm 0.07$ & $17.63 \pm 0.06$ & $\ldots$ & $\ldots$ & 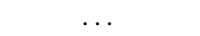 & $\ldots$ & $15.310 \pm 0.024$ & $13.268 \pm 0.017$ & $2.042 \pm 0.029$ \\
\hline WISE J0323-6025 & 8 & $\ldots$ & & & $\cdots$ & $18.15 \pm 0.10$ & $18.40 \pm 0.02$ & $\ldots$ & $16.572 \pm 0.039$ & $14.506 \pm 0.020$ & $2.066 \pm 0.043$ \\
\hline WISE J0333-5856 & 1 & $\ldots$ & $15.997 \pm 0.083$ & $15.418 \pm 0.120$ & $14.639 \pm 0.097$ & $\ldots$ & $\ldots$ & $\ldots$ & $13.590 \pm 0.018$ & $13.297 \pm 0.017$ & $0.293 \pm 0.025$ \\
\hline WISE J0410+1502 & 7 & $\ldots$ & $\ldots$ & $\ldots$ & $\ldots$ & $19.25 \pm 0.5$ & $19.05 \pm 0.09$ & $\ldots$ & $16.642 \pm 0.042$ & $14.183 \pm 0.019$ & $2.459 \pm 0.046$ \\
\hline \multirow{2}{*}{ WISE J0410+1411 } & 3 & $\ldots$ & $17.16 \pm 0.09$ & $17.26 \pm 0.12$ & $16.98 \pm 0.18$ & $\ldots$ & $\ldots$ & $\ldots$ & $16.099 \pm 0.032$ & $15.001 \pm 0.022$ & $1.098 \pm 0.039$ \\
\hline & 10 & $\cdots$ & $\cdots$ & $\ldots$ & $\cdots$ & $\cdots$ & $\cdots$ & $17.823 \pm 0.196$ & $\cdots$ & $\cdots$ & $\cdots$ \\
\hline \multirow[t]{2}{*}{ WISE J0448-1935 } & 2 & $\ldots$ & $17.016 \pm 0.172$ & $16.821 \pm 0.309$ & $>15.858$ & $\ldots$ & $\ldots$ & $\ldots$ & $\ldots$ & .. & $\ldots$ \\
\hline & 2 & $\ldots$ & $16.986 \pm 0.173$ & $16.392 \pm 0.188$ & $>16.556$ & $\ldots$ & $\ldots$ & $\ldots$ & $\ldots$ & . & $\ldots$ \\
\hline WISE J0458+6434 & 6 & $18.34 \pm 0.07$ & $17.47 \pm 0.05$ & $17.41 \pm 0.06$ & $\ldots$ & $\ldots$ & $\ldots$ & $\ldots$ & $15.080 \pm 0.022$ & $12.985 \pm 0.017$ & $2.094 \pm 0.028$ \\
\hline WISE J0500-1223 & 9 & $\ldots$ & $\ldots$ & $\ldots$ & $\ldots$ & $17.782 \pm 0.496$ & $18.132 \pm 0.121$ & $\ldots$ & $15.947 \pm 0.029$ & $13.999 \pm 0.019$ & $1.948 \pm 0.035$ \\
\hline \multirow[t]{2}{*}{ WISE J0513+0608 } & 1 & $\ldots$ & $16.205 \pm 0.094$ & $>16.890$ & $>15.936$ & $\ldots$ & $\ldots$ & $\ldots$ & $15.108 \pm 0.022$ & $13.949 \pm 0.018$ & $1.160 \pm 0.029$ \\
\hline & 3 & $\ldots$ & $16.21 \pm 0.06$ & $16.13 \pm 0.08$ & $16.05 \pm 0.11$ & $\ldots$ & $\ldots$ & $\ldots$ & $\ldots$ & & \\
\hline WISE J0525+6739 & 7 & $\ldots$ & $\ldots$ & $\ldots$ & $\ldots$ & $17.49 \pm 0.04$ & $17.87 \pm 0.05$ & & $16.404 \pm 0.036$ & $14.881 \pm 0.021$ & $1.522 \pm 0.042$ \\
\hline WISE J0528-3308 & 9 & $\ldots$ & & & & $16.666 \pm 0.087$ & $16.970 \pm 0.141$ & $17.119 \pm 0.153$ & $16.308 \pm 0.034$ & $14.593 \pm 0.020$ & $1.716 \pm 0.040$ \\
\hline WISE J0539-1034 & 3 & $\ldots$ & $17.71 \pm 0.11$ & $17.82 \pm 0.15$ & $18.40 \pm 0.31$ & $\ldots$ & $\ldots$ & $\ldots$ & $16.145 \pm 0.032$ & $15.008 \pm 0.021$ & $1.137 \pm 0.038$ \\
\hline \multirow[t]{2}{*}{ WISE J0542-1628 } & 1 & $\ldots$ & $16.577 \pm 0.135$ & $>15.887$ & $>15.934$ & $\ldots$ & $\ldots$ & $\ldots$ & $15.265 \pm 0.023$ & $13.968 \pm 0.018$ & $1.297 \pm 0.030$ \\
\hline & 3 & $\ldots$ & $16.64 \pm 0.08$ & $16.57 \pm 0.10$ & $16.63 \pm 0.14$ & $\ldots$ & $\ldots$ & $\ldots$ & $\ldots$ & & \\
\hline WISE J0611-0410 & 1 & $\ldots$ & $15.489 \pm 0.055$ & $14.645 \pm 0.048$ & $14.221 \pm 0.070$ & $\ldots$ & $\ldots$ & $\ldots$ & $13.069 \pm 0.017$ & $12.924 \pm 0.017$ & $0.145 \pm 0.024$ \\
\hline \multirow{2}{*}{ WISE J0612-3036 } & 2 & $\ldots$ & $17.096 \pm 0.191$ & $>15.808$ & $>15.781$ & $\ldots$ & $\ldots$ & $\ldots$ & $15.588 \pm 0.026$ & $14.033 \pm 0.019$ & $1.555 \pm 0.032$ \\
\hline & 3 & $\ldots$ & $17.00 \pm 0.09$ & $17.06 \pm 0.11$ & $17.34 \pm 0.21$ & $\ldots$ & $\ldots$ & $\ldots$ & .. & & \\
\hline WISE J0612-4920 & $\ldots$ & $\ldots$ & $\ldots$ & $\ldots$ & $\ldots$ & $\ldots$ & $\ldots$ & $\ldots$ & $14.732 \pm 0.021$ & $14.130 \pm 0.019$ & $0.602 \pm 0.028$ \\
\hline
\end{tabular}


Table 3

(Continued)

\begin{tabular}{|c|c|c|c|c|c|c|c|c|c|c|c|}
\hline \multirow{3}{*}{$\begin{array}{l}\text { Object } \\
\text { Name } \\
\text { (1) }\end{array}$} & \multirow{3}{*}{$\begin{array}{l}\text { Obs. } \\
\text { Ref. } \\
\text { (2) }\end{array}$} & \multirow{3}{*}{$\begin{array}{c}Y \\
(\mathrm{mag}) \\
(3)\end{array}$} & \multicolumn{3}{|c|}{ Two Micron All-Sky Survey Filter System } & \multicolumn{3}{|c|}{ Mauna Kea Observatories Filter System } & \multicolumn{3}{|c|}{ Spitzer/IRAC Observations } \\
\hline & & & $\begin{array}{c}J \\
(\mathrm{mag})\end{array}$ & $\begin{array}{c}H \\
(\mathrm{mag})\end{array}$ & $\begin{array}{c}K_{S} \\
(\mathrm{mag})\end{array}$ & $\begin{array}{c}J \\
(\mathrm{mag})\end{array}$ & $\begin{array}{c}H \\
(\mathrm{mag})\end{array}$ & $\begin{array}{c}K_{s} \\
(\mathrm{mag})\end{array}$ & $\begin{array}{c}\text { ch } 1 \\
(\mathrm{mag})\end{array}$ & $\begin{array}{c}\text { ch2 } \\
(\mathrm{mag})\end{array}$ & $\begin{array}{c}\operatorname{ch} 1-\operatorname{ch} 2 \\
(\mathrm{mag})\end{array}$ \\
\hline & & & (4) & $(5)$ & $(6)$ & $(7)$ & $(8)$ & (9) & $(10)$ & $(11)$ & (12) \\
\hline WISE J0614+3912 & 2 & $\ldots$ & $16.933 \pm 0.163$ & $16.356 \pm 0.246$ & $>16.283$ & $\ldots$ & $\ldots$ & $\ldots$ & $15.190 \pm 0.023$ & $13.599 \pm 0.018$ & $1.591 \pm 0.029$ \\
\hline WISE J0623-0456 & 3 & $\ldots$ & $17.51 \pm 0.10$ & $17.31 \pm 0.11$ & $17.80 \pm 0.22$ & $\ldots$ & $\ldots$ & $\ldots$ & $15.494 \pm 0.025$ & $13.736 \pm 0.018$ & $1.759 \pm 0.031$ \\
\hline \multirow[t]{2}{*}{ WISE J0625+5646 } & 1 & $\ldots$ & $16.783 \pm 0.151$ & $16.233 \pm 0.248$ & $>15.771$ & $\ldots$ & $\ldots$ & $\ldots$ & $15.470 \pm 0.025$ & $14.414 \pm 0.019$ & $1.056 \pm 0.031$ \\
\hline & 3 & $\ldots$ & $17.10 \pm 0.10$ & $16.90 \pm 0.10$ & $16.84 \pm 0.15$ & $\ldots$ & $\ldots$ & $\ldots$ & ... & $\ldots$ & $\ldots$ \\
\hline WISE J0627-1114 & 1 & $\ldots$ & $15.487 \pm 0.052$ & $15.441 \pm 0.075$ & $15.432 \pm 0.181$ & $\ldots$ & $\ldots$ & $\ldots$ & $14.272 \pm 0.019$ & $13.326 \pm 0.018$ & $0.946 \pm 0.026$ \\
\hline WISE J0656+4205 & 1 & $\ldots$ & $15.446 \pm 0.049$ & $14.874 \pm 0.049$ & $14.831 \pm 0.096$ & $\ldots$ & $\ldots$ & $\ldots$ & $\ldots$ & $\ldots$ & $\ldots$ \\
\hline WISE J0744+5628 & 3 & $\ldots$ & $17.68 \pm 0.11$ & $17.59 \pm 0.12$ & $17.57 \pm 0.20$ & $\ldots$ & $\ldots$ & $\ldots$ & $\ldots$ & $\ldots$ & $\ldots$ \\
\hline \multirow[t]{4}{*}{ WISE J0750+2725 } & 4 & $\ldots$ & $>18.57$ & $>17.92$ & $>16.91$ & $\ldots$ & $\ldots$ & $\ldots$ & $16.679 \pm 0.042$ & $14.486 \pm 0.020$ & $2.194 \pm 0.046$ \\
\hline & 3 & $\ldots$ & $19.02 \pm 0.21$ & $>19.49$ & $>18.63$ & $\ldots$ & $\ldots$ & $\ldots$ & $\ldots$ & $\ldots$ & $\ldots$ \\
\hline & 7 & $\ldots$ & $\ldots$ & $\ldots$ & $\ldots$ & $18.69 \pm 0.04$ & $19.00 \pm 0.06$ & $\ldots$ & $\ldots$ & $\ldots$ & $\ldots$ \\
\hline & 10 & $\ldots$ & $\ldots$ & $\ldots$ & $\ldots$ & $18.744 \pm 0.053$ & $\ldots$ & $\ldots$ & $\ldots$ & $\ldots$ & $\ldots$ \\
\hline WISE J0751-7634 & 9 & $\ldots$ & $\ldots$ & $\ldots$ & $\ldots$ & $19.342 \pm 0.048$ & $>19.02$ & $\ldots$ & $16.432 \pm 0.036$ & $14.621 \pm 0.020$ & $1.811 \pm 0.041$ \\
\hline WISE J0759-4904 & $\ldots$ & $\ldots$ & $\ldots$ & $\ldots$ & $\ldots$ & $\ldots$ & $\ldots$ & $\ldots$ & $15.623 \pm 0.026$ & $13.761 \pm 0.018$ & $1.862 \pm 0.032$ \\
\hline WISE J0819-0335 & 1 & $\ldots$ & $14.991 \pm 0.044$ & $14.638 \pm 0.057$ & $14.586 \pm 0.105$ & $\ldots$ & $\ldots$ & $\ldots$ & $13.608 \pm 0.018$ & $13.071 \pm 0.017$ & $0.537 \pm 0.025$ \\
\hline \multirow[t]{2}{*}{ WISE J0821+1443 } & 1 & $\ldots$ & $16.825 \pm 0.155$ & $16.515 \pm 0.240$ & $>17.089$ & $\ldots$ & $\ldots$ & $\ldots$ & $\ldots$ & $\ldots$ & $\ldots$ \\
\hline & 3 & $\ldots$ & $16.78 \pm 0.07$ & $16.59 \pm 0.08$ & $16.58 \pm 0.12$ & $\ldots$ & $\ldots$ & $\ldots$ & $\ldots$ & $\ldots$ & $\ldots$ \\
\hline WISE J0836-1859 & $\ldots$ & $\ldots$ & $\ldots$ & $\ldots$ & $\ldots$ & $\ldots$ & $\ldots$ & $\ldots$ & $16.884 \pm 0.047$ & $15.091 \pm 0.022$ & $1.793 \pm 0.052$ \\
\hline WISE J0857+5604 & 3 & $\ldots$ & $17.65 \pm 0.12$ & $17.49 \pm 0.14$ & $17.69 \pm 0.22$ & $\ldots$ & $\ldots$ & $\ldots$ & $16.026 \pm 0.030$ & $14.137 \pm 0.019$ & $1.889 \pm 0.035$ \\
\hline \multirow[t]{2}{*}{ WISE J0906+4735 } & 4 & $\ldots$ & $18.126 \pm 0.134$ & $>18.33$ & $>17.36$ & $\ldots$ & $\ldots$ & $\ldots$ & $16.474 \pm 0.037$ & $14.554 \pm 0.020$ & $1.920 \pm 0.042$ \\
\hline & 3 & $\ldots$ & $18.16 \pm 0.16$ & $17.81 \pm 0.16$ & $18.54 \pm 0.34$ & $\ldots$ & $\ldots$ & $\ldots$ & $\ldots$ & $\ldots$ & $\ldots$ \\
\hline \multirow[t]{2}{*}{ WISE J0929+0409 } & 4 & $\ldots$ & $17.214 \pm 0.056$ & $>18.26$ & $>17.37$ & $\ldots$ & $\ldots$ & $\ldots$ & $15.717 \pm 0.027$ & $14.238 \pm 0.019$ & $1.479 \pm 0.033$ \\
\hline & 10 & $\ldots$ & $\ldots$ & $\ldots$ & $\ldots$ & $\ldots$ & $17.373 \pm 0.067$ & $17.395 \pm 0.087$ & $\ldots$ & $\ldots$ & $\ldots$ \\
\hline \multirow[t]{2}{*}{ WISE J0952+1955 } & 2 & $\ldots$ & $17.293 \pm 0.179$ & $>17.166$ & $>16.447$ & $\ldots$ & $\ldots$ & $\ldots$ & $15.815 \pm 0.028$ & $14.499 \pm 0.020$ & $1.316 \pm 0.035$ \\
\hline & 4 & $\ldots$ & $17.126 \pm 0.062$ & $17.223 \pm 0.100$ & $>17.337$ & $\ldots$ & $\ldots$ & $\ldots$ & $\ldots$ & $\ldots$ & $\ldots$ \\
\hline WISE J1018-2445 & $\ldots$ & $\ldots$ & $\ldots$ & $\ldots$ & $\ldots$ & $\ldots$ & $\ldots$ & $\ldots$ & $16.132 \pm 0.032$ & $14.137 \pm 0.019$ & $1.994 \pm 0.037$ \\
\hline \multirow[t]{2}{*}{ WISE J1019+6529 } & 1 & $\ldots$ & $16.554 \pm 0.149$ & $>16.328$ & $>17.016$ & $\ldots$ & $\ldots$ & $\ldots$ & $15.313 \pm 0.024$ & $14.004 \pm 0.019$ & $1.309 \pm 0.030$ \\
\hline & 4 & $\ldots$ & $16.589 \pm 0.055$ & $16.517 \pm 0.115$ & $16.457 \pm 0.179$ & $\ldots$ & $\ldots$ & $\ldots$ & $\ldots$ & $\ldots$ & $\ldots$ \\
\hline WISE J1042-3842 & $\ldots$ & $\ldots$ & $\ldots$ & $\ldots$ & $\ldots$ & $\ldots$ & $\ldots$ & $\ldots$ & $16.771 \pm 0.043$ & $14.572 \pm 0.020$ & $2.198 \pm 0.048$ \\
\hline \multirow[t]{2}{*}{ WISE J1122+2550 } & 1 & $\ldots$ & $16.376 \pm 0.130$ & $>16.062$ & $>16.824$ & $\ldots$ & $\ldots$ & $\ldots$ & $15.374 \pm 0.024$ & $14.062 \pm 0.019$ & $1.312 \pm 0.031$ \\
\hline & 3 & $\ldots$ & $16.67 \pm 0.09$ & $16.64 \pm 0.11$ & $16.55 \pm 0.12$ & $\ldots$ & $\ldots$ & $\ldots$ & $\ldots$ & & $\ldots$ \\
\hline WISE J1150+6302 & 4 & $\ldots$ & $17.72 \pm 0.08$ & $>18.01$ & $>16.65$ & $\ldots$ & $\ldots$ & $\ldots$ & $15.614 \pm 0.026$ & $13.429 \pm 0.018$ & $2.185 \pm 0.031$ \\
\hline \multirow[t]{2}{*}{ WISE J1217+1626 } & 4 & $\ldots$ & $>18.52$ & $>17.50$ & $>16.64$ & $\ldots$ & $\ldots$ & $\ldots$ & $15.437 \pm 0.024$ & $13.105 \pm 0.017$ & $2.332 \pm 0.030$ \\
\hline & 7 & $\ldots$ & $\ldots$ & $\ldots$ & $\ldots$ & $17.83 \pm 0.02$ & $18.18 \pm 0.05$ & $\ldots$ & $\ldots$ & & $\ldots$ \\
\hline WISE J1311+0122 & 7 & $\ldots$ & $\ldots$ & $\ldots$ & $\ldots$ & $19.16 \pm 0.12$ & $\ldots$ & $\ldots$ & $16.817 \pm 0.045$ & $14.676 \pm 0.020$ & $2.142 \pm 0.049$ \\
\hline WISE J1311+3629 & 1 & $\ldots$ & $15.545 \pm 0.053$ & $14.752 \pm 0.056$ & $14.140 \pm 0.049$ & $\ldots$ & $\ldots$ & $\ldots$ & $13.164 \pm 0.017$ & $13.191 \pm 0.017$ & $-0.027 \pm 0.025$ \\
\hline \multirow[t]{4}{*}{ WISE J1320+6034 } & 2 & $\ldots$ & $16.789 \pm 0.188$ & $>16.806$ & $>15.622$ & $\ldots$ & $\ldots$ & $\ldots$ & $15.833 \pm 0.028$ & $14.496 \pm 0.020$ & $1.336 \pm 0.034$ \\
\hline & 2 & $\ldots$ & $17.189 \pm 0.257$ & $>15.759$ & $>16.921$ & $\ldots$ & $\ldots$ & $\ldots$ & $\ldots$ & $\ldots$ & $\ldots$ \\
\hline & 4 & $\ldots$ & $16.496 \pm 0.092$ & $16.724 \pm 0.149$ & $16.396 \pm 0.213$ & $\ldots$ & $\ldots$ & $\ldots$ & $\ldots$ & $\ldots$ & $\ldots$ \\
\hline & 4 & $\ldots$ & $16.947 \pm 0.084$ & $16.564 \pm 0.128$ & $17.431 \pm 0.427$ & $\ldots$ & $\ldots$ & $\ldots$ & $\ldots$ & $\ldots$ & $\ldots$ \\
\hline WISE J1322-2340 & 4 & $\ldots$ & $17.006 \pm 0.105$ & $16.605 \pm 0.141$ & $16.991 \pm 0.399$ & $\ldots$ & $\ldots$ & $\ldots$ & $15.666 \pm 0.026$ & $13.892 \pm 0.018$ & $1.775 \pm 0.032$ \\
\hline \multirow[t]{3}{*}{ WISE J1348+6603 } & 1 & $\ldots$ & $16.943 \pm 0.191$ & $15.909 \pm 0.155$ & $15.259 \pm 0.152$ & $\ldots$ & $\ldots$ & $\ldots$ & $13.953 \pm 0.018$ & $13.821 \pm 0.018$ & $0.131 \pm 0.026$ \\
\hline & 4 & $\ldots$ & $17.109 \pm 0.128$ & $15.700 \pm 0.110$ & $15.052 \pm 0.126$ & $\ldots$ & $\ldots$ & $\ldots$ & $\ldots$ & $\ldots$ & $\ldots$ \\
\hline & 4 & $\ldots$ & $17.174 \pm 0.070$ & $15.841 \pm 0.072$ & $15.551 \pm 0.109$ & $\ldots$ & $\ldots$ & $\ldots$ & $\cdots$ & $\ldots$ & $\ldots$ \\
\hline
\end{tabular}


Table 3

Continued)

\begin{tabular}{|c|c|c|c|c|c|c|c|c|c|c|c|}
\hline \multirow{2}{*}{$\begin{array}{l}\text { Object } \\
\text { Name }\end{array}$} & \multirow{2}{*}{$\begin{array}{l}\text { Obs. } \\
\text { Ref. }\end{array}$} & \multirow{2}{*}{$\begin{array}{c}Y \\
(\mathrm{mag})\end{array}$} & \multicolumn{3}{|c|}{ Two Micron All-Sky Survey Filter System } & \multicolumn{3}{|c|}{ Mauna Kea Observatories Filter System } & \multicolumn{3}{|c|}{ Spitzer/IRAC Observations } \\
\hline & & & $\begin{array}{c}J \\
(\mathrm{mag})\end{array}$ & $\begin{array}{c}H \\
(\mathrm{mag})\end{array}$ & $\begin{array}{c}K_{S} \\
(\mathrm{mag})\end{array}$ & $\begin{array}{c}J \\
(\mathrm{mag})\end{array}$ & $\begin{array}{c}H \\
(\mathrm{mag})\end{array}$ & $\begin{array}{c}K_{s} \\
(\mathrm{mag})\end{array}$ & $\begin{array}{c}c h 1 \\
(\mathrm{mag})\end{array}$ & $\begin{array}{c}\text { ch2 } \\
\text { (mag) }\end{array}$ & $\begin{array}{c}\text { ch } 1-\operatorname{ch} 2 \\
(\mathrm{mag})\end{array}$ \\
\hline (1) & $(2)$ & (3) & (4) & $(5)$ & (6) & (7) & (8) & (9) & $(10)$ & (11) & (12) \\
\hline WISE J1405+5534 & 7 & $\ldots$ & $\ldots$ & $\ldots$ & $\ldots$ & $20.20 \pm 0.13$ & $21.45 \pm 0.41$ & $\ldots$ & $16.884 \pm 0.047$ & $14.061 \pm 0.019$ & $2.823 \pm 0.050$ \\
\hline WISE J1436-1814 & $\ldots$ & $\ldots$ & $\ldots$ & $\ldots$ & $\ldots$ & $\ldots$ & $\ldots$ & $\ldots$ & $15.990 \pm 0.030$ & $14.718 \pm 0.021$ & $1.272 \pm 0.036$ \\
\hline \multirow[t]{4}{*}{ WISE J1457+5815 } & 2 & $\ldots$ & $17.137 \pm 0.261$ & $>17.636$ & $>17.320$ & $\ldots$ & $\ldots$ & $\ldots$ & $15.853 \pm 0.028$ & $14.443 \pm 0.019$ & $1.411 \pm 0.034$ \\
\hline & 2 & $\ldots$ & $16.954 \pm 0.218$ & $>16.725$ & $>15.464$ & $\ldots$ & $\ldots$ & $\ldots$ & $\ldots$ & $\ldots$ & $\ldots$ \\
\hline & 2 & $\ldots$ & $16.825 \pm 0.169$ & $16.638 \pm 0.287$ & $>16.704$ & $\ldots$ & $\ldots$ & $\ldots$ & $\ldots$ & $\ldots$ & $\ldots$ \\
\hline & 4 & $\ldots$ & $>17.48$ & $>16.42$ & $>15.03$ & $\ldots$ & $\ldots$ & $\ldots$ & $\ldots$ & $\ldots$ & $\ldots$ \\
\hline \multirow[t]{2}{*}{ WISE J1506+7027 } & 7 & $\ldots$ & $\ldots$ & $\ldots$ & $\ldots$ & $13.56 \pm 0.05$ & $13.91 \pm 0.04$ & $\ldots$ & $12.629 \pm 0.017$ & $11.315 \pm 0.016$ & $1.314 \pm 0.023$ \\
\hline & 4 & $\ldots$ & $14.328 \pm 0.095$ & $14.150 \pm 0.203$ & $14.048 \pm 0.136$ & $\ldots$ & $\ldots$ & $\ldots$ & $\ldots$ & .. & $\ldots$ \\
\hline \multirow[t]{2}{*}{ WISE J1519+7009 } & 4 & $\ldots$ & $>17.58$ & $>16.82$ & $\ldots$ & $\ldots$ & $\ldots$ & $\ldots$ & $16.194 \pm 0.033$ & $14.088 \pm 0.019$ & $2.105 \pm 0.038$ \\
\hline & 7 & $\ldots$ & $\ldots$ & $\ldots$ & $\ldots$ & $17.88 \pm 0.03$ & $18.28 \pm 0.07$ & $\ldots$ & & & \\
\hline WISE J1541-2250 & 12 & $\ldots$ & $\ldots$ & $\ldots$ & $\ldots$ & $21.16 \pm 0.36$ & $20.99 \pm 0.52$ & $\ldots$ & $16.725 \pm 0.044$ & $14.230 \pm 0.020$ & $2.495 \pm 0.048$ \\
\hline WISE J1612-3420 & $\ldots$ & $\ldots$ & $\ldots$ & $\ldots$ & $\ldots$ & $\ldots$ & $\ldots$ & $\ldots$ & $15.445 \pm 0.025$ & $13.856 \pm 0.018$ & $1.589 \pm 0.031$ \\
\hline \multirow[t]{2}{*}{ WISE J1614+1739 } & 6 & $19.43 \pm 0.10$ & $\ldots$ & $\ldots$ & $\ldots$ & $\ldots$ & $\ldots$ & $\ldots$ & $16.426 \pm 0.036$ & $14.218 \pm 0.019$ & $2.209 \pm 0.041$ \\
\hline & 9 & $\ldots$ & $\ldots$ & $\ldots$ & $\ldots$ & $19.084 \pm 0.059$ & $18.471 \pm 0.216$ & $\ldots$ & $\ldots$ & & $\ldots$ \\
\hline \multirow[t]{2}{*}{ WISE J1617+1807 } & 6 & $18.71 \pm 0.04$ & $\ldots$ & $\ldots$ & $\ldots$ & $\ldots$ & $\ldots$ & $\ldots$ & $15.964 \pm 0.029$ & $14.097 \pm 0.019$ & $1.868 \pm 0.035$ \\
\hline & 9 & $\ldots$ & $\ldots$ & $\ldots$ & $\ldots$ & $17.659 \pm 0.080$ & $18.234 \pm 0.078$ & $\ldots$ & $\ldots$ & $\ldots$ & $\ldots$ \\
\hline \multirow[t]{2}{*}{ WISE J1622-0959 } & 1 & $\ldots$ & $16.398 \pm 0.118$ & $>16.615$ & $15.737 \pm 0.257$ & $\ldots$ & $\ldots$ & $\ldots$ & $15.363 \pm 0.024$ & $14.146 \pm 0.019$ & $1.217 \pm 0.031$ \\
\hline & 4 & $\ldots$ & $16.44 \pm 0.03$ & $16.05 \pm 0.05$ & $16.07 \pm 0.11$ & $\ldots$ & $\ldots$ & $\ldots$ & $\ldots$ & $\cdots$ & $\ldots$ \\
\hline \multirow[t]{3}{*}{ WISE J1627+3255 } & 1 & $\ldots$ & $16.720 \pm 0.128$ & $16.558 \pm 0.251$ & $>17.362$ & $\ldots$ & $\ldots$ & $\ldots$ & $15.219 \pm 0.023$ & $13.618 \pm 0.018$ & $1.601 \pm 0.029$ \\
\hline & 4 & $\ldots$ & $16.48 \pm 0.04$ & $16.40 \pm 0.05$ & $\ldots$ & $\ldots$ & $\ldots$ & $\ldots$ & $\ldots$ & $\ldots$ & $\ldots$ \\
\hline & 6 & $17.49 \pm 0.02$ & $16.61 \pm 0.02$ & & $\ldots$ & $\ldots$ & $\ldots$ & $\ldots$ & $\ldots$ & $\ldots$ & $\ldots$ \\
\hline \multirow[t]{2}{*}{ WISE J1647+5632 } & 1 & $\ldots$ & $16.911 \pm 0.180$ & $15.259 \pm 0.084$ & $14.611 \pm 0.087$ & $\ldots$ & $\ldots$ & $\ldots$ & $13.253 \pm 0.017$ & $13.128 \pm 0.017$ & $0.125 \pm 0.025$ \\
\hline & 4 & $\ldots$ & $16.590 \pm 0.062$ & $15.336 \pm 0.060$ & $14.483 \pm 0.072$ & $\ldots$ & $\ldots$ & $\ldots$ & $\ldots$ & & .. \\
\hline WISE J1653+4444 & 6 & $18.11 \pm 0.02$ & $17.59 \pm 0.03$ & $17.53 \pm 0.05$ & $\ldots$ & $\ldots$ & $\ldots$ & $\ldots$ & $15.671 \pm 0.026$ & $13.869 \pm 0.018$ & $1.802 \pm 0.032$ \\
\hline WISE J1711+3500 & 4 & $\ldots$ & $17.886 \pm 0.130$ & $>18.12$ & $\ldots$ & $\ldots$ & $\ldots$ & $\ldots$ & $16.459 \pm 0.037$ & $14.623 \pm 0.020$ & $1.836 \pm 0.042$ \\
\hline \multirow[t]{2}{*}{ WISE J1717+6129 } & 7 & $\ldots$ & $\ldots$ & & $\ldots$ & $18.49 \pm 0.04$ & $18.91 \pm 0.09$ & $\ldots$ & $17.063 \pm 0.052$ & $15.134 \pm 0.022$ & $1.929 \pm 0.057$ \\
\hline & 4 & $\ldots$ & $>18.93$ & $>18.17$ & $>16.75$ & $\ldots$ & $\ldots$ & $\ldots$ & $\ldots$ & $\ldots$ & $\ldots$ \\
\hline WISE J1728+5716 & 6 & $18.59 \pm 0.05$ & $17.68 \pm 0.05$ & $17.88 \pm 0.07$ & $\ldots$ & $\ldots$ & $\ldots$ & $\ldots$ & $16.469 \pm 0.037$ & $14.986 \pm 0.021$ & $1.484 \pm 0.042$ \\
\hline WISE J1738+2732 & 7 & $\ldots$ & $\ldots$ & $\ldots$ & $\ldots$ & $19.47 \pm 0.08$ & $20.66 \pm 0.38$ & $\ldots$ & $17.097 \pm 0.053$ & $14.476 \pm 0.019$ & $2.621 \pm 0.057$ \\
\hline \multirow[t]{3}{*}{ WISE J1741+2553 } & 1 & $\ldots$ & $16.451 \pm 0.099$ & $16.356 \pm 0.216$ & $>16.785$ & $\ldots$ & $\ldots$ & $\ldots$ & $14.428 \pm 0.020$ & $12.388 \pm 0.017$ & $2.040 \pm 0.026$ \\
\hline & 4 & $\ldots$ & $16.48 \pm 0.02$ & $16.24 \pm 0.04$ & $16.89 \pm 0.20$ & $\ldots$ & $\ldots$ & $\ldots$ & $\ldots$ & $\ldots$ & $\ldots$ \\
\hline & 6 & $17.23 \pm 0.02$ & $16.53 \pm 0.02$ & $16.63 \pm 0.03$ & $\ldots$ & $\ldots$ & $\ldots$ & $\ldots$ & $\ldots$ & $\ldots$ & $\ldots$ \\
\hline \multirow[t]{2}{*}{ WISE J1804+3117 } & 7 & $\ldots$ & $\ldots$ & $\ldots$ & $\ldots$ & $18.70 \pm 0.05$ & $19.21 \pm 0.11$ & $\ldots$ & $16.616 \pm 0.039$ & $14.602 \pm 0.020$ & $2.014 \pm 0.044$ \\
\hline & 4 & $\ldots$ & $>18.88$ & $>18.24$ & $>16.86$ & $\ldots$ & $\ldots$ & $\ldots$ & $\ldots$ & $\ldots$ & $\ldots$ \\
\hline \multirow[t]{2}{*}{ WISE J1812+2721 } & 7 & $\ldots$ & $\ldots$ & $\ldots$ & $\ldots$ & $18.19 \pm 0.06$ & $18.83 \pm 0.16$ & $\ldots$ & $\ldots$ & $\ldots$ & $\ldots$ \\
\hline & 4 & $\ldots$ & $>18.58$ & $>17.77$ & $>17.03$ & $\ldots$ & $\ldots$ & $\ldots$ & $\ldots$ & $\ldots$ & $\ldots$ \\
\hline WISE J1828+2650 & 11 & $\ldots$ & $\ldots$ & $\ldots$ & $\ldots$ & $23.57 \pm 0.35$ & $22.85 \pm 0.24$ & $\ldots$ & $16.917 \pm 0.020$ & $14.322 \pm 0.020$ & $2.595 \pm 0.028$ \\
\hline WISE J1830+4542 & 2 & $\ldots$ & $>18.752$ & $16.082 \pm 0.179$ & $15.369 \pm 0.183$ & $\ldots$ & $\ldots$ & $\ldots$ & $\ldots$ & .. & $\ldots$ \\
\hline \multirow[t]{3}{*}{ WISE J1841+7000 } & 2 & $\ldots$ & $17.211 \pm 0.250$ & $16.544 \pm 0.241$ & $>15.626$ & $\ldots$ & $\ldots$ & $\ldots$ & $15.630 \pm 0.026$ & $14.331 \pm 0.019$ & $1.299 \pm 0.032$ \\
\hline & 7 & $\ldots$ & $\ldots$ & $\ldots$ & $\ldots$ & $16.64 \pm 0.03$ & $16.99 \pm 0.04$ & $\ldots$ & $\ldots$ & $\ldots$ & $\ldots$ \\
\hline & 4 & $\ldots$ & $16.800 \pm 0.035$ & $16.912 \pm 0.082$ & $\ldots$ & $\ldots$ & $\ldots$ & $\ldots$ & $\ldots$ & $\ldots$ & $\ldots$ \\
\hline \multirow[t]{2}{*}{ WISE J1852+3537 } & 1 & $\ldots$ & $16.501 \pm 0.116$ & $>17.407$ & $>17.076$ & $\ldots$ & $\ldots$ & $\ldots$ & $15.587 \pm 0.026$ & $14.188 \pm 0.019$ & $1.399 \pm 0.032$ \\
\hline & 4 & $\cdots$ & $>16.70$ & $>15.88$ & $\cdots$ & $\cdots$ & $\cdots$ & $\cdots$ & $\cdots$ & $\cdots$ & $\cdots$ \\
\hline
\end{tabular}


Table 3

(Continued)

\begin{tabular}{|c|c|c|c|c|c|c|c|c|c|c|c|}
\hline \multirow{2}{*}{$\begin{array}{l}\text { Object } \\
\text { Name }\end{array}$} & \multirow{2}{*}{$\begin{array}{l}\text { Obs. } \\
\text { Ref. }\end{array}$} & \multirow{2}{*}{$\begin{array}{c}Y \\
(\mathrm{mag})\end{array}$} & \multicolumn{3}{|c|}{ Two Micron All-Sky Survey Filter System } & \multicolumn{3}{|c|}{ Mauna Kea Observatories Filter System } & \multicolumn{3}{|c|}{ Spitzer/IRAC Observations } \\
\hline & & & $\begin{array}{c}J \\
(\mathrm{mag})\end{array}$ & $\begin{array}{c}H \\
(\mathrm{mag})\end{array}$ & $\begin{array}{c}K_{s} \\
(\mathrm{mag})\end{array}$ & $\begin{array}{c}J \\
(\mathrm{mag})\end{array}$ & $\begin{array}{c}H \\
(\mathrm{mag})\end{array}$ & $\begin{array}{c}K_{s} \\
(\mathrm{mag})\end{array}$ & $\begin{array}{c}c h 1 \\
(\mathrm{mag})\end{array}$ & $\begin{array}{c}\text { ch2 } \\
(\mathrm{mag})\end{array}$ & $\begin{array}{c}\operatorname{ch} 1-\operatorname{ch} 2 \\
(\mathrm{mag})\end{array}$ \\
\hline (1) & (2) & (3) & (4) & $(5)$ & (6) & (7) & (8) & (9) & $(10)$ & (11) & (12) \\
\hline \multirow[t]{2}{*}{ WISE J1906+4508 } & 1 & $\ldots$ & $16.320 \pm 0.108$ & $16.129 \pm 0.214$ & $>16.107$ & $\ldots$ & $\ldots$ & $\ldots$ & $\ldots$ & $\ldots$ & $\ldots$ \\
\hline & 3 & $\ldots$ & $16.36 \pm 0.09$ & $16.32 \pm 0.09$ & $16.83 \pm 0.19$ & $\ldots$ & $\ldots$ & $\ldots$ & $\ldots$ & $\ldots$ & $\ldots$ \\
\hline WISE J1952+7240 & 4 & $\ldots$ & $15.086 \pm 0.045$ & $14.728 \pm 0.077$ & $14.650 \pm 0.078$ & $\ldots$ & $\ldots$ & $\ldots$ & $14.048 \pm 0.019$ & $13.196 \pm 0.017$ & $0.852 \pm 0.026$ \\
\hline \multirow[t]{2}{*}{ WISE J1959-3338 } & 1 & $\ldots$ & $16.866 \pm 0.145$ & $>16.077$ & $>16.227$ & $\ldots$ & $\ldots$ & $\ldots$ & $\ldots$ & $\ldots$ & $\ldots$ \\
\hline & 9 & $\ldots$ & $\ldots$ & $\ldots$ & $\ldots$ & $16.714 \pm 0.069$ & $17.178 \pm 0.054$ & $\ldots$ & $\ldots$ & $\ldots$ & $\ldots$ \\
\hline WISE J2018-7423 & $\ldots$ & $\ldots$ & $\ldots$ & $\ldots$ & $\ldots$ & $\ldots$ & $\ldots$ & $\ldots$ & $\ldots$ & $\ldots$ & $\ldots$ \\
\hline \multirow[t]{3}{*}{ WISE J2056+1459 } & 7 & $\ldots$ & $\ldots$ & $\ldots$ & $\ldots$ & $19.31 \pm 0.12$ & $>19.5$ & $\ldots$ & $16.036 \pm 0.030$ & $13.924 \pm 0.018$ & $2.112 \pm 0.036$ \\
\hline & 4 & $\ldots$ & $>17.6$ & $>17.1$ & $\ldots$ & $\ldots$ & $\ldots$ & $\ldots$ & $\ldots$ & $\ldots$ & $\ldots$ \\
\hline & 11 & $\ldots$ & $\ldots$ & $\ldots$ & $\ldots$ & $19.21 \pm 0.07$ & $19.56 \pm 0.18$ & $\ldots$ & & & \\
\hline WISE J2134-7131 & 8 & $\ldots$ & $\ldots$ & $\ldots$ & $\ldots$ & $19.8 \pm 0.1$ & $19.7 \pm 0.15$ & $\ldots$ & $16.179 \pm 0.032$ & $13.958 \pm 0.018$ & $2.221 \pm 0.037$ \\
\hline WISE J2157+2659 & 5 & $\ldots$ & $17.31 \pm 0.03$ & $17.45 \pm 0.04$ & $\ldots$ & $\ldots$ & $\ldots$ & $\ldots$ & $16.012 \pm 0.030$ & $14.438 \pm 0.019$ & $1.574 \pm 0.035$ \\
\hline WISE J2209-2734 & 1 & $\ldots$ & $16.809 \pm 0.152$ & $>16.408$ & $>16.903$ & $\ldots$ & $\ldots$ & $\ldots$ & $15.478 \pm 0.025$ & $13.900 \pm 0.018$ & $1.577 \pm 0.031$ \\
\hline \multirow[t]{4}{*}{ WISE J2213+0911 } & 2 & $\ldots$ & $17.044 \pm 0.219$ & $>16.046$ & $>16.092$ & $\ldots$ & $\ldots$ & $\ldots$ & $15.795 \pm 0.028$ & $14.565 \pm 0.020$ & $1.229 \pm 0.034$ \\
\hline & 2 & $\ldots$ & $16.834 \pm 0.187$ & $>16.071$ & $>17.175$ & $\ldots$ & $\ldots$ & $\ldots$ & $\ldots$ & $\ldots$ & $\ldots$ \\
\hline & 4 & $\ldots$ & $16.98 \pm 0.05$ & $17.09 \pm 0.11$ & $>16.2$ & $\ldots$ & $\ldots$ & $\ldots$ & $\ldots$ & $\ldots$ & $\ldots$ \\
\hline & 3 & $\ldots$ & $17.13 \pm 0.10$ & $17.32 \pm 0.14$ & $16.99 \pm 0.20$ & $\ldots$ & $\ldots$ & $\ldots$ & $\ldots$ & $\ldots$ & $\ldots$ \\
\hline WISE J2226+0440 & 4 & $\ldots$ & $17.02 \pm 0.08$ & $>17.3$ & & $\ldots$ & $\ldots$ & $\ldots$ & $16.124 \pm 0.031$ & $14.545 \pm 0.020$ & $1.579 \pm 0.037$ \\
\hline \multirow{2}{*}{ WISE J2237-0614 } & 2 & $\ldots$ & $17.182 \pm 0.191$ & $>16.009$ & $>17.054$ & $\ldots$ & $\ldots$ & $\ldots$ & $16.164 \pm 0.032$ & $14.927 \pm 0.021$ & $1.236 \pm 0.038$ \\
\hline & 4 & $\ldots$ & $17.40 \pm 0.05$ & $17.40 \pm 0.11$ & $>16.8$ & $\ldots$ & $\ldots$ & $\ldots$ & $\cdots$ & $\ldots$ & $\ldots$ \\
\hline \multirow[t]{2}{*}{ WISE J2239+1617 } & 1 & $\ldots$ & $16.079 \pm 0.077$ & $15.416 \pm 0.091$ & $>14.890$ & $\ldots$ & $\ldots$ & $\ldots$ & $14.147 \pm 0.019$ & $13.553 \pm 0.018$ & $0.594 \pm 0.026$ \\
\hline & 3 & $\ldots$ & $16.18 \pm 0.07$ & $15.50 \pm 0.07$ & $15.36 \pm 0.10$ & $\ldots$ & $\ldots$ & $\ldots$ & & & \\
\hline \multirow[t]{2}{*}{ WISE J2255-3118 } & 7 & $\ldots$ & & &. & $17.34 \pm 0.03$ & $17.70 \pm 0.11$ & $\ldots$ & $15.915 \pm 0.029$ & $14.210 \pm 0.019$ & $1.706 \pm 0.035$ \\
\hline & 4 & $\ldots$ & $17.29 \pm 0.07$ & $>17.7$ & $>16.4$ & $\ldots$ & $\ldots$ & $\ldots$ & $\ldots$ & $\ldots$ & $\ldots$ \\
\hline WISE J2313-8037 & 2 & $\ldots$ & $16.974 \pm 0.236$ & $>16.192$ & $>16.358$ & $\ldots$ & $\ldots$ & $\ldots$ & $\ldots$ & $\ldots$ & $\ldots$ \\
\hline \multirow[t]{2}{*}{ WISE J2319-1844 } & 2 & $\ldots$ & $17.433 \pm 0.229$ & $>17.225$ & $>16.009$ & $\ldots$ & $\ldots$ & $\ldots$ & $15.924 \pm 0.029$ & $13.949 \pm 0.018$ & $1.974 \pm 0.034$ \\
\hline & 7 & $\ldots$ & $\ldots$ & $\ldots$ & $\ldots$ & $17.56 \pm 0.02$ & $17.95 \pm 0.05$ & $\ldots$ & $\ldots$ & $\ldots$ & $\ldots$ \\
\hline WISE J2325-4105 & 9 & $\ldots$ & $\ldots$ & $\ldots$ & $\ldots$ & $19.745 \pm 0.050$ & $19.216 \pm 0.114$ & $\ldots$ & $16.265 \pm 0.033$ & $14.087 \pm 0.019$ & $2.178 \pm 0.038$ \\
\hline WISE J2327-2730 & 1 & $\ldots$ & $16.681 \pm 0.145$ & $15.481 \pm 0.103$ & $14.756 \pm 0.096$ & $\ldots$ & $\ldots$ & $\ldots$ & $13.584 \pm 0.018$ & $13.337 \pm 0.018$ & $0.247 \pm 0.025$ \\
\hline \multirow[t]{2}{*}{ WISE J2340-0745 } & 1 & $\ldots$ & $16.540 \pm 0.104$ & $16.212 \pm 0.193$ & $>16.271$ & $\ldots$ & $\ldots$ & $\ldots$ & $15.199 \pm 0.023$ & $13.627 \pm 0.018$ & $1.572 \pm 0.029$ \\
\hline & 4 & $\ldots$ & $16.15 \pm 0.03$ & $16.19 \pm 0.06$ & $>16.3$ & $\ldots$ & $\ldots$ & $\ldots$ & $\ldots$ & $\ldots$ & $\ldots$ \\
\hline WISE J2343-7418 & 1 & $\ldots$ & $16.132 \pm 0.091$ & $>16.142$ & $15.906 \pm 0.311$ & $\ldots$ & $\ldots$ & $\ldots$ & $\ldots$ & $\ldots$ & $\ldots$ \\
\hline WISE J2344+1034 & 7 & $\ldots$ & $\ldots$ & $\ldots$ & $\ldots$ & $18.78 \pm 0.06$ & $19.07 \pm 0.11$ & $\ldots$ & $16.734 \pm 0.042$ & $14.908 \pm 0.021$ & $1.826 \pm 0.047$ \\
\hline \multirow[t]{3}{*}{ WISE J2348-1028 } & 1 & $\ldots$ & $16.546 \pm 0.118$ & $>16.441$ & $>15.977$ & $\ldots$ & $\ldots$ & $\ldots$ & $15.869 \pm 0.028$ & $14.357 \pm 0.019$ & $1.512 \pm 0.034$ \\
\hline & 4 & $\ldots$ & $16.913 \pm 0.047$ & $17.099 \pm 0.116$ & $>16.443$ & $\ldots$ & $\ldots$ & $\ldots$ & $\ldots$ & $\ldots$ & $\ldots$ \\
\hline & 3 & $\ldots$ & $17.01 \pm 0.10$ & $16.93 \pm 0.12$ & $16.71 \pm 0.18$ & $\ldots$ & $\ldots$ & $\ldots$ & $\ldots$ & $\ldots$ & $\ldots$ \\
\hline WISE J2359-7335 & $\ldots$ & $\ldots$ & $16.160 \pm 0.105$ & $15.912 \pm 0.194$ & $>15.159$ & $\ldots$ & $\ldots$ & $\ldots$ & $14.475 \pm 0.020$ & $13.383 \pm 0.018$ & $1.092 \pm 0.026$ \\
\hline
\end{tabular}

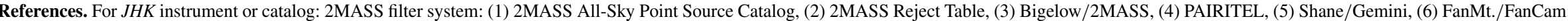
MKO filter system: (7) Palomar/WIRC, (8) Magellan/PANIC, (9) SOAR/SpartanIRC, (10) UKIDSS, (11) Keck/NIRC2. (12) CTIO-4m/NEWFIRM. 


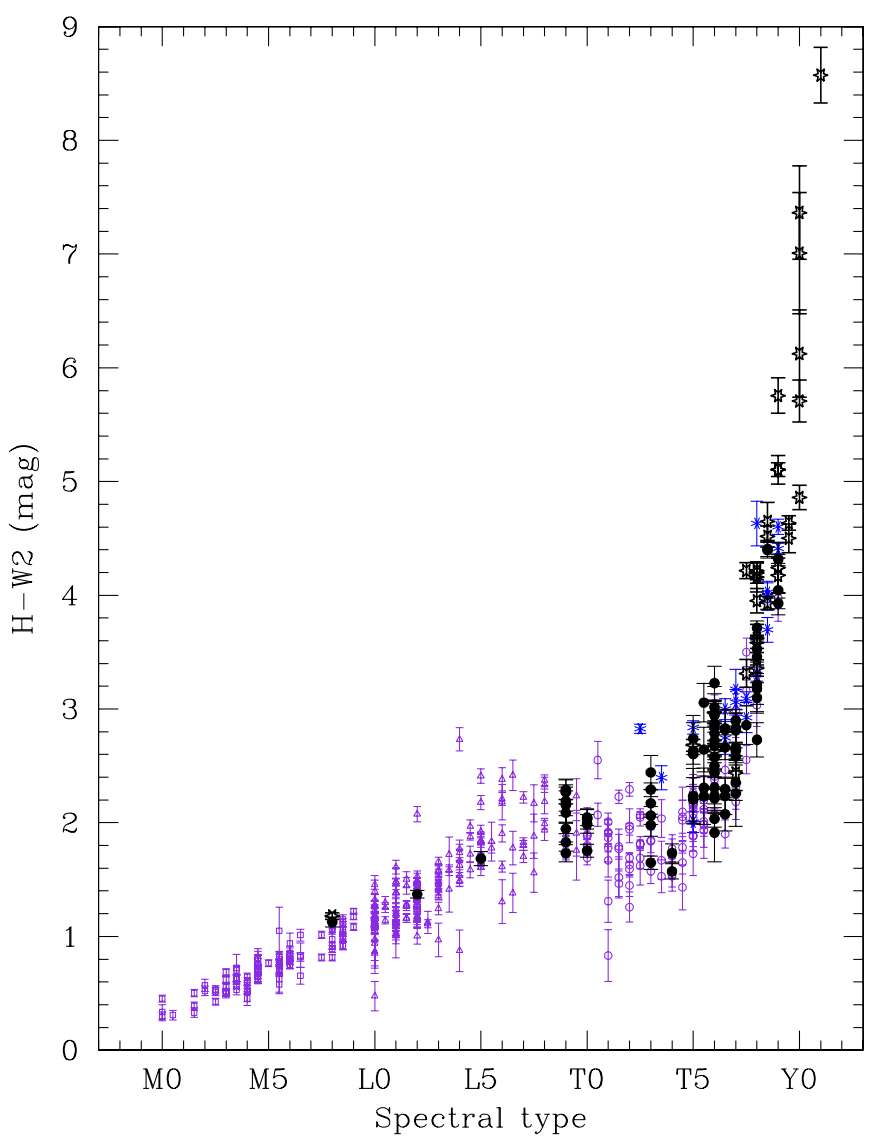

Figure 8. $H-W 2$ color vs. spectral type. Color coding is the same as in Figure 5.

(A color version of this figure is available in the online journal.)

observations were also acquired. Details on observing strategy and data reduction are described further in Mainzer et al. (2011).

\subsubsection{Magellan/PANIC}

Persson's Auxiliary Nasmyth Infrared Camera (PANIC) at the $6.5 \mathrm{~m}$ Magellan Baade Telescope has a $1024 \times 1024$ HAWAII array with a plate scale of $00^{\prime \prime} 125$ pixel $^{-1}$, resulting in a $2 \times 2$ arcmin field of view (Martini et al. 2004). Observations of three of our candidates were obtained in the Carnegie (essentially MKO) $J$ and $H$ bands. A series of dithered images of short integration times was obtained in each band, as is the standard in near-infrared imaging observations.

The data were reduced using custom Interactive Data Language (IDL) routines written by one of us (M.C.C.). Each image was first corrected for nonlinearity using the relation given in the PANIC Observer's Manual. ${ }^{31}$ Sky flat fields were constructed by first subtracting a median-averaged dark frame from each twilight flat frame. The dark-subtracted twilight flats were then scaled to a common flux level and then medianed. After each frame was sky subtracted and flat fielded, we corrected for optical distortions as described in the PANIC Observer's Manual. Frames were then aligned and combined using a median average to produce the final mosaic. 2MASS stars in the field of view were used to both astrometrically calibrate the mosaic as well as determine the zero points for the images.

\footnotetext{
31 See http://www.lco.cl/telescopes-information/magellan/instruments/ panic/panic-online-documentation/panic-manual/panic-observers-s-manual/ panic-manual
}

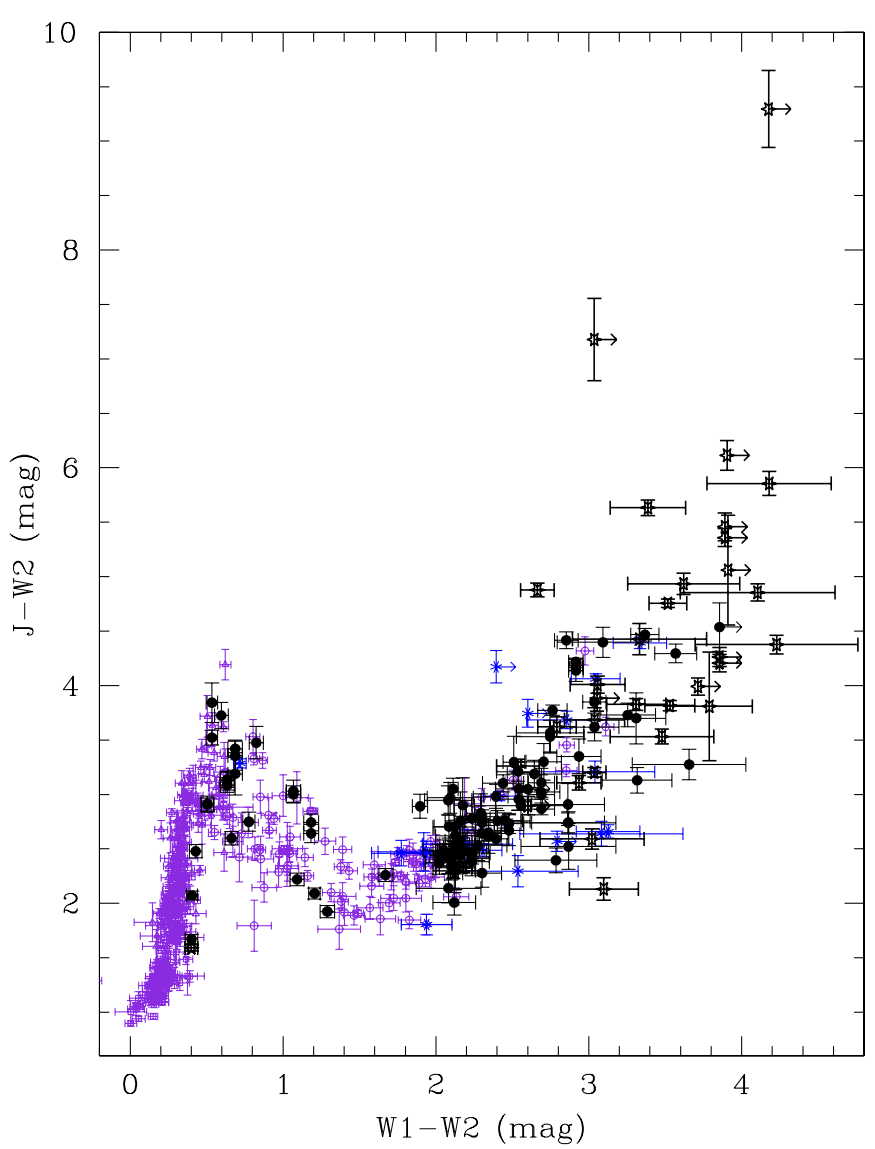

Figure 9. $J-W 2$ color plotted against $W 1-W 2$ color. Color coding is the same as in Figure 5.

(A color version of this figure is available in the online journal.)

\subsubsection{Mt. Bigelow/2MASS}

The 2MASS camera on the $1.5 \mathrm{~m}$ Kuiper Telescope on Mt. Bigelow, Arizona, has three $256 \times 256$ NICMOS3 arrays capable of observing simultaneously in 2MASS $J, H$, and $K_{s}$ filters (Milligan et al. 1996). The plate scale for all three arrays is $1^{\prime \prime} 65$ pixel $^{-1}$, resulting in a $7 \times 7$ arcmin field of view. Images for 23 of our candidates were obtained using a $3 \times 3$ box dither pattern, and to reduce the overheads associated with nodding the telescope four images were taken at each of the nine dither positions. At the conclusion of each dither sequence, the telescope was offset slightly before the start of the next sequence.

The data were reduced using custom IDL routines. Flat fields in each band were constructed using on-source frames. These images were scaled to a common flux level and combined using an average, the latter process rejecting the lowest 10 and highest 20 values at each pixel location to eliminate imprinting of real objects. Each raw frame was then flat fielded with these derived flats. Sky subtraction was then accomplished in a twostep process. First, all of the co-added frames were median averaged to produce a first-order sky frame. This frame was then subtracted from each of the co-added frames so that stars could be easily identified. A sky frame was then constructed for each co-added image using the five previous frames in the sequence and the next five frames in the sequence. Stars identified in the previous step were masked out before the 10 frames were median averaged. The sky frame was then scaled to the same flux level as the image and subtracted. After all of the co-added frames 


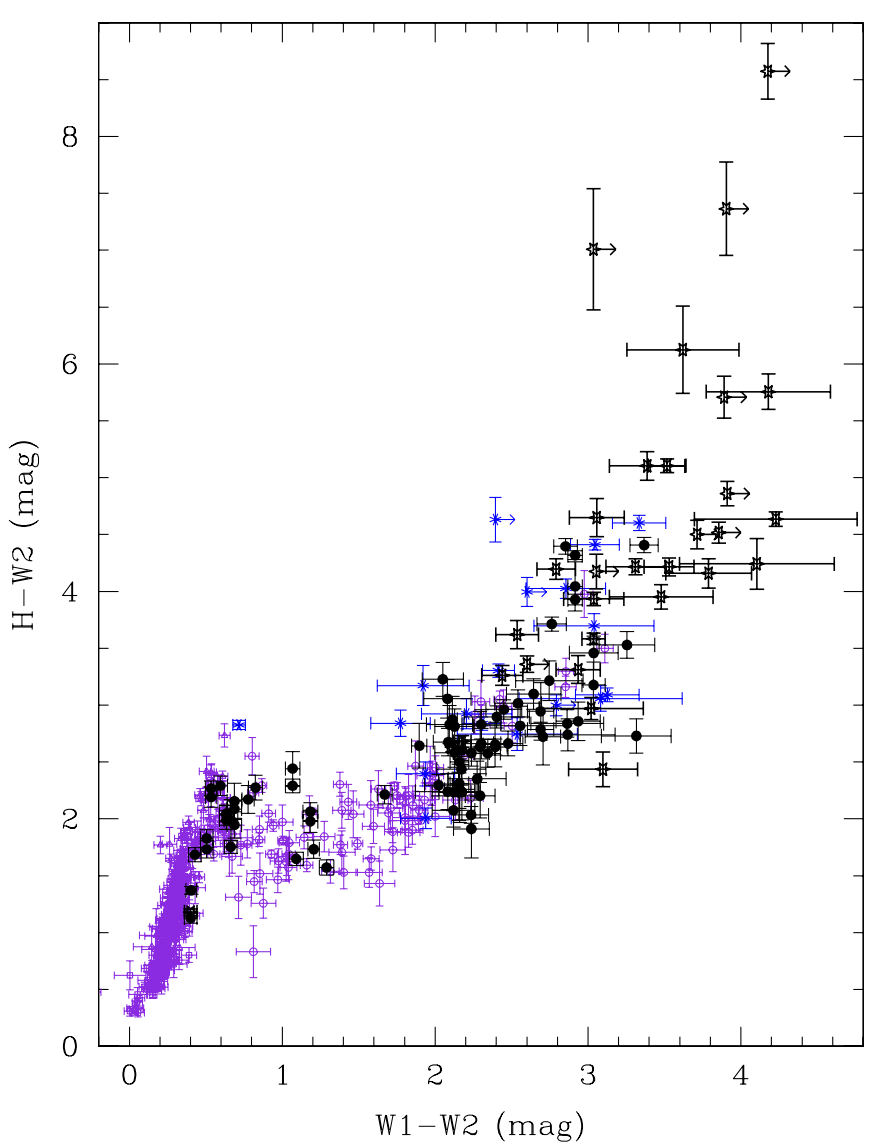

Figure 10. $H-W 2$ color plotted against $W 1-W 2$ color. Color coding is the same as in Figure 5.

(A color version of this figure is available in the online journal.)

had been sky subtracted, the frames were co-registered on the sky and averaged. 2MASS stars in the field of view were used to both astrometrically calibrate the mosaic as well as determine the zero points for the images.

\subsubsection{PAIRITEL}

The Peters Automated Infrared Imaging Telescope (PAIRITEL; Bloom et al. 2006) on Mt. Hopkins, Arizona, is a $1.3 \mathrm{~m}$ telescope equipped with three $256 \times 256$ NICMOS3 arrays with $2^{\prime \prime}$ pixels that cover an $8.5 \times 8.5$ arcmin field of view simultaneously in 2MASS $J, H$, and $K_{s}$ filters (Milligan et al. 1996). This camera is, in fact, one of the original 2MASS cameras (Skrutskie et al. 2006) and the telescope is the original 2MASS northern facility, now roboticized for transient followup and other projects. Thirty-three of our candidates were observed here.

Upon acquiring a target field, the system acquires a series of $7.8 \mathrm{~s}$ exposures until the desired integration time (1200 s) is met. After every third exposure the telescope shifts to a position offset by about one-tenth of the field of view in order to account for bad pixels on the arrays. Within hours of a night's observations an automated data pipeline delivers Flexible Image Transport System (FITS; Hanisch et al. 2001) mosaic images of the combined stack of observations for each object in each of the three wavebands. The pipeline subtracts a background from each raw image based on the median of several images adjacent in time. The resulting individual frames are calibrated with a flat field constructed from sky observations spanning many nights.
Astrometric and photometric calibrations were accomplished using observations of 2MASS-detected stars in the field of view.

\subsubsection{Palomar/WIRC}

The Wide-field Infrared Camera (WIRC) at the $5 \mathrm{~m}$ Hale Telescope at Palomar Observatory has a pixel scale of 0 '.2487 pixel $^{-1}$ and uses a $2048 \times 2048$ pixel HAWAII-2 array to image an 8.7 arcmin square field of view (Wilson et al. 2003a). Observations of 18 of our candidates were obtained in the MKO $J$ and $H$ bands.

Images were reduced using a suite of IRAF $^{32}$ scripts and FORTRAN programs kindly provided by T. Jarrett. The images were first linearized and dark subtracted. Sky-background and flat-field images were created from the list of input images, and then these were subtracted from and divided into, respectively, each input image. At this stage, WIRC images still contained a significant bias not removed by the flat field. Comparison of 2MASS and WIRC photometric differences across the array showed that this flux bias had a level of $\approx 10 \%$ and the pattern was roughly the same for all filters. Using these 2MASS-WIRC differences for many fields, we created a flux bias correction image that was then applied to each of the "reduced" images. Finally, we astrometrically calibrated the images using 2MASS stars in the field. The images were then mosaicked together. This final mosaic was photometrically calibrated using 2MASS stars and a custom IDL script. Magnitudes were calculated using the zero points computed using 2MASS stars.

\subsubsection{Shane/Gemini}

The Gemini Infrared Imaging Camera at the $3 \mathrm{~m}$ Shane Telescope at Lick Observatory uses two $256 \times 256$ pixel arrays (pixel scale of 0.70 arcsec pixel $^{-1}$ ) for simultaneous observations of a $3 \times 3$ arcmin field of view (McLean et al. 1994) over each array. The short-wavelength channel uses a NICMOS3 HgCdTe array, and the long-wavelength channel uses an InSb array. Observations were obtained for three of our candidates and used 2MASS $J, H$, and $K_{s}$ filters. Observations were acquired in pairs $\left(J+K_{s}\right.$ or $\left.H+K_{s}\right)$ so that twice as much integration time could be obtained on the InSb array at $K_{s}$ as in either the $J$ or $H$ filter on the $\mathrm{HgCdTe}$ array. Observations were obtained in dithered sequences. Data reduction was handled similarly to that described for the Mt. Bigelow/2MASS data.

\subsubsection{SOAR/SpartanIRC}

The Spartan Infrared Camera (SpartanIRC) at the $4.1 \mathrm{~m}$ Southern Astrophysics Research (SOAR) Telescope on Cerro Pachón, Chile, uses a mosaic of two $2048 \times 2048$ pixel HAWAII-2 arrays to cover either a 3 arcmin square $(0.04$ arcsec pixel $\left.^{-1}\right)$ or 5 arcmin square $\left(0.07\right.$ arcsec pixel $\left.{ }^{-1}\right)$ field of view (Loh et al. 2004). For seven of our candidates, we used the $5 \times 5$ arcmin mode and acquired observations in the MKO $J$ and $H$ (and for one object, $K$ ) filters. Observing strategy and data reductions followed the same prescription discussed in Burgasser et al. (2011a).

\subsubsection{Keck/NIRC2}

The second-generation Near-infrared Camera (NIRC2) at the $10 \mathrm{~m}$ W. M. Keck Observatory atop Mauna Kea, Hawai'i,

\footnotetext{
32 The Image Reduction and Analysis Facility (Tody 1986) is distributed by the National Optical Astronomy Observatory, which is operated by the Association of Universities for Research in Astronomy, Inc., under cooperative agreement with the National Science Foundation.
} 
employs a $1024 \times 1024$ Aladdin-3 array. Used with the Keck II laser guide star adaptive optics system (Wizinowich et al. 2006; van Dam et al. 2006), it can provide deep, high-resolution, imaging for our faintest targets. In the wide-field mode, the camera has a plate scale of $0.039686 \mathrm{arcsec}_{\mathrm{pixel}}{ }^{-1}$ resulting in a field of view of $40 \times 40 \mathrm{arcsec}$. WISEPA J182831.08+265037.8 and WISEPC J205628.90+145953.3 are the only targets in this paper whose photometry comes solely from the NIRC2 data because they were undetected in $J$ and/or $H$ band at other facilities. The camera employs MKO $J$ and $H$ filters.

For WISEPA J182831.08+265037.8 we used the $R=16$ star USNO-B 1168-0346313 (Monet et al. 2003), located 41" from the target, for the tip-tilt reference star. The tip-tilt reference star for WISEPC J205628.90+145953.3 was USNO-B 1050$0583683\left(R=13\right.$, separation $\left.=13^{\prime \prime}\right)$. A three-position dither pattern was used to avoid the noisy lower-left quadrant. Each position of the dither pattern consisted of a 120 s exposure; the pattern was repeated two or three times with a different offset for each repeat in order to build up deeper exposures.

The images were reduced using a custom set of IDL scripts written by one of us (C.R.G.). The raw images were first sky-subtracted using a sky frame constructed from all of the images. Next, a dome flat was used to correct for pixelto-pixel sensitivity variations. In order to shift the reduced images to a common astrometric grid for the creation of the mosaic, we used the header keywords AOTSX and AOTSY, which record the position of the AO tip-tilt sensor stage. As the telescope is dithered, this sensor must move so that the tip-tilt star stays properly centered. Although this method of computing image offsets is more precise than using the right ascension and declination offsets in the header, it can be prone to small positional errors. To account for this, we computed the minimum of the residuals of the shifted image relative to the reference image (the first image of the stack) over a $5 \times$ 5 pixel grid centered on the AOTSX/AOTSY-computed offset. This correction was generally $<2$ pixels. The aligned images were then medianed to form the final mosaic.

There are typically very few, if any, 2MASS stars in the NIRC2 field of view that can be used to determine the photometric zero point. We therefore bootstrapped a photometric zero point using faint stars in the NIRC2 images that are also present in the much wider field-of-view Palomar/WIRC images that have been calibrated using 2MASS stars. For this ensemble we computed the average difference between the calibrated WIRC magnitudes and the NIRC2 instrumental magnitudes. We used the standard deviation of the differences as the error in the photometric calibration and note that it is the dominant source of uncertainty.

\subsubsection{CTIO-4m/NEWFIRM}

The NOAO Extremely Wide Field Infrared Imager (NEWFIRM) at the $4 \mathrm{~m}$ Victor M. Blanco Telescope on Cerro Tololo, Chile, consists of four $2048 \times 2048 \mathrm{InSb}$ arrays arranged in a $2 \times 2$ grid. With a pixel scale of $0.40 \operatorname{arcsec}_{\text {pixel }}{ }^{-1}$, this grid covers a total field of view of $27.6 \times 27.6$ arcmin (Swaters et al. 2009). The only source in this paper with NEWFIRM photometry is WISEPA J1541521.66-225025.2, so the discussion that follows is specific to this set of observations.

At the $J$ band, 10 sets of images were obtained with integration times of $30 \mathrm{~s}$ and 2 co-adds; at the $H$ band, 10 sets with exposure times of $5 \mathrm{~s}$ and 12 co-adds were obtained. Thus, the total integration time was $600 \mathrm{~s}$ in each filter, and these are on the MKO system. Because of the large field of view and the $35^{\prime \prime}$ gap between the arrays, we positioned the target near the center of the northeast array, designated SN013 in the Proposal Preparation Guide. $^{33}$

Photometry was performed on the mosaics produced by the NEWFIRM pipeline (Swaters et al. 2009). Although the pipeline computed a magnitude zero-point based on the photometry of 2MASS stars in the mosaic, the default aperture used was so large ( $\approx 6$ pixel radius) that WISEPA J1541521.66-225025.2 overlapped with a close neighbor. Furthermore, WISEPA J1541521.66-225025.2 was barely visible on the mosaics, meaning that a smaller aperture was warranted to increase the $S$ / $\mathrm{N}$ of our measurement. We therefore re-calibrated the mosaics using an aperture of 2 pixel radius.

\subsubsection{MASS and UKIDSS}

Some of the WISE brown dwarf candidates were detected by 2MASS or by the United Kingdom Infrared Deep Sky Survey (UKIDSS; Lawrence et al. 2007). For 2MASS, we have searched the All-Sky Point Source Catalog and the Reject Table ${ }^{34}$ and found photometry for 45 of our sources, the other 51 being too faint for 2MASS detection. For UKIDSS, we searched Data Release 6 (or 5 for those mini-surveys not released in 6) and found five of our objects-three in the Large Area Survey and two in the Galactic Clusters Survey.

\subsection{Follow-up Using Spitzer/IRAC}

Some of the coldest brown dwarf candidates detected by WISE have $W 1-W 2$ color limits because these sources are too faint to be detected in the $W 1$ band. Acquiring deeper images at similar bandpasses would therefore be extremely beneficial in further deciding which of the candidates are the most interesting for spectroscopic follow-up. Fortunately, the two shortest wavelength bandpasses of the IRAC (Fazio et al. 2004) on board the Spitzer Space Telescope (Werner et al. 2004) are operating during the Warm Spitzer mission and can be used to provide this missing info. Although these bandpasses-at 3.6 and $4.5 \mu \mathrm{m}$ (also known as $\operatorname{ch} 1$ and $\operatorname{ch} 2$, respectively) - are similar to the two shortest wavelength bands of WISE (see Figure 2 of Mainzer et al. 2011), they were not designed specifically for brown dwarf detection and therefore yield less extreme colors (ch $1-\operatorname{ch} 2$ versus $W 1-W 2$ ) for objects of the same spectral type. Nonetheless, IRAC photometry in the Warm Spitzer era provides a powerful verification tool for WISE brown dwarf candidates.

Figure 11 shows the trend of $\operatorname{ch} 1-\operatorname{ch} 2$ color versus spectral type for early-M through late-T dwarfs taken from Patten et al. (2006), Leggett et al. (2009), and Leggett et al. (2010). As noted by these authors, there is a clear trend of increasing $\operatorname{ch} 1-\operatorname{ch} 2$ color with later spectral type, as expected. Figure 12 maps the ch $1-$ ch 2 color onto $W 1-W 2$ color for these same sources. Two additional plots, Figures 13 and 14, show composite colors made from ground-based near-infrared and Spitzer follow-up and demonstrate that both $J-\operatorname{ch} 2$ and $H-\operatorname{ch} 2$ colors can serve as proxies for spectral type for objects of type mid-T and later. Plots of $H-c h 2$ color versus type have been presented in earlier papers (the upper left-hand panel of Figure 6 of Leggett et al. 2010; Figure 5 of Eisenhardt et al. 2010). Even earlier, Warren et al. (2007) noted the tight correlation between $H-c h 2$

\footnotetext{
33 http://www.noao.edu/ets/newfirm/documents/Quick_Guide_for_ Proposal_Preparation.pdf

34 See http://www.ipac.caltech.edu/2mass/releases/allsky/doc/explsup.html for details.
} 


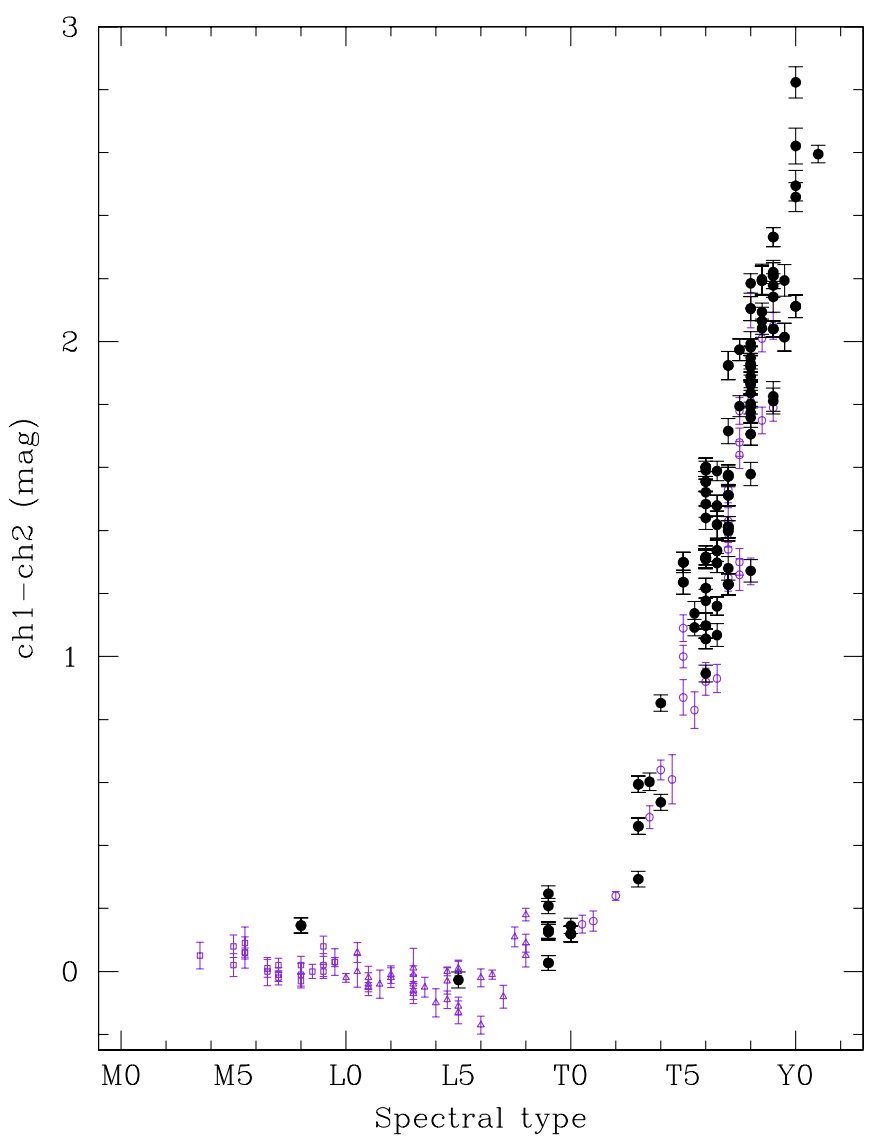

Figure 11. Spitzer/IRAC $\operatorname{ch} 1-\operatorname{ch} 2$ color as a function of spectral type. The color scheme is identical to that of Figure 1.

(A color version of this figure is available in the online journal.)

color and effective temperature for later $\mathrm{T}$ dwarfs and suggested that the color is relatively insensitive to gravity and metallicity, which makes it a reliable indicator of $T_{\text {eff }}$ or type (see also Burningham et al. 2008).

We have therefore obtained IRAC $\operatorname{ch} 1$ and $\operatorname{ch} 2$ photometry of many of our WISE brown dwarf candidates (Program ID $=70062$; PI: J. D. Kirkpatrick). Data were collected in both channels using a five-position, medium-scale "cycling" dither script. Single images were $30 \mathrm{~s}$ per position, for a total on-source exposure time of $150 \mathrm{~s}$ per channel.

Although the Spitzer Science Center (SSC) suggests performing photometry on custom-built mosaics from the basic calibrated data $(\mathrm{BCD})$ rather than the post-BCD mosaics, we have found that the photometric differences between the two mosaics are negligible for our data sets. Therefore, photometry of our sources was measured on the post-BCD mosaics as produced by the SSC IRAC Pipeline, software version S18.18. The postBCD mosaics have a pixel scale of $0 . ' 6$ pixel $^{-1}$, which is half of the native pixel scale. We used a 4 pixel aperture, a sky annulus of 24-40 pixels, and applied the aperture corrections listed in Table 4.7 of the IRAC Instrument Handbook ${ }^{35}$ to account for our non-standard aperture size. Resulting IRAC photometry for those confirmed candidates observed so far is listed in Table 3.

\section{FOLLOW-UP SPECTROSCOPIC OBSERVATIONS}

For candidates whose follow-up imaging strengthened their credibility as a bona fide brown dwarf, we obtained spectro-

\footnotetext{
35 http://ssc.spitzer.caltech.edu/irac/iracinstrumenthandbook
}

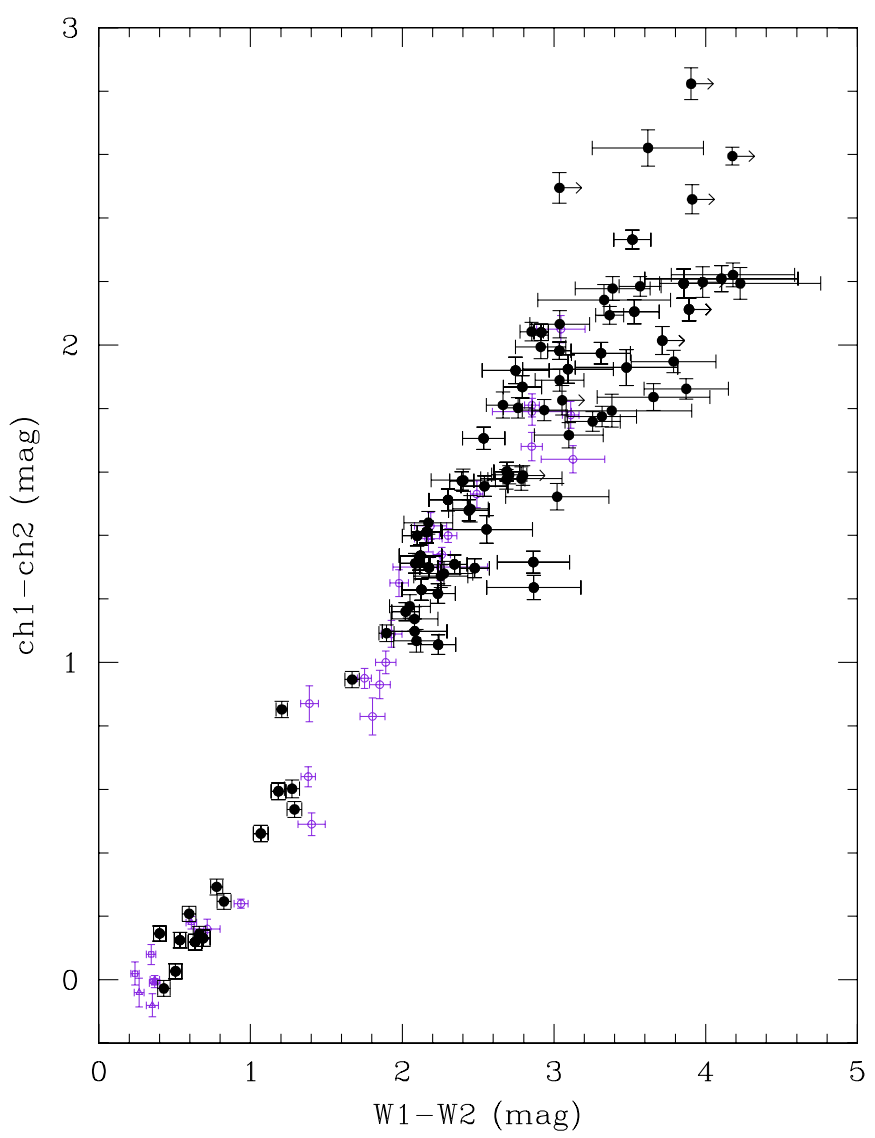

Figure 12. Spitzer/IRAC ch $1-\operatorname{ch} 2$ color as a function of $W 1-W 2$ color. The color scheme is identical to that of Figure 1.

(A color version of this figure is available in the online journal.)

scopic confirmation at near-infrared wavelengths. The facilities and instruments we have used, along with the data reductions employed, are discussed below.

\subsection{Keck/LRIS}

The Low Resolution Imaging Spectrometer (LRIS; Oke et al. 1995) at the $10 \mathrm{~m} \mathrm{~W}$. M. Keck Observatory atop Mauna Kea, Hawai'i, uses two channels to simultaneously observe blue and red optical wavelengths. Our observational setup employed only the two $2 \mathrm{k} \times 4 \mathrm{k}$ CCDs in the red channel. When used with the 400 lines $\mathrm{mm}^{-1}$ grating blazed at $8500 \AA$ and a $1^{\prime \prime}$ slit, the red channel produces $10 \AA$ resolution spectra covering the range from 6300 to $10100 \AA$. The OG570 orderblocking filter was used to eliminate second-order light. Flatfield exposures of the interior of the telescope dome were used to normalize the response of the detector, and $\mathrm{HgNeAr}$ arc lamps were taken after each program object to obtain the wavelength calibration. Because our targets were late-T dwarfs, for which the $9300 \AA$ band of $\mathrm{H}_{2} \mathrm{O}$ is the most important spectral diagnostic, observations of G0 dwarf stars near in airmass and near in time were needed to correct for telluric $\mathrm{H}_{2} \mathrm{O}$ at these same wavelengths. Observations were typically acquired with the slit oriented to the parallactic angle to minimize wavelengthdependent slit losses. Once per night, a standard star from the list of Hamuy et al. (1994) was observed to provide flux calibration. The data were reduced and calibrated using standard IRAF routines as described in Kirkpatrick et al. (1999) and Kirkpatrick et al. (2006). 


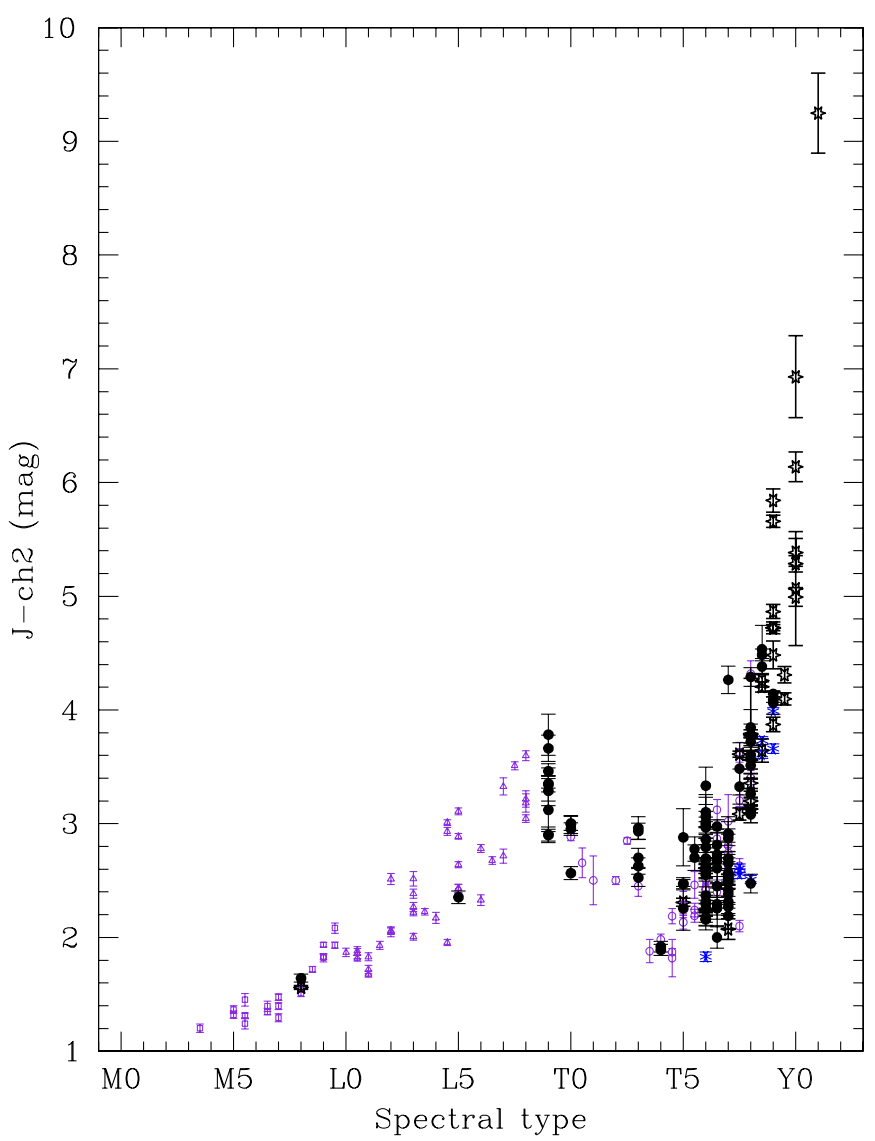

Figure 13. $J-\operatorname{ch} 2$ color as a function of spectral type. Color coding is the same as in Figure 5.

(A color version of this figure is available in the online journal.)

\subsection{IRTF/SpeX}

SpeX, a medium-resolution spectrograph and imager at NASA's 3 m Infrared Telescope Facility (IRTF) on Mauna Kea, Hawai'i, uses a $1024 \times 1024$ InSb array for its spectroscopic observations (Rayner et al. 2003). We used the prism mode with a 0 .'5 wide slit to achieve a resolving power of $R \equiv \lambda / \Delta \lambda \approx 150$ over the range $0.8-2.5 \mu \mathrm{m}$. A series of $120 \mathrm{~s}$ or $180 \mathrm{~s}$ exposures were typically obtained at two different nod positions along the $15^{\prime \prime}$ long slit. A0 dwarf stars at similar airmass to the target were observed near in time for telluric correction and flux calibration. Observations were typically obtained with the slit oriented to the parallactic angle to minimize slit loses and spectral slope variations due to differential atmospheric refraction. Finally, a set of exposures of internal flat field and argon arc lamps were obtained for flat fielding and wavelength calibration.

The data were reduced using Spextool (Cushing et al. 2004) the IDL-based data reduction package for SpeX. The raw images were first corrected for nonlinearity, pair subtracted, and then flat fielded. For some of the fainter sources, multiple pairsubtracted images were averaged in order to facilitate tracing. The spectra were then optimally extracted (e.g., Horne 1986) and wavelength calibrated using the argon lamp exposures. Multiple spectra were then averaged and the resulting spectrum was corrected for telluric absorption and flux calibrated using observations of an $\mathrm{A} 0 \mathrm{~V}$ star using the technique described in Vacca et al. (2003).

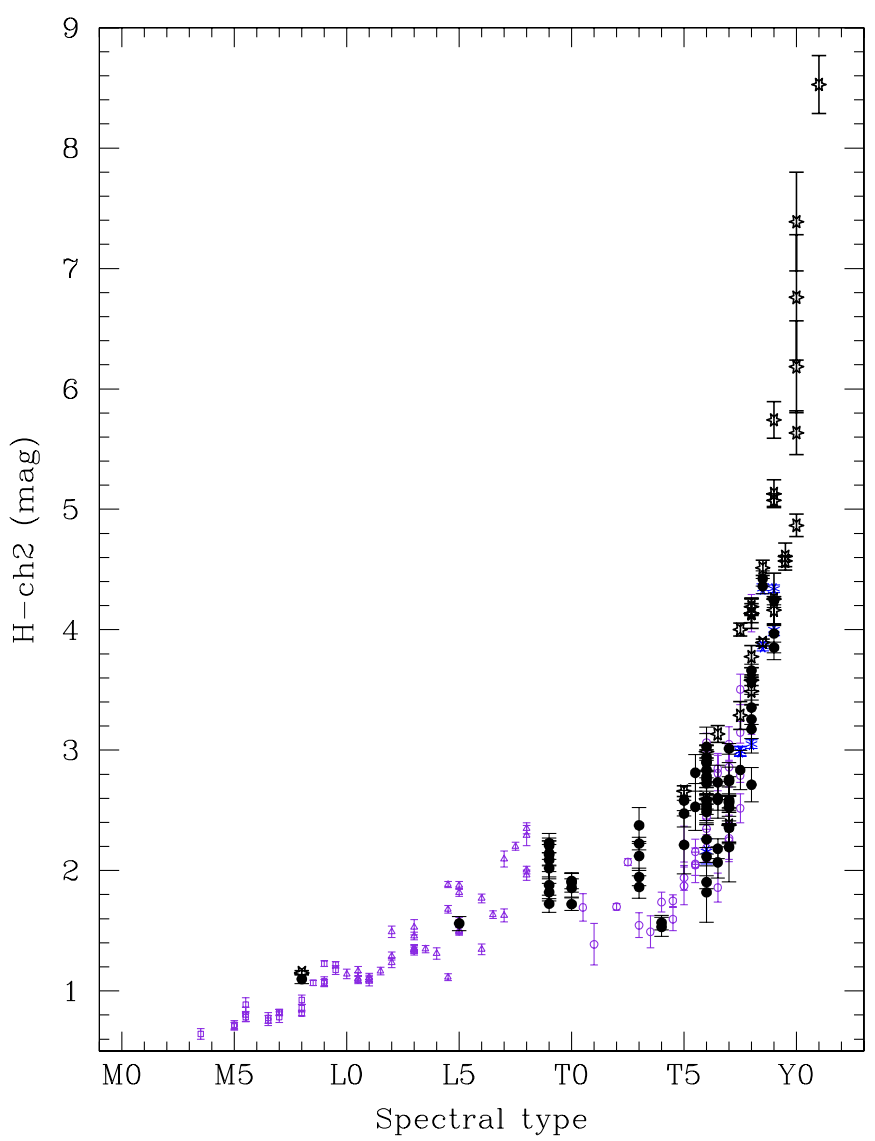

Figure 14. $H-\operatorname{ch} 2$ color as a function of spectral type. Color coding is the same as in Figure 5.

(A color version of this figure is available in the online journal.)

\subsection{Keck/NIRSPEC}

The Near-Infrared Spectrometer (NIRSPEC; McLean et al. $1998,2000)$ at the $10 \mathrm{~m} \mathrm{W.} \mathrm{M.} \mathrm{Keck} \mathrm{Observatory} \mathrm{on} \mathrm{Mauna}$ Kea, Hawai'i, uses a $1024 \times 1024 \mathrm{InSb}$ array for spectroscopy. In a low-resolution mode, use of the $42^{\prime \prime} \times 0.38$ slit results in a resolving power of $R \equiv \lambda / \Delta \lambda \approx 2500$. Our brown dwarf candidates were observed in either or both of the N3 and N5 configurations (see McLean et al. 2003) that cover the portion of the $J$-band window from 1.15 to $1.35 \mu \mathrm{m}$ and the portion of the $H$-band window from 1.5 to $1.8 \mu \mathrm{m}$.

Data were typically obtained in four or more sets of dithered pairs, with a $300 \mathrm{~s}$ exposure obtained at each dither position. To measure telluric absorption and to calibrate the flux levels, A0 dwarf stars were observed near in time and airmass to the target. Other calibrations consisted of neon and argon arc lamp spectra, dark frames, and spectra of a flat-field lamp. We employed standard reductions using the REDSPEC package, as described in McLean et al. (2003).

\subsection{Magellan/FIRE}

The Folded-port Infrared Echellette (FIRE; Simcoe et al. 2008, 2010) at the $6.5 \mathrm{~m}$ Magellan Baade Telescope uses a $2048 \times 2048$ HAWAII-2RG array. In its high-throughput prism mode, it covers the wavelength range from 0.8 to $2.5 \mu \mathrm{m}$ at a resolution ranging from $R=500$ at the $J$ band to $R=300$ at the $K$ band for a slit width of 0!6. Typically, each observation used the $50^{\prime \prime}$ long slit aligned to the parallactic angle, and consisted of a series of nod pairs taken with exposure times not exceeding 
120 s per position. The spectrograph detector was read out using the four-amplifier mode at "high gain" (1.2 counts per $\left.e^{-}\right)$with the Fowler-8 sampling mode. We also obtained exposures of a variable voltage quartz lamp (set at $1.2 \mathrm{~V}$ and $2.2 \mathrm{~V}$ ) for flat-fielding purposes and neon/argon arc lamps were used for wavelength calibration. A0 dwarf stars were used for telluric and flux calibration.

Data were reduced using a modified version of Spextool. In contrast to SpeX, the spatial axis of the FIRE slit is not aligned with the columns of the detector so that the wavelength solution is not only a function of the column number but also of the row number. We therefore derived a two-dimensional (2D) wavelength solution in two steps. First, a one-dimensional (1D) wavelength solution applicable to the center of the slit was determined using the night-sky $\mathrm{OH}$ emission lines (Cushing et al. 2004). Second, the $\mathrm{OH}$ emission lines were used to map the optical distortions in the spatial and dispersion axes within each order. The 1D wavelength solution and distortion maps were then combined to assign a wavelength and spatial position to each pixel in each order.

With the wavelength calibration completed, the remainder of the reduction steps could proceed. Pairs of images taken at different positions along the slit were subtracted in order to remove the bias and dark current as well as to perform a first-order sky subtraction. The resulting pair-subtracted image was then flat fielded using a normalized flat constructed from dome flats taken at the start of each night. The spectral extraction followed the technique described in Smith et al. (2007). A set of "pseudorectangles" was defined spanning each order to map out positions of constant wavelength on the detector. These rectangles are themselves composed of pseudopixels with a width and height of $\sim 1$ detector pixel. The pseudorectangles were then extracted using a polygon clipping algorithm (Sutherland \& Hodgman 1974), producing 1D profiles of the slit at each wavelength. Spectral extraction including residual background subtraction could then proceed in the standard way. The raw spectra were then combined and corrected for telluric absorption and flux calibrated using the observations of an $\mathrm{A} 0 \mathrm{~V}$ star and the technique described in Vacca et al. (2003).

\subsection{Palomar/TSpec}

The Triple Spectrograph (TSpec) at the $5 \mathrm{~m}$ Hale Telescope at Palomar Observatory uses a $1024 \times 2048$ HAWAII-2 array to cover simultaneously the range from 1.0 to $2.45 \mu \mathrm{m}$ (Herter et al. 2008). With a $1 \times 30$ arcsec slit, it achieves a resolution of $\sim 2700$. Observations were acquired in an ABBA nod sequence with an exposure time per nod position not exceeding $300 \mathrm{~s}$ to mitigate problems with changing $\mathrm{OH}$ background levels. Observations of A0 dwarf stars were taken near in time and near in airmass to the target objects and were used for telluric correction and flux calibration. Dome flats were taken to calibrate the pixel-to-pixel response. Data reduction is identical to that discussed above for FIRE because Spextool required the same changes for TSpec reductions as it did for FIRE reductions.

\subsection{SOAR/OSIRIS}

The Ohio State Infrared Imager/Spectrometer (OSIRIS) mounted at the $4.1 \mathrm{~m}$ Southern Astrophysical Research Telescope (SOAR) located at Cerro Pachón, Chile, uses a $1024 \times$ $1024 \mathrm{HgCdTe}$ array. The $1^{\prime \prime} .0$ wide slit yields a resolving power of $R \approx 1400$ across the $1.18-2.35 \mu \mathrm{m}$ wavelength range in three spectral orders. Short exposures (180 s) were taken at six or seven positions nodded along the $24^{\prime \prime}$ long slit. A0 dwarf stars were observed for telluric correction and flux calibration. Wavelength calibration was based on the $\mathrm{OH}$ airglow lines. The data were reduced using a modified version of the Spextool data reduction package. (See Section 4.2 for a description of Spextool.) The three spectral orders were then merged into a single spectrum covering the entire wavelength range.

\section{7. $H S T / W F C 3$}

The Wide Field Camera 3 (WFC3) on board the Hubble Space Telescope $(H S T)$ employs a $1024 \times 1024 \mathrm{HgCdTe}$ detector with a plate scale of 0.13 pixel $^{-1}$ to image a field of view of $123 \times 126$ arcsec. We used the G141 grism to acquire slitless spectroscopy over the $1.1-1.7 \mu \mathrm{m}$ range for faint targets not observable from ground-based facilities (Program 12330; PI: J. D. Kirkpatrick). The resulting resolving power, $R \approx 130$, is ideal for the broad characterization of faint sources. Three spectra-those of WISEPC J145018.40+553421.4, WISEPA J173835.53+273258.9, and WISEPA J182831.08+265037.8 - were obtained with this setup. For each target, we first obtained four direct images through the F140W filter in MULTIACCUM mode using the SPARS25 sampling sequence. The telescope was offset slightly between each exposure. We then obtained four dispersed images with the G141 grism using MULTIACCUM mode and a SPARS50 sequence. These dispersed images were acquired at the same positions/dithers as used in the direct images.

Bias levels and dark current were first subtracted from the data images using version 2.3 of the CALWFC3 pipeline. CALWFC3 also flat fields the direct images, but the grism images are flat fielded during the extraction process described below. Spectra were then extracted using the aXe software (Kümmel et al. 2009). Because aXe requires knowledge of the position and brightness of the targets in the field of view, we combined the four direct images using MULTIDRIZZLE (Koekemoer et al. 2002) and the latest Instrument Distortion Coefficient Table (IDCTAB). SExtractor (Bertin \& Arnouts 1996) was then used to produce a catalog of objects in the field. For each cataloged object, 2D subimages centered on the first-order spectra of each object were combined using AXEDRIZZLE to produce a final spectral image. These subimages were used to extract flux-calibrated spectra. Additional details on the data reduction process are discussed in Cushing et al. (2011).

\section{ANALYSIS}

\subsection{Deriving Spectral Types}

The list of spectroscopically confirmed WISE brown dwarfs is given in Table 4. Abbreviated source names ${ }^{36}$ are shown in Column 1; optical spectral types are shown in Column 2; near-infrared types are shown in Column 3; and the source of the spectrum, integration time, telluric corrector star (for ground-based observations), and observation date are shown in Columns 4-7. The optical and near-infrared classifications of these sources are discussed further below.

\subsubsection{Optical Spectral Types}

Keck/LRIS spectra were obtained for seven of our candidates. Reduced spectra from 8000 to $10000 \AA$ are shown in Figure 15,

36 Throughout the rest of the paper, we will abbreviate WISE source names to
the form "WISE hhmm \pm ddmm." Full designations can be found in Table 2 . 
Table 4

Follow-up Spectroscopy of WISE Brown Dwarf Discoveries

\begin{tabular}{|c|c|c|c|c|c|c|}
\hline $\begin{array}{l}\text { Object Name } \\
\text { and } \mathrm{J} 2000 \text { Coordinates } \\
\text { (1) }\end{array}$ & $\begin{array}{l}\text { Opt. Sp. } \\
\text { Type }^{c} \\
\text { (2) }\end{array}$ & $\begin{array}{l}\text { NIR Sp. } \\
\text { Type }^{c} \\
\text { (3) }\end{array}$ & $\begin{array}{c}\text { Spectrograph } \\
\text { (4) }\end{array}$ & $\begin{array}{l}\text { Int. Time } \\
\text { (s) } \\
(5)\end{array}$ & $\begin{array}{l}\text { Tell. Corr. } \\
\text { Star }^{\mathrm{e}} \\
(6)\end{array}$ & $\begin{array}{l}\text { Obs. Date } \\
\text { (UT) } \\
\text { (7) }\end{array}$ \\
\hline WISE J0008-1739 & $\ldots$ & T6 & Keck/NIRSPEC-J & 1200 & HD 13936 & 2010 Dec 18 \\
\hline WISE J0031-3840 & $\ldots$ & L2 pec (blue) & IRTF/SpeX & 720 & HD 4065 & 2010 Nov 17 \\
\hline WISE J0049+0441 & $\ldots$ & L9 & IRTF/SpeX & 1200 & HD 7215 & 2010 Sep 14 \\
\hline WISE J0106+1518 & $\ldots$ & M8 pec & IRTF/SpeX & 960 & HD 7215 & 2010 Sep 14 \\
\hline WISE J0123+4142 & $\ldots$ & $\mathrm{T} 7$ & Keck/NIRSPEC-J & 600 & HD 13936 & 2010 Dec 19 \\
\hline WISE J0138-0322 & $\ldots$ & T3 & IRTF/SpeX & 960 & HD 13936 & 2010 Aug 17 \\
\hline WISE J0148-7202 & $\ldots$ & T9.5 & Magellan/FIRE & 960 & HD 1881 & 2010 Sep 18 \\
\hline WISE J0150+3827 & $\ldots$ & T0 & IRTF/SpeX & 1920 & HD 19600 & 2010 Sep 12 \\
\hline WISE J0206+2640 & $\ldots$ & L9 pec (red) & IRTF/SpeX & 1680 & HD 19600 & 2010 Sep 12 \\
\hline WISE J0221+3842 & $\ldots$ & T6.5 & Palomar/TSpec & 1200 & HD 19600 & 2011 Jan 22 \\
\hline \multirow[t]{2}{*}{ WISE J0223-2932 } & $\ldots$ & $\mathrm{T} 7$ & Keck/NIRSPEC-H & 1200 & HD 25792 & $2010 \mathrm{Jul} 18$ \\
\hline & $\ldots$ & $\mathrm{T} 7.5$ & IRTF/SpeX & 1560 & HD 20423 & 2010 Aug 17 \\
\hline WISE J0226-0211 & $\ldots$ & $\mathrm{T} 7$ & Keck/NIRSPEC-H & 1200 & HD 21875 & 2010 Jul 19 \\
\hline WISE J0305+3954 & $\ldots$ & T6 & Palomar/TSpec & 1200 & HD 19600 & 2011 Jan 22 \\
\hline WISE J0307+2904 & $\ldots$ & T6.5 & IRTF/SpeX & 720 & HD 19600 & 2010 Dec 17 \\
\hline WISE J0313+7807 & $\ldots$ & $\mathrm{T} 8.5$ & Palomar/TSpec & 1800 & HD 210501 & 2010 Nov 16 \\
\hline WISE J0323-6025 & $\ldots$ & $\mathrm{T} 8.5$ & Magellan/FIRE & 1268 & HD 62762 & 2010 Dec 24 \\
\hline WISE J0333-5856 & $\ldots$ & $\mathrm{T} 3$ & SOAR/OSIRIS & 1080 & HD 16636 & 2010 Dec 28 \\
\hline WISE J0410+1502 & $\ldots$ & Y0 & Magellan/FIRE & 600 & HD 18620 & 2010 Nov 18 \\
\hline WISE J0410+1411 & $\ldots$ & T6 & IRTF/SpeX & 600 & HD 25175 & 2010 Dec 18 \\
\hline WISE J0448-1935 & $\ldots$ & T5 pec & IRTF/SpeX & 240 & HD 29433 & 2010 Aug 17 \\
\hline WISE J0458+6434 & $\ldots$ & $\mathrm{T} 8.5$ & $\ldots{ }^{\mathrm{a}}$ & $\ldots^{\mathrm{a}}$ & $\ldots^{\mathrm{a}}$ & $\ldots{ }^{\mathrm{a}}$ \\
\hline WISE J0500-1223 & $\ldots$ & $\mathrm{T} 8$ & IRTF/SpeX & 960 & HD 31743 & 2010 Oct 29 \\
\hline WISE J0513+0608 & $\ldots$ & T6.5 & Palomar/TSpec & 1200 & HD 40686 & 2010 Nov 15 \\
\hline WISE J0525+6739 & $\ldots$ & T6 pec & IRTF/SpeX & 3240 & HD 38831 & 2010 Nov 17 \\
\hline WISE J0528-3308 & $\ldots$ & $\mathrm{T} 7$ pec & Palomar/TSpec & 2400 & HD 38056 & 2010 Nov 16 \\
\hline WISE J0539-1034 & $\ldots$ & T5.5 & Palomar/TSpec & 2400 & HD 38386 & 2011 Jan 22 \\
\hline WISE J0542-1628 & $\ldots$ & T6.5 & Palomar/TSpec & 2400 & HD 21127 & 2010 Nov 16 \\
\hline WISE J0612-4920 & $\ldots$ & $\mathrm{T} 3.5$ & SOAR/OSIRIS & 1080 & HD 48169 & 2010 Dec 28 \\
\hline WISE J0614+3912 & $\ldots$ & T6 & Palomar/TSpec & 1800 & HD 56385 & 2010 Nov 15 \\
\hline WISE J0623-0456 & $\ldots$ & $\mathrm{T} 8$ & IRTF/SpeX & 900 & HD 45137 & 2010 Dec 17 \\
\hline WISE J0625+5646 & $\ldots$ & T6 & Palomar/TSpec & 1200 & HD 38831 & 2011 Jan 22 \\
\hline WISE J0627-1114 & $\ldots$ & T6 & IRTF/SpeX & 720 & HD 56525 & 2010 Dec 18 \\
\hline WISE J0656+4205 & $\ldots$ & $\mathrm{T} 3$ & IRTF/SpeX & 240 & HD 56525 & 2010 Dec 18 \\
\hline WISE J0744+5628 & $\ldots$ & T8 & Palomar/TSpec & 2400 & HD 45105 & 2011 Jan 22 \\
\hline \multirow[t]{2}{*}{ WISE J0750+2725 } & $\ldots$ & T8.5 & Keck/NIRSPEC-J & 1800 & HD 71906 & 2010 Dec 24 \\
\hline & $\ldots$ & $\mathrm{T} 8.5$ & Keck/NIRSPEC-H & 3000 & HD 71906 & 2010 Dec $24 / 25$ \\
\hline WISE J0751-7634 & $\ldots$ & T9 & Magellan/FIRE & 760 & HD 98671 & 2010 Apr 3 \\
\hline WISE J0759-4904 & $\ldots$ & $\mathrm{T} 8$ & Magellan/FIRE & 760 & CPD-44 2691 & 2011 Mar 25 \\
\hline WISE J0819-0335 & $\ldots$ & $\mathrm{T} 4$ & IRTF/SpeX & 360 & HD 65241 & 2010 Dec 18 \\
\hline WISE J0821+1443 & $\ldots$ & T5.5 & IRTF/SpeX & 720 & HD 65241 & 2010 Dec 18 \\
\hline WISE J0836-1859 & $\ldots$ & T8 pec & Magellan/FIRE & 1522 & HD 75159 & 2011 Mar 27 \\
\hline WISE J0857+5604 & $\ldots$ & $\mathrm{T} 8$ & Palomar/TSpec & 2400 & HD 45105 & 2011 Jan 22 \\
\hline WISE J0906+4735 & $\ldots$ & $\mathrm{T} 8$ & Palomar/TSpec & 3600 & HD 82191 & 2010 Jun 4 \\
\hline WISE J0929+0409 & $\ldots$ & T6.5 & Palomar/TSpec & 1200 & HD 121409 & 2011 Jan 22 \\
\hline WISE J0952+1955 & $\ldots$ & T6 & IRTF/SpeX & 1800 & HD 89239 & 2011 Mar 9 \\
\hline WISE J1018-2445 & $\ldots$ & $\mathrm{T} 8$ & Magellan/FIRE & 507 & HD 90738 & 2011 Mar 27 \\
\hline \multirow[t]{3}{*}{ WISE J1019+6529 } & $\ldots$ & T6 & IRTF/SpeX & 1680 & HD 143187 & 2010 May 27 \\
\hline & $\ldots$ & T6 & Palomar/TSpec & 2400 & SAO 15429 & 2010 May 30 \\
\hline & $\mathrm{T}_{\mathrm{o}} 7$ & $\ldots$ & Keck/LRIS & 3000 & HD 151506 & 2010 Jun 18 \\
\hline WISE J1042-3842 & $\ldots$ & $\mathrm{T} 8.5$ & Magellan/FIRE & 1014 & HD 90738 & 2011 Mar 27 \\
\hline WISE J1122+2550 & $\ldots$ & T6 & IRTF/SpeX & 1440 & HD 99966 & 2010 Jul 14 \\
\hline WISE J1150+6302 & $\ldots$ & $\mathrm{T} 8$ & Palomar/TSpec & 2400 & HD 121409 & 2011 Jan 22 \\
\hline WISE J1217+1626 & $\cdots$ & T9 & Palomar/TSpec & 2400 & HD 19600 & 2011 Jan 22 \\
\hline WISE J1311+0122 & $\ldots$ & T9: & Keck/NIRSPEC-H & 900 & HD 71906 & $2010 \operatorname{Dec} 25$ \\
\hline WISE J1311+3629 & $\ldots$ & L5 pec (blue) & IRTF/SpeX & 1200 & HD 109615 & 2011 Jan 26 \\
\hline WISE J1320+6034 & $\ldots$ & T6.5 & IRTF/SpeX & 720 & HD 118214 & $2010 \mathrm{Jul} 2$ \\
\hline WISE J1322-2340 & $\ldots$ & $\mathrm{T} 8$ & IRTF/SpeX & 1920 & HD 114345 & 2010 May 24 \\
\hline
\end{tabular}


Table 4

(Continued)

\begin{tabular}{|c|c|c|c|c|c|c|}
\hline $\begin{array}{l}\text { Object Name } \\
\text { and J } 2000 \text { Coordinates } \\
\text { (1) }\end{array}$ & $\begin{array}{l}\text { Opt. Sp. } \\
\text { Type }^{c} \\
\text { (2) }\end{array}$ & $\begin{array}{l}\text { NIR Sp. } \\
\text { Type }^{c} \\
\text { (3) }\end{array}$ & $\begin{array}{c}\text { Spectrograph } \\
\text { (4) }\end{array}$ & $\begin{array}{l}\text { Int. Time } \\
\text { (s) } \\
(5)\end{array}$ & $\begin{array}{l}\text { Tell. Corr. } \\
\text { Star }^{\mathrm{e}} \\
(6)\end{array}$ & $\begin{array}{l}\text { Obs. Date } \\
\text { (UT) } \\
(7)\end{array}$ \\
\hline \multirow[t]{2}{*}{ WISE J1457+5815 } & $\ldots$ & $\mathrm{T} 7$ & IRTF/SpeX & 960 & HD 143187 & $2010 \mathrm{Jul} 14$ \\
\hline & $\mathrm{T}_{0} 8$ & $\ldots$ & Keck/LRIS & 3600 & HD 238493 & $2010 \mathrm{Jul} 17$ \\
\hline \multirow[t]{2}{*}{ WISE J1506+7027 } & $\ldots$ & T6 & Keck/NIRSPEC-H & 960 & HD $25792^{\mathrm{d}}$ & 2010 Oct 20 \\
\hline & $\ldots$ & $\mathrm{T} 6$ & Palomar/TSpec & 960 & HD 145454 & 2011 Jan 22 \\
\hline WISE J1519+7009 & $\ldots$ & T8 & Palomar/TSpec & 2400 & HD 145454 & 2010 Jun 4 \\
\hline WISE J1541-2250 & $\ldots$ & Y0 & Magellan/FIRE & 1522 & HD 130755 & 2011 Mar 27 \\
\hline WISE J1612-3420 & $\ldots$ & T6.5 & Keck/NIRSPEC-H & 1200 & HD 152384 & 2010 Jul 19 \\
\hline WISE J1614+1739 & $\ldots$ & T9 & Magellan/FIRE & 600 & HD 98671 & 2010 Apr 3 \\
\hline \multirow[t]{2}{*}{ WISE J1617+1807 } & $\ldots$ & $\mathrm{T} 8$ & $\ldots{ }^{b}$ & $\ldots{ }^{b}$ & $\ldots b$ & $\ldots \mathrm{b}$ \\
\hline & $\mathrm{T}_{0} 8$ & $\ldots$ & Keck/LRIS & 3600 & BD+18 3241 & $2010 \mathrm{Jul} 17$ \\
\hline WISE J1622-0959 & $\ldots$ & T6 & IRTF/SpeX & 1200 & HD 148968 & 2010 Apr 23 \\
\hline \multirow[t]{2}{*}{ WISE J1627+3255 } & $\ldots$ & T6 & Keck/NIRSPEC-H & 2400 & HD 145647 & 2010 Feb 24 \\
\hline & $\ldots$ & $\mathrm{T} 6$ & IRTF/SpeX & 4080 & HD 145647 & $2010 \mathrm{Feb} 28$ \\
\hline WISE J1647+5632 & $\ldots$ & L9 pec (red) & IRTF/SpeX & 960 & HD 179933 & 2010 Aug 17 \\
\hline \multirow{2}{*}{ WISE J1653+4444 } & $\ldots$ & $\mathrm{T} 8$ & IRTF/SpeX & 1440 & HD 143187 & 2010 Apr 21 \\
\hline & $\mathrm{T}_{0} 8$ & $\ldots$ & Keck/LRIS & 2400 & HD 159518 & $2010 \mathrm{Jul} 17$ \\
\hline WISE J1711+3500 & $\ldots$ & $\mathrm{T} 8$ & IRTF/SpeX & 1440 & HD 165029 & $2010 \mathrm{Jul} 13$ \\
\hline \multirow[t]{2}{*}{ WISE J1717+6129 } & $\ldots$ & $\mathrm{T} 8$ & Keck/NIRSPEC-J & 1200 & HD 179933 & 2010 Jul 19 \\
\hline & $\ldots$ & $\mathrm{T} 8$ & Keck/NIRSPEC-H & 1200 & HD 179933 & $2010 \mathrm{Jul} 19$ \\
\hline WISE J1728+5716 & $\ldots$ & $\mathrm{T} 6$ & IRTF/SpeX & 3120 & HD 143187 & 2010 Apr 21 \\
\hline WISE J1738+2732 & $\ldots$ & Y0 & $H S T / \mathrm{WFC} 3$ & 2012 & $\ldots$ & 2011 May 12 \\
\hline \multirow[t]{2}{*}{ WISE J1741+2553 } & $\ldots$ & $\mathrm{T} 9$ & Magellan/FIRE & 1800 & HD 98671 & 2010 Apr 3 \\
\hline & $\mathrm{T}_{0} 9$ & $\ldots$ & Keck/LRIS & 3900 & HD 335701 & 2010 Jun 18 \\
\hline WISE J1804+3117 & $\ldots$ & T9.5: & Keck/NIRSPEC-H & 1200 & HD 171623 & 2010 Jul 19 \\
\hline WISE J1812+2721 & $\ldots$ & T8.5: & $\ldots{ }^{b}$ & $\ldots{ }^{b}$ & $\ldots{ }^{b}$ & $\ldots{ }^{b}$ \\
\hline WISE J1828+2650 & $\ldots$ & $>\mathrm{Y} 0$ & $H S T / \mathrm{WFC} 3$ & 2012 & $\ldots$ & 2011 May 9 \\
\hline WISE J1830+4542 & $\ldots$ & L9 & IRTF/SpeX & 1920 & HD 178207 & 2010 Sep 12 \\
\hline \multirow[t]{2}{*}{ WISE J1841+7000 } & $\ldots$ & $\mathrm{T} 5$ & Palomar/TSpec & 2400 & HD 179933 & 2010 Jun 4 \\
\hline & $\mathrm{T}_{0} 5$ & $\ldots$ & Keck/LRIS & 3600 & $\mathrm{BD}+701059$ & $2010 \mathrm{Jul} 17$ \\
\hline WISE J1852+3537 & $\ldots$ & $\mathrm{T} 7$ & IRTF/SpeX & 1680 & HD 174567 & 2010 May 25 \\
\hline WISE J1906+4508 & $\ldots$ & $\mathrm{T} 6$ & IRTF/SpeX & 1200 & HD 174567 & 2010 Nov 17 \\
\hline WISE J1952+7240 & $\ldots$ & $\mathrm{T} 4$ & Palomar/TSpec & 1200 & HD 18187 & 2010 Nov 16 \\
\hline WISE J1959-3338 & $\ldots$ & $\mathrm{T} 8$ & Palomar/TSpec & 3600 & HD 194272 & 2010 Jun 4 \\
\hline WISE J2018-7423 & $\ldots$ & $\mathrm{T} 7$ & $\ldots \mathrm{b}^{\mathrm{t}}$ & $\ldots{ }^{b}$ & & \\
\hline \multirow[t]{2}{*}{ WISE J2056+1459 } & $\ldots$ & Y0 & Keck/NIRSPEC-J & 2400 & HD 198070 & 2010 Oct 21 \\
\hline & $\ldots$ & Y0 & Keck/NIRSPEC-H & 1800 & HD 198069 & 2010 Nov 22 \\
\hline WISE J2134-7137 & $\ldots$ & T9 pec & Magellan/FIRE & 600 & HD 223296 & 2010 Sep 18 \\
\hline WISE J2157+2659 & $\ldots$ & T7 & Palomar/TSpec & 1200 & HD 208108 & 2010 Sep 29 \\
\hline WISE J2209-2734 & $\ldots$ & $\mathrm{T} 7$ & Keck/NIRSPEC-H & 600 & HD 212643 & $2010 \mathrm{Jul} 18$ \\
\hline WISE J2213+0911 & $\ldots$ & $\mathrm{T} 7$ & IRTF/SpeX & 2400 & HD 210501 & 2010 Aug 4 \\
\hline \multirow[t]{3}{*}{ WISE J2226+0440 } & $\ldots$ & $\mathrm{T} 8.5$ & Keck/NIRSPEC-J & 1200 & HD 190807 & $2010 \mathrm{Jul} 18$ \\
\hline & $\ldots$ & $\mathrm{T} 8$ & Keck/NIRSPEC-H & 1200 & HD 190807 & $2010 \mathrm{Jul} 18$ \\
\hline & $\ldots$ & $\mathrm{T} 8$ & IRTF/SpeX & 1920 & HD 210501 & 2010 Aug 4 \\
\hline WISE J2237-0614 & $\ldots$ & $\mathrm{T} 5$ & IRTF/SpeX & 1200 & HD 219833 & $2010 \mathrm{Jul} 14$ \\
\hline WISE J2239+1617 & $\ldots$ & $\mathrm{T} 3$ & IRTF/SpeX & 1200 & HD 210501 & 2010 Aug 17 \\
\hline \multirow[t]{2}{*}{ WISE J2255-3118 } & $\ldots$ & $\mathrm{T} 8$ & Keck/NIRSPEC-H & 1200 & HD 202941 & $2010 \mathrm{Jul} 19$ \\
\hline & $\ldots$ & $\mathrm{T} 8$ & IRTF/SpeX & 2400 & HD 202025 & 2010 Sep 10 \\
\hline WISE J2313-8037 & $\ldots$ & $\mathrm{T} 8$ & $\ldots \mathrm{b}^{\mathrm{b}}$ & $\ldots{ }^{b}$ & $\ldots{ }^{b}$ & $\ldots \mathrm{b}^{\mathrm{b}}$ \\
\hline WISE J2319-1844 & $\ldots$ & $\mathrm{T} 7.5$ & IRTF/SpeX & 2880 & HD 222332 & 2010 Aug 17 \\
\hline WISE J2325-4105 & $\ldots$ & T9 pec & Magellan/FIRE & 960 & HD 221805 & 2010 Sep 18 \\
\hline WISE J2327-2730 & $\ldots$ & L9 & IRTF/SpeX & 1200 & HD 219290 & 2010 Sep 12 \\
\hline \multirow[t]{2}{*}{ WISE J2340-0745 } & $\ldots$ & $\mathrm{T} 7$ & IRTF/SpeX & 960 & HD 219833 & $2010 \mathrm{Jul} 14$ \\
\hline & $\mathrm{T}_{0} 7$ & $\ldots$ & Keck/LRIS & 2400 & HD 1920 & 2010 Jul 17 \\
\hline WISE J2343-7418 & $\ldots$ & T6 & Magellan/FIRE & 634 & HD 223296 & 2010 Dec 24 \\
\hline WISE J2344+1034 & $\ldots$ & $\mathrm{T} 9$ & Keck/NIRSPEC-J & 1200 & HD 198070 & 2010 Oct 21 \\
\hline WISE J2348-1028 & $\ldots$ & $\mathrm{T} 7$ & IRTF/SpeX & 960 & HD 219833 & $2010 \mathrm{Jul} 14$ \\
\hline WISE J2359-7335 & $\ldots$ & $\mathrm{T} 5.5$ & $\ldots{ }^{b}$ & $\ldots{ }^{b}$ & $\ldots{ }^{b}$ & $\ldots{ }^{b}$ \\
\hline
\end{tabular}

Notes.

a See Mainzer et al. (2011) for details on spectroscopic follow-up.

${ }^{\mathrm{b}}$ See Burgasser et al. (2011a) for details on spectroscopic follow-up. A newer spectrum of this object from Magellan/FIRE supports a type of T6.5 rather than T5.5 as originally assigned by Burgasser et al. (2011a).

c Spectral types are accurate to \pm 0.5 type unless indicated by a ":" symbol to indicate a more uncertain assignment.

${ }^{\mathrm{d}}$ Due to closing of the telescope due to fog, no A0 V star was taken on this night so an A0 V observation from an earlier run on 2010 July 18 was used.

${ }^{\mathrm{e}}$ Telluric corrector stars for optical spectra are G0 dwarfs; those for near-infrared spectra are A0 dwarfs. 


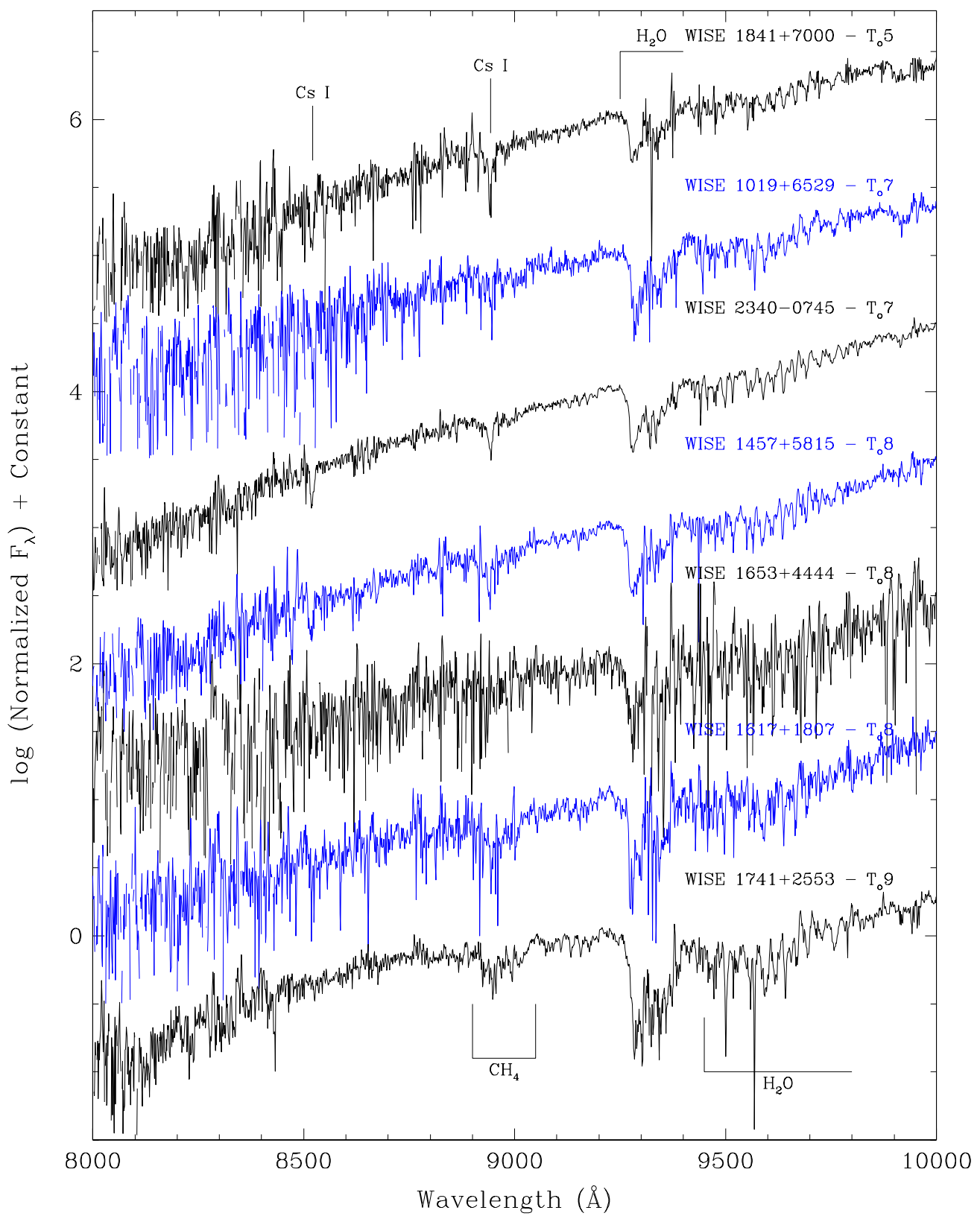

Figure 15. Spectra from 8000 to $10000 \AA$ for seven WISE brown dwarf discoveries. Data have been corrected for telluric absorption and prominent spectral features are marked. All spectra have been normalized at $9200 \AA$ and an integral offset added to the $y$-axis values to separate the spectra vertically.

(A color version of this figure is available in the online journal.)

all of which have been corrected for telluric absorption over the regions $6867-7000 \AA$ (the Fraunhofer $\mathrm{B}$ band, caused by $\mathrm{O}_{2}$ absorption), 7594-7685 $\AA$ (the Fraunhofer A band, again caused by $\mathrm{O}_{2}$ ), and 7186-7273, 8162-8282, and $\sim 8950-9650 \AA$ (all caused by $\mathrm{H}_{2} \mathrm{O}$ absorption). These spectra show the hallmarks of T dwarf optical spectra: strong $\mathrm{H}_{2} \mathrm{O}$ absorption with a bandhead at $9250 \AA$, along with Cs I absorption at 8521 and $8943 \AA$ in the earlier objects and $\mathrm{CH}_{4}$ absorption between 8800 and $9200 \AA$ in the later objects.

By-eye comparisons to the T dwarf optical spectral standards of Burgasser et al. (2003a) show that these spectra range in type from $T_{0} 5$ for WISE $1841+7000$ to later than $T_{0} 8$ for WISE $1741+2553$. (The "o" subscript is used to denote spectral types assigned based on optical spectra.) This latter spectrum is unusual in that it has stronger $8800-9200 \AA \mathrm{CH}_{4}$, stronger $9250-9400 \AA \mathrm{H}_{2} \mathrm{O}$ bands of the $3\left(v_{1}, v_{3}\right)$ transition, and stronger
9450-9800 $\AA \mathrm{H}_{2} \mathrm{O}$ bands of the $2\left(v_{1}, v_{3}\right)+2\left(v_{2}\right)$ transition than the latest $\mathrm{T}$ dwarf optical standard, the $\mathrm{T}_{0} 8$ dwarf 2MASS J04151954-0935066 (Burgasser et al. 2003a). We therefore propose that WISE $1741+2553$ be the spectral standard for a newly adopted $\mathrm{T}_{\mathrm{o}} 9$ spectral class. Figure 16 illustrates the entire sequence of $\mathrm{T}$ dwarf optical standards from Burgasser et al. (2003a) appended with the proposed $\mathrm{T}_{0} 0$ standard SDSSp J083717.22-000018.3 from Kirkpatrick (2008) and this newly proposed $\mathrm{T}_{\mathrm{o}} 9$ standard.

Further evidence in support of a new optical standard is shown in Figure 17. Shown here is a comparison of the spectrum of WISE $1741+2553$ with our LRIS spectrum of the bright, late-T dwarf UGPS J072227.51-054031.2, which Cushing et al. (2011) have proposed as the infrared spectral standard for type T9. As our comparison shows, the $\mathrm{CH}_{4}$ and $\mathrm{H}_{2} \mathrm{O}$ depths are very similar between these two objects and both 


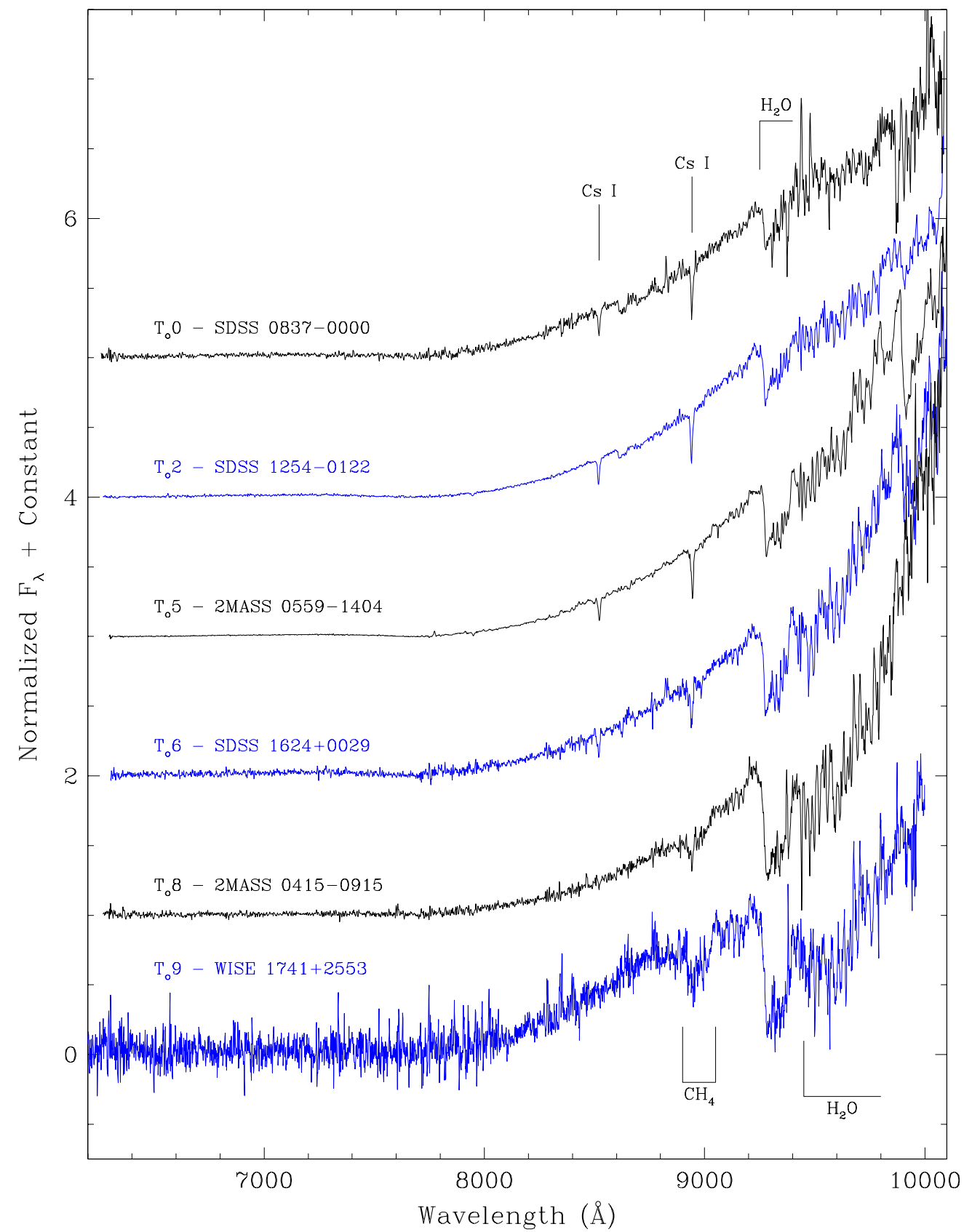

Figure 16. Spectra from 6200 to $10000 \AA$ for the optical spectral standards from Burgasser et al. (2006b) supplemented with the $\mathrm{T}_{0} 0$ standard from Kirkpatrick et al. (2010) and the $\mathrm{T}_{0} 9$ standard proposed here. Data have been corrected for telluric absorption and prominent spectral features are marked. All spectra have been normalized at $9200 \AA$ and an integral offset added to the $y$-axis values to separate the spectra vertically.

(A color version of this figure is available in the online journal.)

are distinctly different from the $\mathrm{T}_{\mathrm{o}} 8$ standard. Thus, identifying WISE $1741+2553$ as the new $\mathrm{T}_{0} 9$ anchor point would help link the optical and near-infrared sequences, especially since WISE $1741+2553$ is also classified as a T9 on the near-infrared scheme. See further discussion in the Appendix.

\subsubsection{Near-infrared Spectral Types}

All of the confirmed brown dwarfs listed in Table 4 have near-infrared follow-up spectra. These spectra were classified using the near-infrared $\mathrm{M}$ and $\mathrm{L}$ sequences of Kirkpatrick et al. (2010) and the near-infrared T0-to-T8 dwarf sequence of Burgasser et al. (2006b). Cushing et al. (2011) have extended classifications to $\mathrm{T} 9$ and $\mathrm{Y} 0$ and have reclassified previously discovered $>\mathrm{T} 8$ dwarfs from the literature on this system. This extension of the classification system uses UGPS $0722-0540$ as the near-infrared T9 standard and WISE $1738+2732$ as the Y0 standard.

Assignment of spectral types was done by overplotting spectra of these standards onto the candidate spectra and determining by-eye which standard provided the best match. In some cases two adjacent standards, such as T7 and T8, provided an equally good match, so the candidate spectrum was assigned an intermediate type, in this case, of T7.5. For L dwarfs, the comparison was done at $J$ band and, following the prescription discussed in Kirkpatrick et al. (2010), any anomalies at the $H$ 


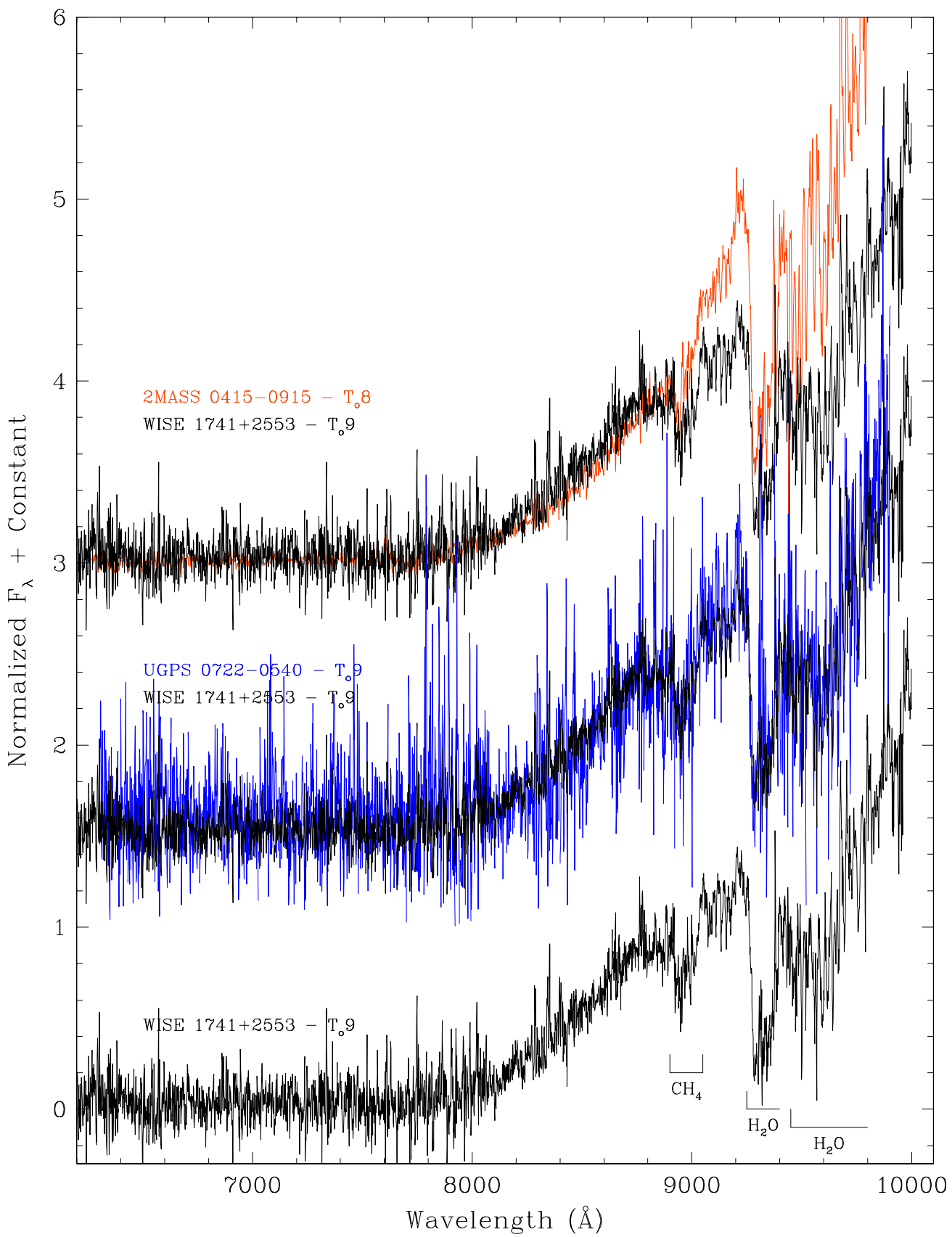

Figure 17. Spectra from 6200 to $10000 \AA$ for three late-T dwarfs: the $T_{0} 8$ standard $2 \mathrm{MASS}$ J04151954-0935066 (orange red), the $\mathrm{T}_{0} 9$ standard WISE $1741+2553$ (black), and the $\mathrm{T}_{0} 9$ dwarf UGPS J072227.51-054031.2 (blue violet). Data have been corrected for telluric absorption and prominent spectral features are marked. Spectra have been normalized at $8800 \AA$ and offsets in increments of 1.5 added to the $y$-axis values to separate the spectra vertically except where overplotting was intended.

(A color version of this figure is available in the online journal.)

and $K$ bands were noted. Spectra that did not match any of the standards well are marked with a "pec" suffix to indicate that they are peculiar. As a further example, an object that best fit the L9 spectral standard at the $J$ band but failed to provide a good match to the L9 standard at longer wavelengths because it was considerably redder than the standard was assigned a type of "L9 pec (red)." See Kirkpatrick et al. (2010) for examples of similar classifications.

In Figures 18-25, we show the near-infrared spectra for each of our sources. Because of the narrow wavelength ranges covered by the Keck/NIRSPEC and SOAR/OSIRIS spectra, those data are plotted separately in Figures 26 and 27.

\subsubsection{Discussion}

Near-infrared spectral types (and optical spectral types, for those with Keck/LRIS spectra) are listed in Table 4 for all WISE brown dwarf discoveries. For objects with near-infrared spectral types of T0 or later, Figure 28 shows the number of newly discovered objects per spectral type bin compared to the number of objects previously published. Whereas there were 16 objects known previously with types of T8 or later (Burgasser et al. 2002; Tinney et al. 2005; Looper et al. 2007; Warren et al. 2007; Delorme et al. 2008, 2010; Burningham et al. 2008, 2009, 2010b, 2011a; Goldman et al. 2010; Lucas et al. 2010), the tally 


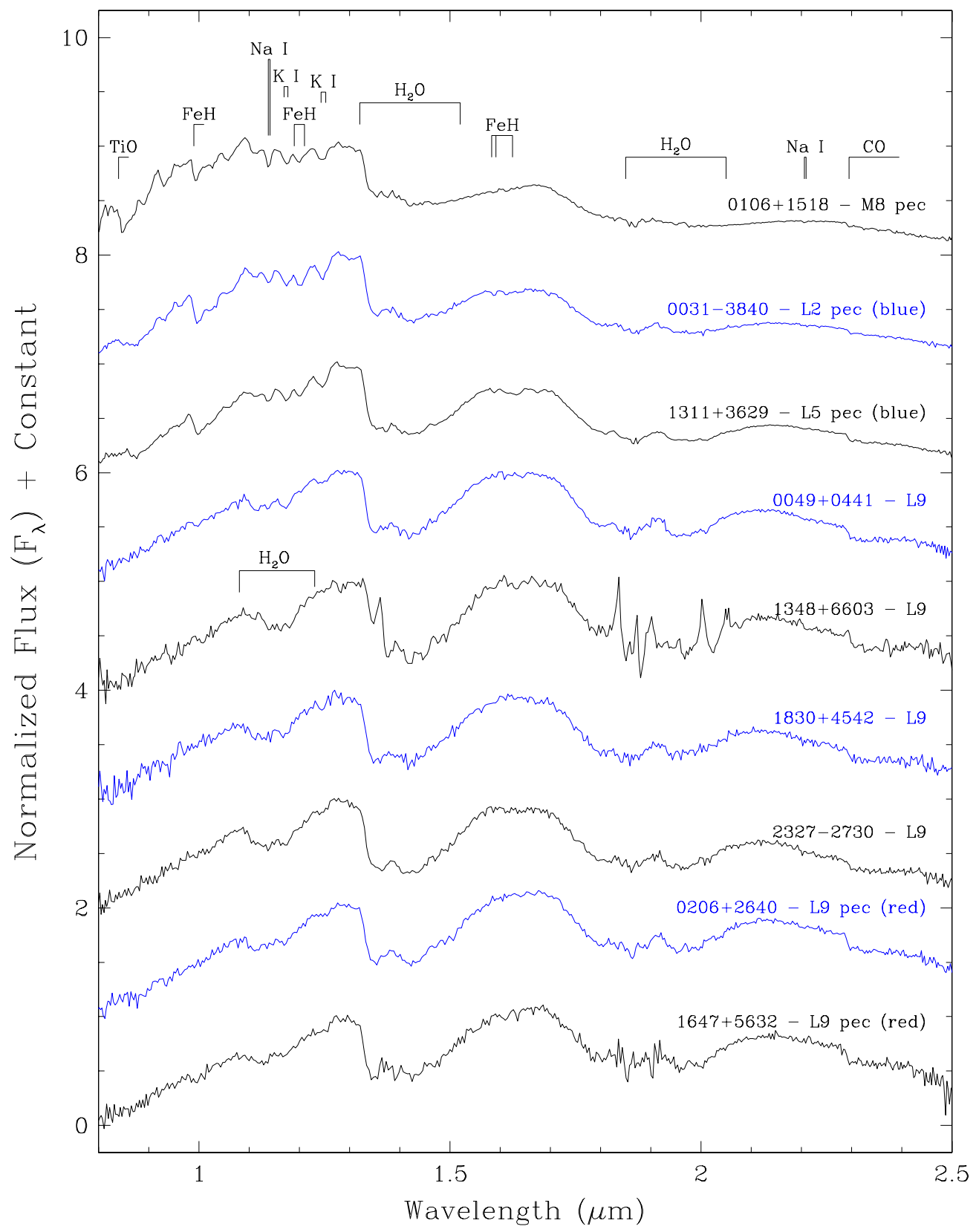

Figure 18. Near-infrared spectra of confirmed WISE brown dwarfs with spectral types earlier than T0. Spectra have been normalized to one around $1.28 \mu \mathrm{m}$ and integral offsets have been added to the $y$-axis values to separate the spectra vertically. Prominent spectral features are marked.

(A color version of this figure is available in the online journal.)

now stands at 58 once our objects are added. WISE has already identified seventeen new objects with types equal to or later than the T9 UGPS J072227.51-054031.2, the previous record holder for latest measured spectral type, and six of these belong to the Y dwarf class (Cushing et al. 2011).

Figures 1-3 and 5-14, discussed previously, show the locations of these newly discovered WISE brown dwarfs (black symbols) in color space. The T9, T9.5, and early-Y dwarfs continue the trend toward redder $W 1-W 2, \operatorname{ch} 1-\operatorname{ch} 2, J-W 2$, $H-W 2, J-\operatorname{ch} 2$, and $H-\operatorname{ch} 2$ colors, with the reddest object being the $>$ Y0 dwarf WISE $1828+2650(J-W 2=9.39 \pm$ $0.35 \mathrm{mag} ; J$ on the MKO filter system). The blueward trend in $J-H$ color seen for later T dwarfs, however, begins to reverse near a spectral type of $\mathrm{Y} 0$. In particular, the $J-H$ color of WISE $1828+1650$ is dramatically redder $(J-H=0.72 \pm 0.42$ mag; MKO filter system) than any of the late-T or Y0 dwarfs, the latter of which show a large scatter in $J-H$ colors themselves. Cushing et al. (2011) explore the trend of $J-H$ colors in more detail and show that the synthetic photometry derived from our observed spectra generally agree with photometry measured from direct imaging. Given the large spread in $J-H$ color observed for the six $\mathrm{Y}$ dwarfs already identified, $J H K_{s}$ colors alone cannot be used to confirm or deny objects as cold as Y dwarfs.

\subsection{Distances and Proper Motions}

Distances to the new WISE brown dwarf discoveries can be estimated based on their $W 2$ magnitudes and measured spectral types. First, however, the relation between absolute W2 magnitude and spectral type needs to be established using objects with measured trigonometric parallaxes and WISE W2 


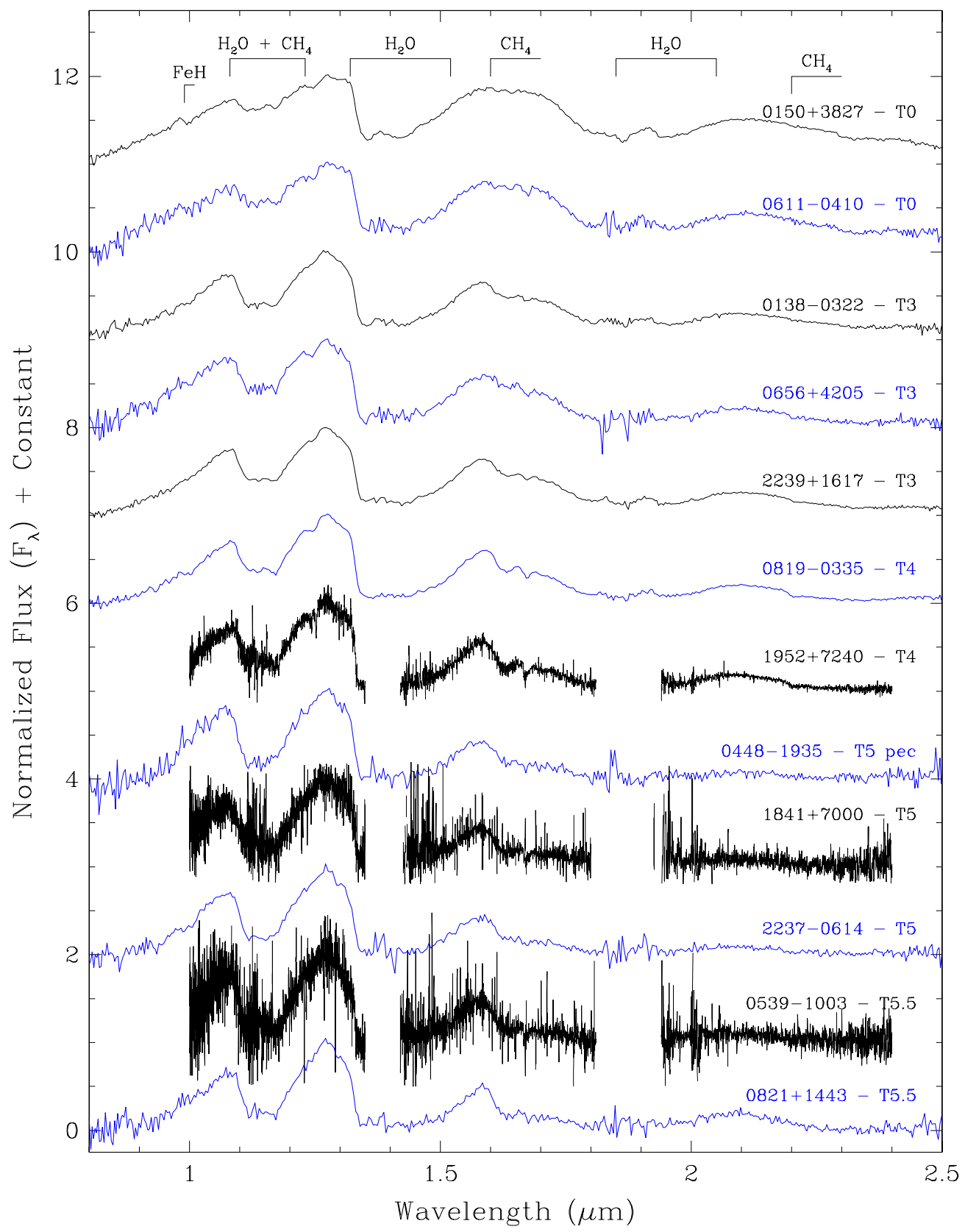

Figure 19. Near-infrared spectra of confirmed WISE brown dwarfs with spectral types from T0 to T5.5. Spectra have been normalized to one around $1.28 \mu \mathrm{m}$ and integral offsets have been added to the $y$-axis values to separate the spectra vertically. For some spectra, noisy data in the depths of the telluric water bands are not plotted. Prominent spectral features are marked.

(A color version of this figure is available in the online journal.)

detections. Figure 29 shows the trend of absolute $W 2$ magnitude as a function of near-infrared spectral type for previously published objects whose measured parallaxes are at least three times the measurement error (Table 5). A third-order leastsquares relation, weighted by the errors on the $M_{W 2}$ values, is shown by the black curve in Figure 29. For this fit, objects known to be binary (red points) have been omitted. The resulting relation is

$$
\begin{aligned}
M_{W 2}= & 9.692+0.3602(\text { Type })-0.02660(\text { Type })^{2} \\
& +0.001020(\text { Type })^{3}
\end{aligned}
$$

where Type is the near-infrared spectral type on the system where $L 0=0, L 5=5, T 0=10, T 5=15$, and $Y 0=20$.
Using this relation, we have estimated distances to our WISE discoveries. These are given in Column 2 of Table 6. (The distance to the lone M dwarf, WISE 0106+1518, was estimated using 2MASS magnitudes and the near-infrared absolute magnitudes listed in Table 3 of Kirkpatrick \& McCarthy 1994.) These distance estimates for the late-T dwarfs and Y dwarfs are shown graphically in Figure 30. Also shown in the figure are previously published late-T dwarfs from other surveys. WISE has sufficient sensitivity to detect the latest $\mathrm{T}$ dwarfs out to $15-20 \mathrm{pc}$ and because of its all-sky coverage can complete the census of the solar neighborhood for these objects. As the figure shows, 12 of our objects have estimated distances placing them within $10 \mathrm{pc}$ of the Sun, and 2 of these have estimates placing them within $5 \mathrm{pc}$. It should also be noted that the fitted 


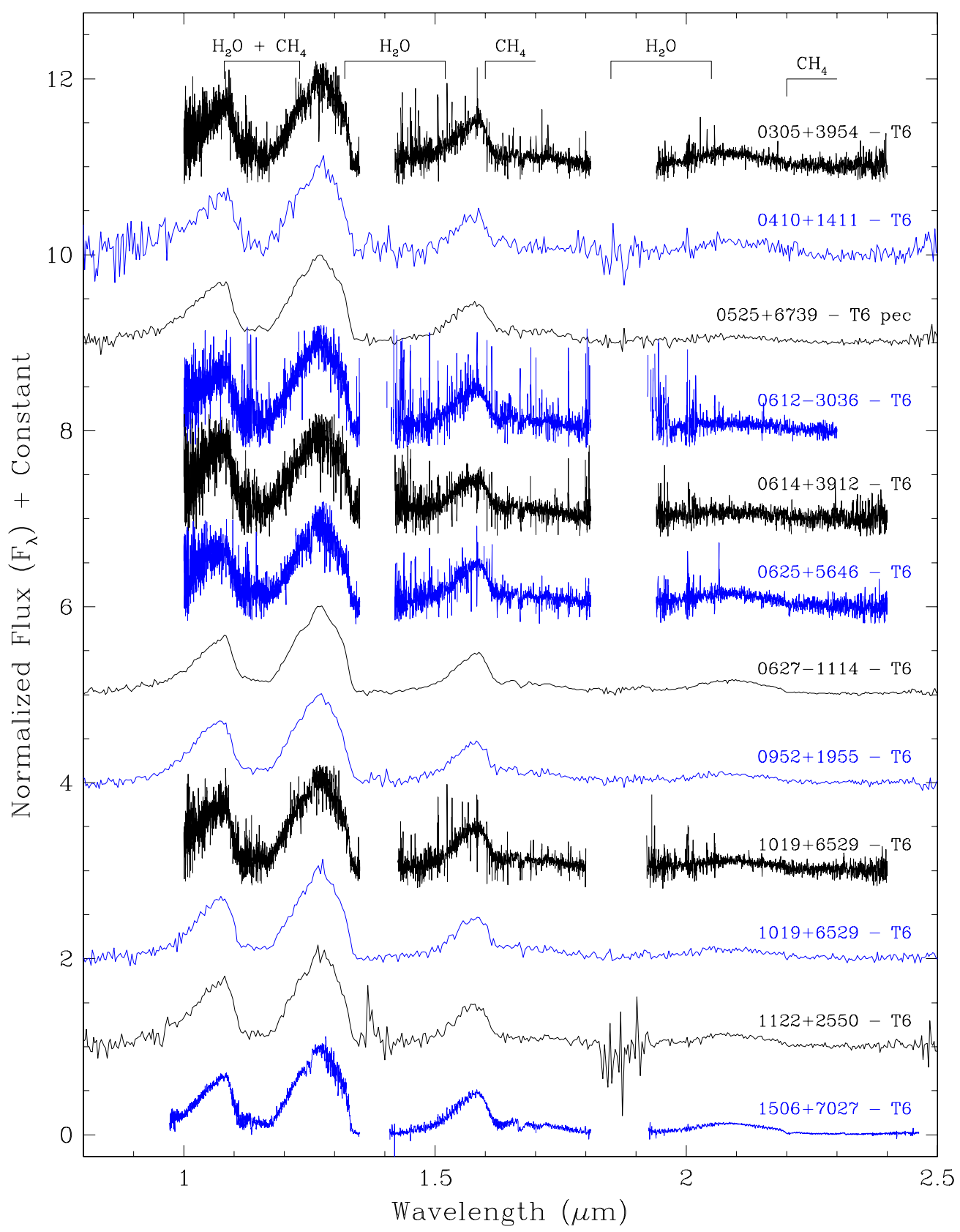

Figure 20. Near-infrared spectra of confirmed WISE brown dwarfs with spectral types of T6. (Additional T6 dwarfs are shown in Figure 21.) Spectra have been normalized to one around $1.28 \mu \mathrm{m}$ and integral offsets have been added to the $y$-axis values to separate the spectra vertically. For some spectra, noisy data in the depths of the telluric water bands are not plotted. Prominent spectral features are marked.

(A color version of this figure is available in the online journal.)

relation shown in Figure 29 may lead to overestimated distances for objects at the latest types because the relation falls above all four of the previously published $\mathrm{T} 8.5$ and $\mathrm{T} 9 \mathrm{dwarfs}$ on that plot. Furthermore, the extrapolation of this relation to even later Y dwarf types may lead to even more discrepant distance overestimates, as discussed further in the caption to Figure 29. Measuring trigonometric parallaxes for more of these latest $T$ dwarfs and early-Y dwarfs will be an important, early step in characterizing the physical nature of these objects.

Because these objects should all lie very close to the Sun, their observed parallaxes will be large. Thanks to its survey strategy, WISE performed its two passes of the sky with observations always near $90^{\circ}$ solar elongation, thus capitalizing on the maximum parallactic angle at both epochs. (For objects observed during the final $\sim 2$ weeks of WISE operations, three epochs of WISE data are available.) Objects will, of course, also show displacements due to proper motion, so observations at other epochs and/or from other surveys are necessary to disentangle the two effects. Hence, ancillary astrometry from 2MASS and SDSS and our own follow-up observations from the ground and from space (Spitzer and HST) are invaluable. Currently available astrometric data points ${ }^{37}$ are shown in Columns 3-8 of Table 6 .

\footnotetext{
37 Future papers will include astrometry taken from our various ground-based imaging campaigns, once data over a longer time baseline have been acquired.
} 


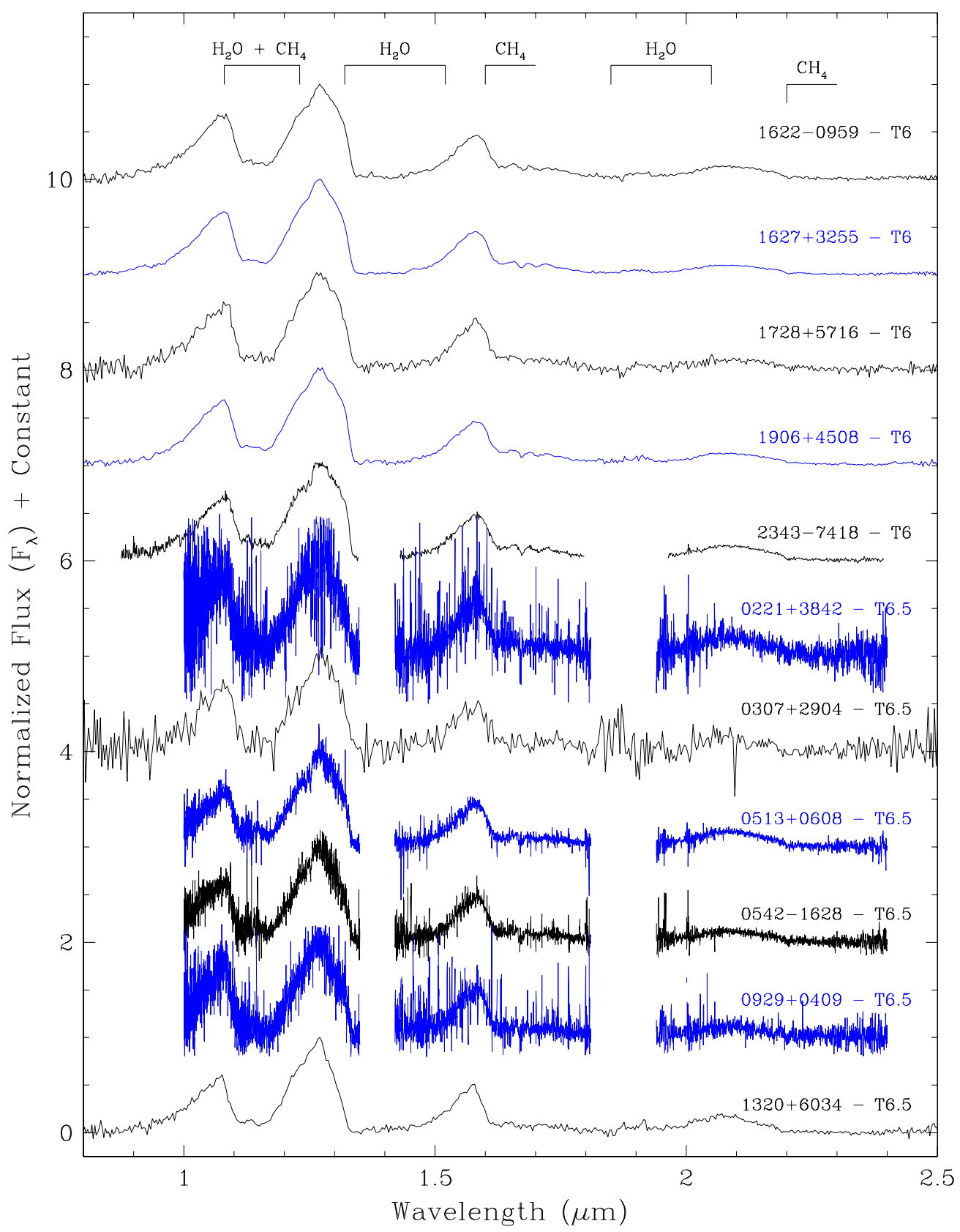

Figure 21. Near-infrared spectra of confirmed WISE brown dwarfs with spectral types from T6 (continued) to T6.5. Spectra have been normalized to one around $1.28 \mu \mathrm{m}$ and integral offsets have been added to the $y$-axis values to separate the spectra vertically. For some spectra, noisy data in the depths of the telluric water bands are not plotted. Prominent spectral features are marked.

(A color version of this figure is available in the online journal.)

It should be noted here that positions of objects in the WISE preliminary data release may be offset from their true positions by many times the quoted positional uncertainty. Approximately $20 \%$ of the sources fainter than $W 1 \approx 14.5 \mathrm{mag}$ in the Preliminary Release Source Catalog suffer from a pipeline coding error that biases the reported position by $\sim 0.2-1.0$ arcsec in the declination direction while an increasingly smaller fraction of the sources suffer this effect to magnitudes as bright as $W 1 \approx 13.0 \mathrm{mag}$. The Cautionary Notes section of the WISE Preliminary Release Explanatory Supplement describes the origin and nature of this effect in detail. For this paper, we have rerun the WISE images for our sources through a version of the WISE pipeline that eliminates this source of sys- tematic error, and we list those remeasured positions in Table 6. This version of the pipeline is essentially the same one used to process data for the WISE Final Data Release.

Astrometric fits were made to the multiple observations of each source. These fits solved for five parameters: initial (time $=t_{i}$ ) positional offsets of $\Delta \alpha$ and $\Delta \delta$ in right ascension $(\alpha)$ and in declination $(\delta)$, the right ascension component of proper motion $\left(\mu_{\alpha}=(\cos \delta) d \alpha / d t\right)$, the declination component of proper motion $\left(\mu_{\delta}=d \delta / d t\right)$, and the parallax $\left(\pi_{\text {trig }}\right)$. For all but four sources, the data were not sufficient to find an accurate distance, so the distance was forced to equal the spectrophotometric estimate, and the fit only solved for the first four parameters; the four sources with a preliminary parallax 


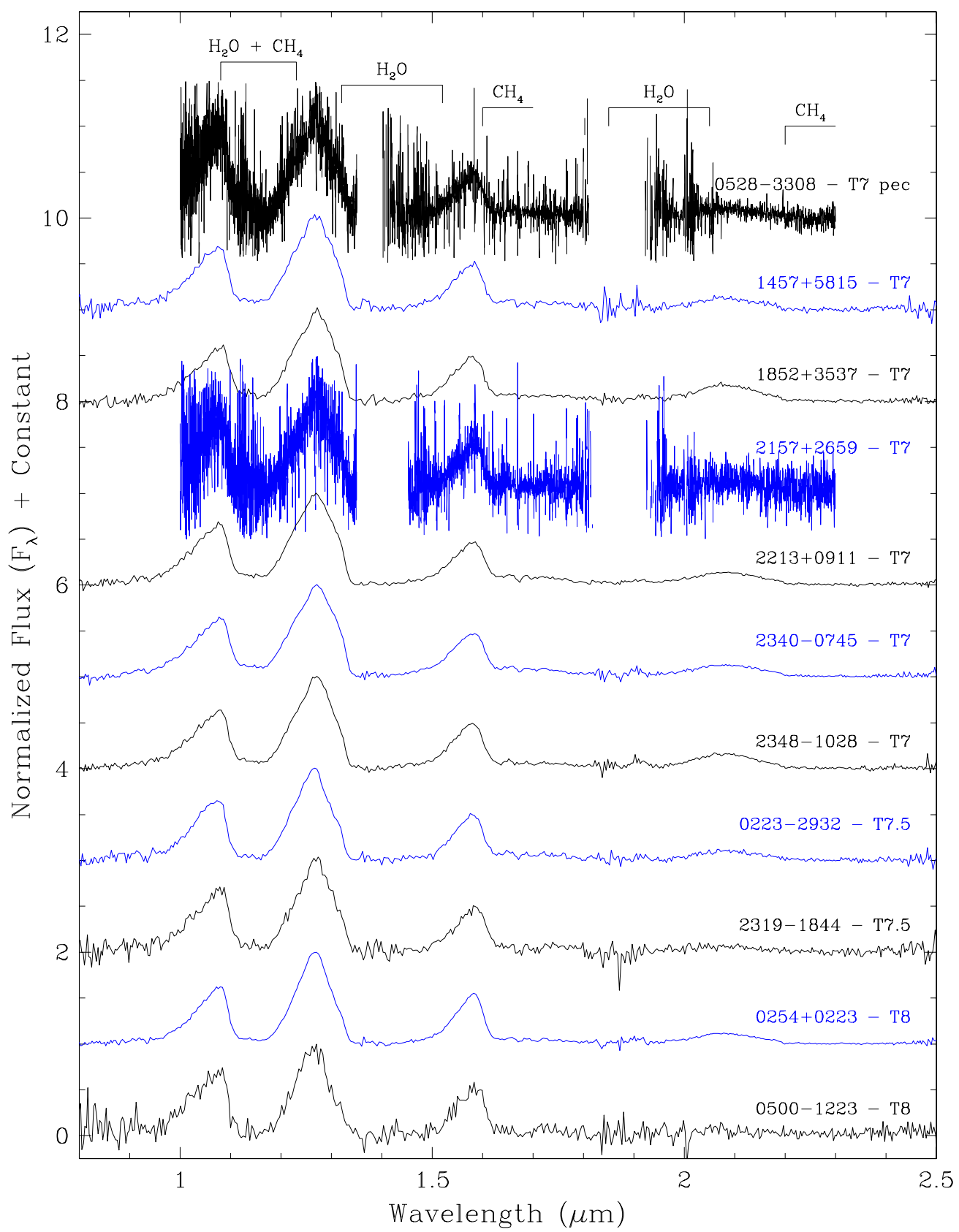

Figure 22. Near-infrared spectra of confirmed WISE brown dwarfs with spectral types from T7 to T8. (Additional T8 dwarfs are shown in Figures 23 and 24 .) Spectra have been normalized to one around $1.28 \mu \mathrm{m}$ and integral offsets have been added to the $y$-axis values to separate the spectra vertically. For some spectra, noisy data in the depths of the telluric water bands are not plotted. Prominent spectral features are marked.

(A color version of this figure is available in the online journal.)

measurement are listed in Table 7 and are discussed individually in the Appendix. The equations used are

$$
\begin{gathered}
\cos \delta_{1}\left(\alpha_{i}-\alpha_{1}\right)=\Delta \alpha+\mu_{\alpha}\left(t_{i}-t_{1}\right)+\pi_{\text {trig }} \vec{R}_{i} \cdot \hat{W}, \text { and } \\
\delta_{i}-\delta_{1}=\Delta \delta+\mu_{\delta}\left(t_{i}-t_{1}\right)-\pi_{\text {trig }} \vec{R}_{i} \cdot \hat{N} .
\end{gathered}
$$

The subscript $i$ refers to the individual astrometric measurements, where $t_{i}$ is the observation time in years, and $R_{i}$ is the vector position of the observer relative to the Sun in celestial coordinates and astronomical units. $\hat{N}$ and $\hat{W}$ are unit vectors pointing north and west from the position of the source. $R_{i}$ is the position of the Earth for 2MASS, SDSS, WISE, and HST observations; for Spitzer observations, $R_{i}$ is the position of the spacecraft. The observed positional difference on the left-hand side is in arcseconds, the parameters $\Delta \alpha$ and $\Delta \delta$ are in arcseconds, the proper motion $\mu_{\alpha}$ and $\mu_{\delta}$ are in $\operatorname{arcsec} \mathrm{yr}^{-1}$, and the parallax $\pi_{\text {trig }}$ is in arcsec.

These equations are solved using standard weighted leastsquares techniques, which also provide the uncertainties in the parameters. These uncertainties come from propagating the uncertainties in the input. The $\chi^{2}$ and number of degrees of freedom are also given and can be used to assess the quality of the fit. The resulting proper motions in R.A. and decl. are listed in Columns 9 and 10 of Table 6. For motions with a significance of $>3 \sigma$, the total proper motion is listed in Column 11 along with the tangential velocity in Column 12 . 


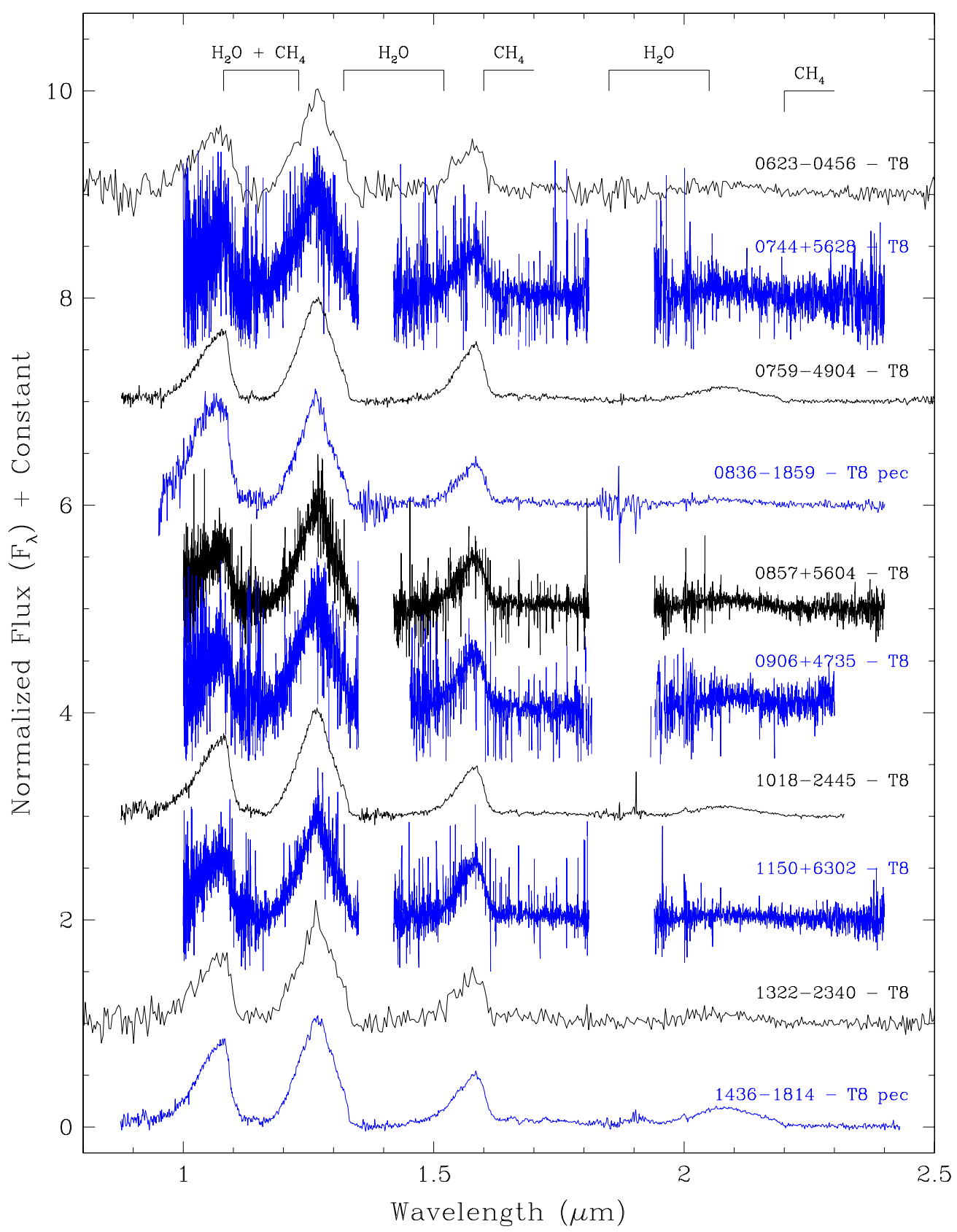

Figure 23. Near-infrared spectra of confirmed WISE brown dwarfs with spectral types of T8 (continued). Spectra have been normalized to one around $1.28 \mu \mathrm{m}$ and integral offsets have been added to the $y$-axis values to separate the spectra vertically. For some spectra, noisy data in the depths of the telluric water bands are not plotted. Prominent spectral features are marked.

(A color version of this figure is available in the online journal.)

\subsection{Space Density of Late-T Dwarfs}

The brown dwarf discoveries presented here represent only a fraction of the brown dwarf candidates identified so far from WISE data. WISE co-added data are not available across the entire sky, and many of those co-adds do not reach the full survey depth. Nonetheless, we can use these preliminary results to assess our progress toward completing the tally of cold brown dwarfs in the Solar Neighborhood and gauging the functional form of the mass function for these objects by using lower limits to their space densities.

Our goal is to complete an all-sky census of objects out to a specified maximum distance for each spectral subtype of T6 or later. Table 8 divides our discoveries into six spectral type bins (Column 1) from T6 through $>\mathrm{Y} 0$. The approximate range in effective temperature is given for each bin in Column 2. These temperature bins are assigned as follows. We took the values of $T_{\text {eff }}$ for T dwarfs of type T6 and later as computed by Warren et al. (2007), Delorme et al. (2008), Burningham et al. (2008), Burgasser et al. (2010b), Lucas et al. (2010), Burgasser et al. (2011a), Burningham et al. (2011b), Burningham et al. (2011a), Bochanski et al. (2011), and Cushing et al. (2011) or compiled by Kirkpatrick (2005). Then, when necessary, we reassigned spectral types to these objects so that they matched the near-infrared spectral classification scheme of Burgasser et al. (2006b) or its extension beyond T8 by Cushing et al. (2011). We then examined the distribution of temperature within each integral spectral type bin and found that a $150 \mathrm{~K}$ width for each 


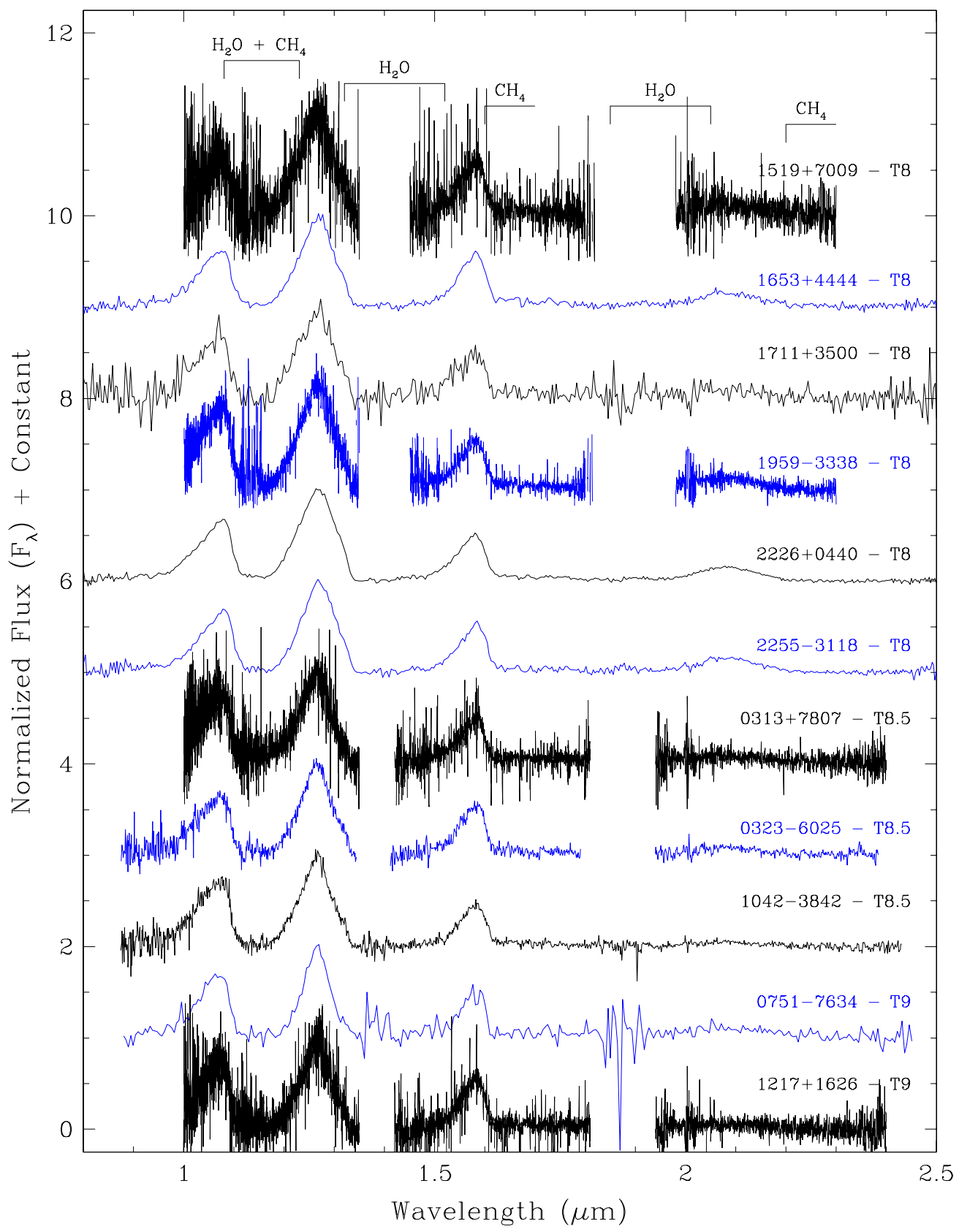

Figure 24. Near-infrared spectra of confirmed WISE brown dwarfs with spectral types from T8 (continued) to T9. (Additional T9 dwarfs are shown in Figure 25.) Spectra have been normalized to one around $1.28 \mu \mathrm{m}$ and integral offsets have been added to the $y$-axis values to separate the spectra vertically. For some spectra, noisy data in the depths of the telluric water bands are not plotted. Prominent spectral features are marked.

(A color version of this figure is available in the online journal.)

bin was enough to encompass the $T_{\text {eff }}$ values for most of the objects. The final assignments are given in Column 2 of Table 8.

As shown in Figure 30, the depth of our current search translates to different distances for each bin. In the spirit of determining the space density using a well defined census of the solar neighborhood, we limit our sample to those objects falling within $20 \mathrm{pc}$ of the Sun even if WISE can sample that spectral type to larger distances. Only in the last three bins- the T9-T9.5 bin and the Y dwarf bins-is WISE incomplete at this distance, so those bins are limited to volumes with smaller radii. These values, called $d_{\max }$, are listed in Column 3 .

Next, the number of objects per spectral type bin lying closer than the value of $d_{\max }$ is tabulated for previously published objects (Column 4), for our new WISE discoveries
(Column 5), and in total (Column 6). Distances are determined using trigonometric parallaxes, if available, or spectrophotometric estimates if no parallax has been measured. The resulting space density in each bin is given in Column 7.

This simple calculation of the space densities can be overestimated for the following reasons.

1. Spectrophotometric distance estimates have an inherent bias. The absolute magnitude versus spectral type relation is based on parallaxes, and those parallax measurements lead to a bias in estimated distances because a parallax value of $\pi_{\text {trig }} \pm \sigma$ is more likely to represent an object farther away $\left(\pi_{\text {trig }}-\sigma\right)$ than an object closer $\left(\pi_{\text {trig }}+\sigma\right)$ because the volume of space between parallax values of $\pi_{\text {trig }}$ and 


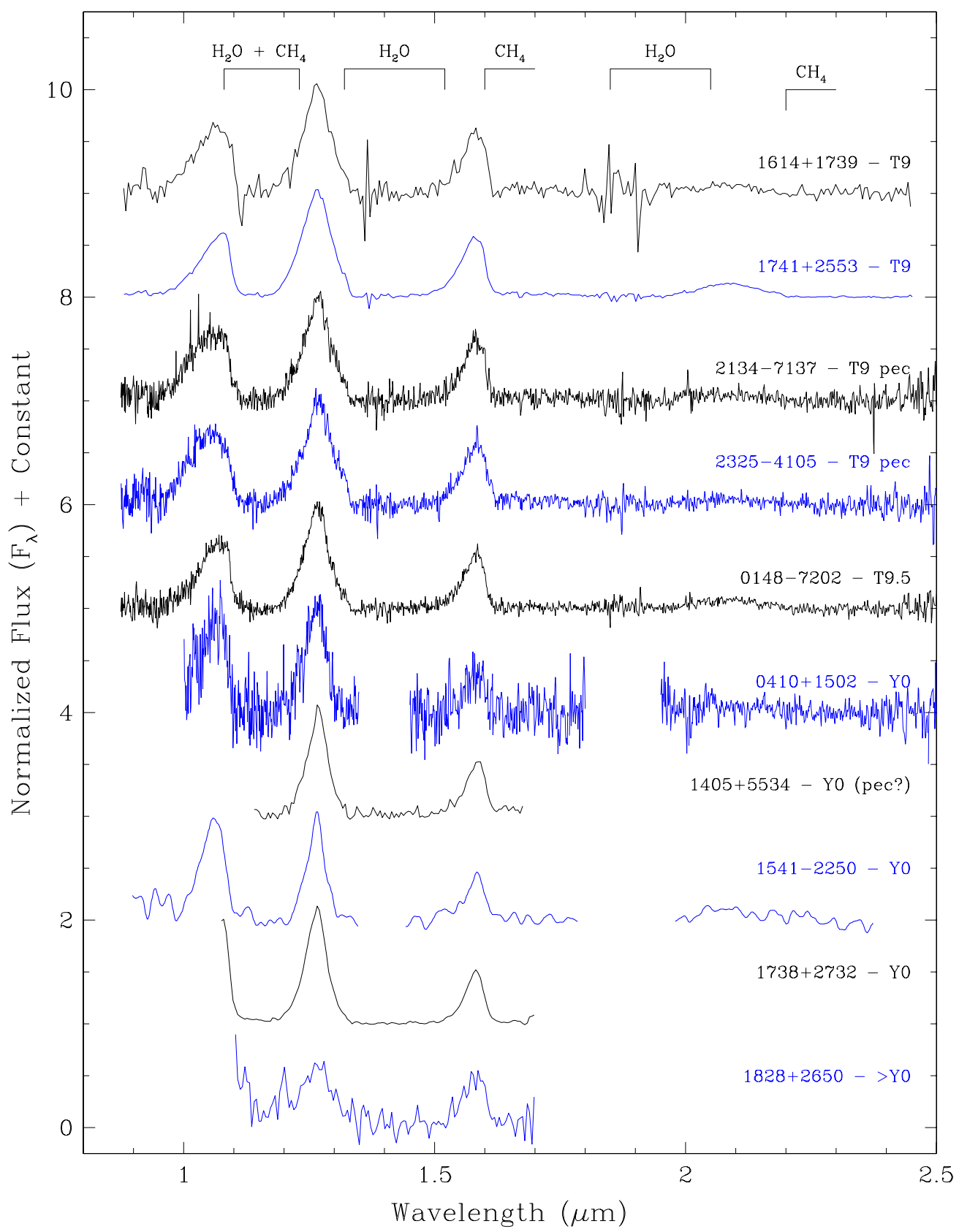

Figure 25. Near-infrared spectra of confirmed WISE brown dwarfs with spectral types from T9 (continued) to early-Y. Spectra have been normalized to one around $1.28 \mu \mathrm{m}$ and integral offsets have been added to the $y$-axis values to separate the spectra vertically. For some spectra, noisy data in the depths of the telluric water bands are not plotted. Prominent spectral features are marked.

(A color version of this figure is available in the online journal.)

$\pi_{\text {trig }}-\sigma$ is larger than that sampled between parallax values of $\pi_{\text {trig }}$ and $\pi_{\text {trig }}+\sigma$. Thus, the observed values of $\pi_{\text {trig }}$ are larger than the true values and the measured absolute values will be systematically too large. A correction can be applied that depends only on the value of $\sigma / \pi_{\text {trig }}$ (see Table 1 of Lutz \& Kelker 1973). Most of the parallaxes in Table 5 have $\sigma / \pi_{\text {trig }}$ values of less than $5 \%$ where the correction to the absolute magnitude is $\leqslant 0.02 \mathrm{mag}$, and those with larger errors have already been downweighted in our fit. We therefore conclude that the Lutz-Kelker effect is negligible here.

2. Both the Malmquist bias and the Eddington bias can be largely accounted for by limiting the sample over which we derive our space densities. Malmquist bias (Malmquist
1920), in which more luminous objects can preferentially bias statistics in a magnitude limited sample, can be eliminated by calculating space densities in narrow spectral type bins in which all objects have the same (or nearly the same) intrinsic luminosity. The Eddington bias (Eddington 1913; Eddington 1940), in which random errors will bias magnitude measures to brighter values due to the fact that there are more objects in the more distant (fainter) population than in the closer (brighter) one, can be reduced by operating at magnitudes where the random errors are still small. By using the brighter and better measured $W 2$ values to estimate distances, we can reduce the effects of Eddington bias on our derived densities. (For further discussion, see also Teerikorpi 2004.) 

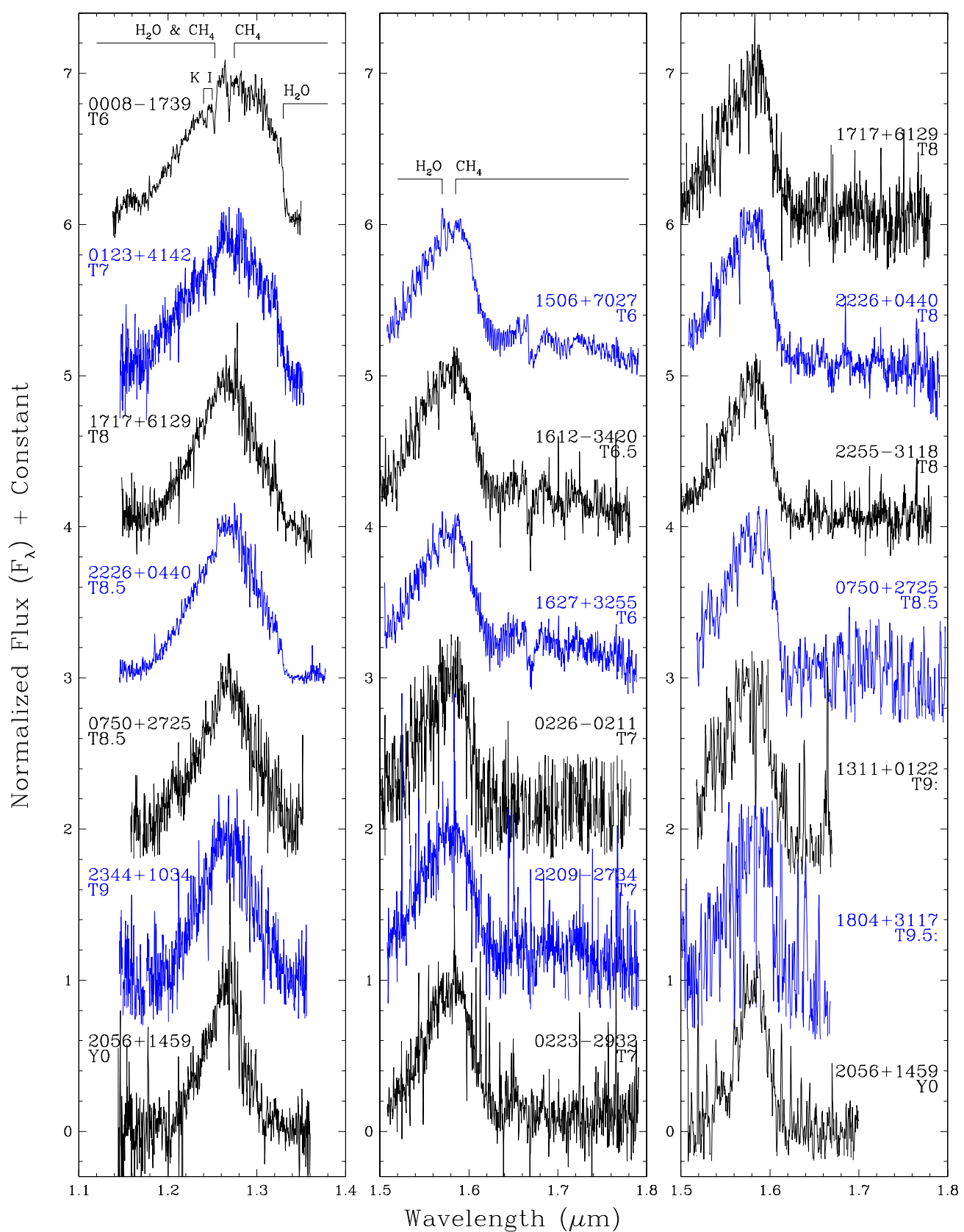

Figure 26. Keck/NIRSPEC spectra of confirmed WISE brown dwarfs. $J$-band spectra are shown in the left panel and $H$-band spectra are shown in the middle and right panels. Spectra have been normalized to one at peak flux and integral offsets have been added to the $y$-axis values to separate the spectra vertically. The bottom three spectra in the rightmost plot—-those of WISE 1311+0122, WISE 1804+3117, and WISE 2056+1459—-have been smoothed with a 5 pixel boxcar. Prominent spectral features are marked.

(A color version of this figure is available in the online journal.)

3. Unresolved binarity will cause distances to be underestimated. This may cause a more distant object to appear closer than it really is and falsely inflate the space density. Empirical data presented earlier can be used to estimate this degree of binary contamination. Figure 29 shows a well-defined binary sequence (red) overlying the sequence of single objects on the $M_{W 2}$ versus spectral type diagram. If we compute the ratio of known binaries to total objects between $\mathrm{L} 0$ and $\mathrm{T} 4$, we find that $12 / 35$, or $34 \%$, are binary. (See also Section 7.4 of Burgasser et al. 2006b for an in-depth discussion of intrinsic binarity, which varies from $\sim 20 \%$ at early-L to $\sim 42 \%$ at the $\mathrm{L} / \mathrm{T}$ transition.) While this is a sizable percentage, it does not mean that all of the unresolved binaries fall outside of the sample considered. Some small fraction will still be contained within the distance limit and will have been undercounted by a factor of two. Nonetheless, binarity is likely the largest contributor to inflating density estimates.

On the other hand, other biases discussed below lead to an underestimate in the space densities. These effects are believed to overwhelm the effects detailed above, and hence our simple space density calculations, although preliminary, can be considered as lower limits to the true densities.

1. Although WISE has taken data covering the entire sky at multiple epochs, the available co-added data cover less than 


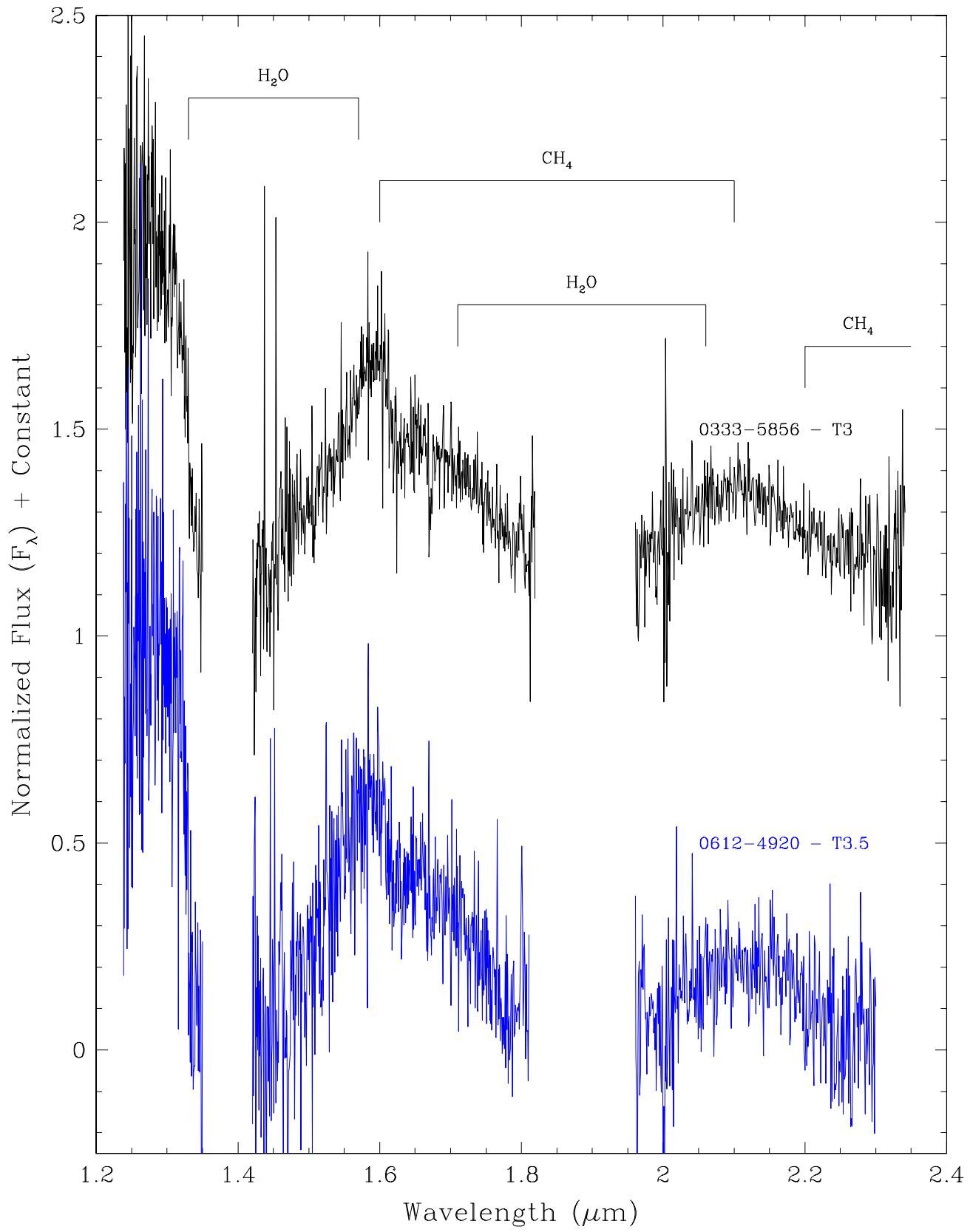

Figure 27. SOAR/OSIRIS spectra of two confirmed WISE brown dwarfs. Noisy data in the depths of the telluric water bands near 1.4 and $1.9 \mu \mathrm{m}$ are not plotted. Spectra have been normalized to one at peak flux and an offset of 1 has been added to the $y$-axis value of WISE 0333-5856 to separate it vertically from WISE 0612-4920. Prominent spectral features are marked.

(A color version of this figure is available in the online journal.)

$75 \%$ of the entire sky. Also, none of the sky has been coadded to its full depth using all available frames and the detection threshold for first-pass processing was set higher, in units of $\mathrm{S} / \mathrm{N}$, than it will be for final processing. The latter points are particularly important as they will, in the future, enable more robust colors or color limits for potential $\mathrm{Y}$ dwarf candidates. Moreover, we have followed up less than $50 \%$ of the brown dwarf candidates already culled from sections of the sky for which we have access. Thus, we believe that our current space density estimates are gross underestimates.

2. Except for 2MASS, other surveys providing data in Column 4 of Table 8 do not have all-sky coverage and can only provide limited help in completing this nearby sample.
Moreover, none of the current or planned ground-based surveys canvassing the sky for brown dwarfs can reach sizable populations of the coldest objects because Y dwarfs are intrinsically dim at ground-observable wavelengths. This is highlighted in Figure 31, which shows the absolute $H$-band magnitude as a function of spectral type. Note that the Y0 dwarf WISE $1541-2250$ has $M_{H}=23.7 \pm 0.9 \mathrm{mag}$; its absolute magnitude is $M_{J}=23.9 \pm 0.8 \mathrm{mag}$ and it is presumably even fainter than this shortward of $J$ band. WISE operates at wavelengths where these objects are their brightest-five thousand times brighter at $W 2$ than at the $J$ and $H$ bands, in the case of WISE $1828+2650-$ so it is the only survey capable of detecting the coldest brown dwarfs in significant numbers. 


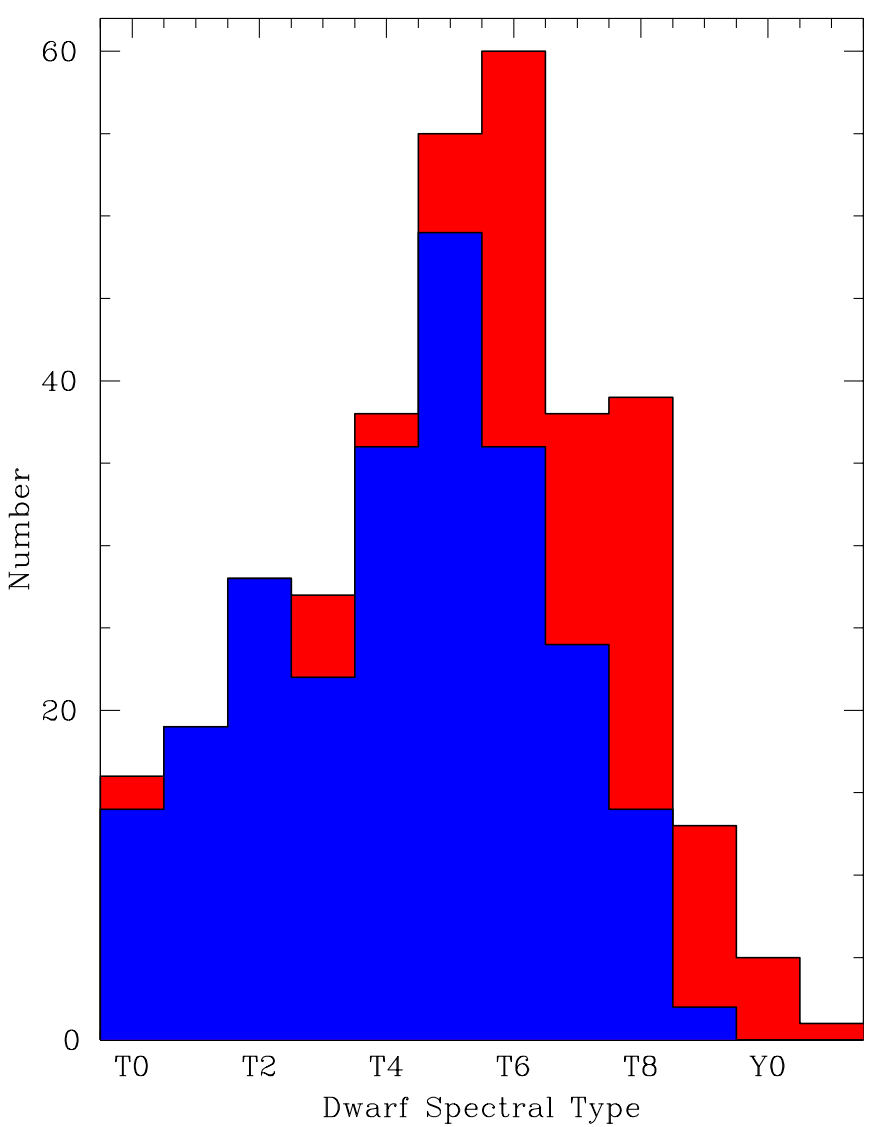

Figure 28. Histogram of spectral types for published objects with near-infrared types of T0 or later. The distribution of types of previously published brown dwarfs is shown in blue and the distribution of new types for discoveries in this paper is shown in red. Objects are counted into bins of integral subtypes (e.g., objects of type T7 and T7.5 are shown in the T7 bin). The previously published objects were taken from DwarfArchives.org on 2011 May 15 and are supplemented with new discoveries by Albert et al. (2011) and Burningham et al. (2011b).

(A color version of this figure is available in the online journal.)

3. As mentioned earlier, the $M_{W 2}$ versus spectral type relation of Figure 29 likely overpredicts distances to dwarfs of type $\geqslant$ T9. This means that our surveyed volume may be overestimated, leading to an underestimate of the space density.

4. Despite the all-sky coverage of WISE, the galactic plane will restrict our ability to probe to the same depths as other parts of the sky due to higher backgrounds and confusion. This loss of coverage is not currently accounted for in our density estimates.

We have checked the distance distribution of objects in each spectral type bin by performing the $V / V_{\max }$ test (Column 8 of Table 8). This test was first proposed by Schmidt (1968) to check the uniformity of a distribution of objects in space. The quantity $V$ is the volume of space interior to object $i$ at distance $d_{i}$, and $V_{\max }$ is the full volume of space contained within the distance limit, $d_{\max }$, of the sample. For a uniform sample, the average value, $\left\langle V / V_{\max }\right\rangle$, should be 0.5 because half of the sample should lie in the nearer half of the volume and the rest should lie in the farther half. If this number is not near 0.5 , then the sample is either non-isotropic or incomplete. For our sample we find that the $\mathrm{T}$ dwarf bins have $\left\langle V / V_{\max }\right\rangle$ values considerably less than 0.5. This points to incompleteness in the sample-our low $\left\langle V / V_{\max }\right\rangle$ values are almost certainly a consequence of the

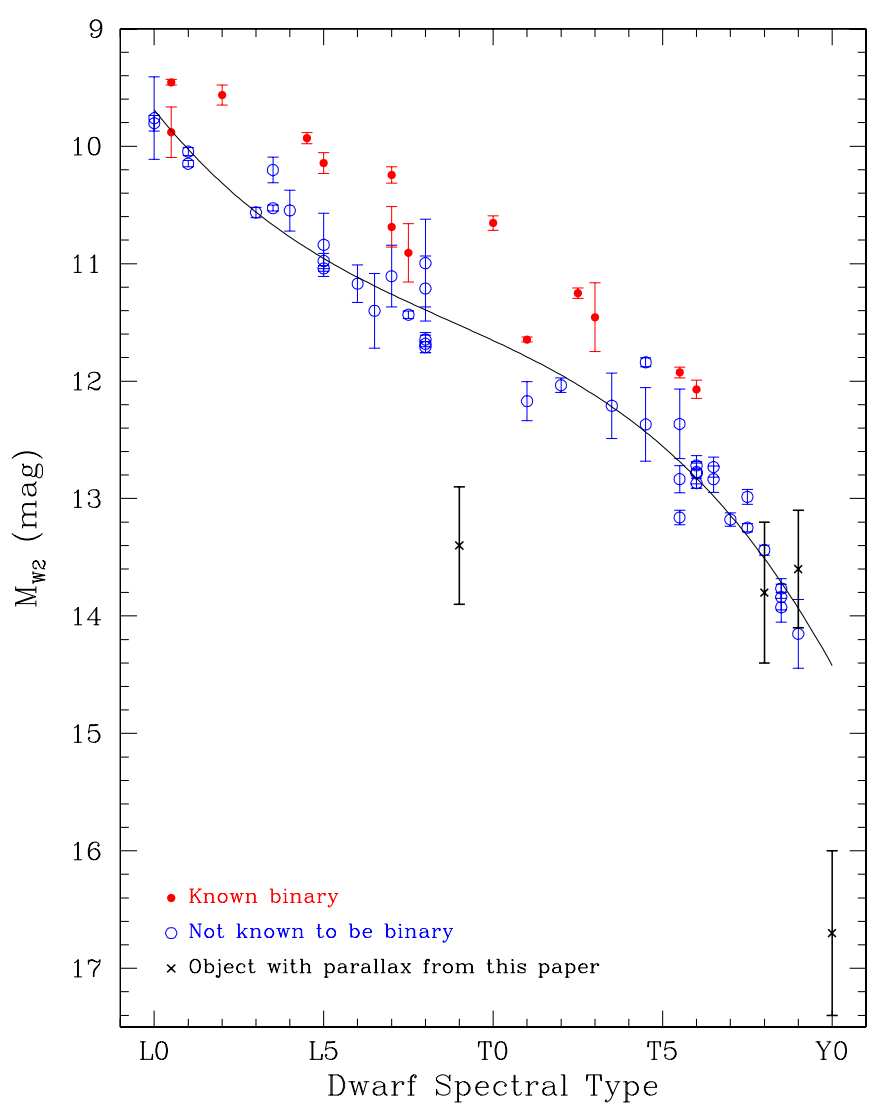

Figure 29. Absolute $W 2$ magnitude plotted against spectral type for objects with measured trigonometric parallaxes. Red points are those objects known to be binary through high-resolution imaging. All others are shown as blue points. The solid line shows a third-order relation fit through the blue points, as described in the text. WISE discoveries with trigonometric parallaxes from Table 7 are shown with black x's. The point for WISE 1541-2250 at lower right, if confirmed via continued astrometric monitoring, suggests that an extrapolation of the fitted relation cooler than $\mathrm{T} 9$ may result in $\mathrm{Y}$ dwarf spectrophotometric distance estimates that are too large.

(A color version of this figure is available in the online journal.)

fact that the brighter (closer) candidates tend to be followed up first. This gives further credence to the assertion that our number densities are lower limits only. For the Y dwarf bins, however, the values of $\left\langle V / V_{\max }\right\rangle$ are above 0.5 , which further suggests that our assumed distances to these objects are overestimates.

Figure 32 shows these preliminary results (Column 9 of Table 8) relative to measurements made by other surveys and relative to predictions based on different forms of the underlying mass function. Previous results by Metchev et al. (2008), Burningham et al. (2010b), and Reylé et al. (2010) are shown by the open symbols and, with the possible exception of the Burningham et al. (2010b) point, support values of $\alpha$ of zero or greater, where the functional form is given as $d N / d M \propto M^{-\alpha}$. Our incomplete, volume-limited sample so far fails to put tighter constraints on the mass function at warmer temperatures than previously published work, but the preliminary lower limit to the $\mathrm{Y} 0$ space density already rules out the $\alpha=-1.0$ model and may soon, with additional $\mathrm{Y} 0$ discoveries, be able to distinguish between the $\alpha=0.0$ and $\alpha=+1.0$ models. Results for the early-Y dwarfs already suggest that the low-mass cutoff of star formation must be below $10 M_{\text {Jup }}$ if $\alpha \leqslant 0$. This result is in accordance with findings in young star formation regions (e.g., Luhman et al. 2000; Muench et al. 2002; Lucas et al. 2006; Luhman 2007), but the derived masses for those objects 
Table 5

L and T Dwarfs with Measured Trigonometric Parallaxes

\begin{tabular}{|c|c|c|c|c|c|}
\hline Object Name & $\begin{array}{c}\pi_{\text {trig }} \\
(\text { mas) } \\
(2)\end{array}$ & $\begin{array}{l}\text { Ref. } \\
\text { (3) }\end{array}$ & $\begin{array}{c}\text { NIR Sp. } \\
\text { Type } \\
(4)\end{array}$ & $\begin{array}{c}W 2 \\
(\mathrm{mag}) \\
(5)\end{array}$ & $\begin{array}{c}H \\
(\mathrm{mag}) \\
(6)\end{array}$ \\
\hline \multicolumn{6}{|l|}{ (a) Objects not known to be binary } \\
\hline UGPS J072227.51-054031.2 & $246 \pm 33$ & 2 & T9 & $12.197 \pm 0.027$ & $16.147 \pm 0.205$ \\
\hline DENIS J081730.0-615520 & $203 \pm 13$ & 3 & T6 & $11.237 \pm 0.017$ & $13.526 \pm 0.031$ \\
\hline DENIS-P J0255-4700 & $201.37 \pm 3.89$ & 4 & L8 & $10.190 \pm 0.021$ & $12.204 \pm 0.024$ \\
\hline 2MASSI J0415195-093506 & $174.34 \pm 2.76$ & 5 & $\mathrm{~T} 8$ & $12.232 \pm 0.026$ & $15.537 \pm 0.113$ \\
\hline Gliese 570D & $169.3 \pm 1.7$ & 6 & $\mathrm{~T} 7.5$ & $12.105 \pm 0.026$ & $15.268 \pm 0.089$ \\
\hline 2MASSI J0937347+293142 & $163.39 \pm 1.76$ & 7 & T6 & $11.652 \pm 0.023$ & $14.703 \pm 0.068$ \\
\hline 2MASSW J1507476-162738 & $136.4 \pm 0.6$ & 8 & L5 & $10.367 \pm 0.022$ & $11.895 \pm 0.024$ \\
\hline 2MASSW J0036159+182110 & $114.2 \pm 0.8$ & 8 & $\mathrm{~L} 3.5$ & $10.239 \pm 0.020$ & $11.588 \pm 0.029$ \\
\hline 2MASSI J0727182+171001 & $110.14 \pm 2.34$ & 5 & $\mathrm{~T} 7$ & $12.969 \pm 0.033$ & $15.756 \pm 0.171$ \\
\hline CFBDS J005910.90-011401.3 & $108.2 \pm 5.0$ & 1 & $\mathrm{~T} 8.5$ & $13.668 \pm 0.044$ & $18.270 \pm 0.050$ \\
\hline 2MASS J05591914-1404488 & $97.7 \pm 1.3$ & 8 & $\mathrm{~T} 4.5$ & $11.891 \pm 0.023$ & $13.679 \pm 0.044$ \\
\hline ULAS J133553.45+113005.2 & $96.7 \pm 3.2$ & 1 & $\mathrm{~T} 8.5$ & $13.839 \pm 0.046$ & $18.250 \pm 0.010$ \\
\hline 2MASS J12373919+6526148 & $96.07 \pm 4.78$ & 5 & T6.5 & $12.922 \pm 0.028$ & $15.739 \pm 0.145$ \\
\hline 2MASSI J0825196+211552 & $93.8 \pm 1.0$ & 8 & L7.5 & $11.574 \pm 0.022$ & $13.792 \pm 0.032$ \\
\hline 2MASSI J0243137-245329 & $93.62 \pm 3.63$ & 5 & T6 & $12.929 \pm 0.030$ & $15.137 \pm 0.109$ \\
\hline SDSSp J162414.37+002915.6 & $90.9 \pm 1.2$ & 9 & T6 & $13.077 \pm 0.032$ & $15.524 \pm 0.100$ \\
\hline 2MASSI J1217110-031113 & $90.8 \pm 2.2$ & 9 & $\mathrm{~T} 7.5$ & $13.195 \pm 0.035$ & $15.748 \pm 0.119$ \\
\hline 2MASSI J1546291-332511 & $88.0 \pm 1.9$ & 9 & T5.5 & $13.439 \pm 0.039$ & $15.446 \pm 0.092$ \\
\hline SDSSp J125453.90012247.4 & $84.9 \pm 1.9$ & 8 & $\mathrm{~T} 2$ & $12.391 \pm 0.037$ & $14.090 \pm 0.025$ \\
\hline ULAS J003402.77-005206.7 & $78.0 \pm 3.6$ & 1 & $\mathrm{~T} 8.5$ & $14.465 \pm 0.076$ & $18.490 \pm 0.040$ \\
\hline SDSSp J053951.99-005902.0 & $76.12 \pm 2.17$ & 5 & L5 & $11.569 \pm 0.021$ & $13.104 \pm 0.026$ \\
\hline 2MASSW J1439284+192915 & $69.6 \pm 0.5$ & 8 & $\mathrm{~L} 1$ & $10.936 \pm 0.021$ & $12.041 \pm 0.019$ \\
\hline 2MASSI J2356547-155310 & $68.97 \pm 3.42$ & 5 & $\mathrm{~T} 5.5$ & $13.641 \pm 0.041$ & $15.630 \pm 0.100$ \\
\hline SDSSp J134646.45-003150.4 & $68.3 \pm 2.3$ & 9 & $\mathrm{~T} 6.5$ & $13.560 \pm 0.042$ & $15.459 \pm 0.118$ \\
\hline 2MASSW J1632291+190441 & $65.6 \pm 2.1$ & 8 & L8 & $12.598 \pm 0.028$ & $14.612 \pm 0.038$ \\
\hline DENIS-P J1058.7-1548 & $57.7 \pm 1.0$ & 8 & L3 & $11.758 \pm 0.023$ & $13.226 \pm 0.025$ \\
\hline 2MASSW J1658037+702701 & $53.9 \pm 0.7$ & 8 & $\mathrm{~L} 1$ & $11.388 \pm 0.022$ & $12.470 \pm 0.032$ \\
\hline Gliese 584C & $53.70 \pm 1.24$ & 6 & L8 & $12.996 \pm 0.033$ & $14.928 \pm 0.081$ \\
\hline SDSSp J132629.82-003831.5 & $49.98 \pm 6.33$ & 5 & L8 & $12.718 \pm 0.030$ & $15.050 \pm 0.060$ \\
\hline SDSS J015141.69+124429.6 & $46.73 \pm 3.37$ & 5 & $\mathrm{~T} 1$ & $13.823 \pm 0.053$ & $15.603 \pm 0.112$ \\
\hline SDSSp J144600.60+002452.0 & $45.46 \pm 3.25$ & 5 & L6 & $12.882 \pm 0.034$ & $14.514 \pm 0.035$ \\
\hline SDSSp J175032.96+175903.9 & $36.24 \pm 4.53$ & 5 & $\mathrm{~T} 3.5$ & $14.414 \pm 0.057$ & $15.952 \pm 0.132$ \\
\hline 2MASSW J0030300-145033 & $37.42 \pm 4.50$ & 5 & L7 & $13.241 \pm 0.034$ & $15.273 \pm 0.100$ \\
\hline 2MASSI J1711457+223204 & $33.11 \pm 4.81$ & 5 & L6.5 & $13.802 \pm 0.040$ & $15.797 \pm 0.109$ \\
\hline GD $165 \mathrm{~B}^{\mathrm{a}}$ & $31.7 \pm 2.5$ & 10 & $\mathrm{~L} 4$ & $13.042 \pm 0.032$ & $14.781 \pm 0.070$ \\
\hline 2MASSW J1328550+211449 & $31.0 \pm 3.8$ & 8 & L5 & $13.383 \pm 0.036$ & $15.002 \pm 0.081$ \\
\hline 2MASSW J0326137+295015 & $31.0 \pm 1.5$ & 8 & L3.5 & $12.746 \pm 0.029$ & $14.395 \pm 0.050$ \\
\hline SDSSp J003259.36+141036.6 & $30.14 \pm 5.16$ & 5 & L8 & $13.600 \pm 0.041$ & $15.648 \pm 0.142$ \\
\hline SDSS J020742.48+000056.2 & $29.3 \pm 4.0$ & 1 & $\mathrm{~T} 4.5$ & $15.035 \pm 0.100$ & $>16.396$ \\
\hline ULAS J082707.67-020408.2 & $26.0 \pm 3.1$ & 1 & T5.5 & $15.290 \pm 0.142$ & $17.440 \pm 0.050$ \\
\hline HD 89744B & $25.65 \pm 0.70$ & 6 & L0 & $12.759 \pm 0.029$ & $14.022 \pm 0.033$ \\
\hline SDSSp J225529.09-003433.4 & $16.19 \pm 2.59$ & 5 & L0 & $13.715 \pm 0.052$ & $14.756 \pm 0.058$ \\
\hline \multicolumn{6}{|l|}{ (b) Known binaries/multiples: } \\
\hline$\epsilon$ Ind $\mathrm{Bab}$ & $275.76 \pm 0.69$ & 6 & $\mathrm{~T} 1$ & $9.443 \pm 0.020$ & $11.510 \pm 0.020$ \\
\hline 2MASSI J0746425+200032 & $81.9 \pm 0.3$ & 8 & L0.5 & $9.889 \pm 0.022$ & $11.007 \pm 0.022$ \\
\hline 2MASS J12255432-2739466 & $75.1 \pm 2.5$ & 9 & $\mathrm{~T} 6$ & $12.692 \pm 0.030$ & $15.098 \pm 0.081$ \\
\hline 2MASSI J1534498-295227 & $73.6 \pm 1.2$ & 9 & $\mathrm{~T} 5.5$ & $12.592 \pm 0.029$ & $14.866 \pm 0.102$ \\
\hline SDSSp J042348.57-041403.5 & $65.93 \pm 1.70$ & 5 & T0 & $11.559 \pm 0.025$ & $13.463 \pm 0.035$ \\
\hline HN Peg B & $54.37 \pm 0.85$ & 6 & $\mathrm{~T} 2.5$ & $12.574 \pm 0.029$ & $15.400 \pm 0.030$ \\
\hline Kelu-1AB & $53.6 \pm 2.0$ & 8 & $\mathrm{~L} 2$ & $10.918 \pm 0.025$ & $12.392 \pm 0.025$ \\
\hline DENIS-P J0205.4-1159 & $50.6 \pm 1.5$ & 8 & L7 & $11.724 \pm 0.030$ & $13.568 \pm 0.037$ \\
\hline DENIS-P J1228.2-1547 & $49.4 \pm 1.9$ & 8 & L5 & $11.675 \pm 0.034$ & $13.347 \pm 0.032$ \\
\hline Gliese 417BC & $46.04 \pm 0.90$ & 6 & L4.5 & $11.616 \pm 0.023$ & $13.499 \pm 0.032$ \\
\hline 2MASSW J1728114+394859 & $41.49 \pm 3.26$ & 5 & L7 & $12.597 \pm 0.017$ & $14.756 \pm 0.066$ \\
\hline G 124-62B & $36.39 \pm 3.57$ & 4 & L0.5 & $12.077 \pm 0.026$ & $13.190 \pm 0.031$ \\
\hline SDSS J102109.69-030420.1 & $34.4 \pm 4.6$ & 9 & $\mathrm{~T} 3$ & $13.773 \pm 0.041$ & $15.346 \pm 0.101$ \\
\hline 2MASSW J2101154+175658 & $30.14 \pm 3.42$ & 5 & L7.5 & $13.512 \pm 0.036$ & $15.861 \pm 0.182$ \\
\hline
\end{tabular}

Notes. ${ }^{\text {a }}$ It is assumed that most of the $W 2$ flux comes from the $\mathrm{L}$ dwarf companion and not the white dwarf primary.

References. (1) Marocco et al. 2010; (2) Lucas et al. 2010; (3) Artigau et al. 2010; (4) Costa et al. 2006; (5) Vrba et al. 2004; (6) Perryman et al. 1997; (7) Schilbach et al. 2009; (8) Dahn et al. 2002; (9) Tinney et al. 2003; (10) van Altena et al. 1995. 
Table 6

Astrometry for WISE Brown Dwarf Discoveries

\begin{tabular}{|c|c|c|c|c|c|c|c|c|c|c|c|}
\hline $\begin{array}{l}\text { Object Name } \\
\text { (1) }\end{array}$ & $\begin{array}{l}\text { Dist. Est. } \\
\text { (pc) } \\
\text { (2) }\end{array}$ & $\begin{array}{l}\text { R.A. (J2000) } \\
\text { (deg) } \\
\text { (3) }\end{array}$ & $\begin{array}{c}\text { Decl. (J2000) } \\
\text { (deg) } \\
(4)\end{array}$ & $\begin{array}{l}\text { R.A. Err } \\
(\operatorname{arcsec}) \\
(5)\end{array}$ & $\begin{array}{l}\text { Decl. Err } \\
(\operatorname{arcsec}) \\
(6)\end{array}$ & $\begin{array}{c}\text { Reference } \\
\text { (7) }\end{array}$ & $\begin{array}{l}\text { MJD } \\
(8)\end{array}$ & $\begin{array}{c}\mu_{\alpha} \\
\left(\operatorname{arcsec} \mathrm{yr}^{-1}\right) \\
(9)\end{array}$ & $\begin{array}{c}\mu_{\delta} \\
\left(\operatorname{arcsec} \mathrm{yr}^{-1}\right) \\
(10)\end{array}$ & $\begin{array}{c}\mu_{\text {total }} \\
\left(\operatorname{arcsec} \mathrm{yr}^{-1}\right) \\
(11)\end{array}$ & $\begin{array}{c}v_{\tan } \\
\left(\mathrm{km} \mathrm{s}^{-1}\right) \\
(12)\end{array}$ \\
\hline \multirow[t]{3}{*}{ WISE 0008-1739 } & 22.0 & 2.2073557 & -17.6563363 & 0.179 & 0.185 & WISE epoch 1 & 55363.39 & $-0.116 \pm 0.589$ & $-1.108 \pm 0.532$ & & \\
\hline & & 2.2073061 & -17.6565639 & 0.279 & 0.293 & WISE epoch 2 & 55542.36 & & & & \\
\hline & & 2.2073379 & -17.6564659 & 0.485 & 0.309 & Spitzer & 55571 & & & & \\
\hline \multirow[t]{3}{*}{ WISE $0031-3840$} & 22.0 & 7.830238 & -38.676575 & 0.06 & 0.07 & 2MASS PSC & 51391.3054 & $0.548 \pm 0.006$ & $-0.049 \pm 0.007$ & $0.550 \pm 0.009$ & $57.4 \pm 1.0$ \\
\hline & & 7.8323524 & -38.6766992 & 0.045 & 0.044 & WISE epoch 1 & 55357.83 & & & & \\
\hline & & 7.8324281 & -38.6767498 & 0.046 & 0.044 & WISE epoch 2 & 55535.75 & & & & \\
\hline \multirow[t]{4}{*}{ WISE 0049+0441 } & 19.2 & 12.367782 & 4.682658 & 0.07 & 0.12 & 2MASS PSC & 51768.4283 & $0.315 \pm 0.008$ & $0.228 \pm 0.012$ & $0.389 \pm 0.014$ & $35.4 \pm 1.3$ \\
\hline & & 12.3686648 & 4.6833142 & 0.053 & 0.053 & WISE epoch 1 & 55382.38 & & & & \\
\hline & & 12.3686625 & 4.6832837 & 0.066 & 0.062 & WISE epoch 2 & 55560.23 & & & & \\
\hline & & 12.3686292 & 4.6833140 & 0.206 & 0.260 & Spitzer & 55591 & & & & \\
\hline
\end{tabular}

Notes.

${ }^{a}$ Gelino et al. (2011) find this object to be a binary. Their revised distance to this system is $12.3 \pm 2.3 \mathrm{pc}$.

${ }^{\mathrm{b}}$ Gelino et al. (2011) find this object to be a binary. Their revised distance to this system is $40.1 \pm 3.0 \mathrm{pc}$.

(This table is available in its entirety in a machine-readable form in the online journal. A portion is shown here for guidance regarding its form and content.) 
Table 7

Preliminary Parallaxes and Absolute Magnitudes for WISE Brown Dwarf Discoveries

\begin{tabular}{|c|c|c|c|c|c|c|}
\hline $\begin{array}{l}\text { Object } \\
\text { Name } \\
(1)\end{array}$ & $\begin{array}{c}\text { Near-infrared } \\
\text { Spectral Type } \\
\text { (2) }\end{array}$ & $\begin{array}{c}\pi_{\text {trig }} \\
(\operatorname{arcsec}) \\
(3)\end{array}$ & $\begin{array}{l}\text { Dist. Range }( \pm 1 \sigma) \\
(\mathrm{pc}) \\
(4)\end{array}$ & $\begin{array}{c}M_{J}^{\mathrm{a}} \\
(\mathrm{mag}) \\
(5)\end{array}$ & $\begin{array}{c}M_{H}^{\mathrm{a}} \\
(\mathrm{mag}) \\
(6)\end{array}$ & $\begin{array}{c}M_{W 2} \\
(\mathrm{mag}) \\
(7)\end{array}$ \\
\hline WISE $0254+0223$ & T8 & $0.165 \pm 0.046$ & $4.7-8.4$ & $17.0 \pm 0.6$ & $17.4 \pm 0.6$ & $13.8 \pm 0.6$ \\
\hline WISE $1541-2250$ & Y0 & $0.351 \pm 0.108$ & $2.2-4.1$ & $23.9 \pm 0.8$ & $23.7 \pm 0.9$ & $16.7 \pm 0.7$ \\
\hline WISE 1647+5632 & L9 pec (red) & $0.116 \pm 0.029$ & $6.9-11.5$ & $16.9 \pm 0.5$ & $15.7 \pm 0.5$ & $13.4 \pm 0.5$ \\
\hline WISE $1741+2553$ & T9 & $0.182 \pm 0.038$ & $4.5-6.9$ & $17.8 \pm 0.5$ & $17.9 \pm 0.5$ & $13.6 \pm 0.5$ \\
\hline
\end{tabular}

Notes. ${ }^{a}$ Absolute $J$ and $H$ magnitudes for WISE $0254+0223$ and WISE $1541-2250$ are on the MKO filter system; magnitudes for WISE $1647+5632$ and WISE $1741+2553$ are on the 2MASS filter system.

Table 8

Preliminary Space Densities for an All-sky, Volume-limited Sample of Late-T and Y Dwarfs

\begin{tabular}{lcccccccc}
\hline \hline $\begin{array}{l}\text { Spectral Type } \\
\text { Range } \\
(1)\end{array}$ & $\begin{array}{c}\text { Approx. } T_{\text {eff }} \\
\text { Range }(\mathrm{K})\end{array}$ & $\begin{array}{c}d_{\max }(\mathrm{pc}) \\
(2)\end{array}$ & $\begin{array}{c}\text { No. from Previous } \\
\text { Surveys }\end{array}$ & $\begin{array}{c}\text { No. from WISE } \\
\text { to Date } \\
(3)\end{array}$ & $\begin{array}{c}\text { Total No. } \\
\text { to Date } \\
(6)\end{array}$ & $\begin{array}{c}\text { Obs. Space Density } \\
\left(\text { No. pc }^{-3}\right) \\
(7)\end{array}$ & $\begin{array}{c}\left\langle V / V_{\max }\right\rangle \\
(8)\end{array}$ & $\begin{array}{c}\text { No. per 100 K Bin } \\
\mathrm{w} / \mathrm{in} 10 \mathrm{pc} \\
(9)\end{array}$ \\
\hline T6-T6.5 & $900-1050$ & 20 & 14 & 16 & 30 & $>9.0 \mathrm{e}-4$ & 0.36 & $>2.5$ \\
T7-T7.5 & $750-900$ & 20 & 13 & 12 & 25 & $>7.5 \mathrm{e}-4$ & 0.34 & $>2.1$ \\
T8-T8.5 & $600-750$ & 20 & 9 & 26 & 35 & $>1.0 \mathrm{e}-3$ & 0.30 & $>2.9$ \\
T9-T9.5 & $450-600$ & 15 & 1 & 9 & 10 & $>7.1 \mathrm{e}-4$ & 0.40 & $>2.0$ \\
Y0 & $300-450$ & 10 & 0 & 4 & 4 & $>9.5 \mathrm{e}-4$ & 0.59 & $>2.7$ \\
>Y0 & $<300$ & 10 & 0 & 1 & 1 & $>2.4 \mathrm{e}-4$ & 0.83 & $>0.7$ \\
\hline
\end{tabular}

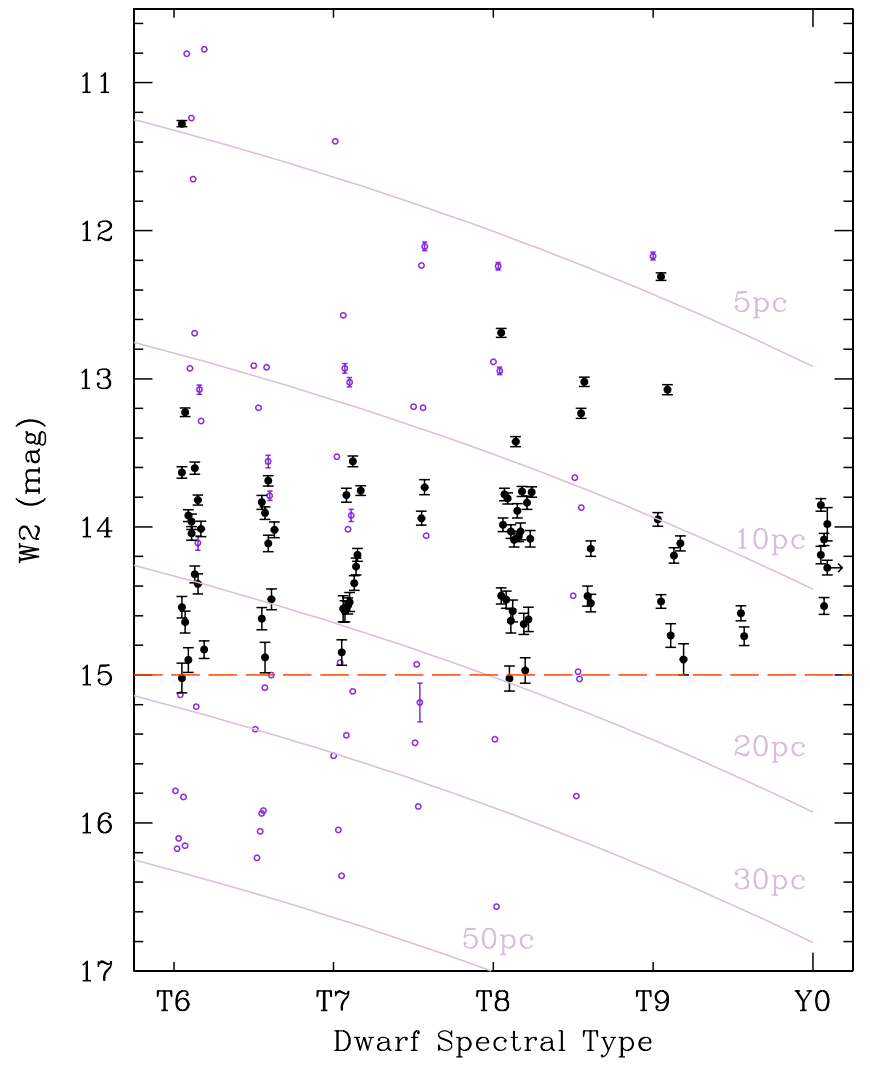

Figure 30. $W 2$ vs. spectral type for previously known objects (open, blue violet points) and WISE discoveries (solid, black points). Slight offsets have been added to the spectral subclass of each object so that points suffer from less overlap along the $x$-axis. The distance relation from Figure 29 is plotted at various distances from 5 to $50 \mathrm{pc}$ (gray lines) to aid the viewer in estimating distances to plotted objects. The dashed line in orange red shows the approximate W2 magnitude limit of our current WISE search. (Note. Error bars are shown on the open points when $W 2$ photometry is known. Otherwise, open points are plotted at the $W 2$ magnitudes estimated from the objects' spectral types and near-infrared magnitudes and are plotted without error bars.)

(A color version of this figure is available in the online journal.)

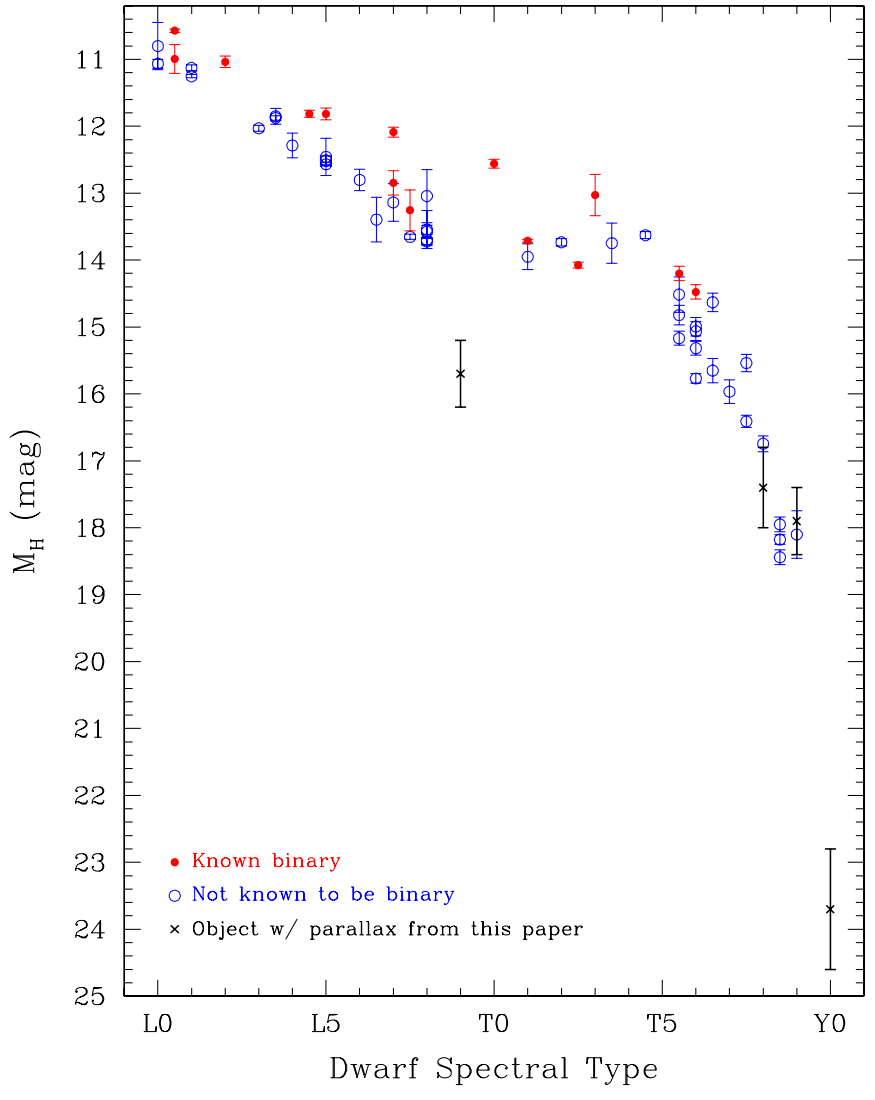

Figure 31. Absolute $H$ magnitude plotted against spectral type for objects with measured trigonometric parallaxes in Figure 29. Note the rapid dimming of the $H$-band magnitude at the latest $\mathrm{T}$ types. The point for WISE 1541-2250 at lower right, if confirmed via continued astrometric monitoring, suggests that this $H$-band dimming accelerates as objects cool to the Y dwarf class.

(A color version of this figure is available in the online journal.) 


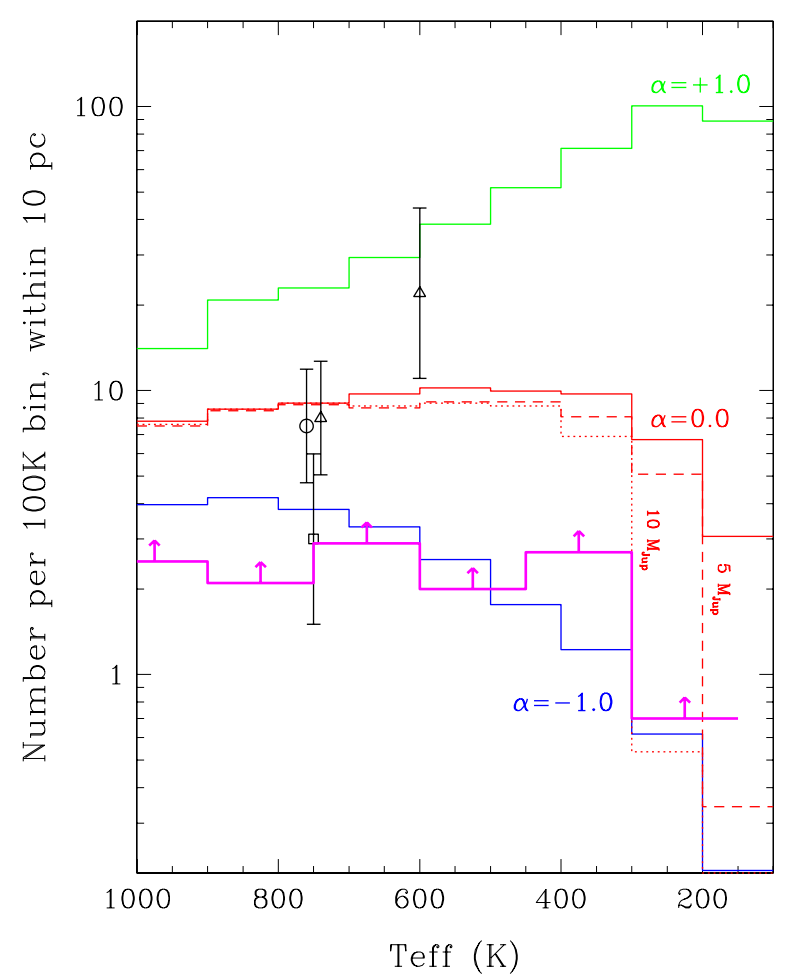

Figure 32. Predicted number of brown dwarfs within $10 \mathrm{pc}$ for three different mass functions $\left(d N / d M \propto M^{-\alpha}\right.$ with $\alpha=-1,0,1$ shown in green, red, and blue, respectively) having a minimum formation mass of $1 M_{\text {Jup }}$ (Burgasser 2004). Also shown for the $\alpha=0$ model (dashed and dotted red lines) is the change in the expected number of brown dwarfs when the minimum formation mass is varied. Recent measurements of the observed space densities of T dwarfs are shown as open symbols-Metchev et al. (2008) (circle), Burningham et al. (2010b) (square), and Reylé et al. (2010) (triangles). Lower limits to the space densities using a full accounting of objects in the Solar Neighborhood and based largely on early WISE results (Table 8) is shown in magenta.

(A color version of this figure is available in the online journal.)

should be considered cautiously, as discussed in Baraffe et al. (2003), because models with ages of $\sim 1$ Myr or younger are highly sensitive to untested assumptions about initial conditions. Using models of older ages is far less sensitive to these assumptions, so field brown dwarfs provide a better check of star formation's low-mass cutoff. Model fits by Cushing et al. (2011) suggest that our Y dwarf discoveries have masses as high as $30 M_{\text {Jup }}$ or as low as $3 M_{\text {Jup }}$ or less, which agrees roughly with the mass values inferred from Figure 32.

\section{CONCLUSIONS}

This paper represents the culmination of a year's worth of effort following up the first batch of brown dwarf candidates identified by WISE. There are many hundreds more candidates still being scrutinized, and there are still areas of sky not yet searched. It is therefore clear that these first hundred brown dwarf discoveries are harbingers of a much larger trove of brown dwarfs yet to be uncovered by WISE. Not only is the WISE data archive uniquely suited to finding even colder objects than the current batch of early-Y dwarfs, the all-sky and multiepoch nature of the mission will enable many other brown dwarf studies - the search for the lowest mass objects in nearby moving groups, hunting for low-metallicity objects via their high proper motions, etc.- that are well beyond the scope of the photometric search presented here.
This publication makes use of data products from the Widefield Infrared Survey Explorer, which is a joint project of the University of California, Los Angeles, and the Jet Propulsion Laboratory/California Institute of Technology, funded by the National Aeronautics and Space Administration. We acknowledge fruitful discussions with Tim Conrow, Roc Cutri, and Frank Masci, and acknowledge assistance with Magellan/FIRE observations by Emily Bowsher. This publication also makes use of data products from 2MASS, SDSS, and UKIDSS. 2MASS is a joint project of the University of Massachusetts and the Infrared Processing and Analysis Center/California Institute of Technology, funded by the National Aeronautics and Space Administration and the National Science Foundation. SDSS is funded by the Alfred P. Sloan Foundation, the Participating Institutions, the National Science Foundation, the U.S. Department of Energy, the National Aeronautics and Space Administration, the Japanese Monbukagakusho, the Max Planck Society, and the Higher Education Funding Council for England. UKIDSS uses the Wide Field Camera at the United Kingdom Infrared Telescope atop Mauna Kea, Hawai'i. We are grateful for the efforts of the instrument, calibration, and pipeline teams that have made the UKIDSS data possible. We acknowledge use of the DSS, which were produced at the Space Telescope Science Institute under U.S. Government grant NAG W-2166. The images of these surveys are based on photographic data obtained using the Oschin Schmidt Telescope on Palomar Mountain and the UK Schmidt Telescope. This research has made use of the NASA/IPAC Infrared Science Archive (IRSA), which is operated by the Jet Propulsion Laboratory, California Institute of Technology, under contract with the National Aeronautics and Space Administration. Our research has benefited from the M, L, and T dwarf compendium housed at DwarfArchives.org, whose server was funded by a NASA Small Research Grant, administered by the American Astronomical Society. We are also indebted to the SIMBAD database, operated at CDS, Strasbourg, France. This work is based in part on observations made with the Spitzer Space Telescope, which is operated by the Jet Propulsion Laboratory, California Institute of Technology, under a contract with NASA. Support for this work was provided by NASA through an award issued to program 70062 by JPL/Caltech. This work is also based in part on observations made with the NASA/ESA Hubble Space Telescope, obtained at the Space Telescope Science Institute, which is operated by the Association of Universities for Research in Astronomy, Inc., under NASA contract NAS 5-26555. These observations are associated with program 12330. Support for program 12330 was provided by NASA through a grant from the Space Telescope Science Institute. Some of the spectroscopic data presented herein were obtained at the W. M. Keck Observatory, which is operated as a scientific partnership among the California Institute of Technology, the University of California and the National Aeronautics and Space Administration. The Observatory was made possible by the generous financial support of the W. M. Keck Foundation. In acknowledgement of our observing time at Keck and the IRTF, we further wish to recognize the very significant cultural role and reverence that the summit of Mauna Kea has always had within the indigenous Hawai'ian community. We are most fortunate to have the opportunity to conduct observations from this mountain. We acknowledge use of PAIRITEL, which is operated by the Smithsonian Astrophysical Observatory (SAO) and was made possible by a grant from the Harvard University Milton Fund, the camera loaned from the University 


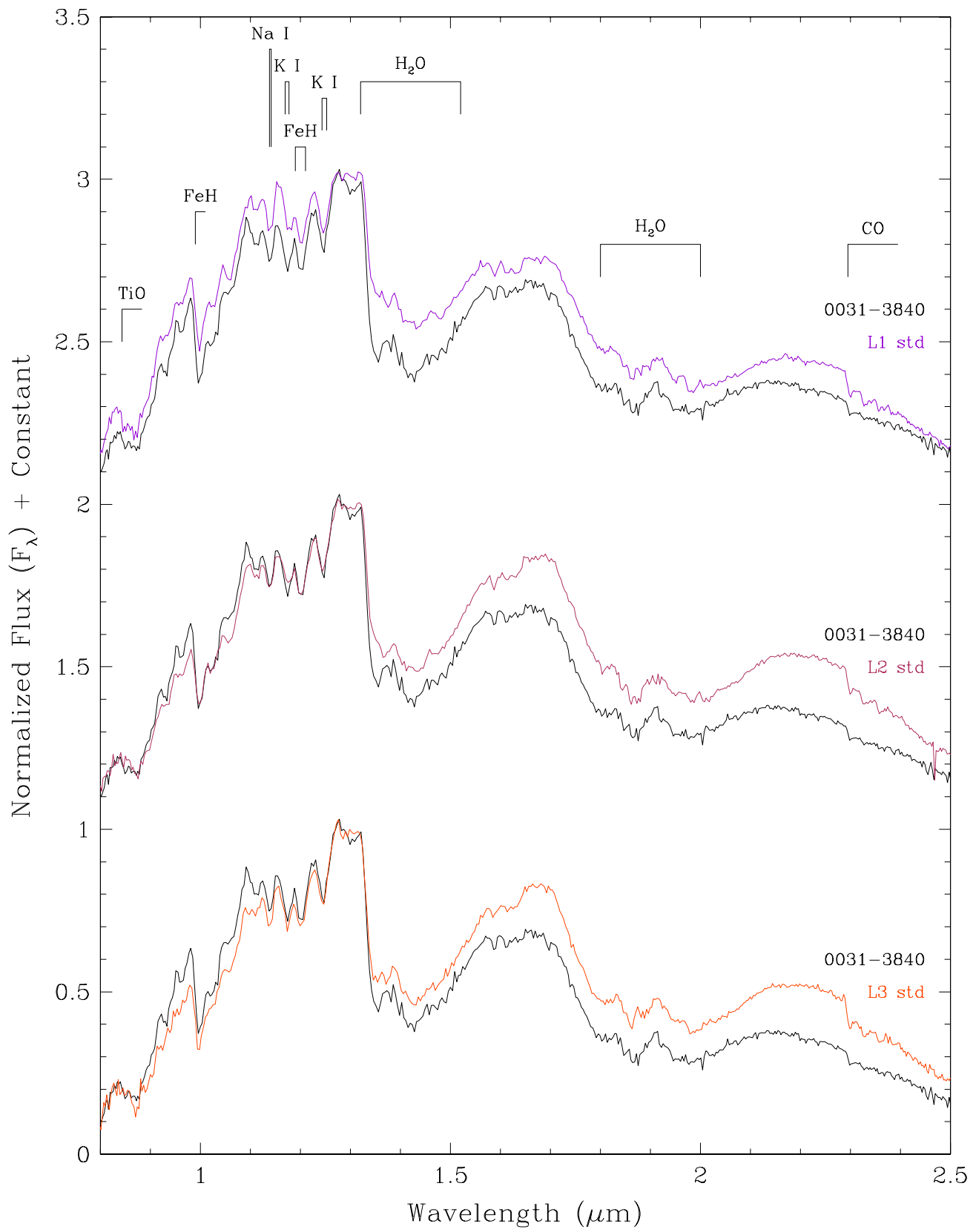

Figure 33. Near-infrared spectrum of WISE 0031-3840 (black) compared to the L1 (dark violet), L2 (maroon) and L3 (orange red) spectral standards from Kirkpatrick et al. (2010). Spectra have been normalized to one at $1.28 \mu \mathrm{m}$ and integral offsets have been added to the $y$-axis values to separate the spectra vertically except where overplotting was intended. Prominent spectral features are marked.

(A color version of this figure is available in the online journal.)

of Virginia, and the continued support of the SAO and UC Berkeley. The PAIRITEL project is supported by NASA Grant NNX10AI28G. We thank Dan Starr, Cullen Blake, Adam Morgan, Adam Miller, and Chris Klein for their assistance. This paper also includes data gathered with the $6.5 \mathrm{~m}$ Magellan Telescopes located at Las Campanas Observatory, Chile. Portions of our Magellan telescope time were granted by the National Optical Astronomy Observatory (NOAO; Proposal ID 2010B0184), through the Telescope System Instrumentation Program (TSIP). TSIP is funded by NOAO, which is operated by the Association of Universities for Research in Astronomy under cooperative agreement with the National Science Foundation. We thank Alan Tokunaga for granting director's discretionary time with IRTF/SpeX for some of the observations presented herein.

\section{APPENDIX A}

\section{NOTES ON SPECIAL OBJECTS}

Notes are given below for objects with unusual spectra, spectrophotometric distance estimates placing them within $10 \mathrm{pc}$ of the Sun, spectral types later than T9, or possible companionship with a nearby object previously cataloged. In the sections below, the spectra of objects are assumed to be normal unless peculiarities are specifically mentioned.

\section{A.1. WISEPC J003119.76-384036.4 $(\mathrm{J}=14.1 \mathrm{mag}$, $W 2=12.0 \mathrm{mag}$ )}

The $J$-band spectrum of this object, which was earlier cataloged as SIPS J0031-3840 and identified to be a nearby star 


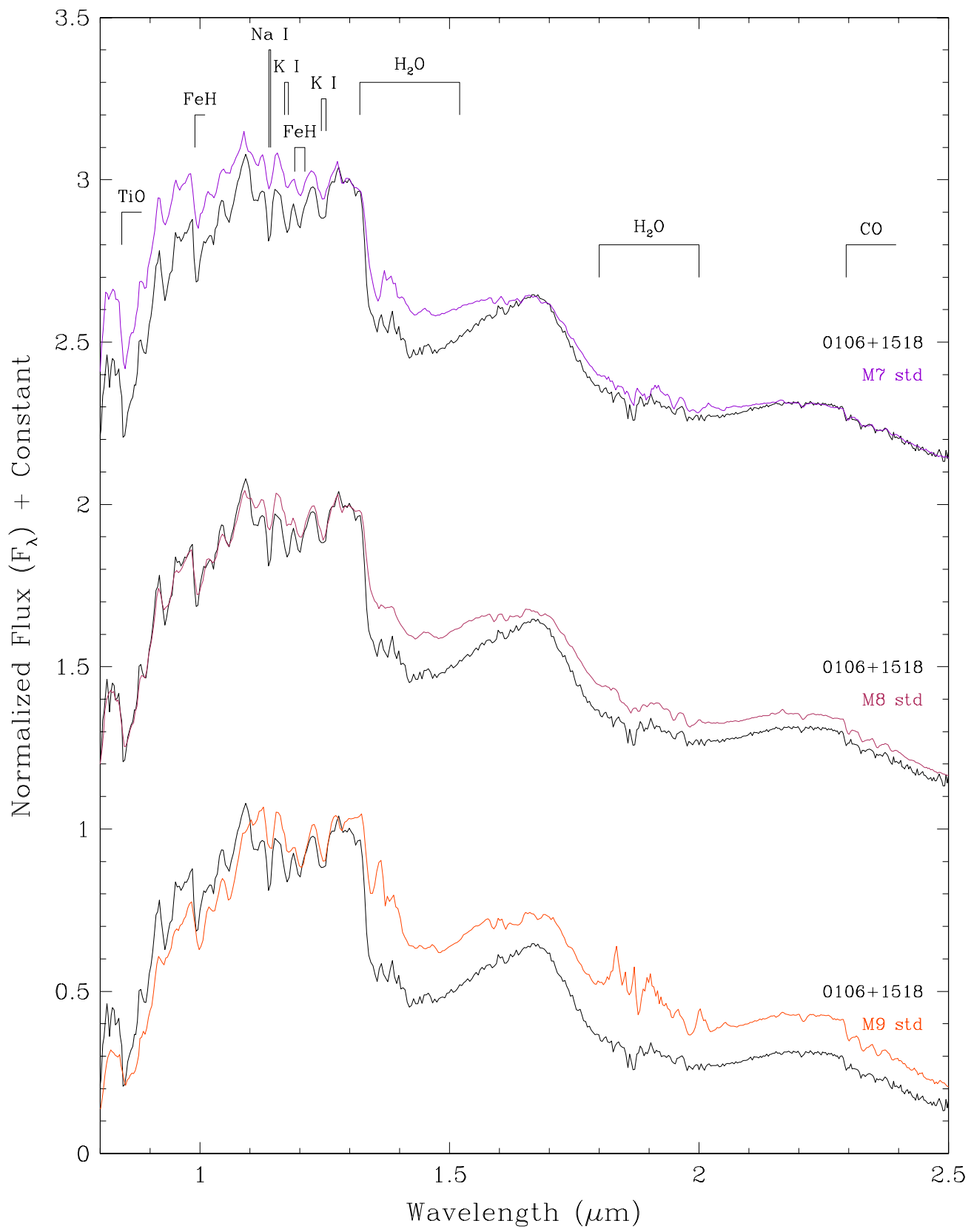

Figure 34. Near-infrared spectrum of WISE 0106+1518 (black) compared to the M7 (dark violet), M8 (maroon) and M9 (orange red) spectral standards from Kirkpatrick et al. (2010). Spectra have been normalized to one at $1.28 \mu \mathrm{m}$ and integral offsets have been added to the $y$-axis values to separate the spectra vertically except where overplotting was intended. Prominent spectral features are marked.

(A color version of this figure is available in the online journal.)

via its high proper motion by Deacon et al. (2005), is a good match to the spectrum of the L2 standard (Figure 33), but the spectrum is much bluer than the standard at $H$ and $K$ bands. Because this source does not exhibit other telltale signs of low metallicity, such as strong hydride bands, we classify it as an "L2 pec (blue)." Martín et al. (2010) give an optical spectral type of L2.5 and do not note any peculiarities that might be attributable to low metallicity, either. Its near-infrared spectral morphology and discrepancy relative to its nearest standard is most similar to the "L1 pec (sl. blue)" object 2MASS J14403186-1303263 shown in Figure 32 of Kirkpatrick et al. (2010). Furthermore, this object has a larger $W 1-W 2$ color than a typical L2 (see Figure 1) and a bluer $J-H$ color (see Figure 5). As discussed in Kirkpatrick et al. (2010), the physical interpretation of these "blue L dwarfs" is not fully known and may differ from object to object. Some blue L dwarfs are blue, for example, because they are composite $\mathrm{L}+\mathrm{T}$ dwarf binaries (e.g., Burgasser 2007). Model fitting suggests that others have thin cloud decks and/or large grains in their atmosphere, though neither seems to be directly attributable to gravity or metallicity effects (Burgasser et al. 2008). Kinematic analysis by Faherty et al. (2009) has shown that the blue L dwarfs have kinematics older than the field L dwarf population, but not nearly as old as that of lowmetallicity M dwarfs. It is possible that some of the blue $\mathrm{L}$ dwarfs may be slightly metal poor, and that even a subtle lowering of the metal abundance in these objects may result in the directly measurable effects on the spectral energy distribution seen here. 


\section{A.2. WISEPC J010637.07+151852.8 ( $\mathrm{J}=14.4 \mathrm{mag}$, $W 2=12.7 \mathrm{mag})$}

Despite the fact that the spectrum of this object matches very well to the $J$-band spectrum of the M8 standard, the $H$ band spectrum is more peaked than that seen in a normal M8, with the $\mathrm{H}_{2} \mathrm{O}$ bands on either side of the $H$ peak being stronger than in the standard (Figure 34). This object, which we classify as "M8 pec," is similar to the peculiar late-M dwarf 2MASS J18284076+1229207 shown in Figure 38 of Kirkpatrick et al. (2010). The cause of the peculiarity is not known, but appears not to be due to low gravity, as there are no peculiarities in the strength of the $\mathrm{FeH}$ bands between 0.9 and $1.3 \mu \mathrm{m}$ when compared to the M8 standard. (See, for example, near-IR spectra of low-gravity late-M dwarfs in Figure 14 of Kirkpatrick et al. 2010.) This object also has a larger $W 1-W 2$ color than a typical M8 (see Figure 1) and a bluer $J-H$ color (see Figure 5). Curiously, this object has a sizable motion- $\mu=0.412 \pm 0.006 \operatorname{arcsec}_{\mathrm{yr}^{-1}}$ - and a tangential velocity of $85.5 \pm 1.3 \mathrm{~km} \mathrm{~s}^{-1}$, suggesting that it may belong to an old population. The peculiar spectroscopic features may be caused in part by a slightly subsolar metallicity. Deacon et al. (2009) also identified this object as the high motion source ULAS2MASS J0106+1518, and their propermotion determination $\left(\mu=0.407 \operatorname{arcsec}^{-1}\right)$ agrees with the one we derive here.

\section{A.3. WISEPC J014807.25-720258.7 $(\mathrm{J}=19.0 \mathrm{mag}$, $W 2=14.6 \mathrm{mag})$}

The near-infrared spectrum of this object, discussed in Cushing et al. (2011), is distinctly later in type than the T9 nearinfrared standard, UGPS J072227.51-054031.2, and is therefore classified as T9.5. Our spectrophotometric distance places it at $12.1 \mathrm{pc}$ (Table 6). This is the only one of our $\geqslant$ T9.5 discoveries not detected in $W 3$ and along with WISE $1738+2732$ is one of only two $\geqslant$ T9.5 dwarfs detected in $W 1$ (Table 2).

\section{A.4. WISEPA J020625.26+264023.6 $(\mathrm{J}=16.5 \mathrm{mag}$, $W 2=12.8 \mathrm{mag}$ )}

The $J$-band spectrum of this object closely matches that of the L9 spectral standard, but the $H$ - and $K$-band portions are much redder than those of the standard L9 (Figure 35). This extremely red color is supported by independent photometry, namely $J-K_{s}=2.007 \pm 0.137 \mathrm{mag}$, from the 2MASS All-Sky Point Source Catalog. This color is somewhat redder than the mean $J-K_{s}$ color, $\sim 1.78 \mathrm{mag}$, of very late L's (Figure 14 of Kirkpatrick 2008). Because of its spectral peculiarity, we classify this object as "L9 pec (red)," and add it to the growing list of L dwarfs that appear red for reasons not obviously attributable to low gravity (see Table 6 of Kirkpatrick et al. 2010). The underlying physical cause for these "red L dwarfs" is not known, although two have been studied in detail by Looper et al. (2008). Using the small sample of red L dwarfs then known, Kirkpatrick et al. (2010) found that these objects, unlike young, low-gravity L dwarfs that are also redder than spectral standards of the same type, appear to have older kinematics than that of the field L dwarf population.

$$
\begin{gathered}
\text { A.5. WISEPA J025409.45+022359.1 }(\mathrm{J}=15.9 \mathrm{mag}, \\
W 2=12.7 \mathrm{mag})
\end{gathered}
$$

This object is a nearby T8 dwarf at a spectrophotometric distance of $d=6.9$ pc. Our astrometry (Table 6) over a $10.4 \mathrm{yr}$ baseline indicates a high motion of $\mu=2.546 \pm 0.046$ arcsec $\mathrm{yr}^{-1}$ and a large tangential velocity of $83.3 \pm 1.5 \mathrm{~km} \mathrm{~s}^{-1}$. Our preliminary trigonometric parallax measurement (Table 7) places this object at $6.1_{-1.4}^{+2.3} \mathrm{pc}$, in excellent agreement with the spectrophotometric estimate.

$$
\begin{gathered}
\text { A.6. WISEPA J031325.96+780744.2 }(\mathrm{J}=17.7 \mathrm{mag}, \\
W 2=13.2 \mathrm{mag})
\end{gathered}
$$

This T8.5 dwarf has a spectrophotometric distance estimate of only $8.1 \mathrm{pc}$.

$$
\begin{gathered}
\text { A.7. WISEPA J041022.71+150248.5 }(\mathrm{J}=19.3 \mathrm{mag}, \\
W 2=14.2 \mathrm{mag})
\end{gathered}
$$

The near-infrared spectrum of this object, discussed in Cushing et al. (2011), is classified as Y0. Our distance estimate places it 9.0 pc from the Sun, and our measurement of the proper motion indicates that it may also be a high mover$\mu=2.429 \pm 0.334$ arcsec $\mathrm{yr}^{-1}$ —although the error bar is large (Table 6). As with most of the other Y dwarf discoveries, this object is detected by WISE only in bands W2 and W3 and not in $W 1$ or $W 4$ (Table 2).

\section{A.8. WISEPA J044853.29-193548.5 ( $\mathrm{J}=17.0 \mathrm{mag}$, $W 2=14.2 \mathrm{mag})$}

The depths of the $\mathrm{H}_{2} \mathrm{O}$ and $\mathrm{CH}_{4}$ absorption bands in the $J$ - and $H$-band spectra of this object best fit the T5 standard; however, the spectrum shows excess flux in the $Y$ band around 0.95 to $1.10 \mu \mathrm{m}$ and a flattening of the entire $K$ band spectrum (Figure 36). We therefore classify this object as a "T5 pec." Excess flux at $Y$ band and a flattening at $K$ have also been noted in Burgasser et al. (2006a) for the T6 pec dwarf 2MASS J09373487+2931409, which may be slightly metal-poor $([\mathrm{M} / \mathrm{H}] \approx-0.5$ to -0.1$)$ based on fits to model spectra. Burgasser et al. (2011a) and Burgasser et al. (2010a) have noted the same peculiarities in the spectrum of the T7.5 dwarf ULAS J141623.94+134836.3 (Scholz 2010a; Burningham et al. 2010a), which is a common propermotion companion to the nearby, late-L dwarf SDSS J141624.08+134826.7 (Schmidt et al. 2010; Bowler et al. 2010). The latter is classified by Kirkpatrick et al. (2010) as sdL7 and by Burningham et al. (2010a) as d/sdL7. Given that the two objects are presumably coeval, it can be assumed that they have the same metallicity and that the peculiar features in the spectrum of ULAS J141623.94+134836.3 - and by extension, WISE 0448-1935-are caused by a metal content below solar. The high motion of WISE $0448-1935-\mu=1.168 \pm 0.029$ arcsec $\mathrm{yr}^{-1}$, which translates into a tangential velocity of $118.5 \pm$ $2.9 \mathrm{~km} \mathrm{~s}^{-1}$ for an estimated distance of $21.4 \mathrm{pc}$-also indicates that this object belongs to an old kinematic population.

\section{A.9. WISEPA J045853.89+643452.9 $(\mathrm{J}=18.3 \mathrm{mag}$, $W 2=13.0 \mathrm{mag})$}

This object was discussed in detail by Mainzer et al. (2011). Our distance estimate of $7.3 \mathrm{pc}$ assumes a single source, but analysis of laser guide star adaptive optics data from Gelino et al. (2011) indicates that the source is a double in which the components have $\Delta J \approx \Delta H \approx 1 \mathrm{mag}$. Individual magnitudes are measured as $J_{A}=17.50 \pm 0.09, J_{B}=18.48 \pm 0.12$ and $H_{A}=17.81 \pm 0.13, H_{B}=18.81 \pm 0.17$ on the MKO filter system. Gelino et al. (2011) suggest an actual distance to the system of $12.3 \pm 2.3 \mathrm{pc}$ and individual spectral types of T8.5 and T9. 


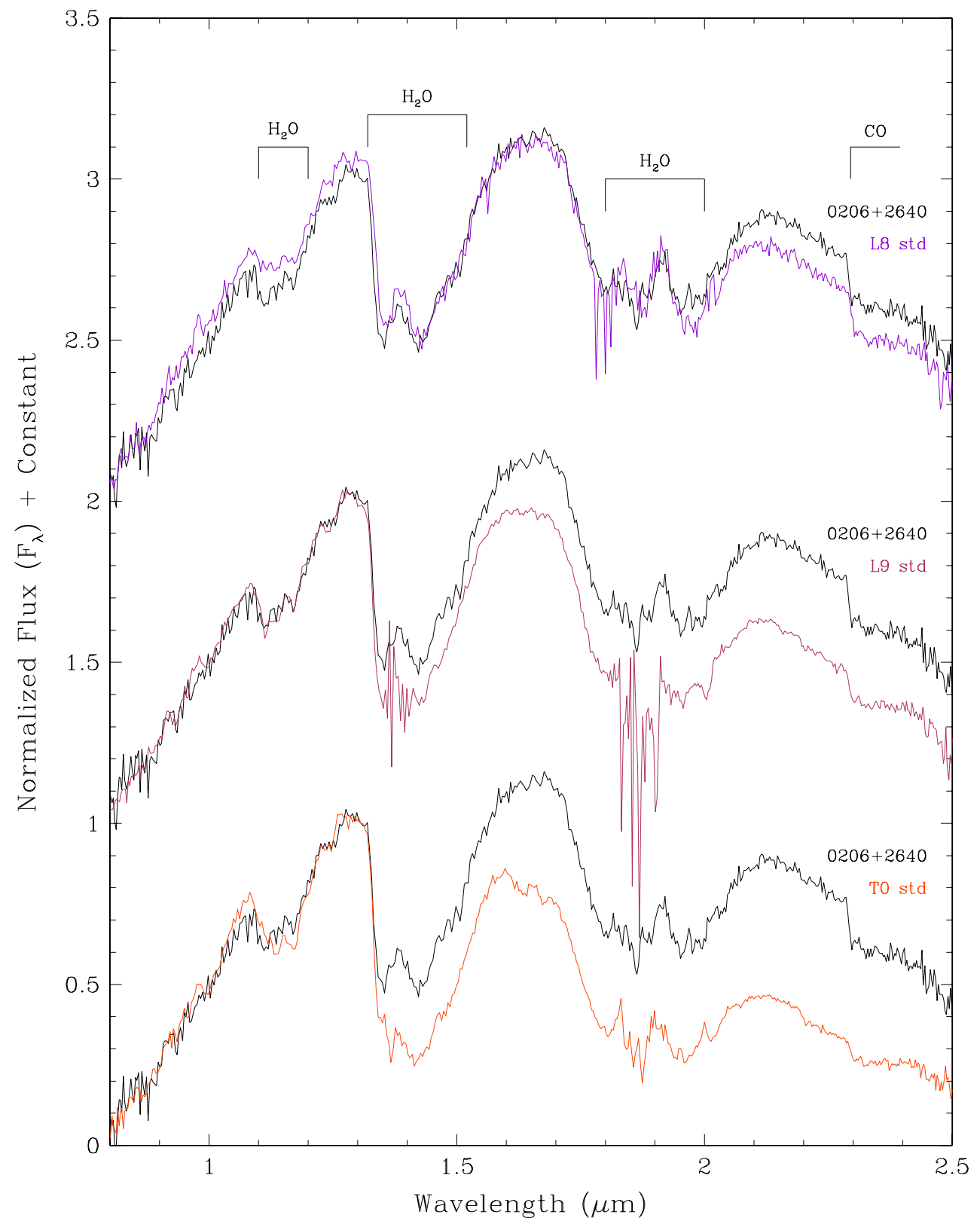

Figure 35. Near-infrared spectrum of WISE 0206+2640 (black) compared to the L8 (dark violet), L9 (maroon), and T0 (orange red) spectral standards from Kirkpatrick et al. (2010) and Burgasser et al. (2006b). Spectra have been normalized to one at $1.28 \mu \mathrm{m}$ and integral offsets have been added to the $y$-axis values to separate the spectra vertically except where overplotting was intended. Prominent spectral features are marked.

(A color version of this figure is available in the online journal.)

\section{A.10. WISEPA J052536.33+673952.3 $(\mathrm{J}=17.5 \mathrm{mag}$, W2 = 14.9 mag)}

The $J$-band spectrum of this object best fits the T6 standard, but there are discrepancies at $Y$ and $K$ bands. At $Y$ band the spectrum of WISE $0525+6739$ shows excess flux relative to the standard, and at $K$ band the spectrum shows less flux relative to the standard (Figure 37). We therefore classify this object as a "T6 pec." The physical cause, as discussed above for WISE 0448-1935, may be low metal content.

\section{A.11. WISEPA J052844.51-330823.9 $(\mathrm{J}=16.7 \mathrm{mag}$ $W 2=14.5 \mathrm{mag})$}

The $J$-band spectrum of this object best fits the T7 standard, but there are discrepancies at the $Y$ and $K$ bands. At the $Y$ band the spectrum of WISE 0528-3308 shows excess flux relative to the standard, and at the $K$ band the spectrum shows less flux relative to the standard (Figure 38 ). We therefore classify this object as a "T7 pec." The physical cause, as discussed above for both WISE 0448-1935 and WISE 0525+6739, may be low metal content.

$$
\begin{gathered}
\text { A.12. WISEPC J083641.12-185947.2 (J= unknown, } \\
W 2=15.0 \text { mag })
\end{gathered}
$$

The $J$-band spectrum of this object best fits the T8 standard, but there are major discrepancies at the $Y$ and $K$ bands. At the $Y$ band the spectrum of WISE 0836-1859 shows excess flux relative to the standard, and at the $K$ band the spectrum shows less flux relative to the standard (Figure 39). We therefore classify this object as a "T8 pec." Several other objects discussed in this section-WISE 0448-1935, WISE 0525+6739, WISE 0528-3308, WISE 1436-1814, WISE 2134-7137, and WISE 


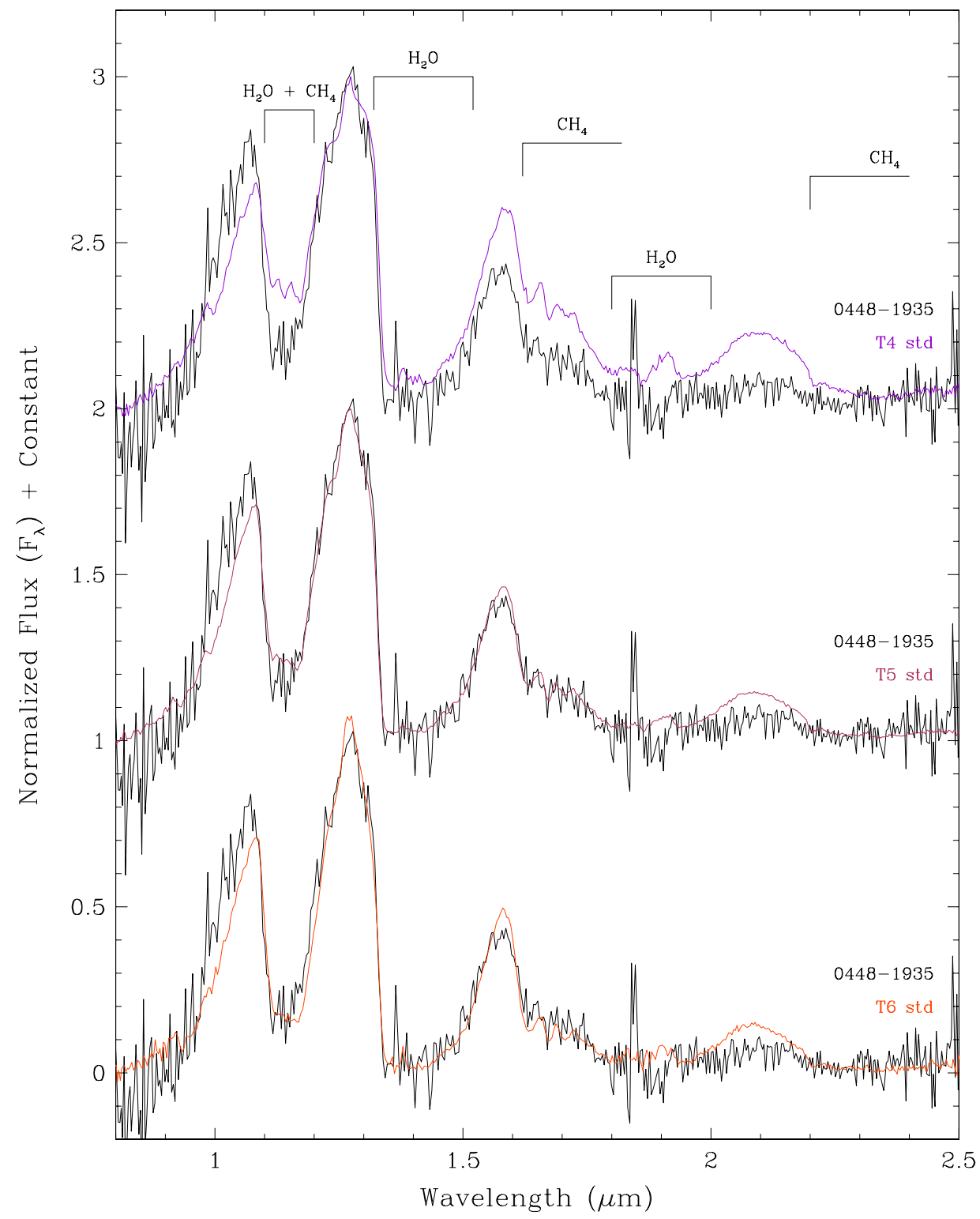

Figure 36. Near-infrared spectrum of WISE 0448-1935 (black) compared to the T4 (dark violet), T5 (maroon), and T6 (orange red) spectral standards from Burgasser et al. (2006b). Spectra have been normalized to one at $1.28 \mu \mathrm{m}$ and integral offsets have been added to the $y$-axis values to separate the spectra vertically except where overplotting was intended. Prominent spectral features are marked.

(A color version of this figure is available in the online journal.)

2325-4105-have similar $Y$ - and $K$-band discrepancies, which may result from low metal content, but none are as severe as the discrepancies in this object. Unlike in those objects, the $H$-band flux in WISE 0836-1859 is markedly lower than the closest matching standard at $J$, making this object the most peculiar one of the group, and perhaps also the most metal poor.

Ideally, we would like to study the frequency of these metalpoor T dwarfs to see if the numbers found are what star formation theory would predict for an old, field population. Unfortunately, we do not have cold models across a large grid of metallicities with which to determine the $[\mathrm{M} / \mathrm{H}]$ values of our spectra. We suspect that in cool objects such as these, slight changes in the metal content can have profound effects on the emergent spectra. As a result, we may be able to detect via spectroscopy smaller changes in $[\mathrm{M} / \mathrm{H}]$ for $\mathrm{T}$ dwarfs than are possible in hotter stars due to the richness of molecular species and the important role of condensation in determining the absorbing species of colder objects. In other words, the metal content of these $\mathrm{T}$ dwarfs may not be too different from solar. As discussed above for WISE 0448-1935, the T7.5 dwarf ULAS $\mathrm{J} 141623.94+134836.3$ shows peculiarities in its spectrum that are similar to the ones seen in these unusual WISE T dwarfs, yet models with a subsolar abundance of only $[\mathrm{M} / \mathrm{H}]=-0.3$ provide good fits to the emergent spectrum of that object (Burgasser et al. 2010a).

$$
\begin{gathered}
\text { A.13. WISEPC J112254.73+255021.5 ( } \mathrm{J}=16.7 \mathrm{mag}, \\
W 2=14.0 \mathrm{mag})
\end{gathered}
$$

This object, a normal T6 dwarf, lies 265 arcsec away from the nearby M5 V star LHS 302 (GJ 3657). Our spectrophotometric distance estimate for WISE $1122+2550$ (16.9 pc; Table 6) is very similar to the distance of $17.2 \mathrm{pc}$ obtained via trigonometric parallax $(0.0581 \pm 0.0039$ arcsec $)$ for LHS 302 (Dahn et al. 1988). Moreover, the right ascension and declination components of the proper motion of WISE $1122+2550$ are measured by us to be $-0.954 \pm 0.016$ and $-0.276 \pm 0.018$ 


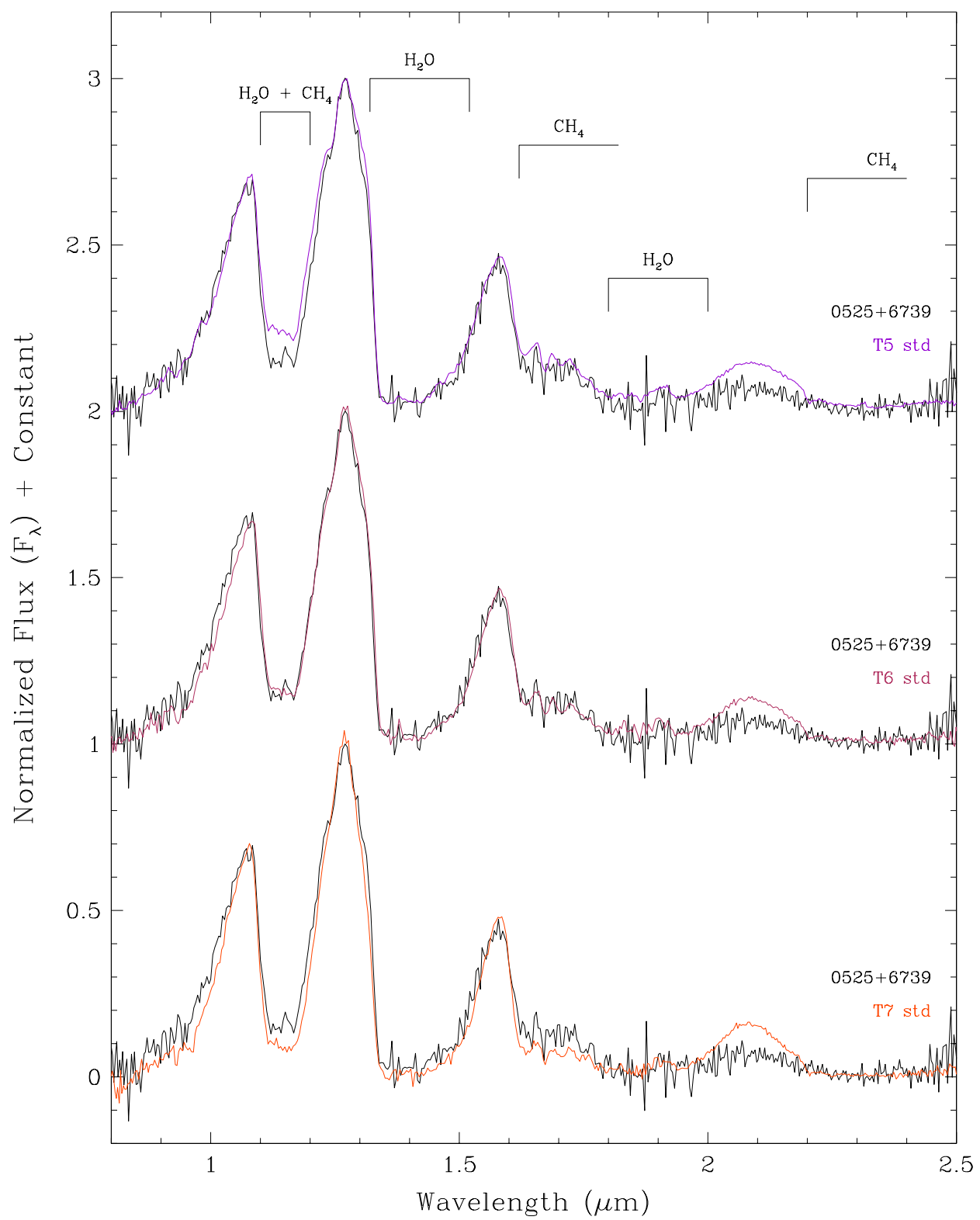

Figure 37. Near-infrared spectrum of WISE 0525+6739 (black) compared to the T5 (dark violet), T6 (maroon), and T7 (orange red) spectral standards from Burgasser et al. (2006b). Spectra have been normalized to one at $1.28 \mu \mathrm{m}$ and integral offsets have been added to the $y$-axis values to separate the spectra vertically except where overplotting was intended. Prominent spectral features are marked.

(A color version of this figure is available in the online journal.)

$\operatorname{arcsec} \mathrm{yr}^{-1}$, respectively, which are only $\sim 3 \sigma$ different from those measured for LHS $302\left(-1.002 \pm 0.001 \operatorname{arcsec}^{\mathrm{yr}^{-1}}\right.$ and $-0.330 \pm 0.001 \operatorname{arcsec}^{-1}{ }^{-1}$; Dahn et al. 1988). If these two objects are a common proper-motion binary, the projected separation between them is $\sim 4500 \mathrm{AU}$.

Other stellar + substellar binaries of large separation are known. Examples are the Gliese 570 system comprised of K4 V, $\mathrm{M} 1.5 \mathrm{~V}+\mathrm{M} 3 \mathrm{~V}$, and $\mathrm{T} 7.5$ components with the latter having a projected separation of $1500 \mathrm{AU}$ from the K star; the Gliese 584 system comprised of G1 V + G3 V and L8 components with a projected separation of $3600 \mathrm{AU}$ (Kirkpatrick et al. 2001); the Gliese 417 system comprised of G0 V and L4.5 components with a projected separation of $2000 \mathrm{AU}$ (Kirkpatrick et al. 2001); the Gliese 618.1 system comprised of M0 V and L2.5 components with a projected separation of $1000 \mathrm{AU}$ (Wilson et al. 2001); the HD 89744 system comprised of F7 IV-V and L0 components with a projected separation of $2500 \mathrm{AU}$ (Wilson et al. 2001); and the HD 2057 system comprised of F8 and L4 components with a projected separation of 7000-9000 AU (Cruz et al. 2007). Each of these systems, however, has a more massive primary than the one discussed here. Assuming that LHS 302 has a mass of $\sim 0.2 M_{\odot}$ in concert with other M5 dwarfs (see López-Morales 2007), then our projected separation of 4500 AU falls well outside the $\Delta_{\max }=10^{3.33 M_{\text {tot }}+1.1}=58$ AU stability limit suggested empirically by Reid et al. (2001). This suggests that WISE $1122+2550$ and LHS 302 are either physically unbound while sharing a common proper motion or are totally unassociated.

$$
\begin{gathered}
\text { A.14. WISEPC J115013.88+630240.7 }(J=17.7 \mathrm{mag}, \\
\text { W2 }=13.4 \mathrm{mag})
\end{gathered}
$$

Our distance estimate places this T8 dwarf $9.6 \mathrm{pc}$ from the Sun. 


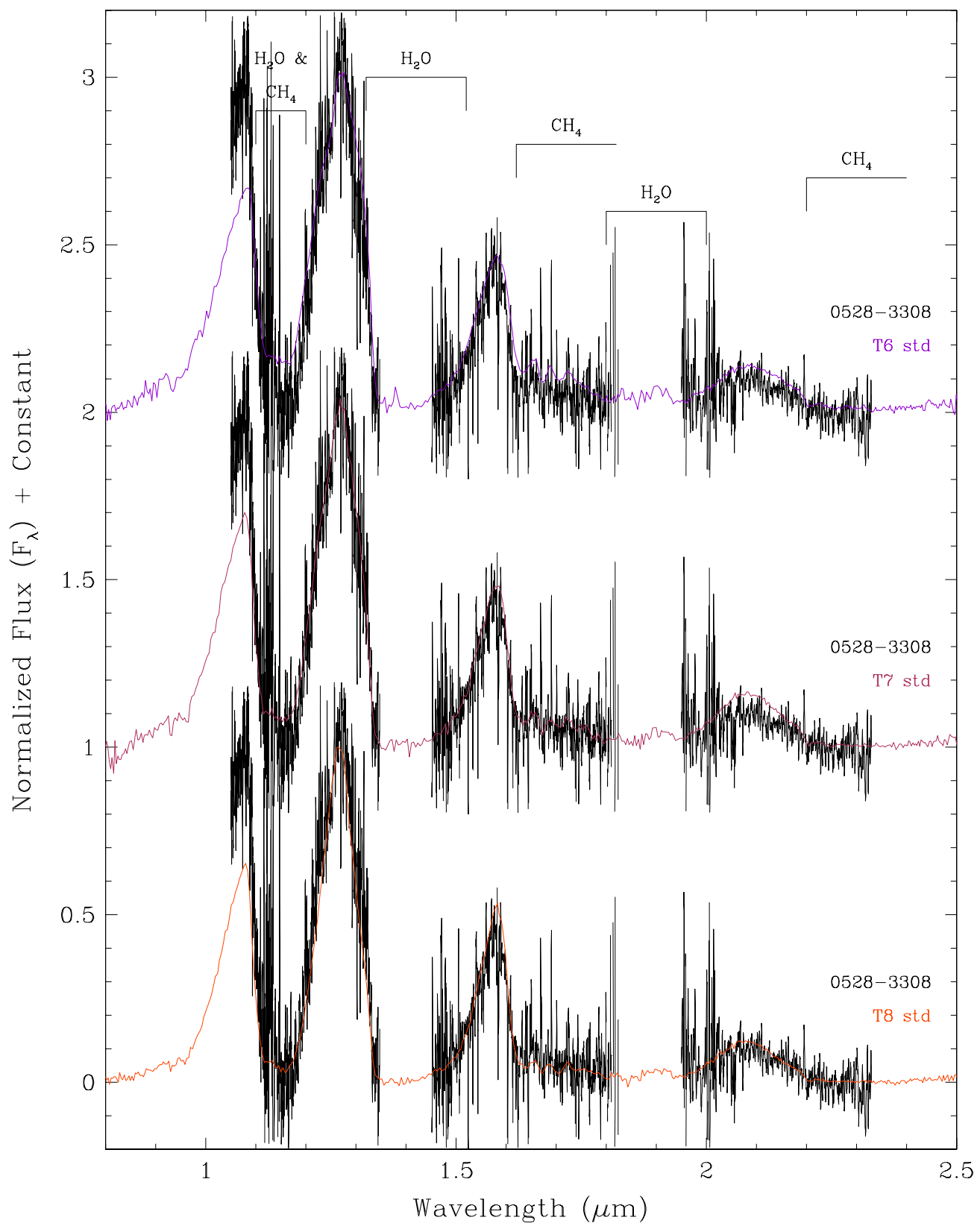

Figure 38. Near-infrared spectrum of WISE 0528-3308 (black) compared to the T6 (dark violet), T7 (maroon), and T8 (orange red) spectral standards from Burgasser et al. (2006b). Spectra have been normalized to one at $1.28 \mu \mathrm{m}$ and integral offsets have been added to the $y$-axis values to separate the spectra vertically except where overplotting was intended. Prominent spectral features are marked. The spectrum of WISE 0528-3308 has been smoothed with a 5 pixel boxcar.

(A color version of this figure is available in the online journal.)

\section{A.15. WISEPC J121756.91+162640.2 $(J=17.8 \mathrm{mag}$, $W 2=13.1 \mathrm{mag}$ )}

Our distance estimate places this T9 dwarf $6.7 \mathrm{pc}$ from the Sun. Our measurement of $\mu=1.765 \pm 0.388 \operatorname{arcsec}^{-1} \mathrm{r}^{-1}$, based on astrometry covering only $0.7 \mathrm{yr}$, may also indicate a high proper motion, but the uncertainty in this value is very large.

$$
\begin{gathered}
\text { A.16. WISEPC J131141.91+362925.2 }(J=15.5 \mathrm{mag}, \\
W 2=13.1 \mathrm{mag})
\end{gathered}
$$

The near-infrared spectrum of this source is an excellent match to the L5 standard at $J$ band. At longer wavelengths, however, the spectrum is considerably bluer, as shown in Figure 40 . There is no evidence in the $J$ band that this source has a low-metallicity, and therefore that the $H$ and $K$ bands are being suppressed by the relatively stronger collision-induced absorption by $\mathrm{H}_{2}$ one would expect in a metal-starved atmosphere. The notch near $1.62 \mu \mathrm{m}$ in the top of the $H$-band peak is very similar to the interesting feature noted by Burgasser (2007) in the spectrum of SDSS J080531.84+481233.0, which those authors claim is an unresolved mid-L + mid-T binary. The $H$-band notch is also seen by Burgasser et al. (2011b) in the spectrum of 2MASS J13153094-2649513, which those authors have successfully split, via high-resolution imaging and spectroscopy, into an L5 + T7 double. We classify WISE $1311+3629$ as an "L5 blue" and note that its peculiar features may be caused by unresolved binarity as well. Our formal fits to synthetic binaries (see Burgasser 2007 for details) show that the most likely spectral types of the two components are $\mathrm{L} 3.5 \pm 0.7$ and $\mathrm{T} 2 \pm 0.5$ with estimated relative magnitudes of $\Delta J=1.4 \pm 0.2, \Delta H=1.7 \pm 0.3$, 


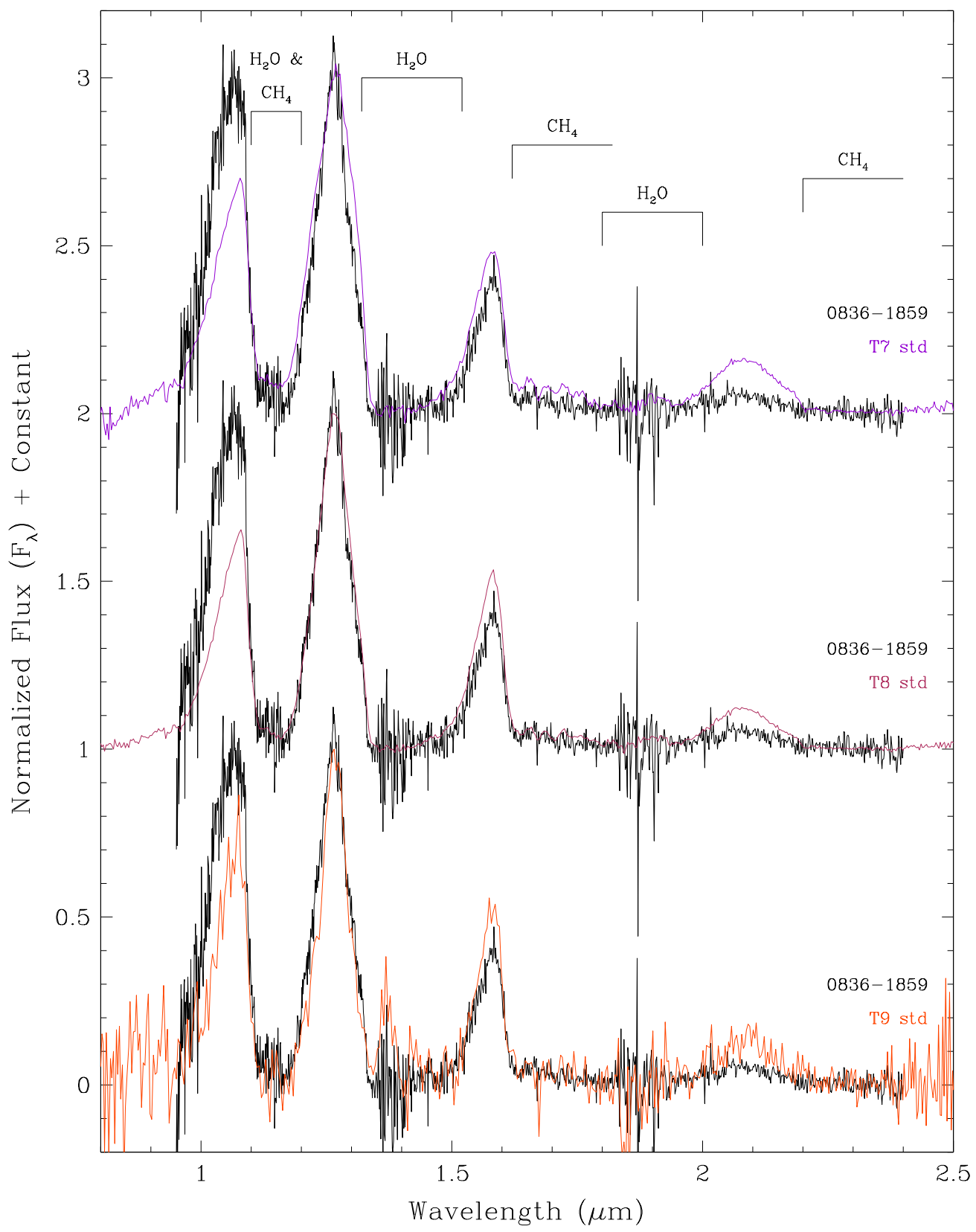

Figure 39. Near-infrared spectrum of WISE 0836-1859 (black) compared to the T7 (dark violet), $\mathrm{T} 8$ (maroon), and T9 (orange red) spectral standards from Burgasser et al. (2006b) and M. C. Cushing et al. (submitted). Spectra have been normalized to one at $1.28 \mu \mathrm{m}$ and integral offsets have been added to the $y$-axis values to separate the spectra vertically except where overplotting was intended. Prominent spectral features are marked.

(A color version of this figure is available in the online journal.)

and $\Delta K=2.2 \pm 0.3 \mathrm{mag}$. This object would be an excellent target for high-resolution imaging. We note that this object was also identified as a brown dwarf candidate by Zhang et al. (2009) and given the designation SDSS J131142.11+362923.9.

\section{A.17. WISEPC J140518.40+553421.4 $(\mathrm{J}=20.2 \mathrm{mag}$ $W 2=14.1 \mathrm{mag})$}

This object is tentatively classified as $\mathrm{Y} 0$ (pec?) by Cushing et al. (2011), who describe its spectral features and derived physical parameters. We estimate a distance of $8.6 \mathrm{pc}$ and find a high proper motion of $2.693 \pm 0.398 \operatorname{arcsec~} \mathrm{rr}^{-1}$ and high tangential velocity of $109.8 \pm 16.2 \mathrm{~km} \mathrm{~s}^{-1}$. As with most of the other Y dwarf discoveries, this object is detected by WISE only in bands $W 2$ and $W 3$ and not in $W 1$ or $W 4$ (Table 2).

\section{A.18. WISEPA J143602.19-181421.8 ( $J=$ unknown, $W 2=14.7 \mathrm{mag})$}

The $J$-band spectrum of this object best fits the T8 standard, but there are discrepancies at the $Y$ and $K$ bands. At the $Y$ band the spectrum of WISE 1436-1814 shows excess flux relative to the standard, and at the $K$ band the spectrum shows less flux relative to the standard (Figure 41). We therefore classify this object as a "T8 pec." The physical cause, as discussed above for several other objects, may be low metal content.

\section{A.19. WISEPC J150649.97+702736.0 $(\mathrm{J}=13.6 \mathrm{mag}$ $W 2=11.3 \mathrm{mag})$}

This T6 dwarf is estimated to fall only $4.9 \mathrm{pc}$ from the Sun. Its large motion $\left(\mu=1.388 \pm 0.131 \operatorname{arcsec} \mathrm{yr}^{-1}\right)$ placed it nearly 


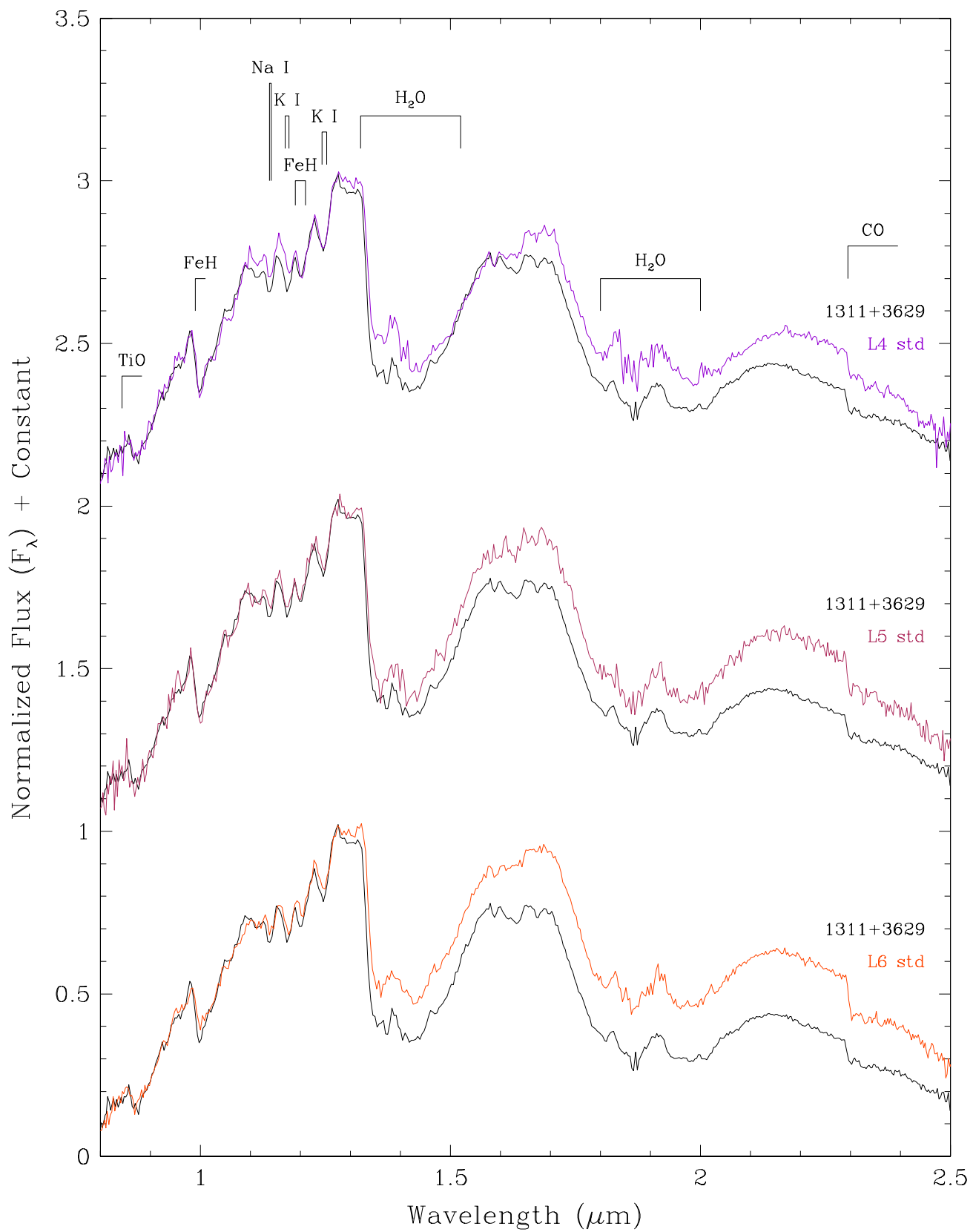

Figure 40. Near-infrared spectrum of WISE 1311+3629 (black) compared to the L4 (dark violet), L5 (maroon) and L6 (orange red) spectral standards from Kirkpatrick et al. (2010). Spectra have been normalized to one at $1.28 \mu \mathrm{m}$ and integral offsets have been added to the $y$-axis values to separate the spectra vertically except where overplotting was intended. Prominent spectral features are marked.

(A color version of this figure is available in the online journal.)

in front of a star, now to the southeast, of similar near-infrared brightness during the 2MASS survey (see Figure 4.12), and this confusion led to the source having been missed in photometric searches of the 2MASS Point Source Catalog.

$$
\begin{gathered}
\text { A.20. WISEPA J154151.66-225025.2 }(J=21.2 \mathrm{mag}, \\
W 2=14.0 \mathrm{mag})
\end{gathered}
$$

The near-infrared spectrum of this object, discussed in Cushing et al. (2011), is classified as Y0 because of its similarity to the spectrum of the Y0 near-infrared standard, WISE $1738+2732$. As with most of the other Y dwarf discoveries, this object is detected by WISE only in bands $W 2$ and $W 3$ and not in $W 1$ or $W 4$ (Table 2). In contrast to our crude spectrophotometric distance estimate from Table 6 of $8.2 \mathrm{pc}$, we measure a trigonometric parallax placing it at $2.8_{-0.6}^{+1.3} \mathrm{pc}$ (Table 7), along with a proper motion of $\mu=0.81 \pm 0.34$ arcsec $\mathrm{yr}^{-1}$. This parallax result is significant only at the $3 \sigma$ level and is measured only over a $1.2 \mathrm{yr}$ baseline, so it should be treated as preliminary only. Nonetheless, if confirmed, this distance would place WISE 1541-2250 as the seventh closest stellar system to the Sun after the $\alpha$ Centauri system $(d=1.3 \mathrm{pc}$; van Leeuwen 2007), Barnard's Star $(d=1.8$ pc; van Leeuwen 2007), Wolf 359 ( $d=2.4 \mathrm{pc}$; van Altena et al. 2001), Lalande $21185(d=2.5 \mathrm{pc}$; van Leeuwen 2007), Sirius $\mathrm{AB}(d=2.6 \mathrm{pc}$; van Leeuwen 2007), and L 726-8 AB (also known as BL Ceti and UV Ceti, $d=2.7 \mathrm{pc}$; van Altena et al. 2001). The measured distance implies absolute magnitudes of $M_{J}=23.9 \pm 0.8$ and $M_{H}=23.8 \pm 0.9 \mathrm{mag}$ on the MKO filter system and $M_{W 2}=16.7 \pm 0.7 \mathrm{mag}$. This indicates a rapid dimming at 


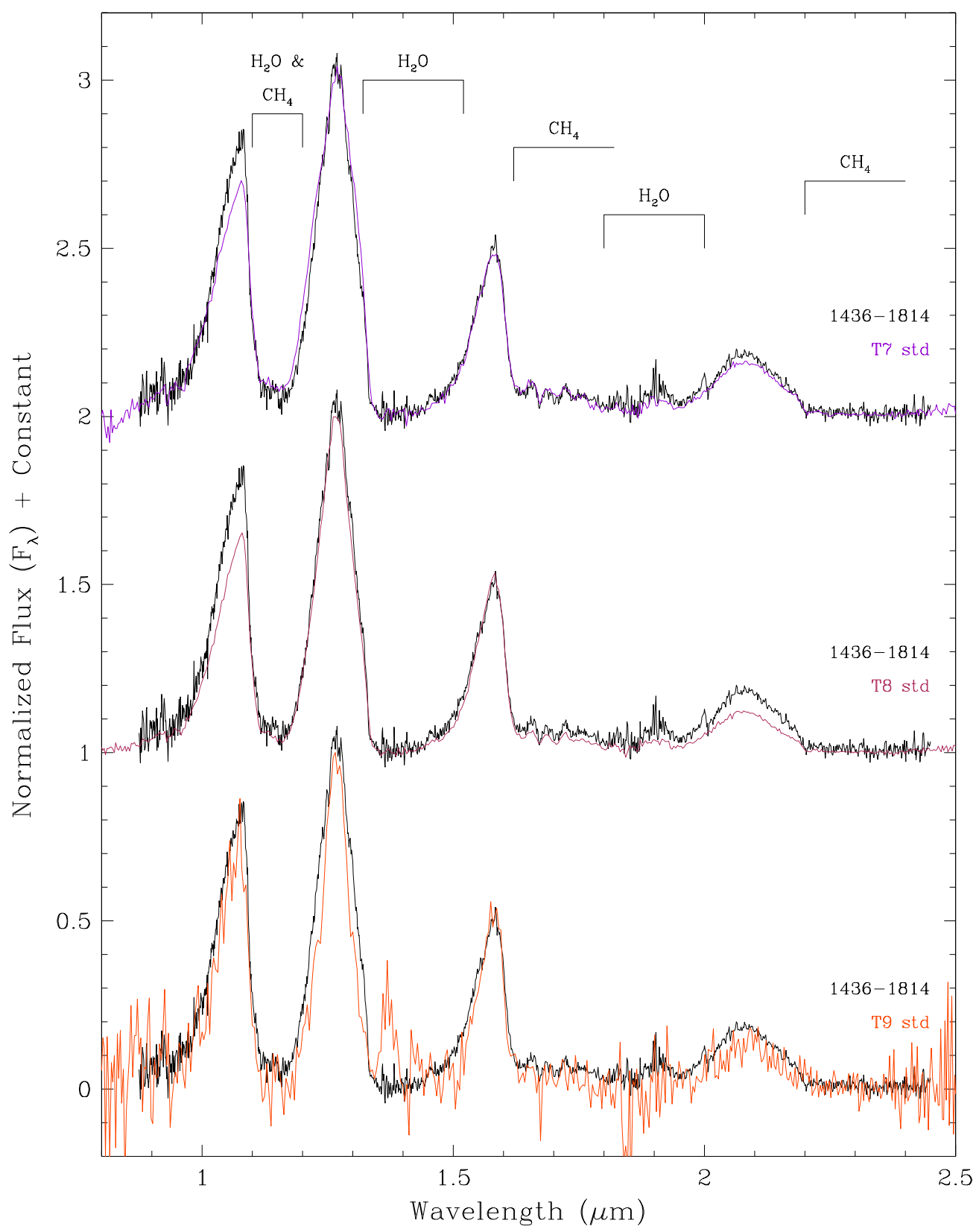

Figure 41. Near-infrared spectrum of WISE 1436-1814 (black) compared to the T7 (dark violet), T8 (maroon), and T9 (orange red) spectral standards from Burgasser et al. (2006b) and Cushing et al. (2011). Spectra have been normalized to one at $1.28 \mu \mathrm{m}$ and integral offsets have been added to the $y$-axis values to separate the spectra vertically except where overplotting was intended. Prominent spectral features are marked.

(A color version of this figure is available in the online journal.)

those wavelengths in just a single spectral subclass from T9 to Y0 (see, e.g., Figures 29 and 31).

\section{A.21. WISEPA J164715.59+563208.2 ( $=16.6 \mathrm{mag}$, $W 2=13.1 \mathrm{mag})$}

The $J$-band spectrum of this object best fits the L9 standard, but there is excess flux at $H$ and particularly $K$ relative to the standard itself (Figure 42). We therefore classify this object as an "L9 pec (red)." This object adds to a growing list of red L dwarfs whose red colors cannot obviously be attributed to low gravity. It becomes the seventh example of this class, which now includes 2MASS J21481633+4003594 and 2MASS J18212815+1414010 from Looper et al. (2008); 2MASS J13313310+3407583, 2MASS J23174712-4838501, and 2MASS J23512200+3010540 from Kirkpatrick et al.
(2010); and WISE 0206+2640 from above. As mentioned in Kirkpatrick et al. (2010), the kinematics of the first five examples suggest that these objects derive from an old population, making them distinct from the red $\mathrm{L}$ dwarfs that have low-gravity spectral signatures and young kinematics. We note, however, that this object has a low tangential velocity of only $28.1 \pm 1.2 \mathrm{~km} \mathrm{~s}^{-1}$. However, this velocity assumes our spectrophotometric distance estimate of $20.2 \mathrm{pc}$, and our preliminary astrometric measurements over an $11.8 \mathrm{yr}$ baseline indicate a closer distance of $8.6_{-1.7}^{+2.9} \mathrm{pc}$, along with a motion of $\mu=0.293 \pm 0.012 \operatorname{arcsec} \mathrm{yr}^{-1}$. Continued astrometric monitoring of this object is needed to see if this closer distance is confirmed, as this would provide another clue in deciphering the physical nature of this rare class of red (non-low- $g$ ) L dwarfs. 


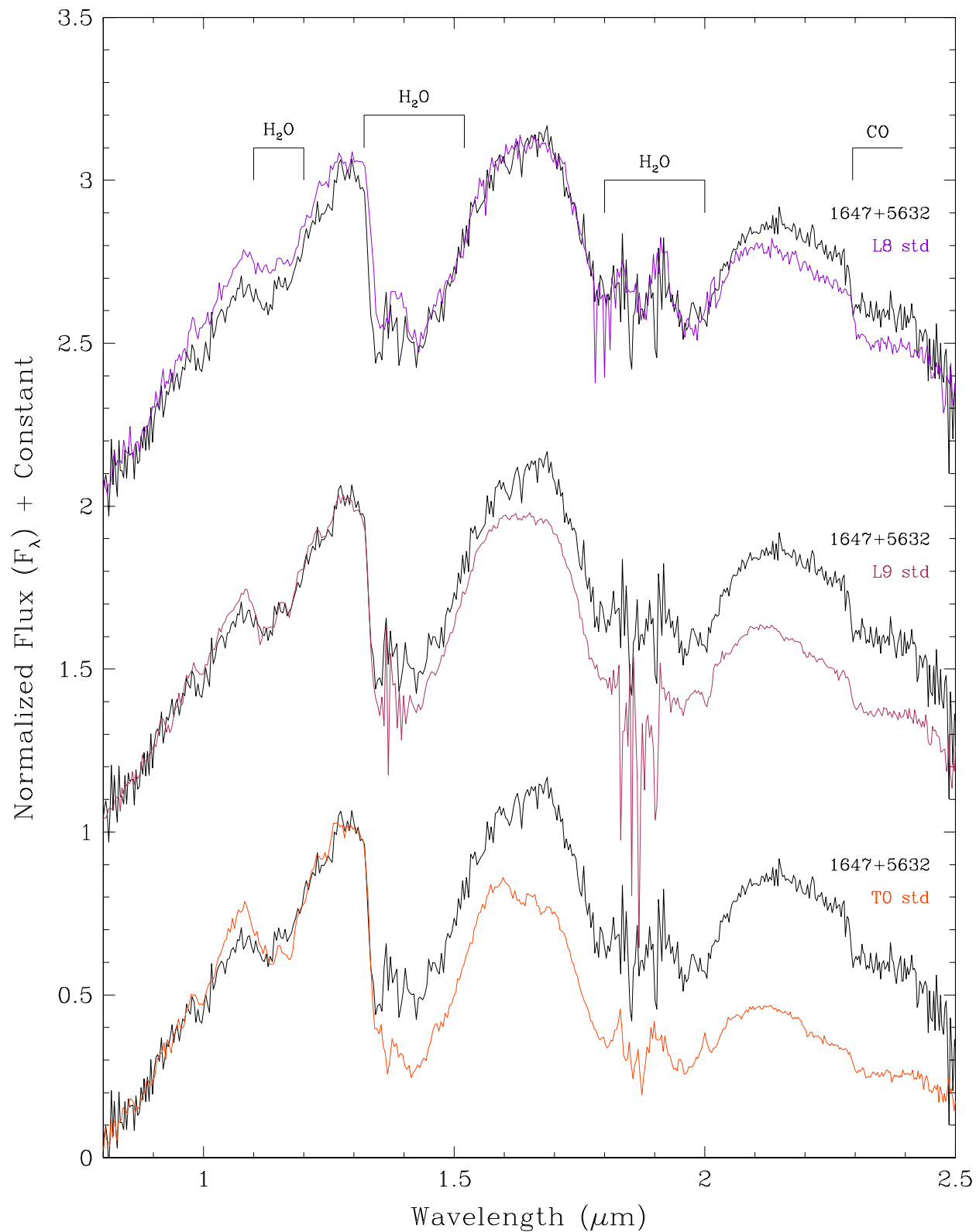

Figure 42. Near-infrared spectrum of WISE 1647+5632 (black) compared to the L8 (dark violet), L9 (maroon), and T0 (orange red) spectral standards from Kirkpatrick et al. (2010) and Burgasser et al. (2006b). Spectra have been normalized to one at $1.28 \mu \mathrm{m}$ and integral offsets have been added to the $y$-axis values to separate the spectra vertically except where overplotting was intended. Prominent spectral features are marked.

(A color version of this figure is available in the online journal.)

\section{A.22. WISEPA J173835.53+273258.9 $(\mathrm{J}=19.5 \mathrm{mag}$, W2 $=14.5 \mathrm{mag})$}

Cushing et al. (2011) propose this object as the Y0 spectroscopic standard. It is the only one of our Y dwarfs detected in all three short-wavelength bands of WISE ( $W 1$, $W 2$, and W3). Our spectrophotometric distance estimate places it at $10.5 \mathrm{pc}$; the available astrometry for this Y0 dwarf spans barely six months (Table 6), so we are not yet able to derive proper motion or parallax.

$$
\begin{gathered}
\text { A.23. WISEPA J174124.26+255319.5 }(\mathrm{J}=16.5 \mathrm{mag}, \\
W 2=12.3 \mathrm{mag})
\end{gathered}
$$

This nearby dwarf of near-infrared spectral type T9 is detected in the 2MASS and SDSS surveys but was overlooked because of its weak detection in both. Using a $10.4 \mathrm{yr}$ baseline, we find a high proper motion of $\mu=1.555 \pm 0.023$ arc- sec $\mathrm{yr}^{-1}$ and estimate a distance of $4.7 \mathrm{pc}$. Our preliminary trigonometric parallax measurement places it at $5.5_{-1.0}^{+1.4} \mathrm{pc}(\mathrm{Ta}-$ ble 7). This object has identical optical and near-infrared types to another nearby object, UGPS J072227.51-054031.2, whose trigonometric parallax from Lucas et al. (2010) places it at a distance of $4.1 \mathrm{pc}$. Gelino et al. (2011) note that WISE 1741+2553 appears single in near-infrared imaging observations with laser guide star adaptive optics.

$$
\begin{gathered}
\text { A.24. WISEPA J180435.40+311706.1 }(\mathrm{J}=18.7 \mathrm{mag}, \\
W 2=14.7 \mathrm{mag})
\end{gathered}
$$

The near-infrared spectrum of this object is classified as T9.5: because of its similarity to the spectrum of the T9.5 dwarf WISE 0148-7202. Although noisy, the narrowness of the $J$-band peaks falls intermediate between that of the T9 standard UGPS J072227.51-054031.2 and the Y0 standard WISE 1738+2732. 


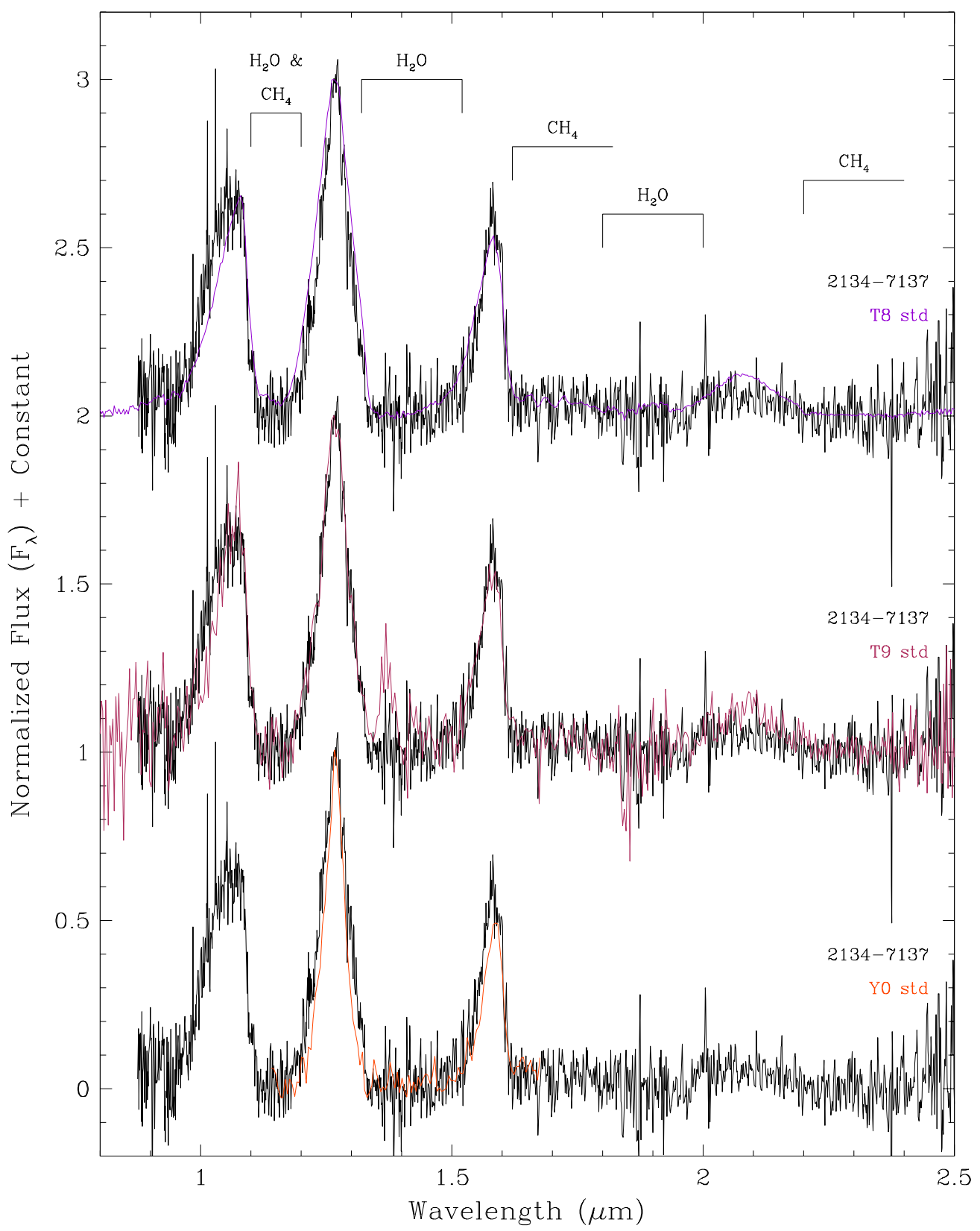

Figure 43. Near-infrared spectrum of WISE 2134-7137 (black) compared to the T8 (dark violet), T9 (maroon), and Y0 (orange red) spectral standards from Burgasser et al. (2006b) and Cushing et al. (2011). Spectra have been normalized to one at $1.28 \mu \mathrm{m}$ and integral offsets have been added to the $y$-axis values to separate the spectra vertically except where overplotting was intended. Prominent spectral features are marked.

(A color version of this figure is available in the online journal.)

We estimate that this object falls at a distance of $13.0 \mathrm{pc}$. The WISE detections for this object are very similar to those seen for Y dwarfs; namely, the object is detected only in bands $W 2$ and $W 3$ and not in $W 1$ or $W 4$ (Table 2).

\section{A.25. WISEPA J182831.08+265037.8 $(\mathrm{J}=23.6 \mathrm{mag}$, $W 2=14.3 \mathrm{mag})$}

This object, with a tentative classification of $>\mathrm{Y} 0$ from Cushing et al. (2011), is the latest object so far found with WISE. The spectrum is unique among late- $\mathrm{T}$ and $\mathrm{Y}$ dwarfs in that the $J$ - and $H$-band peaks, in units of $f_{\lambda}$, are nearly the same height (Figure 25). This reddening of the nearinfrared colors $(J-H=0.72 \pm 0.42 \mathrm{mag}$; Table 3$)$ is pre- dicted by model atmosphere calculations to occur at effective temperatures below 300-400 K (Burrows et al. 2003). This effect is due to the fact that the Wien tail of the spectral energy distribution becomes the overwhelming effect shaping the spectrum at those wavelengths, and this may be even more dramatically illustrated by the extremely red $J-W 2$ and $H-W 2$ colors measured for this object (Figures 7 and 8). Our spectrophotometric distance estimate of $<9.4 \mathrm{pc}$ implies exceedingly dim absolute magnitudes of $M_{J}>23.7$ and $M_{H}>23.0 \mathrm{mag}$ on the MKO filter system. These values agree with expectations that WISE $1828+2650$ should be dimmer at these wavelengths than the presumably warmer WISE 1541-2250, which also has exceedingly $\operatorname{dim} J$ and $H$ magnitudes (Table 7). 


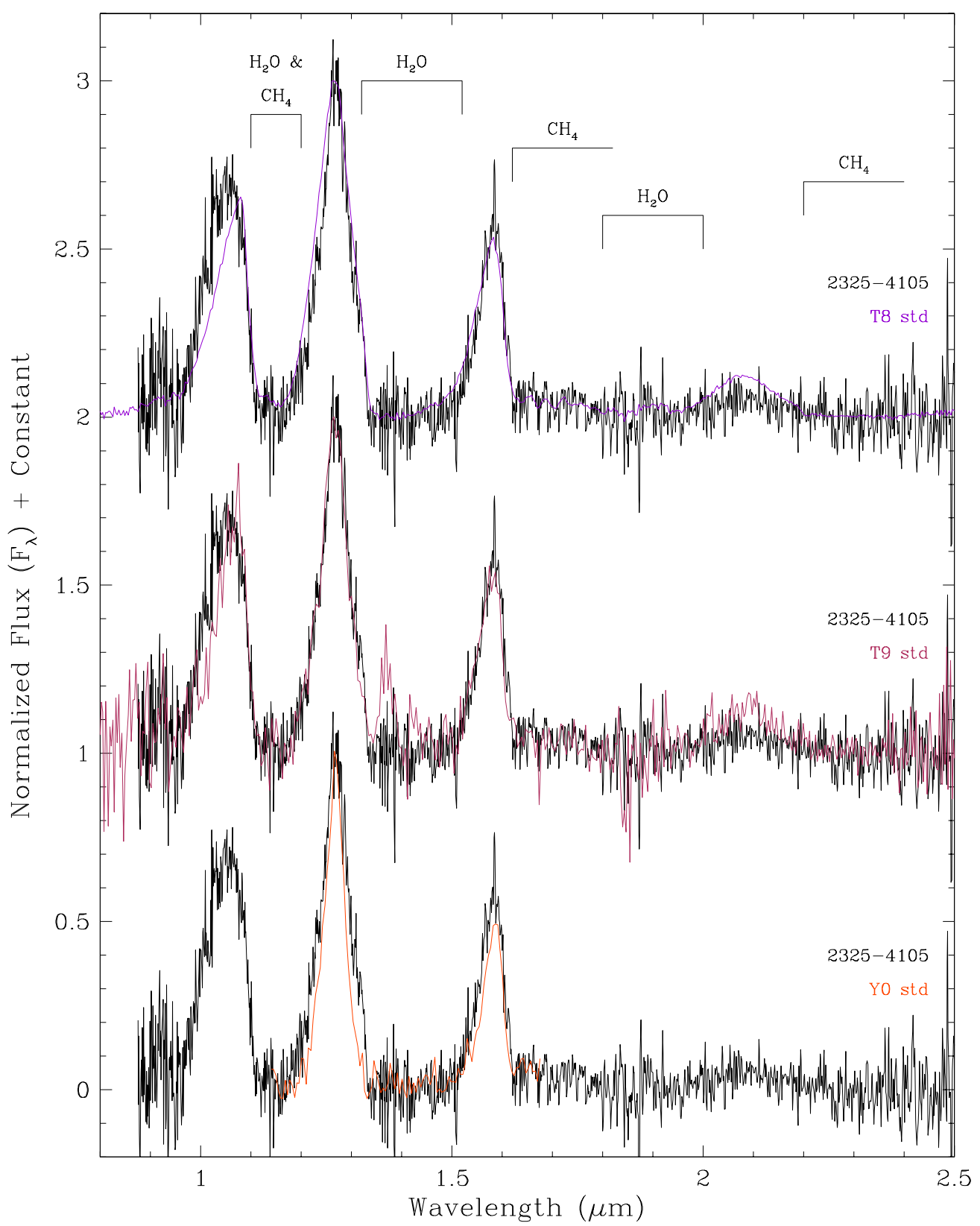

Figure 44. Near-infrared spectrum of WISE 2325-4105 (black) compared to the T8 (dark violet), T9 (maroon), and Y0 (orange red) spectral standards from Burgasser et al. (2006b) and Cushing et al. (2011). Spectra have been normalized to one at $1.28 \mu \mathrm{m}$ and integral offsets have been added to the $y$-axis values to separate the spectra vertically except where overplotting was intended. Prominent spectral features are marked.

(A color version of this figure is available in the online journal.)

\section{A.26. WISEPC J205628.90+145953.3 $(\mathrm{J}=19.2 \mathrm{mag}$, $W 2=13.9$ mag)}

This Y0 dwarf, discussed in Cushing et al. (2011), is estimated to lie at a distance of $7.7 \mathrm{pc}$. As with several other Y dwarf discoveries, this object is detected by WISE only in bands $W 2$ and $W 3$ and not in $W 1$ or $W 4$ (Table 2).

$$
\begin{gathered}
\text { A.27. WISEPA J213456.73-713743.6 }(J=19.8 \mathrm{mag}, \\
W 2=13.9 \mathrm{mag})
\end{gathered}
$$

The $J$-band spectrum of this object best fits the T9 standard, but there are discrepancies at the $Y$ and $K$ bands. At the $Y$ band the spectrum of WISE 2134-7137 shows excess flux relative to the standard, and at the $K$ band the spectrum shows less flux relative to the standard (Figure 43). We therefore classify this object as a "T9 pec." The physical cause, as discussed above for several other objects, may be low metal content.

$$
\begin{aligned}
& \text { A.28. WISEPC J232519.54-410534.9 }(J=19.7 \mathrm{mag}, \\
& W 2=14.1 \mathrm{mag})
\end{aligned}
$$

As with the previous object, the $J$-band spectrum best fits the T9 standard, but there are discrepancies at the $Y$ and $K$ bands. At the $Y$ band the spectrum of WISE 2325-4105 shows excess flux relative to the T9 standard, and at the $K$ band the spectrum shows less flux relative to the T9 standard (Figure 44). We therefore classify this object as a "T9 pec." The physical cause, as discussed above for other objects, may be low metal content. 


$$
\begin{gathered}
\text { A.29. WISEPC J232728.75-273056.5 }(J=16.7 \mathrm{mag}, \\
W 2=13.2 \mathrm{mag})
\end{gathered}
$$

This object has the near-infrared spectrum of a normal L9 dwarf, but its WISE color of $W 1-W 2=0.825 \pm 0.046$ is markedly redder than the other L9 dwarfs in Figure 1. The Spitzer/IRAC color of $\operatorname{ch} 1-\operatorname{ch} 2=0.247 \pm 0.025$ is redder than all other L and early-T dwarfs in Figure 11. Effects such as low-gravity or low-metallicity would cause the near-infrared spectrum of this object to appear unusually red or unusually blue, respectively, in the near-infrared (Kirkpatrick et al. 2010), and this is not seen. One hypothesis is that this object is an unresolved $\mathrm{L}+\mathrm{T}$ binary, but not one with such a warm $\mathrm{T}$ dwarf that the near-infrared spectrum of the composite shows itself to be peculiar. We can test this as follows. The $W 1-W 2$ color of WISE $2327-2730$ is $\sim 0.23$ mag redder than the mean $W 1-W 2$ for other L9 dwarfs. Using the absolute $W 2$ versus spectral type plot of Figure 29 along with the trend of $W 1-W 2$ color with spectral type in Figure 1, we estimate that the type of the hypothesized companion would have to be roughly T6.5. However, as Figure 4 of Burgasser (2007) shows, an object with a composite type of $\sim \mathrm{L} 9$ and secondary of $\sim \mathrm{T} 6.5$ would have a noticeably peculiar near-infrared spectrum which would distinguish it from a normal L9. Hence, binarity appears not to be the cause of the redder $W 1-W 2$ and $\operatorname{ch} 1-\operatorname{ch} 2$ colors. The reason for this color peculiarity remains unexplained.

Note added in proof: Scholz et al. (2011), whose work was refereed contemporaneously with this paper, independently discovered the T9 dwarf WISE $1741+2553$ first announced in Gelino et al. (2011) and also noted WISE 0254+0223 as a proper motion object of presumably late type.

\section{REFERENCES}

Albert, L., Artigau, É., Delorme, P., et al. 2011, AJ, 141, 203

Artigau, É., Doyon, R., Lafrenière, D., et al. 2006, ApJ, 651, L57

Artigau, É., Radigan, J., Folkes, S., et al. 2010, ApJ, 718, L38

Baraffe, I., Chabrier, G., Allard, F., \& Hauschildt, P. 2003, in IAU Symp. 211,

Brown Dwarfs, ed. E. Martín (San Francisco, CA: ASP), 41

Barman, T. S., Hauschildt, P. H., \& Allard, F. 2005, ApJ, 632, 1132

Bate, M. R., \& Bonnell, I. A. 2005, MNRAS, 356, 1201

Becklin, E. E., \& Zuckerman, B. 1988, Nature, 336, 656

Bertin, E., \& Arnouts, S. 1996, A\&AS, 117, 393

Bessell, M. S. 2005, ARA\&A, 43, 293

Bessell, M. S., \& Brett, J. M. 1988, PASP, 100, 1134

Bloom, J. S., Starr, D. L., Blake, C. H., Skrutskie, M. F., \& Falco, E. E. 2006, in ASP Conf. Ser. 351, Astronomical Data Analysis Software and Systems XV, ed. C. Gabriel, C. Arviset, D. Ponz, \& E. Solano (San Francisco, CA: ASP), 751

Bochanski, J. J., Burgasser, A. J., Simcoe, R. A., \& West, A. A. 2011, arXiv: 1109.2897

Boss, A. P. 2004, MNRAS, 350, L57

Bouvier, J., Kendall, T., \& Meeus, G. 2009, in ASP Conf. Proc. 1094, 15th Cambridge Workshop on Cool Stars, Stellar Systems and the Sun, ed. E. Stempels (San Francisco, CA: ASP), 497

Bouy, H., Brandner, W., Martín, E. L., et al. 2003, AJ, 126, 1526

Bowler, B. P., Liu, M. C., \& Dupuy, T. J. 2010, ApJ, 710, 45

Boyd, D. F. A., \& Whitworth, A. P. 2005, A\&A, 430, 1059

Burgasser, A. J. 2004, ApJS, 155, 191

Burgasser, A. J. 2007, AJ, 134, 1330

Burgasser, A. J. 2008, in ASP Conf. Ser. 384, 14th Cambridge Workshop on Cool Stars, Stellar Systems, and the Sun, ed. G. van Belle (San Francisco, CA: ASP), 126

Burgasser, A. J., Burrows, A., \& Kirkpatrick, J. D. 2006a, ApJ, 639, 1095

Burgasser, A. J., Cushing, M. C., Kirkpatrick, J. D., et al. 2011a, ApJ, 735, 116 Burgasser, A. J., Geballe, T. R., Leggett, S. K., Kirkpatrick, J. D., \& Golimowski, D. A. 2006 b, ApJ, 637, 1067

Burgasser, A. J., Kirkpatrick, J. D., Brown, M. E., et al. 1999, ApJ, 522, L65 Burgasser, A. J., Kirkpatrick, J. D., Brown, M. E., et al. 2002, ApJ, 564, 421 Burgasser, A. J., Kirkpatrick, J. D., Cutri, R. M., et al. 2000a, ApJ, 531, L57
Burgasser, A. J., Kirkpatrick, J. D., Liebert, J., \& Burrows, A. 2003a, ApJ, 594 510

Burgasser, A. J., Kirkpatrick, J. D., McElwain, M. W., et al. 2003b, AJ, 125, 850

Burgasser, A. J., Looper, D. L., Kirkpatrick, J. D., Cruz, K. L., \& Swift, B. J. 2008, ApJ, 674, 451

Burgasser, A. J., Looper, D., \& Rayner, J. T. 2010a, AJ, 139, 2448

Burgasser, A. J., McElwain, M. W., \& Kirkpatrick, J. D. 2003c, AJ, 126, 2487

Burgasser, A. J., McElwain, M. W., Kirkpatrick, J. D., et al. 2004, AJ, 127, 2856 Burgasser, A. J., Simcoe, R. A., Bochanski, J. J., et al. 2010b, ApJ, 725, 1405

Burgasser, A. J., Sitarski, B. N., Gelino, C. R., Logsdon, S. E., \& Perrin, M. D. 2011b, ApJ, 739, 49

Burgasser, A. J., Wilson, J. C., Kirkpatrick, J. D., et al. 2000b, AJ, 120, 1100

Burningham, B., Leggett, S. K., Homeier, D., et al. 2011b, MNRAS, 414, 3590

Burningham, B., Leggett, S. K., Lucas, P. W., et al. 2010a, MNRAS, 404, 1952 Burningham, B., Lucas, P. W., Leggett, S. K., et al. 2011a, MNRAS, 414, L90 Burningham, B., Pinfield, D. J., Leggett, S. K., et al. 2008, MNRAS, 391, 320 Burningham, B., Pinfield, D. J., Leggett, S. K., et al. 2009, MNRAS, 395, 1237 Burningham, B., Pinfield, D. J., Lucas, P. W., et al. 2010b, MNRAS, 406, 1885 Burrows, A., Sudarsky, D., \& Lunine, J. I. 2003, ApJ, 596, 587

Chiu, K., Fan, X., Leggett, S. K., et al. 2006, AJ, 131, 2722

Costa, E., Méndez, R. A., Jao, W.-C., et al. 2006, AJ, 132, 1234

Cruz, K. L., Burgasser, A. J., Reid, I. N., \& Liebert, J. 2004, ApJ, 604, L61

Cruz, K. L., Reid, I. N., Kirkpatrick, J. D., et al. 2007, AJ, 133, 439

Cruz, K. L., Reid, I. N., Liebert, J., Kirkpatrick, J. D., \& Lowrance, P. J. 2003, AJ, 126, 2421

Cushing, M. C., Kirkpatrick, J. D., Gelino, C. R., et al. 2011, ApJ, 743, 50

Cushing, M. C., Vacca, W. D., \& Rayner, J. T. 2004, PASP, 116, 362

Dahn, C. C., Harrington, R. S., Kallarakal, V. V., et al. 1988, AJ, 95, 237

Dahn, C. C., Harris, H. C., Vrba, F. J., et al. 2002, AJ, 124, 117

Deacon, N. R., Hambly, N. C., \& Cooke, J. A. 2005, A\&A, 435, 363

Deacon, N. R., Hambly, N. C., King, R. R., \& McCaughrean, M. J. 2009, MNRAS, 394, 857

Delfosse, X., Tinney, C. G., Forveille, T., et al. 1997, A\&A, 327, L25

Delfosse, X., Tinney, C. G., Forveille, T., et al. 1999, A\&AS, 135, 41

Delorme, P., Albert, L., Forveille, T., et al. 2010, A\&A, 518, A39

Delorme, P., Delfosse, X., Albert, L., et al. 2008, A\&A, 482, 961

Eddington, A. S. 1913, MNRAS, 73, 359

Eddington, A. S., Sir. 1940, MNRAS, 100, 354

Eisenhardt, P. R. M., Griffith, R. L., Stern, D., et al. 2010, AJ, 139, 2455

Ellis, S. C., Tinney, C. G., Burgasser, A. J., Kirkpatrick, J. D., \& McElwain, M. W. 2005, AJ, 130, 2347

Faherty, J. K., Burgasser, A. J., Cruz, K. L., et al. 2009, AJ, 137, 1

Fan, X., Knapp, G. R., Strauss, M. A., et al. 2000, AJ, 119, 928

Fazio, G. G., Hora, J. L., Allen, L. E., et al. 2004, ApJS, 154, 10

Fortney, J. J., Marley, M. S., Lodders, K., Saumon, D., \& Freedman, R. 2005, ApJ, 627, L69

Geballe, T. R., Knapp, G. R., Leggett, S. K., et al. 2002, ApJ, 564, 466

Gelino, C. R., Kirkpatrick, J. D., Cushing, M. C., et al. 2011, AJ, 142, 57

Giclas, H. L., Burnham, R., \& Thomas, N. G. 1971, Lowell Proper Motion Survey Northern Hemisphere: The G Numbered Stars (Flagstaff, AZ: Lowell Observatory)

Gizis, J. E. 2002, ApJ, 575, 484

Gizis, J. E., Monet, D. G., Reid, I. N., et al. 2000, AJ, 120, 1085

Goldman, B., Marsat, S., Henning, T., Clemens, C., \& Greiner, J. 2010, MNRAS, 405,1140

Hall, P. B. 2002, ApJ, 564, L89

Hamuy, M., Suntzeff, N. B., Heathcote, S. R., et al. 1994, PASP, 106, 566

Hanisch, R. J., Farris, A., Greisen, E. W., et al. 2001, A\&A, 376, 359

Hawkins, M. R. S., \& Bessell, M. S. 1988, MNRAS, 234, 177

Hawley, S. L., Covey, K. R., Knapp, G. R., et al. 2002, AJ, 123, 3409

Hayashi, C., \& Nakano, T. 1963, Prog. Theor. Phys., 30, 460

Herter, T. L., Henderson, C. P., Wilson, J. C., et al. 2008, Proc. SPIE, 7014 $70140 \mathrm{X}$

Horne, K. 1986, PASP, 98, 609

Kanneganti, S., Park, C., Skrutskie, M. F., et al. 2009, PASP, 121, 885

Kendall, T. R., Jones, H. R. A., Pinfield, D. J., et al. 2007, MNRAS, 374, 445

Kirkpatrick, J. D. 2000, in ASP Conf. Ser. 212, From Giant Planets to Cool Stars, ed. C. A. Griffith \& M. S. Marley (San Francisco, CA: ASP), 20

Kirkpatrick, J. D. 2005, ARA\&A, 43, 195

Kirkpatrick, J. D. 2008, in ASP Conf. Ser. 384, 14th Cambridge Workshop on Cool Stars, Stellar Systems, and the Sun, ed. G. van Belle (San Francisco, CA: ASP), 85

Kirkpatrick, J. D., Barman, T. S., Burgasser, A. J., et al. 2006, ApJ, 639, 1120 Kirkpatrick, J. D., Beichman, C. A., \& Skrutskie, M. F. 1997, ApJ, 476, 311 Kirkpatrick, J. D., Cruz, K. L., Barman, T. S., et al. 2008, ApJ, 689, 1295 Kirkpatrick, J. D., Dahn, C. C., Monet, D. G., et al. 2001, AJ, 121, 3235 
Kirkpatrick, J. D., Looper, D. L., Burgasser, A. J., et al. 2010, ApJS, 190, 100 Kirkpatrick, J. D., \& McCarthy, D. W., Jr. 1994, AJ, 107, 333

Kirkpatrick, J. D., McGraw, J. T., Hess, T. R., et al. 1994, ApJS, 94, 749

Kirkpatrick, J. D., Reid, I. N., Liebert, J., et al. 1999, ApJ, 519, 802

Kirkpatrick, J. D., Reid, I. N., Liebert, J., et al. 2000, AJ, 120, 447

Knapp, G. R., Leggett, S. K., Fan, X., et al. 2004, AJ, 127, 3553

Koekemoer, A. M., Fruchter, A. S., Hook, R. N., \& Hack, W. 2002, in Proc. Workshop held at the Space Telescope Science Institute, The 2002 HST Calibration Workshop: Hubble After the Installation of the ACS and the NICMOS Cooling System, ed. S. Arribas, A. Koekemoer, \& B. Whitmore (Baltimore, MD: STScI), 337

Kouzuma, S., \& Yamaoka, H. 2010, A\&A, 509, A64

Kumar, S. S. 1963, ApJ, 137, 1121

Kümmel, M., Walsh, J. R., Pirzkal, N., Kuntschner, H., \& Pasquali, A. 2009, PASP, 121, 59

Lawrence, A., Warren, S. J., Almaini, O., et al. 2007, MNRAS, 379, 1599

Leggett, S. K., Burningham, B., Saumon, D., et al. 2010, ApJ, 710, 1627

Leggett, S. K., Cushing, M. C., Saumon, D., et al. 2009, ApJ, 695, 1517

Leggett, S. K., Geballe, T. R., Fan, X., et al. 2000, ApJ, 536, L35

Lépine, S., \& Shara, M. M. 2005, AJ, 129, 1483

Liebert, J., Kirkpatrick, J. D., Cruz, K. L., et al. 2003, AJ, 125, 343

Liu, M. C., Delorme, P., Dupuy, T. J., et al. 2011, arXiv:1103.0014

Lodieu, N., Scholz, R.-D., \& McCaughrean, M. J. 2002, A\&A, 389, L20

Loh, E. D., Biel, J. D., Chen, J.-J., et al. 2004, Proc. SPIE, 5492, 1644

Looper, D. L., Kirkpatrick, J. D., \& Burgasser, A. J. 2007, AJ, 134, 1162

Looper, D. L., Kirkpatrick, J. D., Cutri, R. M., et al. 2008, ApJ, 686, 528

López-Morales, M. 2007, ApJ, 660, 732

Lucas, P. W., Tinney, C. G., Burningham, B., et al. 2010, MNRAS, 408, L56

Lucas, P. W., Weights, D. J., Roche, P. F., \& Riddick, F. C. 2006, MNRAS, 373, L60

Luhman, K. L. 2007, ApJS, 173, 104

Luhman, K. L., Burgasser, A. J., \& Bochanski, J. J. 2011, ApJ, 730, L9

Luhman, K. L., Patten, B. M., Marengo, M., et al. 2007, ApJ, 654, 570

Luhman, K. L., Rieke, G. H., Young, E. T., et al. 2000, ApJ, 540, 1016

Lutz, T. E., \& Kelker, D. H. 1973, PASP, 85, 573

Luyten, W. J. 1972, Proper Motion Survey with the Forty-eight Inch Schmidt Telescope. XXXI. Proper Motions for 2520 Faint Stars (Minneapolis, MN: Univ. Minnesota), 1

Luyten, W. J. 1974a, Proper Motion Survey with the Forty-eight Inch Schmidt Telescope._XXXVII. Proper Motions for 4483 Faint Stars (Minneapolis, MN: Univ. Minnesota), 1

Luyten, W. J. 1974b, Proper Motion Survey with the Forty-eight Inch Schmidt Telescope. XXXVI. Proper Motions for 6955 Faint Stars (Minneapolis, MN: Univ. Minnesota), 1

Luyten, W. J. 1979a, (2nd ed.; Minneapolis, MN: Univ. Minnesota)

Luyten, W. J. 1979b, NLTT Catalogue. Volume I. +90 degrees to +30 degrees (Minneapolis, MN: Univ. Minnesota), 1, 0

Luyten, W. J. 1979c, NLTT Catalogue. Volume II. +30 degrees to 0 degrees (Minneapolis, MN: Univ. Minnesota), 2, 0

Luyten, W. J. 1980, NLTT Catalogue. Volume III. 0 degrees to -30 degrees (Minneapolis, MN: Univ. Minnesota), 283

Luyten, W. J., \& Kowal, C. T. 1975, Proper Motion Survey with the Forty-eight Inch Schmidt Telescope. XLIII. One Hundred and Six Faint Stars with Large Proper Motions (Minneapolis, MN: Univ. Minnesota), 1

Mainzer, A., Cushing, M. C., Skrutskie, M., et al. 2011, ApJ, 726, 30

Malmquist, K. G. 1920, Medd. Lund Astron. Obs. Ser., 2., 22

Marocco, F., Smart, R. L., Jones, H. R. A., et al. 2010, A\&A, 524, A38

Marois, C., Macintosh, B., Barman, T., et al. 2008, Science, 322, 1348

Martín, E. L., Phan-Bao, N., Bessell, M., et al. 2010, A\&A, 517, A53

Martini, P., Persson, S. E., Murphy, D. C., et al. 2004, Proc. SPIE, 5492, 1653

McLean, I. S., Becklin, E. E., Bendiksen, O., et al. 1998, Proc. SPIE, 3354, 566

McLean, I. S., Graham, J. R., Becklin, E. E., et al. 2000, Proc. SPIE, 4008, 1048

McLean, I. S., Macintosh, B. A., Liu, T., et al. 1994, Proc. SPIE, 2198, 457

McLean, I. S., McGovern, M. R., Burgasser, A. J., et al. 2003, ApJ, 596, 561

Metchev, S. A., Kirkpatrick, J. D., Berriman, G. B., \& Looper, D. 2008, ApJ, 676,1281

Milligan, S., Cranton, B. W., \& Skrutskie, M. F. 1996, Proc. SPIE, 2863, 2

Monet, D. G., Levine, S. E., Canzian, B., et al. 2003, AJ, 125, 984

Muench, A. A., Lada, E. A., Lada, C. J., \& Alves, J. 2002, ApJ, 573, 366

Noll, K. S., Geballe, T. R., Leggett, S. K., \& Marley, M. S. 2000, ApJ, 541, L75

Oke, J. B., Cohen, J. G., Carr, M., et al. 1995, PASP, 107, 375
Padoan, P., Kritsuk, A., Michael, N. L., \& Nordlund, Å. 2005, Mem. Soc. Astron. Italiana, 76, 187

Patten, B. M., Stauffer, J. R., Burrows, A., et al. 2006, ApJ, 651, 502

Perryman, M. A. C., Lindegren, L., Kovalevsky, J., et al. 1997, A\&A, 323, L49

Phan-Bao, N., Bessell, M. S., Martín, E. L., et al. 2008, MNRAS, 383, 831

Pinfield, D. J., Burningham, B., Tamura, M., et al. 2008, MNRAS, 390, 304

Rayner, J. T., Toomey, D. W., Onaka, P. M., et al. 2003, PASP, 115, 362

Reid, I. N., Cruz, K. L., Kirkpatrick, J. D., et al. 2008, AJ, 136, 1290

Reid, I. N., Gizis, J. E., Kirkpatrick, J. D., \& Koerner, D. W. 2001, AJ, 121, 489

Reid, I. N., Kirkpatrick, J. D., Gizis, J. E., et al. 2000, AJ, 119, 369

Reid, I. N., Kirkpatrick, J. D., Liebert, J., et al. 2002, AJ, 124, 519

Reipurth, B., \& Clarke, C. 2001, AJ, 122, 432

Reylé, C., Delorme, P., Willott, C. J., et al. 2010, A\&A, 522, A112

Rodriguez, D. R., Zuckerman, B., Melis, C., \& Song, I. 2011, ApJ, 732, L29

Ross, F. E. 1928, AJ, 38, 117

Ruiz, M. T., Leggett, S. K., \& Allard, F. 1997, ApJ, 491, L107

Ruiz, M. T., Takamiya, M. Y., \& Roth, M. 1991, ApJ, 367, L59

Schilbach, E., Röser, S., \& Scholz, R.-D. 2009, A\&A, 493, L27

Schmidt, M. 1968, ApJ, 151, 393

Schmidt, S. J., West, A. A., Burgasser, A. J., Bochanski, J. J., \& Hawley, S. L. 2010, AJ, 139, 1045

Schneider, D. P., Knapp, G. R., Hawley, S. L., et al. 2002, AJ, 123, 458

Scholz, R.-D. 2010a, A\&A, 510, L8

Scholz, R.-D. 2010b, A\&A, 515, A92

Scholz, R.-D., Bihain, G., Schnurr, O., \& Storm, J. 2011, A\&A, 532, 5

Scholz, R.-D., McCaughrean, M. J., Lodieu, N., \& Kuhlbrodt, B. 2003, A\&A, 398, L29

Sheppard, S. S., \& Cushing, M. C. 2009, AJ, 137, 304

Simcoe, R. A., Burgasser, A. J., Bernstein, R. A., et al. 2008, Proc. SPIE, 7014, $70140 \mathrm{U}$

Simcoe, R. A., Burgasser, A. J., Bochanski, J. J., et al. 2010, Proc. SPIE, 7735, 773514

Skrutskie, M. F., Cutri, R. M., Stiening, R., et al. 2006, AJ, 131, 1163

Smith, J. D. T., Armus, L., Dale, D. A., et al. 2007, PASP, 119, 1133

Stephens, D. C., \& Leggett, S. K. 2004, PASP, 116, 9

Stern, D., Kirkpatrick, J. D., Allen, L. E., et al. 2007, ApJ, 663, 677

Strauss, M. A., Fan, X., Gunn, J. E., et al. 1999, ApJ, 522, L61

Sutherland, I. E., \& Hodgman, G. W. 1974, Commun., ACM, 17, 32

Swaters, R. A., Valdes, F., \& Dickinson, M. E. 2009, in ASP Conf. Ser. 411, Astronomical Data Analysis Software and Systems XVIII, ed. D. A. Bohlender, D. Durand, \& P. Dowler (San Francisco, CA: ASP), 506 Teerikorpi, P. 2004, A\&A, 424, 73

Tinney, C. G. 1993, AJ, 105, 1169

Tinney, C. G., Burgasser, A. J., \& Kirkpatrick, J. D. 2003, AJ, 126, 975

Tinney, C. G., Burgasser, A. J., Kirkpatrick, J. D., \& McElwain, M. W. 2005, AJ, 130,2326

Tody, D. 1986, Proc. SPIE, 627, 733

Tokunaga, A. T., Simons, D. A., \& Vacca, W. D. 2002, PASP, 114, 180

Tsvetanov, Z. I., Golimowski, D. A., Zheng, W., et al. 2000, ApJ, 531, L61

Vacca, W. D., Cushing, M. C., \& Rayner, J. T. 2003, PASP, 115, 389

van Altena, W. F., Lee, J. T., \& Hoffleit, D. 1995, VizieR Online Data Catalog, 1174,0

van Altena, W. F., Lee, J. T., \& Hoffleit, E. D. 2001, VizieR Online Data Catalog, 1238,0

van Dam, M. A., Bouchez, A. H., Le Mignant, D., et al. 2006, PASP, 118, 310 van Leeuwen, F. 2007, A\&A, 474, 653

Vrba, F. J., Henden, A. A., Luginbuhl, C. B., et al. 2004, AJ, 127, 2948

Warren, S. J., Mortlock, D. J., Leggett, S. K., et al. 2007, MNRAS, 381, 1400

Werner, M. W., Roellig, T. L., Low, F. J., et al. 2004, ApJS, 154, 1

West, A. A., Hawley, S. L., Bochanski, J. J., et al. 2008, AJ, 135, 785

Whitworth, A. P., \& Zinnecker, H. 2004, A\&A, 427, 299

Wilson, J. C., Eikenberry, S. S., Henderson, C. P., et al. 2003a, Proc. SPIE, 4841 , 451

Wilson, J. C., Kirkpatrick, J. D., Gizis, J. E., et al. 2001, AJ, 122, 1989

Wilson, J. C., Miller, N. A., Gizis, J. E., et al. 2003b, in IAU Symp. 211, Brown

Dwarfs, ed. E. Martín (San Francisco, CA: ASP), 197

Wizinowich, P. L., Le Mignant, D., Bouchez, A. H., et al. 2006, PASP, 118, 297

Wright, E. L., Eisenhardt, P. R. M., Mainzer, A. K., et al. 2010, AJ, 140, 1868

Wright, E. L., Mainzer, A., Gelino, C. R., \& Kirkpatrick, D. 2011, arXiv: 1104.2569

Wroblewski, H., \& Torres, C. 1991, A\&AS, 91, 129

York, D. G., Adelman, J., Anderson, J. E., Jr., et al. 2000, AJ, 120, 1579

Zhang, Z. H., Pokorny, R. S., Jones, H. R. A., et al. 2009, A\&A, 497, 619 